\title{
IUCrJ
}

Volume 6 (2019)

Supporting information for article:

Energetics of interactions in the solid state of 2-hydroxy-8-Xquinoline derivatives $(X=\mathrm{Cl}, \mathrm{Br}, \mathrm{I}, \mathrm{S}-\mathrm{Ph})$ : comparison of hirshfeld atom, $\mathrm{X}$-ray wavefunction and multipole refinements

Magdalena Woinska, Monika Wanat, Przemyslaw Taciak, Tomasz Pawinski, Wladek Minor and Krzysztof Wozniak 


\section{Energetics of Interactions in the Solid State on the Example of 2-Hydroxo-8-X-Quinoline Derivatives (X = Cl, Br, I, S-Ph): Comparison of Hirshfeld Atom, X-ray Wavefunction and Multipole Refinements}

\section{Statistical and structural parameters}

Table 1: Statistical parameters of the refinements (intensity cut-off: $|F| \geq 2 \sigma(|F|)$ ).

\begin{tabular}{|c|c|c|c|c|c|c|c|c|c|c|c|c|c|c|c|}
\hline & & \multicolumn{4}{|c|}{ PT-11(S-Ph) } & \multicolumn{4}{|c|}{ PT-2(Cl) } & \multicolumn{3}{|c|}{ PT-8(Br) } & \multicolumn{3}{|c|}{ PT-10(I) } \\
\hline & & HAR & XWR & $\mathrm{MM}$ & $\begin{array}{c}\text { MM } \\
\text { (freeXH) }\end{array}$ & HAR & XWR & $\mathrm{MM}$ & $\begin{array}{c}\text { MM } \\
\text { freeXH }\end{array}$ & HAR & XWR & $\mathrm{MM}$ & HAR & XWR & $\mathrm{MM}$ \\
\hline \multirow[t]{7}{*}{ harmonic } & $R$ & $1.61 \%$ & $1.50 \%$ & $1.47 \%$ & $1.46 \%$ & $2.93 \%$ & $2.70 \%$ & $2.71 \%$ & $2.71 \%$ & $2.50 \%$ & $2.43 \%$ & $2.51 \%$ & $2.08 \%$ & $1.80 \%$ & $1.91 \%$ \\
\hline & $w R$ & $2.73 \%$ & $2.59 \%$ & $2.57 \%$ & $2.56 \%$ & $3.22 \%$ & $3.02 \%$ & $3.02 \%$ & $3.02 \%$ & $2.64 \%$ & $2.50 \%$ & $2.42 \%$ & $2.65 \%$ & $2.25 \%$ & $2.48 \%$ \\
\hline & $G O F$ & 1.85 & 1.76 & 1.56 & 1.44 & 1.59 & 1.49 & 1.52 & 1.52 & 1.26 & 1.19 & 1.16 & 1.54 & 1.30 & 1.46 \\
\hline & $\rho_{\min }$ & -0.15 & -0.15 & -0.20 & -0.20 & -0.43 & -0.36 & -0.59 & -0.60 & -0.85 & -0.82 & -1.56 & -1.46 & -1.05 & -1.94 \\
\hline & $\rho_{\max }$ & 0.33 & 0.26 & 0.35 & 0.34 & 0.33 & 0.33 & 0.47 & 0.47 & 0.58 & 0.52 & 0.63 & 0.74 & 0.63 & 1.93 \\
\hline & $\chi^{2}$ & 3.43 & 3.88 & & & 2.52 & 2.22 & & & 1.58 & 1.42 & & 2.36 & 1.70 & \\
\hline & $\hat{\lambda}_{\max }$ & & 1.180 & & & & 1.570 & & & & 7.570 & & & 6.060 & \\
\hline \multirow{6}{*}{$\begin{array}{l}\text { anharmonic } \\
(\mathrm{n}=3)\end{array}$} & $R$ & $1.58 \%$ & & $1.44 \%$ & $1.44 \%$ & $2.92 \%$ & & $2.70 \%$ & $2.70 \%$ & $2.49 \%$ & & $2.50 \%$ & $1.90 \%$ & & $1.69 \%$ \\
\hline & $w R$ & $2.68 \%$ & & $2.51 \%$ & $2.51 \%$ & $3.19 \%$ & & $3.01 \%$ & $3.01 \%$ & $2.62 \%$ & & $2.40 \%$ & $2.45 \%$ & & $2.24 \%$ \\
\hline & $G O F$ & 1.82 & & 1.53 & 1.53 & 1.58 & & 1.51 & 1.51 & 1.25 & & 1.15 & 1.42 & & 1.32 \\
\hline & $\rho_{\min }$ & -0.14 & & -0.20 & -0.20 & -0.42 & & -0.48 & -0.48 & -0.80 & & -1.54 & -0.76 & & -1.05 \\
\hline & $\rho_{\max }$ & 0.18 & & 0.20 & 0.20 & 0.32 & & 0.47 & 0.47 & 0.58 & & 0.64 & 0.64 & & 1.16 \\
\hline & $\chi^{2}$ & 3.32 & & & & 2.49 & & & 1.56 & & & & 2.02 & & \\
\hline \multirow{7}{*}{$\begin{array}{l}\text { anharmonic } \\
(\mathrm{n}=3,4)\end{array}$} & $R$ & $1.58 \%$ & $1.48 \%$ & $1.44 \%$ & $1.44 \%$ & $2.60 \%$ & $2.51 \%$ & $2.66 \%$ & $2.67 \%$ & $2.43 \%$ & $2.36 \%$ & $2.43 \%$ & $1.80 \%$ & $1.70 \%$ & $1.68 \%$ \\
\hline & $w R$ & $2.67 \%$ & $2.56 \%$ & $2.51 \%$ & $2.51 \%$ & $3.01 \%$ & $2.88 \%$ & $2.98 \%$ & $2.98 \%$ & $2.56 \%$ & $2.42 \%$ & $2.34 \%$ & $2.40 \%$ & $2.18 \%$ & $2.22 \%$ \\
\hline & $G O F$ & 1.82 & 1.74 & 1.53 & 1.53 & 1.49 & 1.43 & 1.50 & 1.50 & 1.22 & 1.16 & 1.12 & 1.39 & 1.27 & 1.31 \\
\hline & $\rho_{\min }$ & -0.13 & -0.11 & -0.20 & -0.20 & -0.29 & -0.26 & -0.27 & -0.48 & -1.17 & -0.64 & -0.68 & -1.06 & -0.59 & -0.59 \\
\hline & $\rho_{\max }$ & 0.20 & 0.16 & 0.21 & 0.21 & 0.32 & 0.32 & 0.50 & 0.47 & 0.73 & 0.56 & 0.61 & 1.19 & 0.61 & 0.61 \\
\hline & $\chi^{2}$ & 3.29 & 3.02 & & & 2.21 & 2.03 & & & 1.50 & 1.34 & & 1.94 & 1.60 & \\
\hline & $\lambda_{\max }$ & & 1.200 & & & & 1.635 & & & & 7.750 & & & 6.015 & \\
\hline
\end{tabular}

Table 2: Similarity index for H ADPs between HAR and MM data (intensity cut-off: $|F| \geq 2 \sigma(|F|)$ ).

\begin{tabular}{llrrrr}
\hline \hline & Structure & PT-11(S-Ph) & PT-2 $(\mathrm{Cl})$ & PT-8(Br) & PT-10(I) \\
\hline harmonic & HAR/MM & 3.21 & 13.12 & $17.65^{*}$ & 3.42 \\
& HAR $_{\text {anis }} / \mathrm{MM}($ freeXH) & 3.18 & $\mathrm{n} / \mathrm{a}$ & $\mathrm{n} / \mathrm{a}$ & 3.45 \\
anharmonic & HAR/MM & 3.07 & 12.87 & $20.65^{* *}$ & 3.52 \\
$(\mathrm{n}=3)$ & HAR anis $/ M M($ freeXH) & 3.06 & $\mathrm{n} / \mathrm{a}$ & $\mathrm{n} / \mathrm{a}$ & 3.13 \\
anharmonic & HAR/MM & 2.22 & 14.38 & $29.16^{* *}$ & 3.39 \\
$(\mathrm{n}=3,4)$ & HAR/MM(freeXH) & 2.14 & $\mathrm{n} / \mathrm{a}$ & $\mathrm{n} / \mathrm{a}$ & 3.21 \\
\hline \hline
\end{tabular}

${ }^{*}$ The analysis excludes H2, H3, H8 and H1 for HAR refinement of PT-10(I) due to the presence of zero-positive values.

** The analysis excludes H2, H3, H8 for HAR refinement of PT-10(I) due to the presence of zero-positive values. 
Table 3: Bond lengths for PT-2(Cl) (intensity cut-off: $|F| \geq 2 \sigma(|F|)$ ).

\begin{tabular}{|c|c|c|c|c|c|c|c|c|c|c|}
\hline & opt & harm & $\begin{array}{c}\text { MM } \\
\text { anh, } n=3\end{array}$ & anh, $\mathrm{n}=3,4$ & harm & $\begin{array}{c}\mathrm{MM}(\text { freeXH }) \\
\text { anh, } n=3\end{array}$ & anh, $\mathrm{n}=3,4$ & harm & $\begin{array}{c}\text { HAR } \\
\text { anh, } n=3\end{array}$ & anh, $\mathrm{n}=3,4$ \\
\hline $\mathrm{C} 1-\mathrm{Cl} 1$ & 1.7536 & $1.7287(3)$ & $1.7284(3)$ & $1.7284(3)$ & $1.7287(3)$ & $1.7284(3)$ & $1.7284(3)$ & $1.7290(3)$ & $1.7282(3)$ & $1.7283(3)$ \\
\hline $\mathrm{C} 9-\mathrm{O} 1$ & 1.2439 & $1.2392(5)$ & $1.2392(5)$ & $1.2391(5)$ & $1.2392(5)$ & $1.2392(5)$ & $1.2391(5)$ & $1.2381(4)$ & $1.2381(4)$ & $1.2378(3)$ \\
\hline C6 - N1 & 1.3786 & $1.3762(4)$ & $1.3762(4)$ & $1.3763(4)$ & $1.3762(4)$ & $1.3762(4)$ & $1.3763(4)$ & $1.3765(4)$ & $1.3766(4)$ & $1.3766(3)$ \\
\hline C9 - N1 & 1.3829 & $1.3765(4)$ & $1.3765(4)$ & $1.3764(4)$ & $1.3765(4)$ & $1.3765(4)$ & $1.3764(4)$ & $1.3768(3)$ & $1.3768(3)$ & $1.3768(3)$ \\
\hline $\mathrm{C} 1-\mathrm{C} 2$ & 1.3863 & $1.3834(4)$ & $1.3834(4)$ & $1.3834(4)$ & $1.3834(4)$ & $1.3834(4)$ & $1.3834(4)$ & $1.3828(4)$ & $1.3829(4)$ & $1.3830(4)$ \\
\hline $\mathrm{C} 1-\mathrm{C} 6$ & 1.4127 & $1.4081(4)$ & $1.4081(4)$ & $1.4081(4)$ & $1.4081(4)$ & $1.4081(4)$ & $1.4081(4)$ & $1.4080(4)$ & $1.4080(4)$ & $1.4079(4)$ \\
\hline $\mathrm{C} 2-\mathrm{C} 3$ & 1.4036 & $1.4032(5)$ & $1.4032(5)$ & $1.4031(5)$ & $1.4032(5)$ & $1.4032(5)$ & $1.4031(5)$ & $1.4032(5)$ & $1.4032(5)$ & $1.4029(4)$ \\
\hline $\mathrm{C} 3-\mathrm{C} 4$ & 1.3854 & $1.3844(5)$ & $1.3844(5)$ & $1.3844(5)$ & $1.3844(5)$ & $1.3844(5)$ & $1.3844(5)$ & $1.3841(5)$ & $1.3841(5)$ & $1.3840(5)$ \\
\hline $\mathrm{C} 4-\mathrm{C} 5$ & 1.4107 & $1.4071(5)$ & $1.4072(4)$ & $1.4072(4)$ & $1.4072(5)$ & $1.4072(4)$ & $1.4072(4)$ & $1.4071(4)$ & $1.4071(4)$ & $1.4074(4)$ \\
\hline C5 - C6 & 1.4227 & $1.4138(4)$ & $1.4138(4)$ & $1.4137(4)$ & $1.4138(4)$ & $1.4138(4)$ & $1.4137(4)$ & $1.4133(4)$ & $1.4133(4)$ & $1.4131(4)$ \\
\hline $\mathrm{C} 5-\mathrm{C} 7$ & 1.4376 & $1.4368(4)$ & $1.4369(4)$ & $1.4369(4)$ & $1.4369(4)$ & $1.4369(4)$ & $1.4369(4)$ & $1.4369(4)$ & $1.4369(4)$ & $1.4371(4)$ \\
\hline $\mathrm{C} 7-\mathrm{C} 8$ & 1.3558 & $1.3533(5)$ & $1.3533(5)$ & $1.3534(5)$ & $1.3533(5)$ & $1.3533(5)$ & $1.3533(5)$ & $1.3526(4)$ & $1.3525(4)$ & $1.3525(4)$ \\
\hline C8 - C9 & 1.4541 & $1.4539(4)$ & $1.4538(4)$ & $1.4539(4)$ & $1.4538(4)$ & $1.4538(4)$ & $1.4538(4)$ & $1.4544(4)$ & $1.4543(4)$ & $1.4544(4)$ \\
\hline $\mathrm{C} 2-\mathrm{H} 2$ & 1.0870 & $1.09(1)$ & $1.09(1)$ & $1.09(1)$ & $1.12(3)$ & $1.12(3)$ & $1.12(3)$ & $1.105(7)$ & $1.105(7)$ & $1.096(7)$ \\
\hline $\mathrm{C} 3-\mathrm{H} 3$ & 1.0891 & $1.09(1)$ & $1.09(1)$ & $1.09(1)$ & $1.13(3)$ & $1.13(3)$ & $1.13(3)$ & $1.111(8)$ & $1.110(8)$ & $1.106(8)$ \\
\hline $\mathrm{C} 4-\mathrm{H} 4$ & 1.0915 & $1.08(1)$ & $1.08(1)$ & $1.08(1)$ & $1.08(3)$ & $1.08(3)$ & $1.08(3)$ & $1.082(8)$ & $1.080(8)$ & $1.078(7)$ \\
\hline $\mathrm{C} 7-\mathrm{H} 7$ & 1.0914 & $1.08(1)$ & $1.08(1)$ & $1.08(1)$ & $1.09(4)$ & $1.09(4)$ & $1.09(4)$ & $1.097(8)$ & $1.098(8)$ & $1.090(8)$ \\
\hline $\mathrm{C} 8-\mathrm{H} 8$ & 1.0870 & $1.10(1)$ & $1.10(1)$ & $1.10(1)$ & $1.17(3)$ & $1.16(3)$ & $1.16(3)$ & $1.099(8)$ & $1.097(8)$ & $1.088(8)$ \\
\hline $\mathrm{N} 1-\mathrm{H} 1$ & 1.0359 & $1.03(1)$ & $1.03(1)$ & $1.03(1)$ & $1.05(3)$ & $1.06(3)$ & $1.06(3)$ & $1.040(8)$ & $1.042(8)$ & $1.035(8)$ \\
\hline
\end{tabular}

Table 4: Bond lengths for PT-2(Cl) (no intensity cut-off).

\begin{tabular}{|c|c|c|c|c|c|c|c|c|c|c|}
\hline & opt & harm & $\begin{array}{c}\mathrm{MM} \\
\text { anh, } \mathrm{n}=3\end{array}$ & anh, $\mathrm{n}=3,4$ & harm & $\begin{array}{l}\mathrm{MM}(\text { freeXH }) \\
\text { anh, } \mathrm{n}=3\end{array}$ & anh, $\mathrm{n}=3,4$ & harm & $\begin{array}{c}\text { HAR } \\
\text { anh, } n=3\end{array}$ & anh, $\mathrm{n}=3,4$ \\
\hline $\mathrm{C} 1-\mathrm{Cl} 1$ & 1.7536 & $1.7289(3)$ & $1.7286(3)$ & $1.7287(3)$ & $1.7289(3)$ & $1.7286(3)$ & $1.7286(3)$ & $1.7290(3)$ & $1.7283(3)$ & $1.7283(3)$ \\
\hline C9 - O1 & 1.2439 & $1.2392(4)$ & $1.2392(4)$ & $1.2391(4)$ & $1.2392(4)$ & $1.2392(4)$ & $1.2391(4)$ & $1.2380(3)$ & $1.2380(3)$ & $1.2378(3)$ \\
\hline C6 - N1 & 1.3786 & $1.3761(4)$ & $1.3761(4)$ & $1.3762(4)$ & $1.3761(4)$ & $1.3761(4)$ & $1.3761(4)$ & $1.3765(3)$ & $1.3765(3)$ & $1.3765(3)$ \\
\hline C9 - N1 & 1.3829 & $1.3763(4)$ & $1.3764(4)$ & $1.3763(4)$ & $1.3764(4)$ & $1.3764(4)$ & $1.3763(4)$ & $1.3768(3)$ & $1.3768(3)$ & $1.3768(3)$ \\
\hline $\mathrm{C} 1-\mathrm{C} 2$ & 1.3863 & $1.3833(4)$ & $1.3833(4)$ & $1.3833(4)$ & $1.3833(4)$ & $1.3833(4)$ & $1.3834(4)$ & $1.3828(4)$ & $1.3829(4)$ & $1.3830(3)$ \\
\hline $\mathrm{C} 1-\mathrm{C} 6$ & 1.4127 & $1.4081(4)$ & $1.4081(4)$ & $1.4081(4)$ & $1.4080(4)$ & $1.4081(4)$ & $1.4080(4)$ & $1.4080(3)$ & $1.4080(3)$ & $1.4079(3)$ \\
\hline $\mathrm{C} 2-\mathrm{C} 3$ & 1.4036 & $1.4032(5)$ & $1.4032(5)$ & $1.4032(5)$ & $1.4033(5)$ & $1.4033(5)$ & $1.4032(5)$ & $1.4033(4)$ & $1.4033(4)$ & $1.4029(4)$ \\
\hline $\mathrm{C} 3-\mathrm{C} 4$ & 1.3854 & $1.3845(5)$ & $1.3845(5)$ & $1.3846(5)$ & $1.3844(5)$ & $1.3844(5)$ & $1.3845(5)$ & $1.3841(4)$ & $1.3841(4)$ & $1.3840(4)$ \\
\hline $\mathrm{C} 4-\mathrm{C} 5$ & 1.4107 & $1.4072(4)$ & $1.4072(4)$ & $1.4073(4)$ & $1.4072(4)$ & $1.4072(4)$ & $1.4073(4)$ & $1.4071(4)$ & $1.4071(4)$ & $1.4073(3)$ \\
\hline C5 - C6 & 1.4227 & $1.4138(4)$ & $1.4138(4)$ & $1.4137(4)$ & $1.4139(4)$ & $1.4138(4)$ & $1.4138(4)$ & $1.4133(3)$ & $1.4133(3)$ & $1.4132(3)$ \\
\hline $\mathrm{C} 5-\mathrm{C} 7$ & 1.4376 & $1.4366(4)$ & $1.4366(4)$ & $1.4367(4)$ & $1.4367(4)$ & $1.4366(4)$ & $1.4367(4)$ & $1.4369(4)$ & $1.4369(4)$ & $1.4371(3)$ \\
\hline $\mathrm{C} 7-\mathrm{C} 8$ & 1.3558 & $1.3536(4)$ & $1.3536(4)$ & $1.3536(4)$ & $1.3535(4)$ & $1.3535(4)$ & $1.3536(4)$ & $1.3526(4)$ & $1.3526(4)$ & $1.3525(4)$ \\
\hline C8 - C9 & 1.4541 & $1.4537(4)$ & $1.4537(4)$ & $1.4537(4)$ & $1.4536(4)$ & $1.4536(4)$ & $1.4537(4)$ & $1.4544(3)$ & $1.4543(3)$ & $1.4544(3)$ \\
\hline $\mathrm{C} 2-\mathrm{H} 2$ & 1.0870 & $1.084(8)$ & $1.084(8)$ & $1.084(8)$ & $1.12(2)$ & $1.12(2)$ & $1.12(2)$ & $1.106(7)$ & $1.106(7)$ & $1.096(6)$ \\
\hline C3 - H3 & 1.0891 & $1.083(8)$ & $1.083(8)$ & $1.083(8)$ & $1.12(2)$ & $1.12(2)$ & $1.12(2)$ & $1.111(7)$ & $1.110(7)$ & $1.105(7)$ \\
\hline $\mathrm{C} 4-\mathrm{H} 4$ & 1.0915 & $1.083(7)$ & $1.083(7)$ & $1.083(7)$ & $1.08(3)$ & $1.09(3)$ & $1.09(3)$ & $1.082(7)$ & $1.080(7)$ & $1.078(7)$ \\
\hline $\mathrm{C} 7-\mathrm{H} 7$ & 1.0914 & $1.084(7)$ & $1.084(7)$ & $1.083(7)$ & $1.08(3)$ & $1.08(3)$ & $1.08(3)$ & $1.096(7)$ & $1.097(7)$ & $1.091(7)$ \\
\hline $\mathrm{C} 8-\mathrm{H} 8$ & 1.0870 & $1.083(9)$ & $1.083(9)$ & $1.083(9)$ & $1.17(2)$ & $1.17(2)$ & $1.16(2)$ & $1.099(7)$ & $1.097(7)$ & $1.088(7)$ \\
\hline $\mathrm{N} 1$ - H1 & 1.0359 & $1.030(6)$ & $1.030(6)$ & $1.030(6)$ & $1.04(3)$ & $1.04(3)$ & $1.04(3)$ & $1.039(8)$ & $1.041(8)$ & $1.035(7)$ \\
\hline
\end{tabular}

Table 5: Bond lengths for PT-8(Br) (intensity cut-off: $|F| \geq 2 \sigma(|F|)$ ).

\begin{tabular}{|c|c|c|c|c|c|c|c|}
\hline & opt & harm & $\begin{array}{c}\mathrm{MM} \\
\text { anh, } \mathrm{n}=3\end{array}$ & anh, $\mathrm{n}=3,4$ & harm & $\begin{array}{c}\text { HAR } \\
\text { anh, n=3 }\end{array}$ & anh, $\mathrm{n}=3,4$ \\
\hline $\mathrm{C} 1-\mathrm{Br} 1$ & 1.8713 & $1.8834(6)$ & $1.8832(6)$ & $1.8832(6)$ & $1.8836(5)$ & $1.8834(5)$ & $1.8834(5)$ \\
\hline C9 - O1 & 1.2422 & $1.2359(9)$ & $1.2358(9)$ & $1.2357(9)$ & $1.2362(7)$ & $1.2361(7)$ & $1.2361(7)$ \\
\hline C6 - N1 & 1.3837 & $1.3776(7)$ & $1.3780(8)$ & $1.3781(8)$ & $1.3784(7)$ & $1.3784(7)$ & $1.3784(7)$ \\
\hline $\mathrm{C} 9-\mathrm{N} 1$ & 1.3824 & $1.3780(8)$ & $1.3776(7)$ & $1.3776(7)$ & $1.3769(7)$ & $1.3769(7)$ & $1.3769(6)$ \\
\hline $\mathrm{C} 1-\mathrm{C} 2$ & 1.3785 & $1.3843(8)$ & $1.3844(8)$ & $1.3843(8)$ & $1.3841(8)$ & $1.3841(8)$ & $1.3841(8)$ \\
\hline $\mathrm{C} 1$ - C6 & 1.3987 & $1.4078(8)$ & $1.4077(8)$ & $1.4077(7)$ & $1.4074(7)$ & $1.4074(7)$ & $1.4074(7)$ \\
\hline $\mathrm{C} 2-\mathrm{C} 3$ & 1.4002 & $1.4012(9)$ & $1.4012(9)$ & $1.4012(9)$ & $1.4010(9)$ & $1.4010(9)$ & $1.4009(9)$ \\
\hline $\mathrm{C} 3-\mathrm{C} 4$ & 1.3842 & $1.3827(10)$ & $1.3827(10)$ & $1.3827(9)$ & $1.3833(9)$ & $1.3833(9)$ & $1.3834(9)$ \\
\hline $\mathrm{C} 4-\mathrm{C} 5$ & 1.4086 & $1.4074(8)$ & $1.4075(8)$ & $1.4077(8)$ & $1.4068(8)$ & $1.4068(8)$ & $1.4069(8)$ \\
\hline C5 - C6 & 1.4212 & $1.4142(7)$ & $1.4142(7)$ & $1.4142(7)$ & $1.4140(7)$ & $1.4141(7)$ & $1.4140(7)$ \\
\hline C5 - C7 & 1.4372 & $1.4361(8)$ & $1.4361(8)$ & $1.4361(8)$ & $1.4364(8)$ & $1.4364(8)$ & $1.4365(8)$ \\
\hline $\mathrm{C} 7-\mathrm{C} 8$ & 1.3550 & $1.3517(9)$ & $1.3516(9)$ & $1.3517(9)$ & $1.3514(8)$ & $1.3514(8)$ & $1.3514(8)$ \\
\hline $\mathrm{C} 8$ - C9 & 1.4549 & $1.4544(8)$ & $1.4545(8)$ & $1.4544(7)$ & $1.4548(8)$ & $1.4548(7)$ & $1.4547(7)$ \\
\hline $\mathrm{C} 2-\mathrm{H} 2$ & 1.0868 & $1.083(1)$ & $1.083(1)$ & $1.083(1)$ & $1.08(1)$ & $1.08(1)$ & $1.08(1)$ \\
\hline C3 - H3 & 1.0891 & $1.083(1)$ & $1.083(1)$ & $1.083(1)$ & $1.10(1)$ & $1.10(1)$ & $1.10(1)$ \\
\hline $\mathrm{C} 4-\mathrm{H} 4$ & 1.0910 & $1.083(1)$ & $1.083(1)$ & $1.083(1)$ & $1.05(1)$ & $1.05(1)$ & $1.05(1)$ \\
\hline $\mathrm{C} 7-\mathrm{H} 7$ & 1.0909 & $1.083(1)$ & $1.083(1)$ & $1.083(1)$ & $1.08(2)$ & $1.08(2)$ & $1.08(1)$ \\
\hline $\mathrm{C} 8-\mathrm{H} 8$ & 1.0878 & $1.083(1)$ & $1.083(1)$ & $1.083(1)$ & $1.05(2)$ & $1.05(2)$ & $1.05(2)$ \\
\hline N1 - H1 & 1.0338 & $1.030(1)$ & $1.030(1)$ & $1.030(1)$ & $1.02(1)$ & $1.02(1)$ & $1.02(1)$ \\
\hline
\end{tabular}


Table 6: Bond lengths for PT-8(Br) (no intensity cut-off).

\begin{tabular}{|c|c|c|c|c|c|c|c|}
\hline & opt & harm & $\begin{array}{c}\mathrm{MM} \\
\text { anh, } \mathrm{n}=3\end{array}$ & anh, $\mathrm{n}=3,4$ & harm & $\begin{array}{c}\text { HAR } \\
\text { anh, } n=3\end{array}$ & anh, $\mathrm{n}=3,4$ \\
\hline $\mathrm{C} 1-\mathrm{Br} 1$ & 1.8713 & $1.8836(6)$ & $1.8834(6)$ & $1.8834(6)$ & $1.8836(5)$ & $1.8834(5)$ & $1.8834(5)$ \\
\hline C9 - O1 & 1.2422 & $1.2352(9)$ & $1.2351(9)$ & $1.2351(9)$ & $1.2361(6)$ & $1.2360(6)$ & $1.2360(6)$ \\
\hline C6 - N1 & 1.3837 & $1.3783(8)$ & $1.3783(8)$ & $1.3785(8)$ & $1.3784(6)$ & $1.3784(6)$ & $1.3784(6)$ \\
\hline C9 - N1 & 1.3824 & $1.3777(7)$ & $1.3777(7)$ & $1.3777(7)$ & $1.3769(6)$ & $1.3769(6)$ & $1.3769(6)$ \\
\hline $\mathrm{C} 1-\mathrm{C} 2$ & 1.3785 & $1.3838(8)$ & $1.3839(8)$ & $1.3837(8)$ & $1.3841(7)$ & $1.3841(7)$ & $1.3841(7)$ \\
\hline $\mathrm{C} 1$ - C6 & 1.3987 & $1.4078(8)$ & $1.4078(8)$ & $1.4077(7)$ & $1.4074(6)$ & $1.4073(6)$ & $1.4073(6)$ \\
\hline $\mathrm{C} 2-\mathrm{C} 3$ & 1.4002 & $1.4014(9)$ & $1.4014(9)$ & $1.4014(9)$ & $1.4009(8)$ & $1.4009(8)$ & $1.4008(8)$ \\
\hline C3 - C4 & 1.3842 & $1.383(1)$ & $1.383(1)$ & $1.3827(9)$ & $1.3833(8)$ & $1.3833(8)$ & $1.3833(8)$ \\
\hline $\mathrm{C} 4-\mathrm{C} 5$ & 1.4086 & $1.4076(8)$ & $1.4077(8)$ & $1.4078(8)$ & $1.4068(7)$ & $1.4069(7)$ & $1.4069(7)$ \\
\hline $\mathrm{C} 5-\mathrm{C} 6$ & 1.4212 & $1.4140(7)$ & $1.4141(7)$ & $1.4141(7)$ & $1.4140(6)$ & $1.4140(6)$ & $1.4140(6)$ \\
\hline $\mathrm{C} 5-\mathrm{C} 7$ & 1.4372 & $1.4358(8)$ & $1.4358(8)$ & $1.4359(8)$ & $1.4363(7)$ & $1.4363(7)$ & $1.4364(7)$ \\
\hline $\mathrm{C} 7-\mathrm{C} 8$ & 1.3550 & $1.3517(9)$ & $1.3517(9)$ & $1.3517(9)$ & $1.3515(7)$ & $1.3515(7)$ & $1.3514(7)$ \\
\hline C8 - C9 & 1.4549 & $1.4547(8)$ & $1.4547(8)$ & $1.4546(7)$ & $1.4548(7)$ & $1.4549(7)$ & $1.4548(7)$ \\
\hline $\mathrm{C} 2-\mathrm{H} 2$ & 1.0868 & $1.083(9)$ & $1.084(6)$ & $1.083(8)$ & $1.08(1)$ & $1.08(1)$ & $1.08(1)$ \\
\hline C3 - H3 & 1.0891 & $1.083(7)$ & $1.083(7)$ & $1.083(7)$ & $1.10(1)$ & $1.10(1)$ & $1.10(1)$ \\
\hline $\mathrm{C} 4-\mathrm{H} 4$ & 1.0910 & $1.084(6)$ & $1.084(6)$ & $1.083(6)$ & $1.05(1)$ & $1.05(1)$ & $1.05(1)$ \\
\hline $\mathrm{C} 7-\mathrm{H} 7$ & 1.0909 & $1.083(6)$ & $1.083(6)$ & $1.083(6)$ & $1.08(1)$ & $1.08(1)$ & $1.08(1)$ \\
\hline C8 - H8 & 1.0878 & $1.08(1)$ & $1.08(1)$ & $1.082(9)$ & $1.05(1)$ & $1.05(1)$ & $1.05(1)$ \\
\hline N1 - H1 & 1.0338 & $1.030(6)$ & $1.031(6)$ & $1.030(6)$ & $1.02(1)$ & $1.02(1)$ & $1.02(1)$ \\
\hline
\end{tabular}

Table 7: Bond lengths for PT-10(I) (intensity cut-off: $|F| \geq 2 \sigma(|F|)$ ).

\begin{tabular}{|c|c|c|c|c|c|c|c|}
\hline & opt & harm & $\begin{array}{c}\mathrm{MM} \\
\text { anh, } \mathrm{n}=3\end{array}$ & anh, $\mathrm{n}=3,4$ & harm & $\begin{array}{c}\text { HAR } \\
\text { anh, } n=3\end{array}$ & anh, $\mathrm{n}=3,4$ \\
\hline C1 - I1 & 2.1138 & $2.0872(9)$ & $2.0845(8)$ & $2.0843(8)$ & $2.0881(8)$ & $2.0857(7)$ & $2.0858(7)$ \\
\hline C9 - O1 & 1.2424 & $1.236(2)$ & $1.236(2)$ & $1.236(2)$ & 1.231(1) & $1.232(1)$ & 1.231(1) \\
\hline C6 - N1 & 1.3784 & $1.376(1)$ & $1.376(1)$ & $1.376(1)$ & $1.376(1)$ & $1.376(1)$ & $1.376(1)$ \\
\hline C9 - N1 & 1.3835 & $1.373(1)$ & $1.373(1)$ & $1.373(1)$ & $1.374(1)$ & $1.374(1)$ & $1.374(1)$ \\
\hline $\mathrm{C} 1-\mathrm{C} 2$ & 1.3892 & $1.387(1)$ & $1.387(1)$ & $1.387(1)$ & $1.385(1)$ & $1.385(1)$ & $1.385(1)$ \\
\hline $\mathrm{C} 1-\mathrm{C} 6$ & 1.4121 & $1.404(1)$ & $1.404(1)$ & $1.404(1)$ & $1.405(1)$ & $1.405(1)$ & $1.405(1)$ \\
\hline $\mathrm{C} 2-\mathrm{C} 3$ & 1.4043 & $1.403(2)$ & $1.403(1)$ & $1.403(1)$ & $1.401(1)$ & $1.401(1)$ & $1.401(1)$ \\
\hline $\mathrm{C} 3-\mathrm{C} 4$ & 1.3863 & $1.380(2)$ & $1.380(2)$ & $1.380(2)$ & $1.377(2)$ & $1.378(1)$ & $1.379(1)$ \\
\hline $\mathrm{C} 4-\mathrm{C} 5$ & 1.4097 & $1.404(2)$ & $1.404(1)$ & $1.404(1)$ & $1.404(1)$ & $1.404(1)$ & $1.404(1)$ \\
\hline $\mathrm{C} 5-\mathrm{C} 6$ & 1.4192 & $1.411(1)$ & $1.411(1)$ & $1.411(1)$ & $1.410(1)$ & $1.409(1)$ & $1.409(1)$ \\
\hline C5 - C7 & 1.4401 & $1.435(2)$ & $1.435(1)$ & $1.435(1)$ & $1.435(1)$ & $1.434(1)$ & $1.435(1)$ \\
\hline $\mathrm{C} 7-\mathrm{C} 8$ & 1.3576 & $1.354(2)$ & $1.356(2)$ & $1.356(2)$ & $1.354(2)$ & $1.355(1)$ & $1.355(1)$ \\
\hline $\mathrm{C} 8-\mathrm{C} 9$ & 1.4547 & $1.451(1)$ & $1.450(1)$ & $1.450(1)$ & $1.450(1)$ & $1.451(1)$ & $1.451(1)$ \\
\hline $\mathrm{C} 2-\mathrm{H} 2$ & 1.0879 & $1.083(1)$ & $1.083(1)$ & $1.083(1)$ & $1.08(2)$ & $1.09(2)$ & $1.09(2)$ \\
\hline C3 - H3 & 1.0906 & $1.083(1)$ & $1.083(1)$ & $1.083(1)$ & $1.00(2)$ & $1.05(2)$ & $1.04(2)$ \\
\hline $\mathrm{C} 4-\mathrm{H} 4$ & 1.0921 & $1.083(1)$ & $1.083(1)$ & $1.083(1)$ & $1.09(2)$ & $1.08(2)$ & $1.08(2)$ \\
\hline $\mathrm{C} 7-\mathrm{H} 7$ & 1.0888 & $1.083(1)$ & $1.083(1)$ & $1.083(1)$ & $1.01(2)$ & $1.02(2)$ & $1.02(2)$ \\
\hline $\mathrm{C} 8-\mathrm{H} 8$ & 1.0895 & $1.083(1)$ & $1.083(1)$ & $1.083(1)$ & $1.05(2)$ & $1.07(2)$ & $1.07(2)$ \\
\hline N1 - H1 & 1.0167 & $1.030(1)$ & $1.030(1)$ & $1.030(1)$ & $0.92(2)$ & $0.89(2)$ & $0.88(2)$ \\
\hline
\end{tabular}

Table 8: Bond lengths for PT-10(I) (no intensity cut-off).

\begin{tabular}{|c|c|c|c|c|c|c|c|}
\hline & opt & harm & $\begin{array}{c}\mathrm{MM} \\
\text { anh, } \mathrm{n}=3\end{array}$ & anh, $\mathrm{n}=3,4$ & harm & $\begin{array}{c}\text { HAR } \\
\text { anh, } n=3\end{array}$ & anh, $\mathrm{n}=3,4$ \\
\hline C1 - I1 & 2.1138 & $2.0862(9)$ & $2.0833(8)$ & $2.0833(8)$ & $2.0881(8)$ & $2.0857(7)$ & $2.0858(7)$ \\
\hline C9 - O1 & 1.2424 & $1.235(2)$ & $1.236(2)$ & $1.236(2)$ & $1.231(1)$ & $1.232(1)$ & 1.231(1) \\
\hline C6 - N1 & 1.3784 & $1.377(1)$ & $1.377(1)$ & $1.377(1)$ & $1.376(1)$ & $1.3759(9)$ & $1.3757(9)$ \\
\hline C9 - N1 & 1.3835 & $1.374(1)$ & $1.374(1)$ & $1.374(1)$ & $1.374(1)$ & 1.374(1) & $1.3736(9)$ \\
\hline $\mathrm{C} 1-\mathrm{C} 2$ & 1.3892 & $1.387(1)$ & $1.388(1)$ & $1.388(1)$ & $1.385(1)$ & $1.385(1)$ & $1.385(1)$ \\
\hline $\mathrm{C} 1-\mathrm{C} 6$ & 1.4121 & $1.404(1)$ & $1.404(1)$ & $1.404(1)$ & $1.405(1)$ & $1.405(1)$ & $1.405(1)$ \\
\hline $\mathrm{C} 2-\mathrm{C} 3$ & 1.4043 & $1.403(2)$ & $1.402(1)$ & $1.402(1)$ & $1.402(1)$ & $1.401(1)$ & $1.401(1)$ \\
\hline $\mathrm{C} 3-\mathrm{C} 4$ & 1.3863 & $1.379(2)$ & $1.380(2)$ & $1.380(2)$ & $1.377(1)$ & $1.378(1)$ & $1.379(1)$ \\
\hline $\mathrm{C} 4-\mathrm{C} 5$ & 1.4097 & $1.405(2)$ & $1.405(1)$ & $1.405(1)$ & $1.404(1)$ & $1.404(1)$ & $1.404(1)$ \\
\hline C5 - C6 & 1.4192 & $1.411(1)$ & $1.411(1)$ & $1.411(1)$ & $1.409(1)$ & $1.409(1)$ & $1.4093(9)$ \\
\hline $\mathrm{C} 5-\mathrm{C} 7$ & 1.4401 & $1.436(2)$ & $1.435(1)$ & $1.435(1)$ & $1.435(1)$ & $1.434(1)$ & $1.434(1)$ \\
\hline $\mathrm{C} 7-\mathrm{C} 8$ & 1.3576 & $1.353(2)$ & $1.354(2)$ & $1.354(2)$ & $1.354(1)$ & $1.355(1)$ & $1.355(1)$ \\
\hline $\mathrm{C} 8-\mathrm{C} 9$ & 1.4547 & $1.452(1)$ & $1.452(1)$ & $1.451(1)$ & $1.450(1)$ & $1.451(1)$ & $1.451(1)$ \\
\hline $\mathrm{C} 2-\mathrm{H} 2$ & 1.0879 & $1.082(9)$ & $1.082(8)$ & $1.082(8)$ & $1.07(2)$ & $1.09(2)$ & $1.09(2)$ \\
\hline C3 - H3 & 1.0906 & $1.083(8)$ & $1.085(9)$ & $1.083(9)$ & $1.00(2)$ & $1.04(2)$ & $1.03(2)$ \\
\hline $\mathrm{C} 4-\mathrm{H} 4$ & 1.0921 & $1.08(1)$ & $1.08(1)$ & $1.08(1)$ & $1.09(2)$ & $1.08(2)$ & $1.08(2)$ \\
\hline $\mathrm{C} 7-\mathrm{H} 7$ & 1.0888 & $1.08(1)$ & $1.08(1)$ & $1.08(1)$ & $1.02(2)$ & $1.03(2)$ & $1.02(2)$ \\
\hline $\mathrm{C} 8-\mathrm{H} 8$ & 1.0895 & $1.08(1)$ & $1.08(1)$ & $1.08(1)$ & $1.05(2)$ & $1.07(2)$ & $1.07(2)$ \\
\hline N1 - H1 & 1.0167 & $1.03(1)$ & $1.03(1)$ & $1.03(1)$ & $0.92(2)$ & $0.89(2)$ & $0.88(2)$ \\
\hline
\end{tabular}


Table 9: Bond lengths for PT-11(S-Ph) (intensity cut-off: $|F| \geq 2 \sigma(|F|))$.

\begin{tabular}{|c|c|c|c|c|c|c|c|c|c|c|}
\hline & opt & harm & $\begin{array}{c}\mathrm{MM} \\
\text { anh, } \mathrm{n}=3\end{array}$ & anh, $\mathrm{n}=3,4$ & harm & $\begin{array}{c}\mathrm{MM}(\text { freeXH } \\
\text { anh, } \mathrm{n}=3\end{array}$ & anh, $\mathrm{n}=3,4$ & harm & $\begin{array}{c}\text { HAR } \\
\text { anh, } n=3\end{array}$ & anh, $\mathrm{n}=3,4$ \\
\hline C8 - S1 & 1.7895 & $1.7667(1)$ & $1.7661(2)$ & $1.7661(2)$ & $1.7667(1)$ & $1.7661(2)$ & $1.7661(2)$ & $1.7672(2)$ & $1.7665(2)$ & $1.7666(2)$ \\
\hline $\mathrm{C} 10-\mathrm{S} 1$ & 1.7980 & $1.7742(2)$ & $1.7728(2)$ & $1.7728(2)$ & $1.7742(2)$ & $1.7728(2)$ & $1.7728(2)$ & $1.7747(2)$ & $1.7733(2)$ & $1.7733(2)$ \\
\hline $\mathrm{C} 1$ - O1 & 1.2446 & $1.2390(2)$ & $1.2390(2)$ & $1.2390(2)$ & $1.2390(2)$ & $1.2390(2)$ & $1.2390(2)$ & $1.2382(2)$ & $1.2382(2)$ & $1.2382(2)$ \\
\hline $\mathrm{C} 1$ - N1 & 1.3844 & $1.3775(2)$ & $1.3776(2)$ & $1.3776(2)$ & $1.3775(2)$ & $1.3775(2)$ & $1.3776(2)$ & $1.3777(2)$ & $1.3777(2)$ & $1.3777(2)$ \\
\hline C9 - N1 & 1.3815 & $1.3818(2)$ & $1.3819(2)$ & $1.3818(2)$ & $1.3818(2)$ & $1.3818(2)$ & $1.3818(2)$ & $1.3821(2)$ & $1.3821(2)$ & $1.3821(2)$ \\
\hline $\mathrm{C} 1-\mathrm{C} 2$ & 1.4575 & $1.4585(2)$ & $1.4585(2)$ & $1.4585(2)$ & $1.4585(2)$ & $1.4585(2)$ & $1.4585(2)$ & $1.4586(2)$ & $1.4585(2)$ & $1.4586(2)$ \\
\hline $\mathrm{C} 2-\mathrm{C} 3$ & 1.3577 & $1.3563(3)$ & $1.3563(3)$ & $1.3563(3)$ & $1.3563(2)$ & $1.3563(3)$ & $1.3563(3)$ & $1.3557(3)$ & $1.3558(3)$ & $1.3558(3)$ \\
\hline C3 - C4 & 1.4381 & $1.4383(2)$ & $1.4382(2)$ & $1.4382(2)$ & $1.4382(2)$ & $1.4382(2)$ & $1.4382(2)$ & $1.4384(3)$ & $1.4384(3)$ & $1.4384(3)$ \\
\hline $\mathrm{C} 4-\mathrm{C} 5$ & 1.4091 & $1.4069(2)$ & $1.4069(2)$ & $1.4069(2)$ & $1.4069(2)$ & $1.4069(2)$ & $1.4069(2)$ & $1.4066(2)$ & $1.4066(2)$ & $1.4066(2)$ \\
\hline $\mathrm{C} 4-\mathrm{C} 9$ & 1.4230 & $1.4141(2)$ & $1.4142(2)$ & $1.4142(2)$ & $1.4141(2)$ & $1.4142(2)$ & $1.4142(2)$ & $1.4142(2)$ & $1.4142(2)$ & $1.4142(2)$ \\
\hline C5 - C6 & 1.3866 & $1.3870(3)$ & $1.3869(3)$ & $1.3870(3)$ & $1.3870(3)$ & $1.3869(3)$ & $1.3870(3)$ & $1.3860(3)$ & $1.3860(3)$ & $1.3860(3)$ \\
\hline C6 - C7 & 1.4017 & $1.4008(3)$ & $1.4009(2)$ & $1.4009(2)$ & $1.4008(2)$ & $1.4009(2)$ & $1.4009(2)$ & $1.4007(3)$ & $1.4008(3)$ & $1.4007(3)$ \\
\hline $\mathrm{C} 7-\mathrm{C} 8$ & 1.3933 & $1.3923(2)$ & $1.3922(2)$ & $1.3922(2)$ & $1.3923(2)$ & $1.3922(2)$ & $1.3922(2)$ & $1.3921(2)$ & $1.3920(2)$ & $1.3920(2)$ \\
\hline C8 - C9 & 1.4184 & $1.4147(2)$ & $1.4147(2)$ & $1.4147(2)$ & $1.4146(2)$ & $1.4147(2)$ & $1.4147(2)$ & $1.4141(2)$ & $1.4142(2)$ & $1.4142(2)$ \\
\hline $\mathrm{C} 10-\mathrm{C} 11$ & 1.4013 & $1.3978(2)$ & $1.3979(2)$ & $1.3979(2)$ & $1.3978(2)$ & $1.3979(2)$ & $1.3978(2)$ & $1.3977(2)$ & $1.3978(2)$ & $1.3978(2)$ \\
\hline $\mathrm{C} 10-\mathrm{C} 15$ & 1.4013 & $1.3997(2)$ & $1.3997(2)$ & $1.3997(2)$ & $1.3997(2)$ & $1.3997(2)$ & $1.3997(2)$ & $1.3990(2)$ & $1.3990(2)$ & $1.3990(2)$ \\
\hline $\mathrm{C} 11-\mathrm{C} 12$ & 1.3967 & $1.3951(3)$ & $1.3951(3)$ & $1.3951(3)$ & $1.3951(3)$ & $1.3951(3)$ & $1.3951(3)$ & $1.3951(3)$ & $1.3950(3)$ & $1.3950(3)$ \\
\hline C12 - C13 & 1.3983 & $1.3989(3)$ & $1.3988(3)$ & $1.3988(3)$ & $1.3989(3)$ & $1.3989(3)$ & $1.3989(3)$ & $1.3983(4)$ & $1.3982(3)$ & $1.3982(3)$ \\
\hline $\mathrm{C} 13-\mathrm{C} 14$ & 1.3983 & $1.3966(3)$ & $1.3966(3)$ & $1.3966(3)$ & $1.3966(3)$ & $1.3966(3)$ & $1.3967(3)$ & $1.3960(3)$ & $1.3962(3)$ & $1.3962(3)$ \\
\hline C14 - C15 & 1.3973 & $1.3948(3)$ & $1.3948(3)$ & $1.3948(3)$ & $1.3948(3)$ & $1.3949(3)$ & $1.3949(3)$ & $1.3947(3)$ & $1.3948(3)$ & $1.3947(3)$ \\
\hline $\mathrm{C} 2-\mathrm{H} 2$ & 1.0888 & $1.083(2)$ & $1.083(2)$ & $1.083(2)$ & $1.09(2)$ & $1.08(2)$ & $1.08(2)$ & $1.075(7)$ & $1.076(7)$ & $1.073(7)$ \\
\hline C3 - H3 & 1.0936 & $1.083(2)$ & $1.083(2)$ & $1.083(2)$ & $1.11(2)$ & $1.11(2)$ & $1.11(2)$ & $1.084(7)$ & $1.084(7)$ & $1.084(7)$ \\
\hline C5 - H5 & 1.0914 & $1.083(2)$ & $1.083(2)$ & $1.083(2)$ & $1.05(2)$ & $1.06(2)$ & $1.06(2)$ & $1.073(7)$ & $1.074(7)$ & $1.074(7)$ \\
\hline C6 - H6 & 1.0885 & $1.083(2)$ & $1.083(2)$ & $1.083(2)$ & $1.07(2)$ & $1.07(2)$ & $1.07(2)$ & $1.073(7)$ & $1.072(7)$ & $1.071(7)$ \\
\hline $\mathrm{C} 7-\mathrm{H} 7$ & 1.0897 & $1.083(2)$ & $1.083(2)$ & $1.083(2)$ & $1.10(2)$ & $1.11(2)$ & $1.11(2)$ & $1.080(7)$ & $1.083(7)$ & $1.081(7)$ \\
\hline C11 - H11 & 1.0912 & $1.083(2)$ & $1.083(2)$ & $1.083(2)$ & $1.14(2)$ & $1.14(2)$ & $1.14(2)$ & $1.109(7)$ & $1.109(7)$ & $1.108(7)$ \\
\hline $\mathrm{C} 12-\mathrm{H} 12$ & 1.0891 & $1.083(2)$ & $1.083(2)$ & $1.083(2)$ & $1.12(2)$ & $1.12(2)$ & $1.12(2)$ & $1.078(7)$ & $1.079(6)$ & $1.077(6)$ \\
\hline $\mathrm{C} 13-\mathrm{H} 13$ & 1.0898 & $1.083(2)$ & $1.083(2)$ & $1.083(2)$ & $1.10(2)$ & $1.10(2)$ & $1.10(2)$ & $1.068(7)$ & $1.069(7)$ & $1.068(7)$ \\
\hline C14 - H14 & 1.0903 & $1.083(2)$ & $1.083(2)$ & $1.083(2)$ & $1.11(2)$ & $1.12(2)$ & $1.12(2)$ & $1.090(8)$ & $1.090(8)$ & $1.089(8)$ \\
\hline C15 - H15 & 1.0871 & $1.083(2)$ & $1.083(2)$ & $1.083(2)$ & $1.05(2)$ & $1.05(2)$ & $1.05(2)$ & $1.081(6)$ & $1.082(6)$ & $1.081(6)$ \\
\hline N1 - H1 & 1.0311 & $1.030(2)$ & $1.030(2)$ & $1.030(2)$ & $0.98(2)$ & $0.99(2)$ & $0.98(2)$ & $1.041(6)$ & $1.042(6)$ & $1.042(6)$ \\
\hline
\end{tabular}

Table 10: Bond lengths for PT-11(S-Ph) (no intensity cut-off).

\begin{tabular}{|c|c|c|c|c|c|c|c|c|c|c|}
\hline & \multirow[b]{2}{*}{ opt } & \multirow{2}{*}{\multicolumn{2}{|c|}{$\begin{array}{c}\text { MM } \\
\text { anh, } n=3\end{array}$}} & \multirow[b]{2}{*}{ anh, $\mathrm{n}=3,4$} & \multicolumn{3}{|c|}{$\mathrm{MM}($ freeXH) } & \multicolumn{3}{|c|}{ HAR } \\
\hline & & & & & harm & anh, $\mathrm{n}=3$ & anh, $\mathrm{n}=3,4$ & harm & anh, $\mathrm{n}=3$ & anh, $\mathrm{n}=3,4$ \\
\hline $\mathrm{C} 8-\mathrm{S} 1$ & 1.7895 & $1.7667(2)$ & $1.7662(2)$ & $1.7662(2)$ & $1.7667(2)$ & $1.7662(2)$ & $1.7662(2)$ & $1.7672(1)$ & $1.7665(2)$ & $1.7666(2)$ \\
\hline C10 - S1 & 1.7980 & $1.7741(2)$ & $1.7729(2)$ & $1.7729(2)$ & $1.7742(2)$ & $1.7729(2)$ & $1.7729(2)$ & $1.7747(2)$ & $1.7733(2)$ & $1.7734(2)$ \\
\hline $\mathrm{C} 1-\mathrm{O} 1$ & 1.2446 & $1.2389(3)$ & $1.2389(3)$ & $1.2389(3)$ & $1.2389(3)$ & $1.2389(3)$ & $1.2389(3)$ & $1.2382(2)$ & $1.2382(2)$ & $1.2382(2)$ \\
\hline $\mathrm{C} 1-\mathrm{N} 1$ & 1.3844 & $1.3776(2)$ & $1.3777(2)$ & $1.3777(2)$ & $1.3776(2)$ & $1.3776(2)$ & $1.3777(2)$ & $1.3777(2)$ & $1.3777(2)$ & $1.3777(2)$ \\
\hline $\mathrm{C} 9-\mathrm{N} 1$ & 1.3815 & $1.3818(2)$ & $1.3818(2)$ & $1.3818(2)$ & $1.3818(2)$ & $1.3818(2)$ & $1.3817(2)$ & $1.3821(2)$ & $1.3821(2)$ & $1.3821(2)$ \\
\hline $\mathrm{C} 1-\mathrm{C} 2$ & 1.4575 & $1.4584(2)$ & $1.4583(2)$ & $1.4583(2)$ & $1.4584(2)$ & $1.4583(2)$ & $1.4583(2)$ & $1.4586(2)$ & $1.4585(2)$ & $1.4586(2)$ \\
\hline $\mathrm{C} 2-\mathrm{C} 3$ & 1.3577 & $1.3566(3)$ & $1.3566(3)$ & $1.3566(3)$ & $1.3566(3)$ & $1.3566(3)$ & $1.3566(3)$ & $1.3558(3)$ & $1.3558(3)$ & $1.3558(3)$ \\
\hline C3 - C4 & 1.4381 & $1.4383(3)$ & $1.4383(3)$ & $1.4383(3)$ & $1.4384(3)$ & $1.4383(3)$ & $1.4383(3)$ & $1.4384(2)$ & $1.4383(2)$ & $1.4384(2)$ \\
\hline $\mathrm{C} 4-\mathrm{C} 5$ & 1.4091 & $1.4068(3)$ & $1.4068(2)$ & $1.4068(2)$ & $1.4068(3)$ & $1.4068(2)$ & $1.4068(2)$ & $1.4066(2)$ & $1.4066(2)$ & $1.4066(2)$ \\
\hline C4 - C9 & 1.4230 & $1.4141(2)$ & $1.4142(2)$ & $1.4142(2)$ & $1.4141(2)$ & $1.4142(2)$ & $1.4142(2)$ & $1.4142(2)$ & $1.4142(2)$ & $1.4142(2)$ \\
\hline C5 - C6 & 1.3866 & $1.3867(3)$ & $1.3866(3)$ & $1.3866(3)$ & $1.3867(3)$ & $1.3866(3)$ & $1.3866(3)$ & $1.3860(3)$ & $1.3860(3)$ & $1.3860(3)$ \\
\hline $\mathrm{C} 6-\mathrm{C} 7$ & 1.4017 & $1.4005(3)$ & $1.4005(3)$ & $1.4005(3)$ & $1.4004(3)$ & $1.4005(3)$ & $1.4005(3)$ & $1.4007(3)$ & $1.4008(3)$ & $1.4008(2)$ \\
\hline $\mathrm{C} 7-\mathrm{C} 8$ & 1.3933 & $1.3926(2)$ & $1.3925(2)$ & $1.3925(2)$ & $1.3926(2)$ & $1.3925(2)$ & $1.3925(2)$ & $1.3921(2)$ & $1.3920(2)$ & $1.3920(2)$ \\
\hline C8 - C9 & 1.4184 & $1.4145(2)$ & $1.4146(2)$ & $1.4146(2)$ & $1.4146(2)$ & $1.4146(2)$ & $1.4146(2)$ & $1.4141(2)$ & $1.4142(2)$ & $1.4142(2)$ \\
\hline C10 - C11 & 1.4013 & $1.3979(2)$ & $1.3979(2)$ & $1.3979(2)$ & $1.3979(2)$ & $1.3979(2)$ & $1.3979(2)$ & $1.3977(2)$ & $1.3977(2)$ & $1.3977(2)$ \\
\hline C10 - C15 & 1.4013 & $1.3998(2)$ & $1.3998(2)$ & $1.3998(2)$ & $1.3998(2)$ & $1.3998(2)$ & $1.3998(2)$ & $1.3989(2)$ & $1.3989(2)$ & $1.3989(2)$ \\
\hline C11 - C12 & 1.3967 & $1.3954(3)$ & $1.3954(3)$ & $1.3954(3)$ & $1.3954(3)$ & $1.3954(3)$ & $1.3954(3)$ & $1.3951(3)$ & $1.3950(3)$ & $1.3950(3)$ \\
\hline C12 - C13 & 1.3983 & $1.3990(4)$ & $1.3990(4)$ & $1.3990(4)$ & $1.3990(4)$ & $1.3990(4)$ & $1.3990(4)$ & $1.3983(3)$ & $1.3982(3)$ & $1.3982(3)$ \\
\hline $\mathrm{C} 13-\mathrm{C} 14$ & 1.3983 & $1.3962(3)$ & $1.3962(3)$ & $1.3962(3)$ & $1.3962(4)$ & $1.3963(3)$ & $1.3963(3)$ & $1.3960(3)$ & $1.3962(3)$ & $1.3962(3)$ \\
\hline C14 - C15 & 1.3973 & $1.3949(3)$ & $1.3949(3)$ & $1.3949(3)$ & $1.3949(3)$ & $1.3949(3)$ & $1.3949(3)$ & $1.3948(3)$ & $1.3948(3)$ & $1.3948(3)$ \\
\hline $\mathrm{C} 2-\mathrm{H} 2$ & 1.0888 & $1.083(5)$ & $1.083(5)$ & $1.083(5)$ & $1.12(2)$ & $1.11(2)$ & $1.11(2)$ & $1.075(7)$ & $1.076(7)$ & $1.074(7)$ \\
\hline C3 - H3 & 1.0936 & $1.083(6)$ & $1.083(6)$ & $1.083(6)$ & $1.09(2)$ & $1.09(2)$ & $1.09(2)$ & $1.084(6)$ & $1.084(6)$ & $1.084(6)$ \\
\hline C5 - H5 & 1.0914 & $1.082(6)$ & $1.083(6)$ & $1.083(6)$ & $1.06(2)$ & $1.06(2)$ & $1.06(2)$ & $1.073(6)$ & $1.075(6)$ & $1.074(6)$ \\
\hline $\mathrm{C} 6-\mathrm{H} 6$ & 1.0885 & $1.084(6)$ & $1.083(6)$ & $1.083(6)$ & $1.09(2)$ & $1.09(2)$ & $1.09(2)$ & $1.073(7)$ & $1.072(6)$ & $1.071(7)$ \\
\hline $\mathrm{C} 7-\mathrm{H} 7$ & 1.0897 & $1.083(4)$ & $1.083(4)$ & $1.083(4)$ & $1.11(2)$ & $1.11(2)$ & $1.11(2)$ & $1.080(7)$ & $1.082(7)$ & $1.081(7)$ \\
\hline C11 - H11 & 1.0912 & $1.083(6)$ & $1.084(6)$ & $1.084(6)$ & $1.13(2)$ & $1.11(2)$ & $1.13(2)$ & $1.109(7)$ & $1.110(7)$ & $1.109(7)$ \\
\hline $\mathrm{C} 12-\mathrm{H} 12$ & 1.0891 & $1.083(7)$ & $1.083(7)$ & $1.083(7)$ & $1.10(1)$ & $1.102(4)$ & $1.10(1)$ & $1.078(6)$ & $1.079(6)$ & $1.077(6)$ \\
\hline C13 - H13 & 1.0898 & $1.08(1)$ & $1.08(1)$ & $1.08(1)$ & $1.09(3)$ & $1.10(2)$ & $1.10(2)$ & $1.069(7)$ & $1.069(7)$ & $1.068(7)$ \\
\hline C14 - H14 & 1.0903 & $1.083(7)$ & $1.083(7)$ & $1.083(7)$ & $1.11(3)$ & $1.12(3)$ & $1.12(3)$ & $1.090(7)$ & $1.090(7)$ & $1.089(7)$ \\
\hline C15 - H15 & 1.0871 & $1.083(6)$ & $1.083(6)$ & $1.083(6)$ & $1.08(2)$ & $1.08(1)$ & $1.08(1)$ & $1.081(6)$ & $1.082(6)$ & $1.081(6)$ \\
\hline N1 - H1 & 1.0311 & $1.029(5)$ & $1.030(4)$ & $1.030(4)$ & $0.99(2)$ & $1.00(2)$ & $1.00(2)$ & $1.041(6)$ & $1.042(6)$ & $1.042(6)$ \\
\hline
\end{tabular}


Table 11: Angles for PT-2(Cl) (intensity cut-off: $|F| \geq 2 \sigma(|F|)$ ).

\begin{tabular}{|c|c|c|c|c|c|c|c|c|c|c|}
\hline & opt & harm & $\begin{array}{c}\mathrm{MM} \\
\text { anh, } \mathrm{n}=3\end{array}$ & anh, $\mathrm{n}=3,4$ & harm & $\begin{array}{l}\mathrm{MM}(\text { freeXH) } \\
\text { anh, n=3 }\end{array}$ & anh, $\mathrm{n}=3,4$ & harm & $\begin{array}{c}\text { HAR } \\
\text { anh, } n=3\end{array}$ & anh, $\mathrm{n}=3,4$ \\
\hline C1 C2 C3 & 120.56 & $120.30(4)$ & $120.30(4)$ & $120.30(4)$ & $120.30(4)$ & $120.30(4)$ & $120.30(4)$ & $120.29(3)$ & $120.29(3)$ & $120.29(3)$ \\
\hline $\mathrm{C} 1 \mathrm{C} 2 \mathrm{H} 2$ & 118.73 & $119.9(8)$ & $119.9(8)$ & $119.8(8)$ & $119.7(10)$ & $119.7(10)$ & $119.7(10)$ & $120.6(4)$ & $120.5(4)$ & $120.2(4)$ \\
\hline $\mathrm{C} 2 \mathrm{C} 3 \mathrm{C} 4$ & 119.57 & $119.65(4)$ & $119.65(4)$ & $119.65(4)$ & $119.65(4)$ & $119.65(4)$ & $119.65(4)$ & $119.65(3)$ & $119.65(3)$ & $119.66(3)$ \\
\hline $\mathrm{C} 2 \mathrm{C} 3 \mathrm{H} 3$ & 119.82 & $122.4(9)$ & $122.5(9)$ & $122.5(9)$ & $122(1)$ & $123(1)$ & 123(1) & $121.3(4)$ & $121.3(4)$ & $121.2(4)$ \\
\hline $\mathrm{C} 3 \mathrm{C} 2 \mathrm{H} 2$ & 120.71 & $119.8(8)$ & $119.8(8)$ & $119.9(8)$ & $120(1)$ & $120(1)$ & $120(1)$ & $119.1(4)$ & $119.2(4)$ & $119.5(4)$ \\
\hline $\mathrm{C} 3 \mathrm{C} 4 \mathrm{H} 4$ & 120.31 & $118.6(10)$ & $118.7(10)$ & $118.7(9)$ & $119(1)$ & $119(1)$ & $119(1)$ & $120.0(4)$ & $120.0(4)$ & $120.0(4)$ \\
\hline $\mathrm{C} 4 \mathrm{C} 3 \mathrm{H} 3$ & 120.61 & $117.9(8)$ & $117.8(8)$ & $117.8(8)$ & 118(1) & $118(1)$ & $118(1)$ & 119.1(4) & $119.0(4)$ & $119.1(4)$ \\
\hline $\mathrm{C} 5 \mathrm{C} 4 \mathrm{C} 3$ & 120.59 & $120.54(4)$ & $120.54(4)$ & $120.54(4)$ & $120.54(4)$ & $120.54(4)$ & $120.53(4)$ & $120.53(3)$ & $120.53(3)$ & $120.52(3)$ \\
\hline $\mathrm{C} 5 \mathrm{C} 4 \mathrm{H} 4$ & 119.11 & $120.8(10)$ & $120.7(10)$ & $120.8(9)$ & $121(1)$ & $121(1)$ & $121(1)$ & $119.4(4)$ & $119.5(4)$ & $119.5(4)$ \\
\hline C5 C6 C1 & 117.81 & $118.24(3)$ & $118.24(3)$ & $118.24(3)$ & $118.24(3)$ & $118.24(3)$ & $118.24(3)$ & $118.23(2)$ & $118.24(2)$ & $118.24(2)$ \\
\hline $\mathrm{C} 5 \mathrm{C} 7 \mathrm{C} 8$ & 121.42 & $121.13(3)$ & $121.14(3)$ & $121.13(3)$ & $121.13(3)$ & $121.13(3)$ & $121.13(3)$ & $121.15(3)$ & $121.15(3)$ & $121.14(2)$ \\
\hline C5 $\mathrm{C} 7 \mathrm{H} 7$ & 119.11 & 119.7(9) & 119.6(9) & $119.5(9)$ & $120(1)$ & $120(1)$ & $120(1)$ & $119.1(5)$ & $119.2(4)$ & $119.2(4)$ \\
\hline C6 $\mathrm{C} 1 \mathrm{C} 2$ & 121.23 & $121.16(3)$ & $121.16(3)$ & $121.16(3)$ & $121.16(3)$ & $121.16(3)$ & $121.16(3)$ & $121.17(3)$ & $121.17(3)$ & $121.17(2)$ \\
\hline C6 C5 C4 & 120.22 & $120.10(3)$ & $120.10(3)$ & $120.10(3)$ & $120.10(3)$ & $120.10(3)$ & $120.10(3)$ & $120.11(3)$ & $120.11(3)$ & $120.11(2)$ \\
\hline C6 $\mathrm{C} 5 \mathrm{C} 7$ & 117.45 & $117.65(3)$ & $117.65(3)$ & $117.65(3)$ & $117.65(3)$ & $117.65(3)$ & $117.65(3)$ & $117.64(2)$ & $117.64(2)$ & $117.64(2)$ \\
\hline C6 N1 C9 & 124.34 & $123.93(3)$ & $123.92(3)$ & $123.92(3)$ & $123.92(3)$ & $123.92(3)$ & $123.92(3)$ & $123.89(2)$ & $123.89(2)$ & $123.90(2)$ \\
\hline C6 N1 H1 & 121.09 & $121.2(8)$ & $121.2(8)$ & $121.1(8)$ & $121(1)$ & $121(1)$ & $121(1)$ & $122.1(5)$ & $122.0(5)$ & $121.5(5)$ \\
\hline C7 C5 C4 & 122.32 & $122.25(4)$ & $122.25(4)$ & $122.25(4)$ & $122.25(4)$ & $122.25(4)$ & $122.25(4)$ & $122.25(3)$ & $122.25(3)$ & $122.25(2)$ \\
\hline C7 C8 H8 & 120.65 & $121.1(9)$ & $121.2(9)$ & $121.1(9)$ & $121(1)$ & $121(1)$ & $121(1)$ & $122.6(4)$ & $122.5(4)$ & $122.5(4)$ \\
\hline $\mathrm{C} 8 \mathrm{C} 7 \mathrm{H} 7$ & 119.48 & $119.2(9)$ & $119.2(9)$ & $119.3(9)$ & 119(1) & $119(1)$ & 119(1) & $119.7(5)$ & $119.6(4)$ & $119.6(4)$ \\
\hline $\mathrm{C} 9 \mathrm{C} 8 \mathrm{C} 7$ & 121.31 & $121.15(3)$ & $121.15(3)$ & $121.15(3)$ & $121.16(3)$ & $121.15(3)$ & $121.15(3)$ & $121.17(3)$ & $121.17(3)$ & $121.18(2)$ \\
\hline C9 C8 H8 & 118.04 & $117.7(8)$ & $117.7(8)$ & $117.8(8)$ & $118(1)$ & $118(1)$ & $118(1)$ & $116.2(4)$ & $116.3(4)$ & $116.3(4)$ \\
\hline C9 N1 H1 & 114.53 & $114.8(7)$ & $114.8(7)$ & $114.9(7)$ & $115(1)$ & $115(1)$ & $115(1)$ & $114.0(5)$ & $114.0(5)$ & $114.5(5)$ \\
\hline $\mathrm{Cl} 1 \mathrm{C} 1 \mathrm{C} 2$ & 118.80 & $119.37(3)$ & $119.37(3)$ & $119.37(3)$ & $119.37(3)$ & $119.37(3)$ & $119.37(3)$ & $119.36(2)$ & $119.37(2)$ & $119.37(2)$ \\
\hline $\mathrm{Cl} 1 \mathrm{C}_{1} \mathrm{C} 6$ & 119.97 & $119.47(3)$ & $119.47(3)$ & $119.47(3)$ & $119.47(3)$ & $119.47(3)$ & $119.47(3)$ & $119.47(2)$ & $119.46(2)$ & $119.46(2)$ \\
\hline N1 C6 C1 & 122.69 & $121.96(3)$ & $121.95(3)$ & $121.95(3)$ & $121.96(3)$ & $121.95(3)$ & $121.95(3)$ & $121.93(2)$ & $121.93(2)$ & $121.93(2)$ \\
\hline N1 C6 C5 & 119.50 & $119.80(3)$ & $119.80(3)$ & $119.80(3)$ & $119.80(3)$ & $119.80(3)$ & $119.80(3)$ & $119.84(2)$ & $119.84(2)$ & $119.83(2)$ \\
\hline N1 C9 C8 & 115.97 & $116.34(3)$ & $116.34(3)$ & $116.34(3)$ & $116.33(3)$ & $116.34(3)$ & $116.34(3)$ & $116.31(2)$ & $116.31(2)$ & $116.30(2)$ \\
\hline N1 C9 O1 & 120.97 & $121.07(3)$ & $121.07(3)$ & $121.07(3)$ & $121.07(3)$ & $121.07(3)$ & $121.07(3)$ & $121.08(3)$ & $121.08(3)$ & $121.08(2)$ \\
\hline $\mathrm{O} 1 \mathrm{C} 9 \mathrm{C} 8$ & 123.07 & $122.59(4)$ & $122.59(4)$ & $122.59(4)$ & $122.60(4)$ & $122.60(4)$ & $122.59(4)$ & $122.61(3)$ & $122.61(3)$ & $122.61(2)$ \\
\hline
\end{tabular}

Table 12: Angles for PT-2(Cl) (no intensity cut-off).

\begin{tabular}{|c|c|c|c|c|c|c|c|c|c|c|}
\hline & opt & harm & $\begin{array}{c}\mathrm{MM} \\
\text { anh, } \mathrm{n}=3\end{array}$ & anh, $\mathrm{n}=3,4$ & harm & $\begin{array}{c}\mathrm{MM}(\text { freeXH }) \\
\text { anh, } \mathrm{n}=3\end{array}$ & anh, $\mathrm{n}=3,4$ & harm & $\begin{array}{c}\text { HAR } \\
\text { anh, } n=3\end{array}$ & anh, $\mathrm{n}=3,4$ \\
\hline $\mathrm{C} 1 \mathrm{C} 2 \mathrm{C} 3$ & 120.56 & $120.29(3)$ & $120.30(3)$ & $120.29(2)$ & $120.29(3)$ & $120.29(3)$ & $120.29(3)$ & $120.29(2)$ & $120.29(2)$ & $120.29(2)$ \\
\hline $\mathrm{C} 1 \mathrm{C} 2 \mathrm{H} 2$ & 118.73 & $119.2(5)$ & $119.2(5)$ & $119.3(5)$ & 119.1(8) & 119.1(8) & $119.2(8)$ & $120.5(4)$ & $120.5(4)$ & $120.2(3)$ \\
\hline $\mathrm{C} 2 \mathrm{C} 3 \mathrm{C} 4$ & 119.57 & $119.65(3)$ & $119.65(3)$ & $119.65(3)$ & $119.66(3)$ & $119.65(3)$ & $119.66(3)$ & $119.65(2)$ & $119.65(2)$ & $119.66(2)$ \\
\hline $\mathrm{C} 2 \mathrm{C} 3 \mathrm{H} 3$ & 119.82 & $122.5(6)$ & 122.6(6) & $122.6(6)$ & 122.(1) & 123.(1) & 123.(1) & $121.3(4)$ & $121.4(4)$ & $121.2(4)$ \\
\hline $\mathrm{C} 3 \mathrm{C} 2 \mathrm{H} 2$ & 120.71 & $120.5(5)$ & $120.5(5)$ & $120.4(5)$ & $120.6(9)$ & 120.6(9) & 120.6(9) & $119.2(4)$ & $119.2(4)$ & $119.5(3)$ \\
\hline $\mathrm{C} 3 \mathrm{C} 4 \mathrm{H} 4$ & 120.31 & $118.7(7)$ & $118.8(7)$ & $118.8(7)$ & 119.(1) & 119.(1) & 119.(1) & $120.0(4)$ & $120.0(4)$ & $120.1(4)$ \\
\hline $\mathrm{C} 4 \mathrm{C} 3 \mathrm{H} 3$ & 120.61 & $117.9(6)$ & 117.8(6) & $117.7(6)$ & $117.9(9)$ & $117.8(9)$ & $117.8(9)$ & $119.0(4)$ & $119.0(4)$ & $119.1(4)$ \\
\hline $\mathrm{C} 5 \mathrm{C} 4 \mathrm{C} 3$ & 120.59 & $120.53(3)$ & $120.52(3)$ & $120.52(3)$ & $120.53(3)$ & $120.53(3)$ & $120.52(3)$ & $120.53(2)$ & $120.53(2)$ & $120.52(2)$ \\
\hline $\mathrm{C} 5 \mathrm{C} 4 \mathrm{H} 4$ & 119.11 & $120.8(7)$ & $120.6(7)$ & $120.7(7)$ & 121.(1) & 121.(1) & 121.(1) & $119.4(4)$ & $119.5(4)$ & $119.4(4)$ \\
\hline C5 $\mathrm{C} 6 \mathrm{C} 1$ & 117.81 & $118.23(2)$ & $118.23(2)$ & $118.23(2)$ & $118.23(2)$ & $118.23(2)$ & $118.23(2)$ & $118.23(2)$ & $118.23(2)$ & $118.24(2)$ \\
\hline C5 $\mathrm{C} 7 \mathrm{C} 8$ & 121.42 & $121.13(2)$ & $121.13(2)$ & $121.13(2)$ & $121.12(2)$ & $121.13(2)$ & $121.12(2)$ & $121.15(2)$ & $121.15(2)$ & $121.14(2)$ \\
\hline C5 $\mathrm{C} 7 \mathrm{H} 7$ & 119.11 & $119.6(6)$ & $119.5(6)$ & $119.4(6)$ & 120.(1) & 120.(1) & 119.(1) & 119.1(4) & $119.2(4)$ & $119.2(4)$ \\
\hline $\mathrm{C} 6 \mathrm{C} 1 \mathrm{C} 2$ & 121.23 & $121.17(2)$ & $121.17(2)$ & $121.17(2)$ & $121.17(2)$ & $121.17(2)$ & $121.17(2)$ & $121.17(2)$ & $121.17(2)$ & $121.17(2)$ \\
\hline C6 C5 C4 & 120.22 & $120.11(2)$ & $120.11(2)$ & $120.11(2)$ & $120.11(2)$ & $120.11(2)$ & $120.11(2)$ & $120.11(2)$ & $120.11(2)$ & $120.11(2)$ \\
\hline C6 C5 C7 & 117.45 & $117.65(2)$ & $117.65(2)$ & $117.66(2)$ & $117.65(2)$ & $117.65(2)$ & $117.65(2)$ & $117.64(2)$ & $117.64(2)$ & $117.64(2)$ \\
\hline C6 N1 C9 & 124.34 & $123.93(2)$ & $123.93(2)$ & $123.93(2)$ & $123.93(2)$ & $123.93(2)$ & $123.92(2)$ & $123.89(2)$ & $123.89(2)$ & $123.90(2)$ \\
\hline C6 N1 H1 & 121.09 & $121.5(5)$ & $121.5(5)$ & $121.4(5)$ & 121.(1) & 121.(1) & 121.(1) & $122.1(4)$ & $122.0(4)$ & $121.5(4)$ \\
\hline $\mathrm{C} 7 \mathrm{C} 5 \mathrm{C} 4$ & 122.32 & $122.24(2)$ & $122.23(2)$ & $122.23(2)$ & $122.24(2)$ & $122.24(2)$ & $122.23(2)$ & $122.25(2)$ & $122.25(2)$ & $122.25(2)$ \\
\hline $\mathrm{C} 7 \mathrm{C} 8 \mathrm{H} 8$ & 120.65 & $121.2(5)$ & $121.2(5)$ & $121.1(5)$ & $121.4(10)$ & $121.5(10)$ & $121.4(10)$ & $122.6(4)$ & $122.6(3)$ & $122.7(3)$ \\
\hline $\mathrm{C} 8 \mathrm{C} 7 \mathrm{H} 7$ & 119.48 & $119.3(6)$ & $119.4(6)$ & $119.4(6)$ & 119.(1) & 119.(1) & 119.(1) & $119.7(4)$ & $119.6(4)$ & $119.6(4)$ \\
\hline $\mathrm{C} 9 \mathrm{C} 8 \mathrm{C} 7$ & 121.31 & $121.15(2)$ & $121.14(2)$ & $121.14(2)$ & $121.16(2)$ & $121.15(2)$ & $121.15(2)$ & $121.17(2)$ & $121.17(2)$ & $121.18(2)$ \\
\hline C9 $\mathrm{C} 8 \mathrm{H} 8$ & 118.04 & $117.7(5)$ & $117.6(5)$ & $117.7(5)$ & $117.4(8)$ & $117.4(8)$ & $117.4(8)$ & $116.2(4)$ & $116.3(3)$ & $116.2(3)$ \\
\hline C9 N1 H1 & 114.53 & $114.6(5)$ & $114.5(5)$ & $114.6(5)$ & $114.5(10)$ & $114.5(10)$ & $114.6(10)$ & $113.9(4)$ & $114.0(4)$ & $114.5(4)$ \\
\hline $\mathrm{CL} 1 \mathrm{C} 1 \mathrm{C} 2$ & 118.80 & $119.36(2)$ & $119.37(2)$ & $119.37(2)$ & $119.36(2)$ & $119.37(2)$ & $119.36(2)$ & $119.36(2)$ & $119.36(2)$ & $119.37(2)$ \\
\hline CL1 C1 C6 & 119.97 & $119.463(18)$ & $119.459(18)$ & $119.460(18)$ & $119.465(18)$ & $119.462(18)$ & $119.462(18)$ & $119.47(2)$ & $119.46(2)$ & $119.46(2)$ \\
\hline $\mathrm{N} 1 \mathrm{C} 6 \mathrm{C} 1$ & 122.69 & $121.97(2)$ & $121.97(2)$ & $121.97(2)$ & $121.97(2)$ & $121.97(2)$ & $121.96(2)$ & $121.93(2)$ & $121.93(2)$ & $121.93(2)$ \\
\hline N1 C6 C5 & 119.50 & $119.80(2)$ & $119.80(2)$ & $119.80(2)$ & $119.80(2)$ & $119.80(2)$ & $119.80(2)$ & $119.84(2)$ & $119.84(2)$ & $119.83(2)$ \\
\hline N1 C9 C8 & 115.97 & $116.34(2)$ & $116.34(2)$ & $116.35(2)$ & $116.34(2)$ & $116.34(2)$ & $116.34(2)$ & 116.31(2) & $116.31(2)$ & $116.30(2)$ \\
\hline N1 C9 O1 & 120.97 & $121.08(3)$ & $121.08(3)$ & $121.08(2)$ & $121.08(3)$ & $121.08(3)$ & $121.08(2)$ & $121.08(2)$ & $121.08(2)$ & $121.08(2)$ \\
\hline O1 C9 C8 & 123.07 & $122.58(3)$ & $122.58(3)$ & $122.58(3)$ & $122.58(3)$ & $122.58(3)$ & $122.58(3)$ & $122.61(2)$ & $122.61(2)$ & $122.61(2)$ \\
\hline
\end{tabular}


Table 13: Angles for PT-8(Br) (intensity cut-off: $|F| \geq 2 \sigma(|F|)$ ).

\begin{tabular}{|c|c|c|c|c|c|c|c|}
\hline & opt & harm & $\begin{array}{c}\text { MM } \\
\text { anh, } n=3\end{array}$ & anh, $\mathrm{n}=3,4$ & harm & $\begin{array}{c}\text { HAR } \\
\text { anh, } n=3\end{array}$ & anh, $\mathrm{n}=3,4$ \\
\hline $\mathrm{Br} 1 \mathrm{C} 1 \mathrm{C} 2$ & 118.37 & $118.90(5)$ & $118.89(5)$ & $118.90(5)$ & $118.90(4)$ & $118.89(4)$ & $118.89(4)$ \\
\hline Br1 C1 C6 & 121.39 & $119.95(5)$ & $119.95(5)$ & $119.95(5)$ & $119.94(4)$ & $119.94(4)$ & $119.94(4)$ \\
\hline C1 C2 C3 & 121.37 & $120.47(7)$ & $120.46(7)$ & $120.47(7)$ & $120.47(5)$ & $120.46(5)$ & $120.47(5)$ \\
\hline $\mathrm{C} 1 \mathrm{C} 2 \mathrm{H} 2$ & 119.79 & 119.7(6) & $119.5(6)$ & $119.5(6)$ & $117.9(8)$ & $117.8(8)$ & $117.7(8)$ \\
\hline $\mathrm{C} 2 \mathrm{C} 3 \mathrm{C} 4$ & 119.47 & $119.50(7)$ & $119.51(7)$ & $119.50(7)$ & $119.48(5)$ & $119.48(5)$ & $119.48(5)$ \\
\hline C2 C3 H3 & 120.15 & 118(1) & $118(1)$ & 118(1) & 121.3(8) & $121.3(8)$ & 121.4(8) \\
\hline $\mathrm{C} 3 \mathrm{C} 2 \mathrm{H} 2$ & 118.83 & $119.8(6)$ & $120.0(6)$ & $120.0(6)$ & $121.6(8)$ & $121.7(8)$ & $121.8(8)$ \\
\hline $\mathrm{C} 3 \mathrm{C} 4 \mathrm{H} 4$ & 120.57 & $120(1)$ & $120(1)$ & $120(1)$ & $121.5(9)$ & 121.4(9) & $121.4(9)$ \\
\hline C4 C3 H3 & 120.37 & $122(1)$ & $122(1)$ & $122(1)$ & 119.1(8) & $119.0(8)$ & 118.9(8) \\
\hline C5 C4 C3 & 120.14 & $120.63(8)$ & $120.63(7)$ & $120.63(7)$ & $120.64(5)$ & $120.63(5)$ & $120.63(5)$ \\
\hline $\mathrm{C} 5 \mathrm{C} 4 \mathrm{H} 4$ & 119.29 & 119(1) & 119(1) & 119(1) & $117.9(9)$ & $118.0(9)$ & 117.9(9) \\
\hline C5 C6 C1 & 118.89 & 118.06(5) & $118.07(5)$ & $118.07(5)$ & $118.06(5)$ & $118.07(5)$ & $118.06(5)$ \\
\hline C5 C6 N1 & 118.53 & $119.49(6)$ & $119.49(6)$ & $119.48(6)$ & $119.47(5)$ & $119.47(5)$ & $119.47(4)$ \\
\hline C5 C7 C8 & 121.61 & $121.21(6)$ & $121.22(6)$ & $121.21(6)$ & $121.21(5)$ & $121.21(5)$ & $121.21(5)$ \\
\hline C5 C7 H7 & 118.71 & 118(1) & 118(1) & 118.4(10) & 119.6(9) & 119.6(9) & 119.7(8) \\
\hline $\mathrm{C} 6 \mathrm{C} 1 \mathrm{C} 2$ & 120.25 & $121.15(7)$ & $121.15(7)$ & $121.15(6)$ & $121.16(5)$ & $121.17(5)$ & $121.16(5)$ \\
\hline $\mathrm{C} 6 \mathrm{C} 5 \mathrm{C} 4$ & 119.88 & $120.17(6)$ & $120.17(6)$ & $120.16(6)$ & $120.18(5)$ & $120.18(5)$ & $120.18(5)$ \\
\hline $\mathrm{C} 6 \mathrm{C} 5 \mathrm{C} 7$ & 118.02 & $117.79(6)$ & $117.79(6)$ & $117.80(6)$ & $117.83(5)$ & $117.82(5)$ & $117.82(5)$ \\
\hline C6 N1 C9 & 124.93 & $124.14(5)$ & $124.14(5)$ & $124.15(5)$ & $124.10(4)$ & $124.11(4)$ & $124.10(4)$ \\
\hline C6 N1 H1 & 121.1 & 120.6(8) & $120.7(8)$ & $120.9(8)$ & $122.5(9)$ & $122.6(9)$ & $122.6(9)$ \\
\hline $\mathrm{C} 7 \mathrm{C} 5 \mathrm{C} 4$ & 122.09 & $122.03(7)$ & $122.03(7)$ & $122.03(6)$ & $121.99(5)$ & $122.00(5)$ & $121.99(5)$ \\
\hline $\mathrm{C} 7 \mathrm{C} 8 \mathrm{H} 8$ & 121.11 & $123.2(7)$ & 123.3(7) & $123.1(7)$ & $119.5(8)$ & 119.7(8) & $119.5(8)$ \\
\hline $\mathrm{C} 8 \mathrm{C} 7 \mathrm{H} 7$ & 119.68 & $120(1)$ & $120(1)$ & $120.3(10)$ & $119.2(9)$ & 119.1(9) & 119.1(8) \\
\hline C8 C9 O1 & 122.86 & $122.62(7)$ & $122.62(7)$ & $122.63(6)$ & $122.61(5)$ & $122.60(5)$ & $122.61(5)$ \\
\hline $\mathrm{C} 9 \mathrm{C} 8 \mathrm{C} 7$ & 120.94 & $121.17(6)$ & $121.17(6)$ & $121.18(6)$ & $121.11(5)$ & $121.11(5)$ & $121.12(5)$ \\
\hline C9 C8 H8 & 117.94 & 115.6(7) & 115.6(7) & 115.7(7) & 119.3(8) & $119.2(8)$ & 119.4(8) \\
\hline C9 N1 H1 & 113.86 & 115.2(8) & 115.1(8) & 114.9(8) & 113.2(9) & 113.2(9) & 113.2(8) \\
\hline N1 C6 C1 & 122.58 & $122.44(7)$ & $122.44(6)$ & $122.45(6)$ & $122.46(5)$ & $122.46(4)$ & $122.46(4)$ \\
\hline N1 C9 C8 & 115.96 & $116.19(6)$ & $116.19(6)$ & $116.18(6)$ & $116.27(5)$ & $116.27(5)$ & $116.27(5)$ \\
\hline N1 C9 O1 & 121.19 & $121.19(6)$ & $121.19(6)$ & $121.19(6)$ & $121.13(5)$ & $121.13(5)$ & $121.13(5)$ \\
\hline
\end{tabular}

Table 14: Angles for PT-8(Br) (no intensity cut-off).

\begin{tabular}{|c|c|c|c|c|c|c|c|}
\hline & opt & harm & $\begin{array}{c}\mathrm{MM} \\
\text { anh, } \mathrm{n}=3\end{array}$ & anh, $\mathrm{n}=3,4$ & harm & $\begin{array}{c}\text { HAR } \\
\text { anh, } n=3\end{array}$ & anh, $\mathrm{n}=3,4$ \\
\hline $\mathrm{Br} 1 \mathrm{C} 1 \mathrm{C} 2$ & 118.37 & $118.90(4)$ & $118.89(4)$ & $118.89(4)$ & $118.90(4)$ & $118.89(4)$ & $118.89(4)$ \\
\hline Br1 C1 C6 & 121.39 & $119.95(4)$ & $119.95(4)$ & $119.95(4)$ & $119.94(3)$ & $119.95(3)$ & $119.95(3)$ \\
\hline C1 C2 C3 & 121.37 & $120.48(5)$ & $120.47(5)$ & $120.48(5)$ & $120.47(5)$ & $120.47(5)$ & $120.47(5)$ \\
\hline $\mathrm{C} 1 \mathrm{C} 2 \mathrm{H} 2$ & 119.79 & 119.6(6) & $119.5(6)$ & $119.4(5)$ & $117.9(7)$ & $117.8(7)$ & $117.7(7)$ \\
\hline $\mathrm{C} 2 \mathrm{C} 3 \mathrm{C} 4$ & 119.47 & $119.49(6)$ & $119.49(6)$ & $119.49(5)$ & $119.48(5)$ & $119.48(5)$ & $119.48(5)$ \\
\hline $\mathrm{C} 2 \mathrm{C} 3 \mathrm{H} 3$ & 120.15 & 121.(1) & 121.(1) & 121.(1) & 121.3(7) & 121.3(7) & $121.5(7)$ \\
\hline $\mathrm{C} 3 \mathrm{C} 2 \mathrm{H} 2$ & 118.83 & $119.9(6)$ & $120.0(6)$ & $120.0(6)$ & $121.6(7)$ & $121.7(7)$ & $121.8(7)$ \\
\hline $\mathrm{C} 3 \mathrm{C} 4 \mathrm{H} 4$ & 120.57 & $120.8(9)$ & $120.7(9)$ & $121.0(9)$ & $121.4(8)$ & $121.3(8)$ & $121.4(8)$ \\
\hline $\mathrm{C} 4 \mathrm{C} 3 \mathrm{H} 3$ & 120.37 & 119.(1) & 119.(1) & 119.(1) & 119.1(7) & 119.0(7) & 118.9(7) \\
\hline C5 C4 C3 & 120.14 & $120.63(5)$ & $120.62(5)$ & $120.63(5)$ & $120.64(5)$ & $120.63(5)$ & $120.63(5)$ \\
\hline $\mathrm{C} 5 \mathrm{C} 4 \mathrm{H} 4$ & 119.29 & $118.5(9)$ & $118.6(9)$ & 118.4(9) & $117.9(8)$ & 118.1(8) & $118.0(8)$ \\
\hline C5 C6 C1 & 118.89 & $118.08(4)$ & $118.07(4)$ & $118.08(4)$ & $118.07(4)$ & $118.07(4)$ & $118.07(4)$ \\
\hline C5 C6 N1 & 118.53 & $119.49(4)$ & $119.49(4)$ & $119.48(4)$ & $119.47(4)$ & $119.47(4)$ & $119.47(4)$ \\
\hline $\mathrm{C} 5 \mathrm{C} 7 \mathrm{C} 8$ & 121.61 & $121.25(5)$ & $121.25(5)$ & $121.24(5)$ & $121.21(4)$ & $121.21(4)$ & $121.21(4)$ \\
\hline $\mathrm{C} 5 \mathrm{C} 7 \mathrm{H} 7$ & 118.71 & $118.9(10)$ & $118.9(10)$ & $118.9(9)$ & 119.5(8) & 119.6(8) & 119.6(7) \\
\hline C6 C1 C2 & 120.25 & $121.15(5)$ & $121.15(5)$ & $121.16(5)$ & $121.16(4)$ & 121.16(4) & $121.16(4)$ \\
\hline C6 C5 C4 & 119.88 & $120.17(5)$ & $120.17(5)$ & $120.16(4)$ & $120.18(4)$ & $120.18(4)$ & $120.18(4)$ \\
\hline C6 C5 C7 & 118.02 & $117.79(4)$ & $117.78(4)$ & $117.79(4)$ & $117.83(4)$ & $117.83(4)$ & $117.83(4)$ \\
\hline C6 N1 C9 & 124.93 & $124.14(5)$ & $124.14(5)$ & $124.15(4)$ & $124.11(4)$ & $124.11(4)$ & $124.11(4)$ \\
\hline C6 N1 H1 & 121.19 & $121.3(7)$ & $121.4(7)$ & $121.6(7)$ & $122.4(8)$ & $122.5(8)$ & $122.4(8)$ \\
\hline $\mathrm{C} 7 \mathrm{C} 5 \mathrm{C} 4$ & 122.09 & $122.05(5)$ & $122.05(5)$ & $122.05(5)$ & $121.99(4)$ & $121.99(4)$ & $121.99(4)$ \\
\hline $\mathrm{C} 7 \mathrm{C} 8 \mathrm{H} 8$ & 121.11 & 122.1(6) & 122.1(6) & $121.9(6)$ & 119.6(7) & 119.8(7) & 119.6(7) \\
\hline $\mathrm{C} 8 \mathrm{C} 7 \mathrm{H} 7$ & 119.68 & $119.9(10)$ & $119.8(10)$ & $119.8(9)$ & $119.3(8)$ & $119.2(8)$ & 119.1(7) \\
\hline C8 C9 O1 & 122.86 & $122.63(5)$ & $122.63(5)$ & $122.64(5)$ & $122.61(4)$ & $122.61(4)$ & $122.61(4)$ \\
\hline $\mathrm{C} 9 \mathrm{C} 8 \mathrm{C} 7$ & 120.94 & $121.16(5)$ & $121.16(5)$ & $121.17(5)$ & $121.11(4)$ & $121.11(4)$ & $121.12(4)$ \\
\hline C9 C8 H8 & 117.94 & $116.8(6)$ & $116.7(6)$ & $117.0(6)$ & 119.3(7) & 119.1(7) & $119.3(7)$ \\
\hline C9 N1 H1 & 113.86 & $114.5(7)$ & $114.4(7)$ & $114.2(7)$ & 113.3(8) & 113.3(8) & $113.3(8)$ \\
\hline N1 C6 C1 & 122.58 & $122.43(4)$ & $122.43(4)$ & $122.44(4)$ & $122.46(4)$ & $122.46(4)$ & $122.46(4)$ \\
\hline $\mathrm{N} 1 \mathrm{C} 9 \mathrm{C} 8$ & 115.96 & $116.17(4)$ & $116.17(4)$ & $116.17(4)$ & $116.26(4)$ & $116.26(4)$ & $116.26(4)$ \\
\hline N1 C9 O1 & 121.19 & $121.19(5)$ & $121.20(5)$ & $121.19(5)$ & $121.13(4)$ & $121.14(4)$ & $121.13(4)$ \\
\hline
\end{tabular}


Table 15: Angles for PT-10(I) (intensity cut-off: $|F| \geq 2 \sigma(|F|)$ ).

\begin{tabular}{|c|c|c|c|c|c|c|c|}
\hline & opt & harm & $\begin{array}{c}\text { MM } \\
\text { anh, } n=3\end{array}$ & anh, $\mathrm{n}=3,4$ & harm & $\begin{array}{c}\text { HAR } \\
\text { anh, } n=3\end{array}$ & anh, $\mathrm{n}=3,4$ \\
\hline $\mathrm{C} 1 \mathrm{C} 2 \mathrm{C} 3$ & 121.09 & $120.4(1)$ & $120.4(1)$ & $120.4(1)$ & $120.40(9)$ & $120.45(8)$ & $120.44(8)$ \\
\hline $\mathrm{C} 1 \mathrm{C} 2 \mathrm{H} 2$ & 119.03 & 115(1) & 115(1) & $115.9(10)$ & 116.(1) & 116.(1) & 116.(1) \\
\hline $\mathrm{C} 1 \mathrm{C} 6 \mathrm{C} 5$ & 119.42 & $119.51(9)$ & $119.50(8)$ & $119.50(8)$ & $119.52(8)$ & $119.49(7)$ & $119.49(7)$ \\
\hline $\mathrm{C} 2 \mathrm{C} 3 \mathrm{H} 3$ & 119.48 & 123(1) & 122(1) & 121(1) & $119.5(8)$ & 118.5(8) & 118.7(8) \\
\hline C3 $\mathrm{C} 2 \mathrm{H} 2$ & 119.87 & $125(1)$ & $124.1(10)$ & $123.6(10)$ & 124.(1) & 123.(1) & 123.(1) \\
\hline C3 C4 C5 & 120.09 & $120.1(1)$ & $120.1(1)$ & $120.1(1)$ & $120.17(8)$ & $120.16(8)$ & $120.15(7)$ \\
\hline $\mathrm{C} 3 \mathrm{C} 4 \mathrm{H} 4$ & 121.55 & $116.6(8)$ & $116.1(7)$ & $116.1(7)$ & 116.(1) & $113.9(9)$ & $114.0(9)$ \\
\hline $\mathrm{C} 4 \mathrm{C} 3 \mathrm{C} 2$ & 119.99 & $120.2(2)$ & $120.2(1)$ & $120.2(1)$ & $120.30(8)$ & $120.27(8)$ & $120.27(8)$ \\
\hline $\mathrm{C} 4 \mathrm{C} 3 \mathrm{H} 3$ & 120.53 & $117(1)$ & $118(1)$ & $119(1)$ & 119.8(8) & $120.8(8)$ & 120.6(8) \\
\hline $\mathrm{C} 4 \mathrm{C} 5 \mathrm{C} 7$ & 122.20 & $122.2(1)$ & $122.2(1)$ & $122.2(1)$ & $122.31(8)$ & $122.28(7)$ & $122.27(7)$ \\
\hline $\mathrm{C} 5 \mathrm{C} 4 \mathrm{H} 4$ & 118.35 & $122.9(8)$ & $123.5(7)$ & $123.5(7)$ & 124.(1) & 125.7(9) & 125.6(9) \\
\hline C5 $\mathrm{C} 7 \mathrm{C} 8$ & 121.51 & $121.4(1)$ & $121.4(1)$ & $121.4(1)$ & $121.52(8)$ & $121.53(7)$ & $121.53(7)$ \\
\hline C5 $\mathrm{C} 7 \mathrm{H} 7$ & 118.37 & $115.2(8)$ & 114.9(7) & 115.1(7) & 113.(1) & 113.1(9) & 113.1(9) \\
\hline $\mathrm{C} 6 \mathrm{C} 1 \mathrm{C} 2$ & 119.57 & $119.9(1)$ & $119.94(9)$ & 119.94(9) & $119.91(8)$ & $119.90(7)$ & $119.92(7)$ \\
\hline C6 C5 C4 & 119.85 & 119.8(1) & $119.80(10)$ & $119.80(10)$ & $119.69(8)$ & $119.72(8)$ & $119.73(8)$ \\
\hline C6 $\mathrm{C} 5 \mathrm{C} 7$ & 117.93 & 117.98(9) & 117.98(8) & 117.99(8) & $118.00(8)$ & $118.00(7)$ & $118.00(7)$ \\
\hline C6 N1 C9 & 125.85 & 125.6(1) & $125.54(10)$ & $125.54(10)$ & $125.63(7)$ & $125.59(7)$ & $125.62(7)$ \\
\hline C6 N1 H1 & 117.94 & $122.1(8)$ & $122.4(8)$ & $122.5(8)$ & 119.(1) & 119.(1) & 119.(1) \\
\hline $\mathrm{C} 7 \mathrm{C} 8 \mathrm{H} 8$ & 121.48 & $120.8(10)$ & $123.8(9)$ & $124.5(9)$ & 124.(1) & 125.(1) & 126.(1) \\
\hline $\mathrm{C} 8 \mathrm{C} 7 \mathrm{H} 7$ & 120.12 & 123.4(8) & 123.7 & $123.5(7)$ & 125.(1) & 125.3(9) & $125.4(9)$ \\
\hline $\mathrm{C} 9 \mathrm{C} 8 \mathrm{C} 7$ & 121.2 & 121.1(1) & 121.0 & $121.0(1)$ & $120.94(8)$ & $120.89(8)$ & $120.90(8)$ \\
\hline C9 $\mathrm{C} 8 \mathrm{H} 8$ & 117.27 & $118.1(10)$ & 115.2 & $114.5(8)$ & 115.(1) & 114.(1) & 113.(1) \\
\hline C9 N1 H1 & 116.20 & $112.0(8)$ & $111.7(8)$ & $111.6(7)$ & 116.(1) & 115.(1) & 115.(1) \\
\hline $\mathrm{I} 1 \mathrm{C} 1 \mathrm{C} 2$ & 118.98 & $119.15(8)$ & $119.15(7)$ & $119.15(7)$ & $119.26(7)$ & $119.29(6)$ & $119.28(6)$ \\
\hline $\mathrm{I} 1 \mathrm{C} 1 \mathrm{C} 6$ & 121.43 & $120.91(6)$ & $120.90(5)$ & $120.90(5)$ & $120.82(6)$ & $120.80(5)$ & $120.79(5)$ \\
\hline N1 C6 C1 & 122.27 & $122.0(1)$ & $121.98(9)$ & $121.99(9)$ & $122.06(7)$ & $122.04(6)$ & $122.06(6)$ \\
\hline N1 C6 C5 & 118.31 & $118.5(1)$ & $118.51(9)$ & $118.50(9)$ & $118.42(7)$ & $118.46(7)$ & $118.45(7)$ \\
\hline N1 C9 C8 & 115.13 & 115.48(9) & $115.55(8)$ & $115.56(8)$ & $115.47(8)$ & $115.51(7)$ & $115.49(7)$ \\
\hline N1 C9 O1 & 120.32 & $120.1(1)$ & $120.1(1)$ & $120.1(1)$ & $120.17(8)$ & $120.18(7)$ & $120.19(7)$ \\
\hline $\mathrm{O} 1 \mathrm{C} 9 \mathrm{C} 8$ & 124.55 & $124.5(1)$ & $124.3(1)$ & $124.3(1)$ & $124.36(8)$ & $124.31(8)$ & $124.31(8)$ \\
\hline
\end{tabular}

Table 16: Angles for PT-10(I) (no intensity cut-off).

\begin{tabular}{|c|c|c|c|c|c|c|c|}
\hline & opt & harm & $\begin{array}{c}\text { MM } \\
\text { anh, } n=3\end{array}$ & anh, $\mathrm{n}=3,4$ & harm & $\begin{array}{c}\text { HAR } \\
\text { anh, } n=3\end{array}$ & anh, $\mathrm{n}=3,4$ \\
\hline $\mathrm{C} 1 \mathrm{C} 2 \mathrm{C} 3$ & 121.09 & $120.50(8)$ & $120.50(8)$ & $120.50(8)$ & $120.40(8)$ & $120.45(8)$ & $120.44(8)$ \\
\hline $\mathrm{C} 1 \mathrm{C} 2 \mathrm{H} 2$ & 119.03 & 116.1(9) & 116.8(8) & $116.9(8)$ & 116.(1) & 116.(1) & 116.1(9) \\
\hline C1 C6 C5 & 119.42 & $119.60(8)$ & $119.59(7)$ & $119.59(7)$ & $119.53(7)$ & $119.50(7)$ & $119.50(7)$ \\
\hline C2 C3 H3 & 119.48 & 123.(1) & 122.(1) & 121.(1) & $119.5(8)$ & $118.5(7)$ & $118.7(8)$ \\
\hline C3 C2 H2 & 119.87 & $123.4(8)$ & $122.7(8)$ & 122.6(8) & 124.(1) & 123.(1) & $123.3(9)$ \\
\hline C3 C4 C5 & 120.09 & $120.19(10)$ & $120.18(9)$ & $120.18(9)$ & $120.18(8)$ & $120.16(7)$ & $120.15(7)$ \\
\hline $\mathrm{C} 3 \mathrm{C} 4 \mathrm{H} 4$ & 121.55 & 118.1(7) & $117.0(6)$ & $116.9(6)$ & 115.9(9) & 114.0(9) & $114.0(8)$ \\
\hline $\mathrm{C} 4 \mathrm{C} 3 \mathrm{C} 2$ & 119.99 & $120.18(10)$ & $120.17(9)$ & $120.18(9)$ & $120.30(8)$ & $120.27(7)$ & $120.27(7)$ \\
\hline $\mathrm{C} 4 \mathrm{C} 3 \mathrm{H} 3$ & 120.53 & 117.(1) & 118.(1) & 119.(1) & $119.7(8)$ & $120.8(7)$ & $120.6(7)$ \\
\hline $\mathrm{C} 4 \mathrm{C} 5 \mathrm{C} 7$ & 122.20 & $122.32(10)$ & $122.30(9)$ & $122.30(9)$ & $122.31(8)$ & $122.28(7)$ & $122.27(7)$ \\
\hline $\mathrm{C} 5 \mathrm{C} 4 \mathrm{H} 4$ & 118.35 & $121.4(7)$ & $122.5(6)$ & $122.7(6)$ & 123.7(9) & $125.6(8)$ & $125.6(8)$ \\
\hline $\mathrm{C} 5 \mathrm{C} 7 \mathrm{C} 8$ & 121.51 & $121.50(10)$ & $121.52(9)$ & $121.51(9)$ & $121.53(8)$ & $121.54(7)$ & $121.53(7)$ \\
\hline C5 $\mathrm{C} 7 \mathrm{H} 7$ & 118.37 & $117.6(8)$ & $117.1(7)$ & $117.2(7)$ & 113.(1) & $113.2(9)$ & 113.2(9) \\
\hline $\mathrm{C} 6 \mathrm{C} 1 \mathrm{C} 2$ & 119.57 & $119.81(8)$ & $119.83(8)$ & $119.83(8)$ & $119.90(7)$ & $119.89(7)$ & $119.91(7)$ \\
\hline C6 C5 C4 & 119.85 & $119.72(8)$ & $119.72(7)$ & $119.72(7)$ & $119.69(8)$ & $119.72(7)$ & $119.73(7)$ \\
\hline C6 C5 C7 & 117.93 & $117.96(8)$ & $117.97(7)$ & $117.98(7)$ & $118.00(8)$ & $118.00(7)$ & $118.00(7)$ \\
\hline C6 N1 C9 & 125.85 & $125.53(8)$ & $125.53(8)$ & $125.53(8)$ & $125.63(7)$ & $125.59(6)$ & $125.63(6)$ \\
\hline C6 N1 H1 & 117.94 & 122.1(7) & $121.8(7)$ & $121.8(7)$ & 118.(1) & 119.(1) & 119.(1) \\
\hline $\mathrm{C} 7 \mathrm{C} 8 \mathrm{H} 8$ & 121.48 & $119.6(9)$ & $122.1(7)$ & $122.2(7)$ & 124.(1) & 125.(1) & 126.(1) \\
\hline $\mathrm{C} 8 \mathrm{C} 7 \mathrm{H} 7$ & 120.12 & $120.8(8)$ & $121.3(7)$ & $121.2(7)$ & 125.(1) & $125.2(9)$ & 125.3(9) \\
\hline $\mathrm{C} 9 \mathrm{C} 8 \mathrm{C} 7$ & 121.23 & $121.04(9)$ & $120.94(9)$ & $120.94(8)$ & $120.93(8)$ & $120.88(7)$ & $120.88(7)$ \\
\hline C9 C8 H8 & 117.27 & 119.3(9) & $116.9(8)$ & 116.8(8) & 115.(1) & 114.(1) & 113.(1) \\
\hline C9 N1 H1 & 116.20 & 112.1(7) & $112.4(7)$ & $112.4(7)$ & 116.(1) & 115.(1) & 115.(1) \\
\hline I1 C1 C2 & 118.98 & $119.22(6)$ & $119.22(6)$ & $119.22(6)$ & $119.27(6)$ & $119.29(6)$ & $119.28(6)$ \\
\hline I1 C1 C6 & 121.43 & $120.96(6)$ & $120.94(5)$ & $120.94(5)$ & $120.83(5)$ & $120.81(5)$ & $120.80(5)$ \\
\hline $\mathrm{N} 1 \mathrm{C} 6 \mathrm{C} 1$ & 122.27 & $121.90(8)$ & $121.91(7)$ & $121.91(7)$ & $122.05(7)$ & $122.03(6)$ & $122.05(6)$ \\
\hline N1 C6 C5 & 118.31 & $118.49(8)$ & $118.50(7)$ & $118.49(7)$ & $118.42(7)$ & $118.46(7)$ & $118.45(6)$ \\
\hline N1 C9 C8 & 115.13 & $115.46(8)$ & $115.53(7)$ & $115.53(7)$ & $115.47(8)$ & $115.51(7)$ & $115.49(7)$ \\
\hline N1 C9 O1 & 120.32 & $120.08(10)$ & 120.13(9) & 120.13(9) & $120.18(8)$ & $120.18(7)$ & $120.20(7)$ \\
\hline $\mathrm{O} 1 \mathrm{C} 9 \mathrm{C} 8$ & 124.55 & $124.45(11)$ & $124.34(10)$ & $124.34(10)$ & $124.35(8)$ & $124.30(7)$ & $124.31(7)$ \\
\hline
\end{tabular}


Table 17: Angles for PT-11(S-Ph) (intensity cut-off: $|F| \geq 2 \sigma(|F|)$ ).

\begin{tabular}{|c|c|c|c|c|c|c|c|c|c|c|}
\hline & opt & harm & $\begin{array}{c}\mathrm{MM} \\
\text { anh, } \mathrm{n}=3\end{array}$ & anh, $\mathrm{n}=3,4$ & harm & $\begin{array}{c}\mathrm{MM}(\text { freeXH }) \\
\text { anh, } \mathrm{n}=3\end{array}$ & anh, $\mathrm{n}=3,4$ & harm & $\begin{array}{c}\text { HAR } \\
\text { anh, n=3 }\end{array}$ & anh, $\mathrm{n}=3,4$ \\
\hline $\mathrm{C} 1 \mathrm{C} 2 \mathrm{C} 3$ & 121.24 & $121.14(1)$ & $121.14(1)$ & $121.14(1)$ & $121.14(1)$ & $121.14(1)$ & $121.14(1)$ & $121.16(2)$ & $121.16(2)$ & $121.16(2)$ \\
\hline $\mathrm{C} 1 \mathrm{C} 2 \mathrm{H} 2$ & 117.24 & $117.4(6)$ & $117.3(5)$ & $117.3(5)$ & $117.5(8)$ & $117.4(8)$ & $117.4(8)$ & $118.6(4)$ & $118.5(4)$ & $118.4(4)$ \\
\hline C1 N1 C9 & 124.76 & $124.42(2)$ & $124.42(2)$ & $124.42(2)$ & $124.43(1)$ & $124.42(2)$ & $124.42(2)$ & $124.42(1)$ & $124.42(1)$ & $124.42(1)$ \\
\hline C1 N1 H1 & 114.71 & $114.9(4)$ & $115.1(4)$ & $115.0(4)$ & $114.8(7)$ & $114.9(8)$ & $114.9(8)$ & $114.7(4)$ & $114.8(4)$ & $114.7(4)$ \\
\hline $\mathrm{C} 10 \mathrm{C} 11 \mathrm{C} 12$ & 119.37 & $119.36(2)$ & $119.36(2)$ & $119.36(2)$ & $119.36(2)$ & $119.36(2)$ & $119.36(2)$ & $119.33(2)$ & $119.34(2)$ & $119.34(2)$ \\
\hline C10 C11 H11 & 121.46 & $121.4(5)$ & $121.6(5)$ & $121.7(5)$ & $120.9(7)$ & $121.1(7)$ & $121.2(7)$ & $120.9(4)$ & $120.8(4)$ & $120.8(4)$ \\
\hline $\mathrm{C} 10 \mathrm{C} 15 \mathrm{C} 14$ & 120.12 & $119.99(2)$ & $120.00(2)$ & $120.00(2)$ & $119.99(2)$ & $120.00(2)$ & $120.00(2)$ & $119.97(2)$ & $119.98(2)$ & $119.98(2)$ \\
\hline C10 C15 H15 & 119.99 & $120.5(4)$ & $120.6(4)$ & $120.6(4)$ & $120.4(8)$ & $120.6(8)$ & $120.5(8)$ & $120.2(4)$ & $120.3(4)$ & $120.3(4)$ \\
\hline $\mathrm{C} 11 \mathrm{C} 10 \mathrm{C} 15$ & 119.84 & $120.09(1)$ & $120.08(1)$ & $120.08(1)$ & $120.09(1)$ & $120.09(1)$ & $120.08(1)$ & $120.11(2)$ & $120.10(2)$ & $120.10(2)$ \\
\hline C11 C10 S1 & 116.36 & $116.14(1)$ & $116.18(1)$ & $116.18(1)$ & $116.143(10)$ & $116.18(1)$ & $116.18(1)$ & $116.13(1)$ & $116.17(1)$ & $116.17(1)$ \\
\hline $\mathrm{C} 11 \mathrm{C} 12 \mathrm{C} 13$ & 121.23 & $121.02(2)$ & $121.02(2)$ & $121.02(2)$ & $121.02(2)$ & $121.02(2)$ & $121.02(2)$ & $121.04(2)$ & $121.04(2)$ & $121.04(2)$ \\
\hline C11 C12 H12 & 118.20 & $118.0(6)$ & $118.0(6)$ & $118.1(6)$ & $117.8(7)$ & $118.0(8)$ & $118.0(8)$ & $118.3(4)$ & $118.4(4)$ & $118.4(4)$ \\
\hline C12 C11 H11 & 119.16 & $119.2(5)$ & $119.0(5)$ & $119.0(5)$ & $119.7(7)$ & $119.5(8)$ & $119.5(8)$ & $119.8(4)$ & $119.8(4)$ & $119.8(4)$ \\
\hline $\mathrm{C} 12 \mathrm{C} 13 \mathrm{C} 14$ & 118.96 & $119.12(2)$ & $119.13(2)$ & $119.13(2)$ & $119.13(2)$ & $119.13(2)$ & $119.13(2)$ & $119.11(2)$ & $119.12(2)$ & $119.12(2)$ \\
\hline C12 C13 H13 & 120.42 & $120.3(5)$ & $120.3(5)$ & $120.4(5)$ & $120.1(9)$ & $120.2(9)$ & $120.2(9)$ & $120.6(4)$ & $120.7(4)$ & $120.7(4)$ \\
\hline $\mathrm{C} 13 \mathrm{C} 12 \mathrm{H} 12$ & 120.57 & $121.0(6)$ & $120.9(6)$ & $120.9(6)$ & $121.2(8)$ & $121.0(9)$ & $121.0(9)$ & $120.7(4)$ & $120.6(4)$ & $120.6(4)$ \\
\hline $\mathrm{C} 13 \mathrm{C} 14 \mathrm{C} 15$ & 120.46 & $120.41(2)$ & $120.41(2)$ & $120.40(2)$ & $120.41(2)$ & $120.40(2)$ & $120.40(2)$ & $120.43(2)$ & $120.42(2)$ & $120.42(2)$ \\
\hline $\mathrm{C} 13 \mathrm{C} 14 \mathrm{H} 14$ & 120.08 & $121.0(5)$ & $121.0(5)$ & $121.0(5)$ & $121.0(8)$ & $121.0(9)$ & $121.0(9)$ & $120.9(4)$ & $120.9(4)$ & $121.0(4)$ \\
\hline C14 C13 H13 & 120.61 & $120.5(5)$ & $120.5(5)$ & $120.5(5)$ & $120.8(9)$ & $120.6(9)$ & $120.6(9)$ & $120.2(4)$ & $120.1(4)$ & $120.2(4)$ \\
\hline C14 C15 H15 & 119.89 & $119.5(4)$ & $119.4(4)$ & $119.4(4)$ & $119.6(7)$ & $119.4(8)$ & $119.5(8)$ & $119.8(4)$ & $119.7(4)$ & $119.7(4)$ \\
\hline C15 C10 S1 & 123.77 & $123.74(1)$ & $123.72(1)$ & $123.72(1)$ & $123.75(1)$ & $123.72(1)$ & $123.72(1)$ & $123.74(1)$ & $123.71(1)$ & $123.71(1)$ \\
\hline $\mathrm{C} 15 \mathrm{C} 14 \mathrm{H} 14$ & 119.41 & $118.6(5)$ & $118.6(5)$ & $118.5(5)$ & $118.6(8)$ & $118.6(8)$ & $118.6(8)$ & $118.7(4)$ & $118.6(4)$ & $118.6(4)$ \\
\hline $\mathrm{C} 2 \mathrm{C} 1 \mathrm{~N} 1$ & 115.79 & $116.04(2)$ & $116.04(2)$ & $116.04(2)$ & $116.04(2)$ & $116.04(2)$ & $116.04(2)$ & $116.02(1)$ & $116.02(1)$ & $116.02(1)$ \\
\hline $\mathrm{C} 2 \mathrm{C} 1 \mathrm{O} 1$ & 123.57 & $123.14(2)$ & $123.14(2)$ & $123.14(2)$ & $123.14(2)$ & $123.14(2)$ & $123.14(2)$ & $123.16(2)$ & $123.16(2)$ & $123.16(2)$ \\
\hline $\mathrm{C} 2 \mathrm{C} 3 \mathrm{C} 4$ & 121.29 & $121.07(2)$ & $121.07(2)$ & $121.07(2)$ & $121.07(2)$ & $121.07(2)$ & $121.07(2)$ & $121.08(2)$ & $121.07(1)$ & $121.07(1)$ \\
\hline $\mathrm{C} 2 \mathrm{C} 3 \mathrm{H} 3$ & 120.48 & $121.6(5)$ & $121.7(5)$ & $121.7(5)$ & $122.0(8)$ & $121.9(8)$ & $121.9(8)$ & $121.5(4)$ & $121.5(4)$ & $121.5(4)$ \\
\hline $\mathrm{C} 3 \mathrm{C} 2 \mathrm{H} 2$ & 121.52 & $121.4(6)$ & $121.6(5)$ & $121.6(6)$ & $121.4(9)$ & $121.5(9)$ & $121.5(9)$ & $120.2(4)$ & $120.4(4)$ & $120.4(4)$ \\
\hline $\mathrm{C} 3 \mathrm{C} 4 \mathrm{C} 5$ & 122.15 & $122.14(2)$ & $122.14(2)$ & $122.14(2)$ & $122.14(2)$ & $122.14(2)$ & $122.14(2)$ & $122.17(2)$ & $122.16(2)$ & $122.17(1)$ \\
\hline C3 C4 C9 & 117.95 & $118.03(2)$ & $118.04(2)$ & $118.04(2)$ & $118.03(2)$ & $118.04(2)$ & $118.04(2)$ & $118.02(1)$ & $118.03(1)$ & $118.03(1)$ \\
\hline C4 C3 H3 & 118.24 & $117.4(5)$ & $117.2(5)$ & $117.3(5)$ & $117.0(6)$ & $117.0(7)$ & $117.0(7)$ & $117.4(4)$ & $117.5(4)$ & $117.5(4)$ \\
\hline C4 C5 C6 & 120.69 & $120.67(2)$ & $120.67(2)$ & $120.67(2)$ & $120.67(2)$ & $120.67(2)$ & $120.67(2)$ & $120.68(2)$ & $120.69(2)$ & $120.68(2)$ \\
\hline $\mathrm{C} 4 \mathrm{C} 5 \mathrm{H} 5$ & 118.50 & $117.9(4)$ & $117.9(4)$ & $117.9(4)$ & $118.0(7)$ & $117.9(8)$ & $117.9(8)$ & $118.5(4)$ & $118.6(4)$ & $118.5(4)$ \\
\hline $\mathrm{C} 4 \mathrm{C} 9 \mathrm{C} 8$ & 118.92 & $119.31(2)$ & $119.31(2)$ & $119.31(2)$ & $119.31(2)$ & $119.31(2)$ & $119.31(2)$ & $119.30(1)$ & $119.30(1)$ & $119.30(1)$ \\
\hline C4 C9 N1 & 118.89 & $119.23(2)$ & $119.23(2)$ & $119.23(2)$ & $119.23(2)$ & $119.23(2)$ & $119.23(2)$ & $119.23(1)$ & $119.22(1)$ & $119.22(1)$ \\
\hline $\mathrm{C} 5 \mathrm{C} 4 \mathrm{C} 9$ & 119.90 & $119.83(2)$ & $119.82(2)$ & $119.82(2)$ & $119.83(2)$ & $119.82(2)$ & $119.82(2)$ & $119.81(2)$ & $119.80(2)$ & $119.80(2)$ \\
\hline C5 C6 C7 & 119.36 & $119.31(2)$ & $119.31(2)$ & $119.31(2)$ & $119.31(2)$ & $119.31(2)$ & $119.31(2)$ & $119.34(2)$ & $119.34(2)$ & $119.34(2)$ \\
\hline C5 C6 H6 & 120.78 & $121.8(6)$ & $121.7(6)$ & $121.8(6)$ & $122.0(9)$ & $121.8(10)$ & $121.8(10)$ & $121.3(4)$ & $121.4(4)$ & $121.5(4)$ \\
\hline C6 C5 H5 & 120.81 & $121.4(4)$ & $121.4(4)$ & $121.5(4)$ & $121.3(8)$ & $121.4(9)$ & $121.5(9)$ & $120.8(4)$ & $120.7(4)$ & $120.8(4)$ \\
\hline $\mathrm{C} 6 \mathrm{C} 7 \mathrm{C} 8$ & 121.59 & $121.46(2)$ & $121.46(2)$ & $121.46(2)$ & $121.45(2)$ & $121.46(2)$ & $121.46(2)$ & $121.42(2)$ & $121.42(2)$ & $121.42(2)$ \\
\hline $\mathrm{C} 6 \mathrm{C} 7 \mathrm{H} 7$ & 120.35 & $120.0(5)$ & $119.9(4)$ & $119.9(4)$ & $120.0(8)$ & $120.0(8)$ & $120.1(8)$ & $120.0(4)$ & $120.0(4)$ & $120.0(4)$ \\
\hline $\mathrm{C} 7 \mathrm{C} 6 \mathrm{H} 6$ & 119.86 & $118.9(6)$ & $119.0(6)$ & $118.9(6)$ & $118.7(9)$ & $118.9(10)$ & $118.9(10)$ & $119.4(4)$ & $119.2(4)$ & $119.2(4)$ \\
\hline $\mathrm{C} 7 \mathrm{C} 8 \mathrm{C} 9$ & 119.50 & $119.39(1)$ & $119.39(1)$ & $119.39(1)$ & $119.39(1)$ & $119.39(1)$ & $119.39(1)$ & $119.41(1)$ & $119.41(1)$ & $119.41(1)$ \\
\hline C7 C8 S1 & 118.32 & $118.87(1)$ & $118.88(1)$ & $118.88(1)$ & $118.87(1)$ & $118.88(1)$ & $118.88(1)$ & $118.86(1)$ & $118.87(1)$ & $118.87(1)$ \\
\hline $\mathrm{C} 8 \mathrm{C} 7 \mathrm{H} 7$ & 118.06 & $118.6(5)$ & $118.6(4)$ & $118.6(4)$ & $118.5(7)$ & $118.5(7)$ & $118.5(7)$ & $118.6(4)$ & $118.5(4)$ & $118.6(4)$ \\
\hline C8 C9 N1 & 122.18 & $121.45(1)$ & $121.45(1)$ & $121.45(1)$ & $121.45(1)$ & $121.45(1)$ & $121.45(1)$ & $121.47(1)$ & $121.47(1)$ & $121.47(1)$ \\
\hline C8 S1 C10 & 103.87 & $103.379(7)$ & $103.457(9)$ & $103.457(9)$ & $103.379(7)$ & $103.457(9)$ & $103.457(9)$ & $103.387(8)$ & $103.461(9)$ & $103.460(9)$ \\
\hline C9 C8 S1 & 121.99 & $121.56(1)$ & $121.55(1)$ & $121.55(1)$ & $121.56(1)$ & $121.55(1)$ & $121.55(1)$ & $121.55(1)$ & $121.54(1)$ & $121.54(1)$ \\
\hline C9 N1 H1 & 120.52 & $120.6(4)$ & $120.5(4)$ & $120.5(4)$ & $120.8(9)$ & $120.6(9)$ & $120.6(9)$ & $120.8(4)$ & $120.8(4)$ & $120.9(4)$ \\
\hline $\mathrm{N} 1 \mathrm{C} 1 \mathrm{O} 1$ & 120.64 & $120.82(2)$ & $120.82(2)$ & $120.82(2)$ & $120.82(2)$ & $120.82(2)$ & $120.82(2)$ & $120.82(1)$ & $120.82(1)$ & $120.81(1)$ \\
\hline
\end{tabular}


Table 18: Angles for PT-11(S-Ph) (no intensity cut-off).

\begin{tabular}{|c|c|c|c|c|c|c|c|c|c|c|}
\hline & opt & harm & $\begin{array}{c}\mathrm{MM} \\
\text { anh, } \mathrm{n}=3\end{array}$ & anh, $\mathrm{n}=3,4$ & harm & $\begin{array}{c}\mathrm{MM}(\text { freeXH }) \\
\text { anh, } \mathrm{n}=3\end{array}$ & anh, $\mathrm{n}=3,4$ & harm & $\begin{array}{c}\text { HAR } \\
\text { anh, } n=3\end{array}$ & anh, $\mathrm{n}=3,4$ \\
\hline $\mathrm{C} 1 \mathrm{C} 2 \mathrm{C} 3$ & 121.24 & $121.148(15)$ & $121.149(14)$ & $121.149(14)$ & $121.147(15)$ & $121.149(14)$ & $121.148(14)$ & $121.16(2)$ & $121.16(2)$ & $121.16(2)$ \\
\hline $\mathrm{C} 1 \mathrm{C} 2 \mathrm{H} 2$ & 117.24 & $117.4(5)$ & $117.3(5)$ & $117.3(5)$ & $117.4(8)$ & $117.3(8)$ & $117.3(8)$ & $118.6(4)$ & $118.5(4)$ & $118.4(4)$ \\
\hline C1 N1 C9 & 124.76 & $124.423(13)$ & $124.421(13)$ & $124.421(13)$ & $124.423(13)$ & $124.422(13)$ & $124.422(13)$ & $124.42(1)$ & $124.42(1)$ & $124.42(1)$ \\
\hline C1 N1 H1 & 114.71 & $115.0(4)$ & $115.1(4)$ & $115.1(4)$ & $114.9(8)$ & $115.0(8)$ & $115.0(8)$ & $114.7(4)$ & $114.8(4)$ & $114.7(4)$ \\
\hline $\mathrm{C} 10 \mathrm{C} 11 \mathrm{C} 12$ & 119.37 & $119.347(16)$ & $119.350(15)$ & $119.351(15)$ & $119.347(16)$ & $119.351(15)$ & $119.351(15)$ & $119.33(2)$ & $119.34(2)$ & $119.34(2)$ \\
\hline C10 C11 H11 & 121.46 & $120.7(5)$ & $120.9(5)$ & $120.9(5)$ & $120.5(7)$ & $120.7(7)$ & $120.7(7)$ & $120.8(4)$ & $120.8(4)$ & $120.8(4)$ \\
\hline $\mathrm{C} 10 \mathrm{C} 15 \mathrm{C} 14$ & 120.12 & $119.990(17)$ & $119.993(16)$ & $119.993(16)$ & 119.991(17) & $119.994(16)$ & 119.994(16) & $119.97(2)$ & $119.98(2)$ & $119.98(2)$ \\
\hline C10 C15 H15 & 119.99 & $120.3(4)$ & $120.4(4)$ & $120.4(4)$ & $120.3(7)$ & $120.4(7)$ & $120.4(7)$ & $120.2(3)$ & 120.3(3) & $120.3(3)$ \\
\hline C11 C10 C15 & 119.84 & $120.093(15)$ & $120.089(14)$ & $120.089(14)$ & $120.093(15)$ & $120.089(14)$ & $120.089(14)$ & $120.11(2)$ & $120.10(2)$ & $120.10(2)$ \\
\hline C11 C10 S1 & 116.36 & $116.137(13)$ & $116.167(13)$ & $116.168(13)$ & $116.136(12)$ & $116.166(13)$ & $116.167(13)$ & $116.13(1)$ & $116.17(1)$ & $116.17(1)$ \\
\hline C11 C12 C13 & 121.23 & $121.006(19)$ & $121.007(19)$ & $121.007(19)$ & $121.006(19)$ & $121.007(19)$ & $121.007(19)$ & 121.04(2) & 121.03(2) & $121.03(2)$ \\
\hline C11 C12 H12 & 118.20 & $118.2(5)$ & $118.3(5)$ & $118.3(5)$ & 118.1(8) & $118.3(7)$ & $118.3(7)$ & $118.3(4)$ & 118.3(4) & $118.4(4)$ \\
\hline C12 C11 H11 & 119.16 & $120.0(5)$ & $119.8(5)$ & 119.7(5) & $120.1(7)$ & 119.9(7) & $119.9(7)$ & 119.8(4) & $119.8(4)$ & $119.8(4)$ \\
\hline $\mathrm{C} 12 \mathrm{C} 13 \mathrm{C} 14$ & 118.96 & $119.14(2)$ & $119.14(2)$ & $119.14(2)$ & $119.14(2)$ & $119.14(2)$ & $119.14(2)$ & $119.11(2)$ & $119.12(2)$ & $119.12(2)$ \\
\hline C12 C13 H13 & 120.42 & $120.0(5)$ & $120.1(5)$ & $120.1(5)$ & $119.9(9)$ & $120.0(9)$ & $120.1(9)$ & $120.6(4)$ & $120.7(4)$ & $120.7(4)$ \\
\hline C13 C12 H12 & 120.57 & $120.8(5)$ & $120.7(5)$ & $120.7(5)$ & $120.9(9)$ & $120.7(8)$ & $120.7(8)$ & $120.7(4)$ & $120.6(4)$ & $120.6(4)$ \\
\hline $\mathrm{C} 13 \mathrm{C} 14 \mathrm{C} 15$ & 120.46 & $120.413(19)$ & $120.410(19)$ & $120.409(19)$ & $120.411(19)$ & $120.408(19)$ & $120.408(19)$ & $120.43(2)$ & $120.42(2)$ & $120.42(2)$ \\
\hline C13 C14 H14 & 120.08 & $120.9(5)$ & $120.9(5)$ & $121.0(5)$ & $120.9(9)$ & $120.9(9)$ & $121.0(9)$ & $120.9(4)$ & $120.9(4)$ & $121.0(4)$ \\
\hline C14 C13 H13 & 120.61 & $120.9(5)$ & $120.7(4)$ & $120.7(4)$ & $121.0(9)$ & $120.8(9)$ & $120.8(9)$ & $120.2(4)$ & $120.1(4)$ & $120.2(4)$ \\
\hline C14 C15 H15 & 119.89 & $119.7(4)$ & $119.6(4)$ & $119.6(4)$ & $119.7(8)$ & $119.6(8)$ & $119.6(8)$ & $119.8(3)$ & $119.7(3)$ & $119.7(3)$ \\
\hline C15 C10 S1 & 123.77 & $123.750(12)$ & $123.724(12)$ & $123.723(12)$ & $123.750(12)$ & $123.724(12)$ & $123.724(12)$ & $123.74(1)$ & 123.71(1) & $123.71(1)$ \\
\hline C15 C14 H14 & 119.41 & $118.7(5)$ & $118.7(5)$ & $118.6(5)$ & $118.6(8)$ & $118.6(8)$ & $118.6(8)$ & $118.7(4)$ & $118.6(4)$ & $118.6(4)$ \\
\hline $\mathrm{C} 2 \mathrm{C} 1 \mathrm{~N} 1$ & 115.79 & $116.035(13)$ & $116.035(13)$ & $116.035(13)$ & $116.035(13)$ & $116.035(13)$ & $116.035(13)$ & $116.02(1)$ & $116.02(1)$ & 116.02(1) \\
\hline $\mathrm{C} 2 \mathrm{C} 1 \mathrm{O} 1$ & 123.57 & $123.139(16)$ & $123.139(16)$ & $123.140(16)$ & $123.139(16)$ & $123.139(16)$ & $123.140(16)$ & $123.15(2)$ & $123.16(1)$ & $123.16(1)$ \\
\hline $\mathrm{C} 2 \mathrm{C} 3 \mathrm{C} 4$ & 121.29 & $121.060(16)$ & $121.059(15)$ & $121.059(15)$ & $121.060(16)$ & $121.059(15)$ & $121.059(15)$ & $121.08(1)$ & $121.07(1)$ & $121.07(1)$ \\
\hline $\mathrm{C} 2 \mathrm{C} 3 \mathrm{H} 3$ & 120.48 & $122.0(4)$ & $121.9(4)$ & $121.9(4)$ & $122.1(8)$ & $122.0(8)$ & $122.0(8)$ & 121.6(4) & $121.5(4)$ & $121.5(4)$ \\
\hline $\mathrm{C} 3 \mathrm{C} 2 \mathrm{H} 2$ & 121.52 & $121.4(5)$ & $121.6(5)$ & $121.5(5)$ & $121.4(9)$ & $121.5(9)$ & $121.5(9)$ & 120.2(4) & 120.4(4) & $120.4(4)$ \\
\hline C3 C4 C5 & 122.15 & $122.140(16)$ & $122.142(16)$ & $122.142(16)$ & $122.139(16)$ & $122.142(16)$ & $122.142(16)$ & $122.17(1)$ & $122.16(1)$ & $122.16(1)$ \\
\hline C3 C4 C9 & 117.95 & $118.024(13)$ & $118.027(13)$ & $118.027(13)$ & $118.024(13)$ & $118.027(13)$ & $118.027(13)$ & $118.02(1)$ & $118.03(1)$ & $118.03(1)$ \\
\hline $\mathrm{C} 4 \mathrm{C} 3 \mathrm{H} 3$ & 118.24 & $116.9(5)$ & $117.0(4)$ & $117.0(4)$ & $116.9(7)$ & $116.9(7)$ & $116.9(7)$ & $117.3(4)$ & $117.4(4)$ & $117.4(4)$ \\
\hline $\mathrm{C} 4 \mathrm{C} 5 \mathrm{C} 6$ & 120.69 & $120.666(16)$ & $120.669(16)$ & $120.669(16)$ & $120.666(16)$ & $120.669(16)$ & $120.669(16)$ & $120.68(2)$ & $120.69(2)$ & $120.68(1)$ \\
\hline $\mathrm{C} 4 \mathrm{C} 5 \mathrm{H} 5$ & 118.50 & $118.5(4)$ & $118.4(4)$ & $118.4(4)$ & $118.5(8)$ & $118.4(8)$ & $118.4(8)$ & $118.4(4)$ & $118.6(4)$ & $118.5(4)$ \\
\hline $\mathrm{C} 4 \mathrm{C} 9 \mathrm{C} 8$ & 118.92 & $119.296(13)$ & $119.299(13)$ & $119.298(13)$ & $119.296(13)$ & $119.299(13)$ & $119.298(13)$ & $119.30(1)$ & $119.30(1)$ & $119.30(1)$ \\
\hline $\mathrm{C} 4 \mathrm{C} 9 \mathrm{~N} 1$ & 118.89 & $119.246(13)$ & $119.243(12)$ & $119.244(12)$ & $119.246(13)$ & $119.244(13)$ & $119.244(12)$ & $119.23(1)$ & $119.22(1)$ & $119.22(1)$ \\
\hline $\mathrm{C} 5 \mathrm{C} 4 \mathrm{C} 9$ & 119.90 & $119.836(14)$ & $119.830(13)$ & 119.831(13) & $119.837(14)$ & $119.831(13)$ & $119.831(13)$ & $119.81(2)$ & $119.80(2)$ & $119.80(1)$ \\
\hline $\mathrm{C} 5 \mathrm{C} 6 \mathrm{C} 7$ & 119.36 & $119.325(17)$ & $119.325(17)$ & $119.325(17)$ & $119.324(17)$ & $119.324(17)$ & $119.324(17)$ & $119.34(2)$ & $119.34(2)$ & $119.34(2)$ \\
\hline C5 C6 H6 & 120.78 & $121.4(5)$ & $121.2(5)$ & $121.2(5)$ & $121.4(9)$ & $121.2(9)$ & $121.2(9)$ & $121.3(4)$ & $121.4(4)$ & $121.5(4)$ \\
\hline C6 C5 H5 & 120.81 & $120.9(4)$ & $120.9(4)$ & $121.0(4)$ & $120.9(9)$ & $120.9(9)$ & $121.0(9)$ & $120.9(4)$ & $120.8(4)$ & $120.9(4)$ \\
\hline $\mathrm{C} 6 \mathrm{C} 7 \mathrm{C} 8$ & 121.59 & $121.454(15)$ & $121.454(15)$ & $121.454(15)$ & $121.455(15)$ & $121.455(15)$ & $121.455(15)$ & $121.42(2)$ & $121.42(2)$ & $121.42(2)$ \\
\hline C6 $\mathrm{C} 7 \mathrm{H} 7$ & 120.35 & $119.6(5)$ & $119.7(5)$ & $119.7(5)$ & $119.7(8)$ & $119.8(8)$ & $119.8(8)$ & 119.9(4) & $120.0(4)$ & $120.0(4)$ \\
\hline $\mathrm{C} 7 \mathrm{C} 6 \mathrm{H} 6$ & 119.86 & $119.3(5)$ & $119.4(5)$ & $119.4(5)$ & $119.3(9)$ & $119.4(9)$ & $119.4(9)$ & $119.3(4)$ & $119.2(4)$ & $119.2(4)$ \\
\hline $\mathrm{C} 7 \mathrm{C} 8 \mathrm{C} 9$ & 119.50 & $119.384(14)$ & $119.383(14)$ & 119.385(14) & $119.383(14)$ & 119.383(14) & $119.384(14)$ & 119.41(1) & 119.41(1) & 119.41(1) \\
\hline C7 C8 S1 & 118.32 & $118.870(11)$ & $118.880(12)$ & $118.879(12)$ & $118.870(11)$ & $118.880(12)$ & $118.879(12)$ & $118.86(1)$ & $118.87(1)$ & $118.87(1)$ \\
\hline $\mathrm{C} 8 \mathrm{C} 7 \mathrm{H} 7$ & 118.06 & $118.9(5)$ & $118.9(5)$ & $118.9(5)$ & $118.9(8)$ & $118.8(7)$ & $118.8(7)$ & 118.6(4) & 118.6(4) & $118.6(4)$ \\
\hline C8 C9 N1 & 122.18 & $121.454(13)$ & $121.453(13)$ & $121.454(13)$ & $121.454(13)$ & $121.453(13)$ & $121.453(13)$ & $121.46(1)$ & $121.47(1)$ & 121.47(1) \\
\hline C8 S1 C10 & 103.87 & $103.382(8)$ & $103.455(9)$ & $103.455(9)$ & $103.382(8)$ & $103.455(9)$ & $103.455(9)$ & $103.387(7)$ & $103.461(9)$ & $103.460(9)$ \\
\hline C9 C8 S1 & 121.99 & $121.560(10)$ & $121.555(11)$ & $121.554(11)$ & $121.561(10)$ & $121.556(11)$ & $121.555(11)$ & $121.55(1)$ & $121.54(1)$ & $121.54(1)$ \\
\hline C9 N1 H1 & 120.52 & $120.6(4)$ & $120.5(3)$ & $120.5(3)$ & $120.7(8)$ & $120.6(8)$ & $120.6(8)$ & $120.8(4)$ & $120.8(4)$ & $120.9(4)$ \\
\hline $\mathrm{N} 1 \mathrm{C} 1 \mathrm{O} 1$ & 120.64 & $120.821(15)$ & $120.822(15)$ & $120.820(15)$ & $120.821(15)$ & $120.822(15)$ & $120.820(15)$ & $120.82(1)$ & $120.81(1)$ & $120.81(1)$ \\
\hline
\end{tabular}

Table 19: Anharmonic Gram-Charlier coefficients obtained in 3rd-order-only anharmonic thermal motion refinement (intensity cut-off: $|F| \geq 2 \sigma(|F|))$. Values higher than three standard uncertainties highlighted with cyan.

\begin{tabular}{|c|c|c|c|c|c|c|c|c|c|c|}
\hline & \multicolumn{3}{|c|}{ PT-11(S-Ph) } & \multicolumn{3}{|c|}{$\mathrm{PT}-2(\mathrm{Cl})$} & \multicolumn{2}{|c|}{ PT-8(Br) } & \multicolumn{2}{|c|}{ PT-10(I) } \\
\hline & HAR & $\mathrm{MM}$ & $\mathrm{MM}($ freeXH $)$ & HAR & $\mathrm{MM}$ & $\mathrm{MM}($ freeXH) & HAR & $\mathrm{MM}$ & HAR & $\mathrm{MM}$ \\
\hline$U_{111}$ & $0.00(3)$ & $-0.00002(2)$ & $-0.00002(2)$ & $0.000018(9)$ & $0.00004(1)$ & $0.00004(1)$ & $-0.000002(7)$ & $0.000008(8)$ & $-0.0010(1)$ & $-0.0009(1)$ \\
\hline$U_{112}$ & $0.0(2)$ & $-0.00005(1)$ & $-0.00005(1)$ & $0.00005(1)$ & $0.00013(4)$ & $0.00013(4)$ & $0.00004(1)$ & $0.00011(4)$ & $0.00013(2)$ & $0.00050(5)$ \\
\hline$U_{113}$ & $0.0(2)$ & $0.00025(3)$ & $0.00025(3)$ & $0.000017(5)$ & $0.00004(1)$ & $0.00004(1)$ & $0.000007(3)$ & $0.000003(9)$ & $-0.00005(3)$ & $0.00013(8)$ \\
\hline$U_{122}$ & $0.0(2)$ & $-0.000016(4)$ & $-0.000016(4)$ & $0.00004(3)$ & $-0.0000(1)$ & $-0.0000(1)$ & $-0.00003(3)$ & $-0.00020(9)$ & $-0.000029(4)$ & $-0.00009(1)$ \\
\hline$U_{123}$ & $0.00(3)$ & $0.00005(1)$ & $0.00005(1)$ & $0.000020(8)$ & $-0.00004(4)$ & $-0.00004(4)$ & $0.000013(6)$ & $-0.00003(3)$ & $0.000027(4)$ & $0.00012(2)$ \\
\hline$U_{133}$ & $0.0(2)$ & $0.00007(2)$ & $0.00007(2)$ & $0.000004(4)$ & $-0.000022(9)$ & $-0.000022(9)$ & $-0.000004(3)$ & $-0.000020(6)$ & $-0.00001(1)$ & $0.00000(3)$ \\
\hline$U_{222}$ & $0.00(1)$ & $0.000005(1)$ & $0.000005(1)$ & $0.0002(2)$ & $0.0001(2)$ & $0.0001(2)$ & $-0.0001(2)$ & $-0.0000(2)$ & $0.000043(1)$ & $0.000047(1)$ \\
\hline$U_{223}$ & $0.0(2)$ & $0.000032(3)$ & $0.000032(3)$ & $-0.00014(3)$ & $-0.00029(7)$ & $-0.00029(7)$ & $-0.00015(2)$ & $-0.00041(6)$ & $0.000000(1)$ & $0.000009(4)$ \\
\hline$U_{233}$ & $0.0(2)$ & $0.000001(7)$ & $0.000001(7)$ & $-0.000013(8)$ & $-0.00007(2)$ & $-0.00007(2)$ & $-0.000016(6)$ & $-0.00007(2)$ & $0.000014(2)$ & $0.000043(7)$ \\
\hline$U_{333}$ & $0.00(2)$ & $0.00009(1)$ & $0.00009(1)$ & $-0.000020(4)$ & $-0.000010(3)$ & $-0.000010(3)$ & $-0.000010(3)$ & $-0.000003(2)$ & $0.000059(8)$ & $0.000067(7)$ \\
\hline
\end{tabular}


Table 20: Anharmonic Gram-Charlier coefficients obtained in 3rd-order-only anharmonic thermal motion refinement (no intensity cut-off). Values higher than three standard uncertainties highlighted with cyan.

\begin{tabular}{|c|c|c|c|c|c|c|c|c|c|c|}
\hline & \multicolumn{3}{|c|}{ PT-11(S-Ph) } & \multicolumn{3}{|c|}{$\mathrm{PT}-2(\mathrm{Cl})$} & \multicolumn{2}{|c|}{ PT-8(Br) } & \multicolumn{2}{|c|}{ PT-10(I) } \\
\hline & HAR & $\mathrm{MM}$ & $\mathrm{MM}($ freeXH) & HAR & MM & $\mathrm{MM}($ freeXH) & HAR & $\mathrm{MM}$ & HAR & $\mathrm{MM}$ \\
\hline$U_{111}$ & $0.00(3)$ & $-0.00002(1)$ & $-0.00002(2)$ & $0.000017(8)$ & $0.00003(6)$ & $0.00003(1)$ & $-0.000003(6)$ & $0.00001(8)$ & $-0.0010(1)$ & $-0.0009(1)$ \\
\hline$U_{112}$ & $0.0(2)$ & $-0.00004(9)$ & $-0.00004(1)$ & $0.00005(1)$ & $0.0001(3)$ & $0.0001(4)$ & $0.00004(1)$ & $0.0001(4)$ & $0.00013(2)$ & $0.0005(5)$ \\
\hline$U_{113}$ & $0.0(2)$ & $-0.00002(3)$ & $-0.00002(4)$ & $0.000017(4)$ & $0.00005(8)$ & $0.00006(9)$ & $0.000007(3)$ & $-0.0002(9)$ & $-0.00006(3)$ & $-0.00009(1)$ \\
\hline$U_{122}$ & $0.0(2)$ & $0.0002(1)$ & $0.0002(3)$ & $0.00004(3)$ & $0.00004(6)$ & $0.00004(1)$ & $-0.00003(3)$ & $0.000005(9)$ & $-0.000029(3)$ & $0.0002(8)$ \\
\hline$U_{123}$ & $0.00(3)$ & $0.00005(1)$ & $0.00005(1)$ & $0.000019(7)$ & $-0.00004(3)$ & $-0.00004(4)$ & $0.000012(5)$ & $-0.00004(3)$ & $0.000027(4)$ & $0.0001(2)$ \\
\hline$U_{133}$ & $0.0(2)$ & $0.000029(3)$ & $0.00003(3)$ & $0.000004(3)$ & $-0.0003(5)$ & $-0.0003(6)$ & $-0.000004(2)$ & $-0.0004(6)$ & $-0.00001(1)$ & $0.000008(4)$ \\
\hline$U_{222}$ & $0.00(1)$ & $0.000005(1)$ & $0.000005(1)$ & $0.0002(1)$ & $0.0001(9)$ & $0.0001(2)$ & $-0.0001(1)$ & $-0.0001(2)$ & $0.000044(1)$ & $0.00005(1)$ \\
\hline$U_{223}$ & $0.0(1)$ & $0.00004(2)$ & $0.00004(2)$ & $-0.00013(2)$ & $-0.000021(9)$ & $-0.00002(8)$ & $-0.00016(2)$ & $-0.00002(6)$ & $0.000000(1)$ & $0.00001(3)$ \\
\hline$U_{233}$ & $0.0(1)$ & $0.000004(6)$ & $0.000004(7)$ & $-0.000013(7)$ & $-0.0001(1)$ & $-0.00006(2)$ & $-0.000015(5)$ & $-0.00007(2)$ & $0.000015(2)$ & $0.00004(6)$ \\
\hline$U_{333}$ & $0.00(2)$ & $0.00007(6)$ & $0.00007(1)$ & $-0.000019(4)$ & $-0.00001(2)$ & $-0.000009(3)$ & $-0.000011(3)$ & $0.000003(2)$ & $0.000059(8)$ & $0.00007(7)$ \\
\hline
\end{tabular}

Table 21: Anharmonic Gram-Charlier coefficients obtained in 3rd and 4th order anharmonic thermal motion refinement (intensity cut-off: $|F| \geq 2 \sigma(|F|)$ ). Values higher than three standard uncertainties highlighted with cyan.

\begin{tabular}{|c|c|c|c|c|c|c|c|c|c|c|}
\hline & \multicolumn{3}{|c|}{$\mathrm{PT}-11(\mathrm{~S}-\mathrm{Ph})$} & \multicolumn{3}{|c|}{$\mathrm{PT}-2(\mathrm{Cl})$} & \multicolumn{2}{|c|}{ PT-8(Br) } & \multicolumn{2}{|c|}{ PT-10(I) } \\
\hline & HAR & MM & $\mathrm{MM}($ freeXH) & HAR & MM & $\mathrm{MM}($ freeXH) & HAR & $\mathrm{MM}$ & HAR & MM \\
\hline$U_{111}$ & $0.00(3)$ & $-0.00002(2)$ & $-0.00002(2)$ & $0.000019(8)$ & $0.00004(1)$ & $0.00004(1)$ & $0.000000(7)$ & $0.000010(8)$ & $-0.0009(1)$ & $-0.0009(2)$ \\
\hline$U_{112}$ & $0.0(2)$ & $-0.00005(1)$ & $0.000006(1)$ & $0.00005(1)$ & $0.00013(4)$ & $0.0002(2)$ & $0.00004(1)$ & $0.00011(4)$ & $0.00013(2)$ & $0.00051(5)$ \\
\hline$U_{113}$ & $0.0(2)$ & $0.00025(3)$ & $0.00009(1)$ & $0.000016(4)$ & $0.00003(1)$ & $-0.000010(3)$ & $0.000007(3)$ & $0.000002(9)$ & $-0.00005(3)$ & $0.00013(8)$ \\
\hline$U_{122}$ & $0.0(2)$ & $-0.000016(4)$ & $-0.00005(1)$ & $0.00003(3)$ & $-0.0000(1)$ & $0.00013(4)$ & $-0.00002(3)$ & $-0.00019(9)$ & $-0.000028(3)$ & $-0.00010(1)$ \\
\hline$U_{123}$ & $0.00(3)$ & $0.00005(1)$ & $-0.000016(4)$ & $0.000019(7)$ & $-0.00004(4)$ & $-0.0000(1)$ & $0.000013(6)$ & $-0.00003(3)$ & $0.000026(4)$ & $0.00012(2)$ \\
\hline$U_{133}$ & $0.0(2)$ & $0.00007(2)$ & $0.00025(3)$ & $0.000005(3)$ & $-0.000021(8)$ & $0.00003(1)$ & $-0.000004(2)$ & $-0.000020(6)$ & $-0.00001(1)$ & $0.00000(3)$ \\
\hline$U_{222}$ & $0.00(1)$ & $0.000005(1)$ & $0.00007(2)$ & $0.0002(1)$ & $0.0002(2)$ & $-0.000021(8)$ & $-0.0001(2)$ & $0.0000(2)$ & $0.000042(1)$ & $0.000048(1)$ \\
\hline$U_{223}$ & $0.0(2)$ & $0.000033(3)$ & $0.000033(3)$ & $-0.00013(2)$ & $-0.00029(7)$ & $-0.00029(7)$ & $-0.00015(2)$ & $-0.00041(6)$ & $-0.000001(1)$ & $0.000010(4)$ \\
\hline$U_{233}$ & $0.0(1)$ & $0.000001(7)$ & $0.000001(7)$ & $-0.000013(7)$ & $-0.00007(2)$ & $-0.00007(2)$ & $-0.000015(6)$ & $-0.00007(2)$ & $0.000014(2)$ & $0.000046(7)$ \\
\hline$U_{333}$ & $0.00(2)$ & $0.00009(1)$ & $0.00005(1)$ & $-0.000017(4)$ & $-0.000010(3)$ & $-0.00004(4)$ & $-0.000010(3)$ & $-0.000003(2)$ & $0.000058(8)$ & $0.000071(8)$ \\
\hline$U_{1111}$ & $0.0(1)$ & $0.00002(2)$ & $0.00002(2)$ & $-0.000061(4)$ & $-0.000046(6)$ & $-0.000046(6)$ & $-0.000024(3)$ & $-0.000037(3)$ & $-0.0023(2)$ & $0.0005(2)$ \\
\hline$U_{1112}$ & $0 .(2)$ & $-0.000001(9)$ & $0.000001(0)$ & $-0.000001(5)$ & $0.00002(3)$ & $-0.0008(2)$ & $-0.000014(5)$ & $-0.00007(2)$ & $-0.00004(2)$ & $-0.00012(9)$ \\
\hline$U_{1113}$ & $0 .(2)$ & $-0.00002(3)$ & $-0.000007(9)$ & $-0.000027(2)$ & $-0.000067(7)$ & $-0.000009(1)$ & $-0.000006(1)$ & $-0.000029(5)$ & $-0.00014(4)$ & $0.0003(1)$ \\
\hline$U_{1122}$ & $0.00(8)$ & $0.000000(4)$ & $-0.000001(9)$ & $-0.000070(9)$ & $0.00012(7)$ & $0.00002(3)$ & $-0.000046(8)$ & $-0.00022(6)$ & $-0.000022(3)$ & $0.00016(2)$ \\
\hline$U_{1123}$ & $0 .(1)$ & $-0.00002(1)$ & $-0.00002(3)$ & $-0.000002(3)$ & $-0.00000(3)$ & $-0.000067(7)$ & $-0.000001(3)$ & $0.00002(3)$ & $0.000001(5)$ & $0.00004(6)$ \\
\hline$U_{1133}$ & $0.0(2)$ & $-0.00003(3)$ & $0.000000(4)$ & $-0.000022(1)$ & $-0.000056(6)$ & $0.00012(7)$ & $-0.0000063(8)$ & $-0.000043(4)$ & $-0.00010(1)$ & $0.00043(7)$ \\
\hline$U_{1222}$ & $0 .(1)$ & $-0.000004(1)$ & $-0.00002(1)$ & $0.00006(3)$ & $0.0005(2)$ & $-0.00001(3)$ & $0.00007(4)$ & $0.0005(2)$ & $-0.000002(1)$ & $-0.000009(4)$ \\
\hline$U_{1223}$ & $0 .(2)$ & $-0.000014(5)$ & $-0.00003(3)$ & $-0.000002(5)$ & $0.00022(6)$ & $-0.000056(6)$ & $0.000027(5)$ & $0.00037(5)$ & $-0.0000039(9)$ & $-0.00002(1)$ \\
\hline$U_{1233}$ & $0 .(1)$ & $-0.00000(1)$ & $-0.000005(1)$ & $-0.000002(2)$ & $-0.00000(1)$ & $0.0005(2)$ & $-0.000001(2)$ & $-0.00001(2)$ & $-0.000003(2)$ & $-0.00002(2)$ \\
\hline$U_{1333}$ & $0 .(2)$ & $-0.00005(2)$ & $-0.000014(5)$ & $-0.000017(1)$ & $-0.000025(3)$ & $0.00022(6)$ & $-0.0000061(7)$ & $-0.000010(2)$ & $-0.000019(6)$ & $0.00006(2)$ \\
\hline$U_{2222}$ & $0.0(2)$ & $0.000001(0)$ & $-0.00001(1)$ & $-0.0021(2)$ & $-0.0008(2)$ & $-0.00000(2)$ & $0.0010(2)$ & $0.0005(2)$ & $-0.0000045(5)$ & $0.000003(1)$ \\
\hline$U_{2223}$ & $0 .(2)$ & $-0.000002(1)$ & $-0.00005(2)$ & $0.00002(3)$ & $0.0001(1)$ & $-0.000025(3)$ & $-0.00002(3)$ & $-0.0002(1)$ & $-0.0000009(4)$ & $-0.000003(2)$ \\
\hline$U_{2233}$ & $0 .(2)$ & $-0.000004(3)$ & $-0.000002(1)$ & $-0.000035(5)$ & $-0.00004(3)$ & $0.0001(1)$ & $0.000009(5)$ & $-0.00011(2)$ & $-0.0000054(5)$ & $0.000020(3)$ \\
\hline$U_{2333}$ & $0.0(2)$ & $-0.000002(6)$ & $-0.000004(3)$ & $-0.000004(2)$ & $-0.000006(8)$ & $-0.00004(3)$ & $0.000000(2)$ & $0.000006(7)$ & $-0.000002(1)$ & $-0.000004(5)$ \\
\hline$U_{3333}$ & $0 .(2)$ & $-0.000008(9)$ & $-0.000002(6)$ & $-0.000027(1)$ & $-0.000009(1)$ & $-0.000006(8)$ & $-0.000006(1)$ & $-0.000002(1)$ & $-0.000035(5)$ & $0.000048(5)$ \\
\hline
\end{tabular}

Table 22: Anharmonic Gram-Charlier coefficients obtained in 3rd and 4th order anharmonic thermal motion refinement (no intensity cut-off). Values higher than three standard uncertainties highlighted with cyan.

\begin{tabular}{|c|c|c|c|c|c|c|c|c|c|c|}
\hline & \multicolumn{3}{|c|}{ PT-11(S-Ph) } & \multicolumn{3}{|c|}{$\mathrm{PT}-2(\mathrm{Cl})$} & \multicolumn{2}{|c|}{ PT-8(Br) } & \multicolumn{2}{|c|}{ PT-10(I) } \\
\hline & HAR & MM & $\mathrm{MM}($ freeXH) & HAR & MM & $\mathrm{MM}($ freeXH $)$ & HAR & $\mathrm{MM}$ & HAR & MM \\
\hline$U_{111}$ & $0.00(3)$ & $-0.00002(2)$ & $-0.00002(1)$ & $0.000019(7)$ & $0.00003(6)$ & $0.00003(9)$ & $-0.000001(6)$ & $0.00001(7)$ & $-0.0010(1)$ & $-0.0009(1)$ \\
\hline$U_{112}$ & $0.0(2)$ & $-0.00004(1)$ & $-0.00004(1)$ & $0.00005(1)$ & $0.00011(3)$ & $0.0001(4)$ & $0.000038(9)$ & $0.0001(4)$ & $0.00013(1)$ & $0.00051(5)$ \\
\hline$U_{113}$ & $0.0(2)$ & $-0.00002(4)$ & $-0.00002(4)$ & $0.000016(4)$ & $0.000052(8)$ & $0.00006(9)$ & $0.000007(3)$ & $-0.0002(9)$ & $-0.00005(3)$ & $-0.00009(1)$ \\
\hline$U_{122}$ & $0.0(2)$ & $0.0002(3)$ & $0.0002(3)$ & $0.00004(3)$ & $0.000035(9)$ & $0.000035(1)$ & $-0.00003(2)$ & $0.000004(9)$ & $-0.000028(3)$ & $0.0002(8)$ \\
\hline$U_{123}$ & $0.0(2)$ & $0.00003(3)$ & $0.00003(3)$ & $0.000005(3)$ & $-0.00025(5)$ & $-0.00025(6)$ & $-0.000004(2)$ & $-0.0004(6)$ & $-0.00001(1)$ & $0.000008(4)$ \\
\hline$U_{133}$ & $0.00(3)$ & $0.00005(1)$ & $0.00005(1)$ & $0.000019(6)$ & $-0.000041(3)$ & $-0.00004(3)$ & $0.000013(5)$ & $-0.00003(3)$ & $0.000026(4)$ & $0.00013(7)$ \\
\hline$U_{222}$ & $0.00(1)$ & $0.000005(1)$ & $0.000005(1)$ & $0.0002(1)$ & $0.00014(9)$ & $0.00015(1)$ & $0.0000(1)$ & $-0.0001(2)$ & $0.000042(1)$ & $0.00005(1)$ \\
\hline$U_{223}$ & $0.0(1)$ & $0.00004(2)$ & $0.00004(2)$ & $-0.00012(2)$ & $-0.00002(6)$ & $-0.00002(7)$ & $-0.00016(2)$ & $-0.00002(6)$ & $-0.000001(1)$ & $0.000009(3)$ \\
\hline$U_{233}$ & $0.0(1)$ & $0.000004(7)$ & $0.000004(7)$ & $-0.000013(6)$ & $-0.000061(4)$ & $-0.00006(2)$ & $-0.000014(5)$ & $-0.00006(2)$ & $0.000014(2)$ & $0.00004(4)$ \\
\hline$U_{333}$ & $0.00(2)$ & $0.00007(1)$ & $0.00007(1)$ & $-0.000016(3)$ & $-0.0000088(2)$ & $-0.000009(3)$ & $-0.000010(3)$ & $-0.000003(2)$ & $0.000058(7)$ & $0.00007(7)$ \\
\hline$U_{1111}$ & $0.00(9)$ & $0.00001(2)$ & $0.00002(2)$ & $-0.000060(3)$ & $-0.00004(2)$ & $-0.00004(5)$ & $-0.000025(3)$ & $-0.00005(3)$ & $-0.0023(2)$ & $0.0001(2)$ \\
\hline$U_{11}$ & $0 .(2)$ & $0.000004(1)$ & $0.000004(2)$ & $0.000000(4)$ & $0.000025(2)$ & $0.000025(2)$ & $-0.000017(4)$ & $-0.0001(2)$ & $-0.00005(2)$ & $-0.00013(5)$ \\
\hline$U_{111}$ & $0 .(2)$ & $-0.00003(3)$ & $-0.00003(5)$ & $-0.000026(2)$ & $-0.00007(4)$ & $-0.00007(7)$ & $-0.000006(1)$ & -0.00 & $-0.00014(4)$ & $0.0003(9)$ \\
\hline$U_{1122}$ & $0.00(8)$ & -0.0000142 & $-0.00001(1)$ & $-0.000068(8)$ & $0.0002(5)$ & $0.00019(6)$ & $-0.000052(7)$ & $0.0003(5)$ & $-0.000022(3)$ & $-0.000020(4)$ \\
\hline$U_{1123}$ & 0.(1) & $-0.000002(1)$ & $-0.000002(1)$ & $-0.000002(2)$ & $0.000086(7)$ & $0.00009(9)$ & $-0.000003(2)$ & & $0.000000(5)$ & $-0.000003(2)$ \\
\hline$U_{1133}$ & $0.0(2)$ & $-0.000002(1)$ & $-0.000002(2)$ & $-0.000022(1)$ & $-0.000002(2)$ & $-0.000001(2)$ & $-0.0000064(7)$ & $-0.00002(2)$ & $-0.00010(1)$ & $-0.00002(1)$ \\
\hline$U_{1222}$ & $0 .(1)$ & $-0.000001(5)$ & $-0.000001(2)$ & $0.00006(3)$ & $0.00013(5)$ & & $0.00004(4)$ & $-0.0005(5)$ & $-0.000002(1)$ & $0.0001(1)$ \\
\hline$U_{1223}$ & $0 .(2)$ & $-0.000004(4)$ & $-0.000004(4)$ & $-0.000003(5)$ & $-0.000031(2)$ & $-0.00003(3)$ & $0.000024(4)$ & $-0.0002(2)$ & $-0.0000038(9)$ & $0.000010(3)$ \\
\hline$U_{1233}$ & $0 .(1)$ & $-0.000004(6)$ & $-0.000004(6)$ & $-0.000002(2)$ & $-0.000005(5)$ & $-0.000005(7)$ & $-0.000003(2)$ & $0.000001(6)$ & $-0.000002(2)$ & $-0.000005(5)$ \\
\hline$U_{1333}$ & $0 .(1)$ & $-0.00003(4)$ & $-0.00003(1)$ & $-0.0000169(9)$ & $-0.000057(4)$ & $-0.00006(6)$ & $-0.0000061(7)$ & $-0.00005(4)$ & $-0.000019(6)$ & $0.0003(5)$ \\
\hline$U_{2222}$ & $0.0(2)$ & $0.0000004(0)$ & $0.0000004(1)$ & $-0.0021(1)$ & $-0.00096(8)$ & & $0.0008(2)$ & & $-0.0000045(5)$ & $0.000001(2)$ \\
\hline$U_{222}$ & $0 .(2)$ & $-0.00001(2)$ & $-0.00001(4)$ & $0.00002(2)$ & $-0.000001(2)$ & $-0.0000004(3)$ & $-0.00002(3)$ & $-0.00001(3)$ & $-0.0000009(4)$ & $0.000001(2)$ \\
\hline$U_{2233}$ & $0 .(2)$ & $-0.00005(2)$ & $-0.00005(2)$ & $-0.000035(5)$ & $-0.000025(2)$ & $-0.000025(3)$ & $0.000007(4)$ & $-0.00001(2)$ & $-0.0000054(5)$ & $0.00004(2)$ \\
\hline$U_{2333}$ & $0.0(2)$ & $-0.000004(1)$ & $-0.000004(6)$ & $-0.000004(2)$ & $0.0004(1)$ & $0.0004(1)$ & $-0.000001(2)$ & $0.0006(2)$ & $-0.000002(1)$ & $-0.00001(7)$ \\
\hline$U_{3333}$ & $0 .(2)$ & $-0.00001(1)$ & $-0.00001(1)$ & $-0.000027(1)$ & $-0.000009(0)$ & $-0.000009(1)$ & $-0.0000063(9)$ & $-0.000004(1)$ & $-0.000035(5)$ & $0.00003(1)$ \\
\hline
\end{tabular}




\section{Energy of interactions}

Table 23: Columns 1-4: RMSD (units: $\mathrm{kJ} / \mathrm{mol}$ ) between the dimer interaction energies obtained by the supramolecular approach for experimental and optimised crystal geometries. Columns 5-8: RMSD divided by mean absolute value of dimer interaction energy calculated for the optimised structures. The results are presented in the format: (with intensity cutoff / no intensity cutoff).

\begin{tabular}{|c|c|c|c|c|c|c|c|c|c|}
\hline & \multirow[b]{2}{*}{ Structure } & \multicolumn{4}{|c|}{ RMSD $[\mathrm{kJ} / \mathrm{mol}]$} & \multicolumn{4}{|c|}{ RMSD $/$ mean $(\| \mathrm{E}($ optimised $) \|)[\%]$} \\
\hline & & $\mathrm{PT}-2(\mathrm{Cl})$ & PT-8(Br) & PT-10(I) & $\mathrm{PT}-11(\mathrm{~S}-\mathrm{Ph})$ & $\mathrm{PT}-2(\mathrm{Cl})$ & $\mathrm{PT}-8(\mathrm{Br})$ & PT-10(I) & $\mathrm{PT}-11(\mathrm{~S}-\mathrm{Ph})$ \\
\hline \multirow[t]{5}{*}{ harmonic } & $\mathrm{HAR}_{\text {anis }}$ & $0.64 / 0.63$ & $0.86 / 0.96$ & $1.90 / 0.94$ & $0.36 / 0.39$ & $3.7 \% / 3.6 \%$ & $5.3 \% / 5.9 \%$ & $11.0 \% / 5.4 \%$ & $1.7 \% / 1.9 \%$ \\
\hline & $\mathrm{HAR}_{\text {iso }}$ & $0.55 / 0.50$ & $0.96 / 1.03$ & $1.62 / 0.90$ & $0.32 / 0.35$ & $3.2 \% / 2.9 \%$ & $5.9 \% / 6.3 \%$ & $9.4 \% / 5.2 \%$ & $1.6 \% / 1.7 \%$ \\
\hline & IAM & 8.38 & 7.30 & 2.26 & 4.56 & $48.8 \%$ & $44.9 \%$ & $13.2 \%$ & $22.2 \%$ \\
\hline & $\mathrm{MM}$ & $0.36 / 0.38$ & $1.03 / 1.09$ & $1.32 / 1.12$ & $0.28 / 0.34$ & $2.1 \% / 2.2 \%$ & $6.3 \% / 6.7 \%$ & $7.7 \% / 6.5 \%$ & $1.4 \% / 1.7 \%$ \\
\hline & $\mathrm{MM}($ freeXH) & $1.60 / 0.82$ & & & $1.70 / 1.46$ & $9.3 \% / 4.8 \%$ & & & $8.3 \% / 7.1 \%$ \\
\hline \multirow[t]{3}{*}{ anharmonic $(\mathrm{n}=3)$} & $\mathrm{HAR}_{\text {anis }}$ & $0.74 / 0.69$ & $0.87 / 0.89$ & $1.78 / 0.90$ & $0.40 / 0.41$ & $4.3 \% / 4.0 \%$ & $5.3 \% / 5.5 \%$ & $10.3 \% / 5.2 \%$ & $1.9 \% / 2.0 \%$ \\
\hline & MM & $0.36 / 0.38$ & $1.07 / 0.84$ & $1.32 / 1.10$ & $0.32 / 0.33$ & $2.1 \% / 2.2 \%$ & $6.6 \% / 5.2 \%$ & $7.7 \% / 6.4 \%$ & $1.5 \% / 1.6 \%$ \\
\hline & $\mathrm{MM}($ freeXH) & $1.68 / 0.37$ & & & $1.61 / 1.29$ & $9.8 \% / 2.1 \%$ & & & $7.9 \% / 6.3 \%$ \\
\hline \multirow[t]{3}{*}{ anharmonic $(\mathrm{n}=3,4)$} & $\mathrm{HAR}_{\text {anis }}$ & $0.37 / 0.38$ & $0.85 / 0.89$ & $1.83 / 1.37$ & $0.42 / 0.45$ & $2.1 \% / 2.2 \%$ & $5.2 \% / 5.5 \%$ & $10.7 \% / 7.9 \%$ & $2.0 \% / 2.2 \%$ \\
\hline & MM & $0.37 / 0.39$ & $1.03 / 1.00$ & $1.34 / 1.11$ & $0.32 / 0.33$ & $2.1 \% / 2.3 \%$ & $6.3 \% / 6.1 \%$ & $7.8 \% / 6.4 \%$ & $1.6 \% / 1.6 \%$ \\
\hline & $\mathrm{MM}($ freeXH) & $1.69 / 0.64$ & & & $1.61 / 1.38$ & $9.8 \% / 3.7 \%$ & & & $7.9 \% / 6.7 \%$ \\
\hline
\end{tabular}

Table 24: Energies of interactions of selected strongly interacting dimers. Units: kJ/mol. The results are presented in the format: (with intensity cutoff / no intensity cutoff).

\begin{tabular}{|c|c|c|c|c|c|}
\hline & $\begin{array}{l}\text { Structure } \\
\text { Dimer }\end{array}$ & $\begin{array}{c}\mathrm{PT}-2(\mathrm{Cl}) \\
\text { dimer1 }\end{array}$ & $\begin{array}{l}\mathrm{PT}-8(\mathrm{Br}) \\
\text { dimer10 }\end{array}$ & $\begin{array}{c}\text { PT-10(I) } \\
\text { dimer2 }\end{array}$ & $\begin{array}{c}\mathrm{PT}-11(\mathrm{~S}-\mathrm{Ph}) \\
\quad \text { dimer1 }\end{array}$ \\
\hline harmonic & $\begin{array}{l}\text { optimised } \\
\text { HAR }_{\text {anis }} \\
\text { HAR }_{\text {iso }} \\
\text { IAM } \\
\text { MM } \\
\text { MM(freeXH) }\end{array}$ & $\begin{array}{r}-60.6 \\
-61.8 /-61.8 \\
-61.5 /-61.4 \\
-40.1 \\
-60.3 /-60.2 \\
-64.4 /-62.4\end{array}$ & $\begin{array}{r}-55.7 \\
-54.1 /-53.7 \\
-53.8 /-53.5 \\
-37.9 \\
-53.4 /-53.6\end{array}$ & $\begin{array}{r}-32.2 \\
-34.3 /-34.3 \\
-34.1 /-34.2 \\
-35.1 \\
-33.6 /-33.4\end{array}$ & $\begin{array}{r}-52.8 \\
-53.6 /-53.6 \\
-53.5 /-53.5 \\
-38.6 \\
-52.4 /-52.2 \\
-47.5 /-48.6\end{array}$ \\
\hline anharmonic $(\mathrm{n}=3)$ & $\begin{array}{l}\text { HAR }_{\text {anis }} \\
\text { MM } \\
\text { MM(freeXH) }\end{array}$ & $\begin{array}{l}-62.1 /-62.0 \\
-60.3 /-60.2 \\
-64.6 /-60.2\end{array}$ & $\begin{array}{l}-54.0 /-53.9 \\
-53.3 /-54.0\end{array}$ & $\begin{array}{l}-34.4 /-34.4 \\
-33.6 /-33.4\end{array}$ & $\begin{array}{l}-53.8 /-53.7 \\
-52.2 /-52.3 \\
-47.8 /-49.1\end{array}$ \\
\hline anharmonic $(\mathrm{n}=3,4)$ & $\begin{array}{l}\text { HAR }_{\text {anis }} \\
\text { MM } \\
\text { MM(freeXH) }\end{array}$ & $\begin{array}{l}-60.6 /-60.7 \\
-60.3 /-60.1 \\
-64.6 /-61.9\end{array}$ & $\begin{array}{l}-54.1 /-54.0 \\
-53.4 /-54.0\end{array}$ & $\begin{array}{l}-34.4 /-34.5 \\
-33.6 /-33.3\end{array}$ & $\begin{array}{l}-53.8 /-53.9 \\
-52.2 /-52.3 \\
-47.8 /-48.8\end{array}$ \\
\hline
\end{tabular}

Table 25: Energies of halogen bonds. Units: $\mathrm{kJ} / \mathrm{mol}$. The results are presented in the format: (with intensity cutoff / no intensity cutoff).

\begin{tabular}{llrrr}
\hline \hline & Structure & PT-2(Cl) & PT-8(Br) & \multicolumn{1}{c}{ PT-10(I) } \\
\hline \multirow{3}{*}{ harmonic } & optimised & -4.9 & -7.1 & -35.9 \\
& HAR $_{\text {anis }}$ & $-4.7 /-4.7$ & $-6.8 /-6.8$ & $-32.3 /-32.4$ \\
& HAR $_{\text {iso }}$ & $-4.7 /-4.7$ & $-6.8 /-6.8$ & $-32.4 /-32.4$ \\
& IAM & -5.3 & -7.2 & -31.2 \\
& MM & $-4.7 /-4.7$ & $-6.8 /-6.8$ & $-33.0 /-33.0$ \\
anharmonic $(\mathrm{n}=3)$ & MM(freeXH) & $-4.5 /-4.5$ & & \\
& HAR & & \\
& MMis & $-4.7 /-4.7$ & $-6.8 /-6.8$ & $-32.2 /-32.2$ \\
& MM(freeXH) & $-4.7 /-4.7$ & $-6.7 /-6.8$ & $-33.0 /-33.1$ \\
anharmonic $(\mathrm{n}=3,4)$ & HAR & & \\
& MMis & $-4.7 /-4.7$ & $-6.8 /-6.8$ & $-32.0 /-32.0$ \\
& MM(freeXH) & $-4.7 /-4.7$ & $-6.8 /-6.8$ & $-32.9 /-33.0$ \\
\hline \hline
\end{tabular}


Table 26: Cohesive energies. Units: kJ/mol. The results are presented in the format: (with intensity cutoff / no intensity cutoff).

\begin{tabular}{|c|c|c|c|c|c|}
\hline & Structure & $\mathrm{PT}-2(\mathrm{Cl})$ & PT-8(Br) & PT-10(I) & $\mathrm{PT}-11(\mathrm{~S}-\mathrm{Ph})$ \\
\hline harmonic & $\begin{array}{l}\text { optimised } \\
\text { HAR }_{\text {anis }} \\
\text { HAR }_{\text {iso }} \\
\text { IAM } \\
\text { MM } \\
\text { MM(freeXH) }\end{array}$ & $\begin{array}{r}-97.2 \\
-93.1 /-93.1 \\
-92.9 /-93.0 \\
150.4 \\
-94.3 /-94.5 \\
-79.0 /-81.1\end{array}$ & $\begin{array}{r}-96.4 \\
-89.5 /-89.4 \\
-89.2 /-89.2 \\
172.9 \\
-95.9 /-96.5\end{array}$ & $\begin{array}{r}-111.8 \\
-42.2 /-41.8 \\
-40.7 /-40.2 \\
486.8 \\
-98.0 /-99.9\end{array}$ & $\begin{array}{r}-114.3 \\
-108.1 /-108.1 \\
-108.6 /-108.6 \\
281.1 \\
-110.9 /-110.9 \\
-92.9 /-100.9\end{array}$ \\
\hline anharmonic $(\mathrm{n}=3)$ & $\begin{array}{l}\mathrm{HAR}_{\text {anis }} \\
\text { MM } \\
\text { MM(freeXH) }\end{array}$ & $\begin{array}{l}-93.1 /-93.1 \\
-94.3 /-94.5 \\
-80.3 /-81.5\end{array}$ & $\begin{array}{l}-90.6 /-89.6 \\
-96.0 /-96.5\end{array}$ & $\begin{array}{r}-38.1 /-37.2 \\
-98.6 /-101.8\end{array}$ & $\begin{array}{r}-108.1 /-108.1 \\
-110.7 /-110.9 \\
-92.9 /-102.1\end{array}$ \\
\hline anharmonic $(\mathrm{n}=3,4)$ & $\begin{array}{l}\mathrm{HAR}_{\text {anis }} \\
\text { MM } \\
\mathrm{MM}(\text { freeXH) }\end{array}$ & $\begin{array}{l}-94.2 /-94.2 \\
-94.3 /-94.5 \\
-79.0 /-83.3\end{array}$ & $\begin{array}{l}-89.0 /-88.8 \\
-95.8 /-96.3\end{array}$ & $\begin{array}{r}-24.6 /-22.1 \\
-99.1 /-102.4\end{array}$ & $\begin{array}{l}-107.8 /-107.8 \\
-110.6 /-110.9 \\
-133.0 /-101.9\end{array}$ \\
\hline
\end{tabular}

Table 27: Geometrical relaxation energies. Units: kJ/mol. The results are presented in the format: (with intensity cutoff / no intensity cutoff).

\begin{tabular}{|c|c|c|c|c|c|}
\hline & Structure & $\mathrm{PT}-2(\mathrm{Cl})$ & PT-8(Br) & PT-10(I) & $\mathrm{PT}-11(\mathrm{~S}-\mathrm{Ph})$ \\
\hline harmonic & $\begin{array}{l}\text { optimised } \\
\text { HAR }_{\text {anis }} \\
\text { HAR }_{\text {iso }} \\
\text { IAM } \\
\text { MM } \\
\text { MM(freeXH) }\end{array}$ & $\begin{array}{r}-5.6 \\
-9.3 /-9.2 \\
-9.3 /-9.2 \\
-247.9 \\
-8.5 /-8.5 \\
-23.9 /-21.2\end{array}$ & $\begin{array}{r}-7.3 \\
-13.7 /-13.7 \\
-13.9 /-13.9 \\
-273.2 \\
-5.9 /-5.6\end{array}$ & $\begin{array}{r}-3.6 \\
-72.2 /-72.6 \\
-74.5 /-75.0 \\
-606.4 \\
-15.5 /-13.9\end{array}$ & $\begin{array}{r}-8.9 \\
-15.7 /-15.7 \\
-14.9 /-14.9 \\
-404.7 \\
-12.4 /-12.2 \\
-27.5 /-19.4\end{array}$ \\
\hline anharmonic $(\mathrm{n}=3)$ & $\begin{array}{l}\mathrm{HAR}_{\text {anis }} \\
\text { MM } \\
\mathrm{MM}(\text { freeXH) }\end{array}$ & $\begin{array}{r}-9.5 /-9.4 \\
-8.3 /-8.5 \\
-22.8 /-20.7\end{array}$ & $\begin{array}{r}-13.6 /-13.6 \\
-5.8 /-5.7\end{array}$ & $\begin{array}{l}-75.8 /-77.0 \\
-14.6 /-11.6\end{array}$ & $\begin{array}{l}-15.7 /-15.7 \\
-12.6 /-12.2 \\
-27.6 /-18.4\end{array}$ \\
\hline anharmonic $(\mathrm{n}=3,4)$ & $\begin{array}{l}\text { HAR }_{\text {anis }} \\
\text { MM } \\
\text { MM(freeXH) }\end{array}$ & $\begin{array}{r}-8.0 /-7.9 \\
-8.4 /-8.5 \\
-21.8 /-18.8\end{array}$ & $\begin{array}{r}-14.5 /-14.6 \\
-6.0 /-5.9\end{array}$ & $\begin{array}{l}-89.7 /-92.2 \\
-14.0 /-11.0\end{array}$ & $\begin{array}{r}-16.15 /-16.12 \\
-12.7 /-12.2 \\
-27.9 /-18.5\end{array}$ \\
\hline
\end{tabular}




\section{$3 \quad$ Fractal dimension plots}

PT11, HAR, harmonic, $|F| \geq 2 \sigma(|F|)$
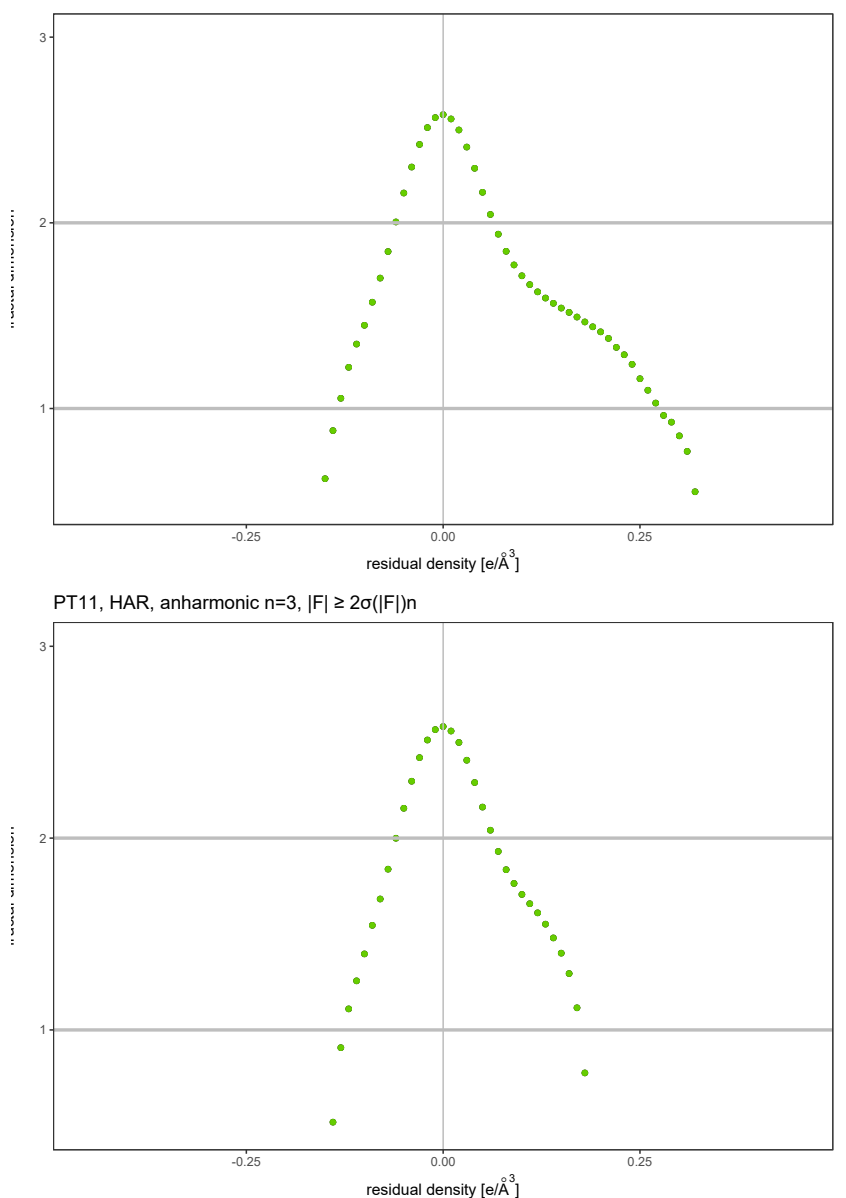

PT11, HAR, anharmonic $n=3,4,|F| \geq 2 \sigma(|F|)$

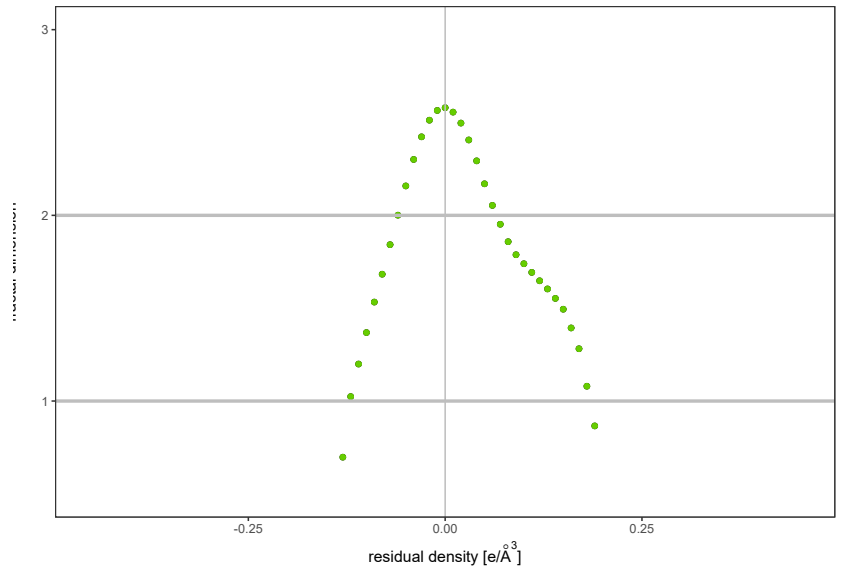

PT11, HAR, harmonic, no intensity cutoff

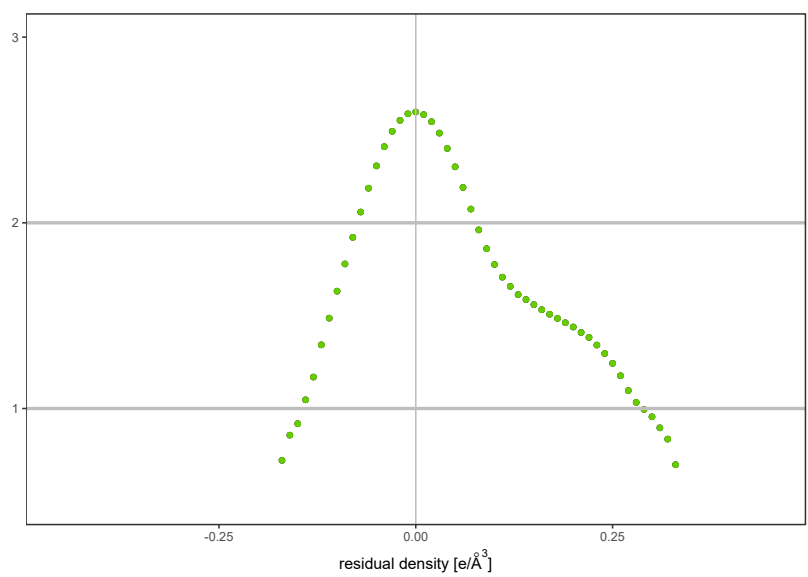

PT11, HAR, anharmonic $n=3$, no intensity cutoff

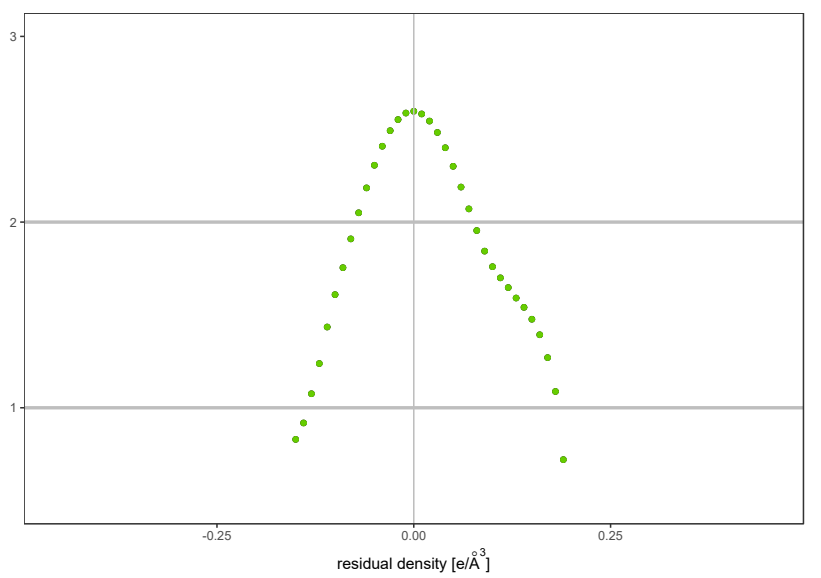

PT11, HAR, anharmonic $n=3,4$, no intensity cutoff

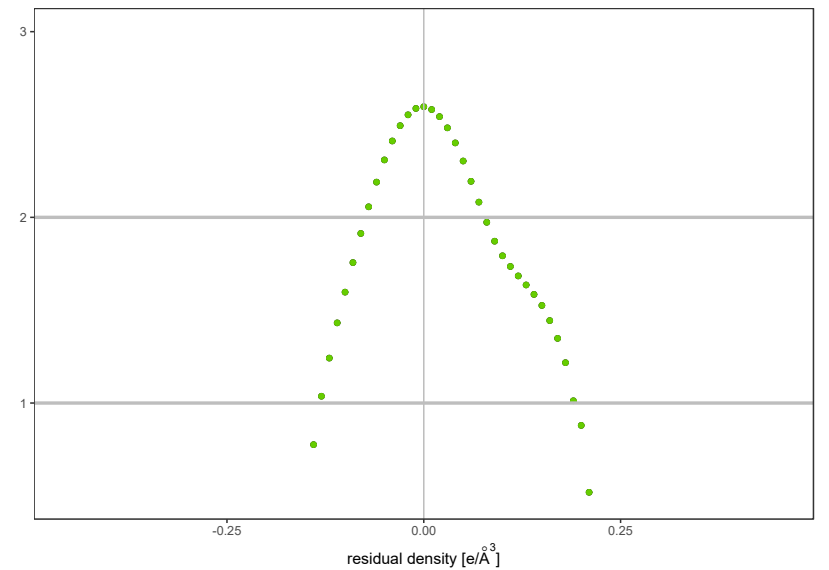

Figure 1: Fractal dimension plots for PT-11, HAR, left: $|F| \geq 2 \sigma(|F|)$ and right: all reflections included. 
PT11, XWR, harmonic, $|F| \geq 2 \sigma(|F|)$

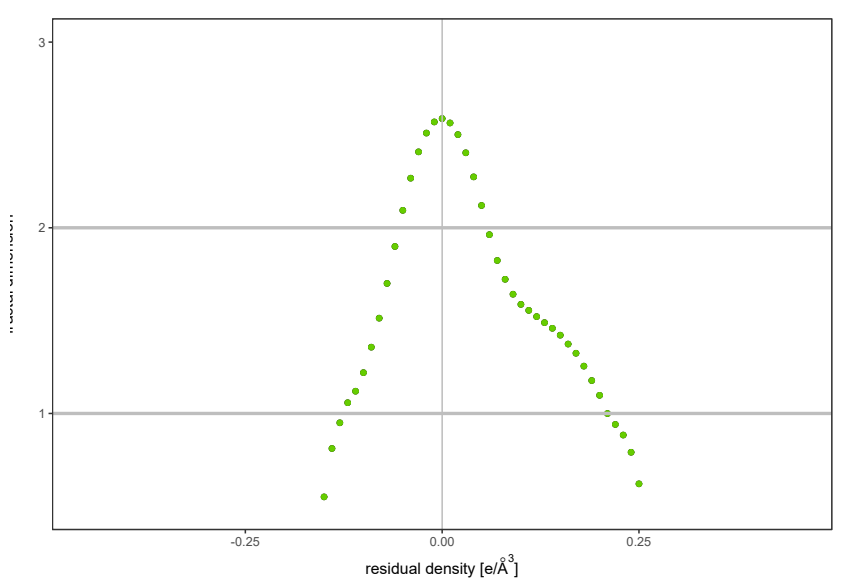

PT11, XWR, anharmonic $n=3,4,|F| \geq 2 \sigma(|F|)$

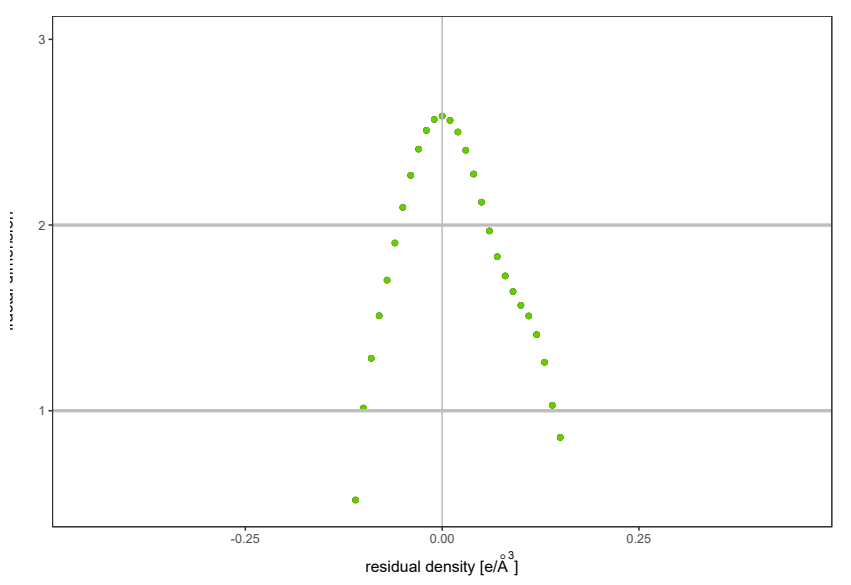

PT11, XWR, harmonic, no intensity cutoff

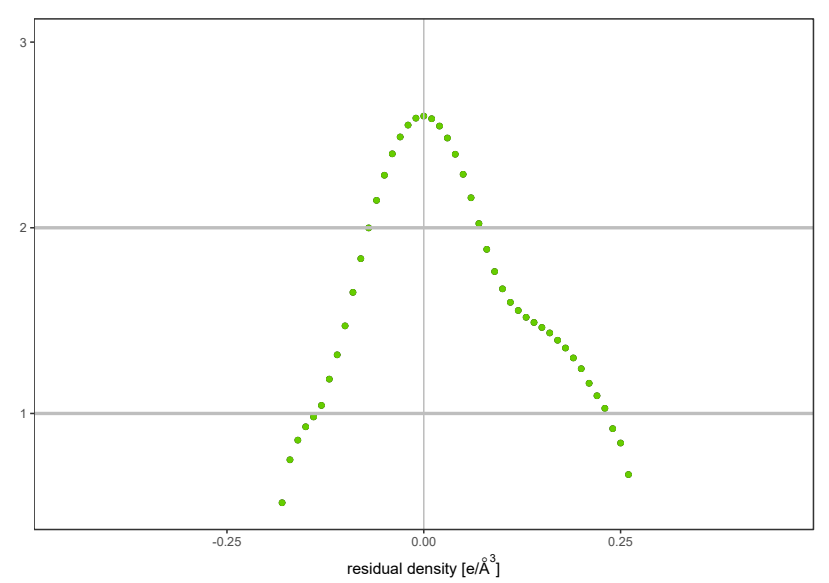

PT11, XWR, anharmonic $n=3,4$, no intensity cutoff

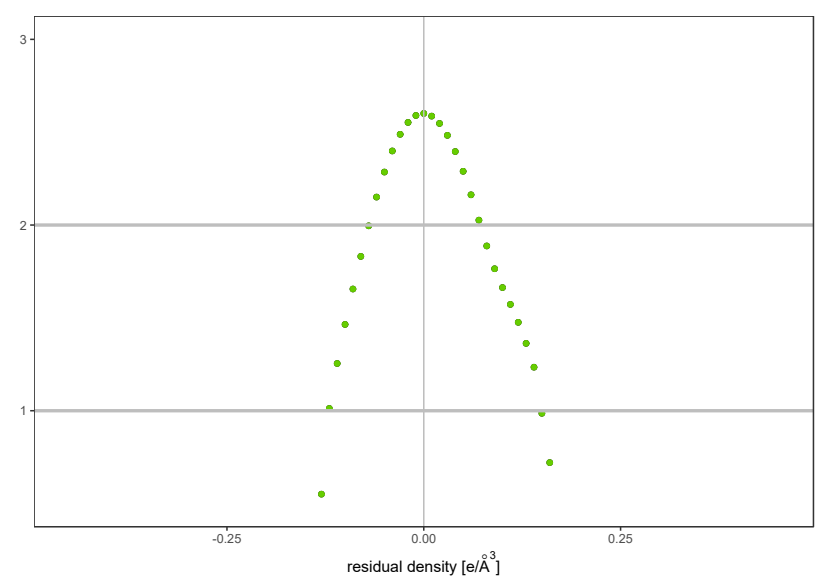

Figure 2: Fractal dimension plots for PT-11, XWR, left: $|F| \geq 2 \sigma(|F|)$ and right: all reflections included. 
PT11, MM, harmonic, $H$ positions restrained, $|\mathrm{F}| \geq 2 \sigma(|\mathrm{F}|)$

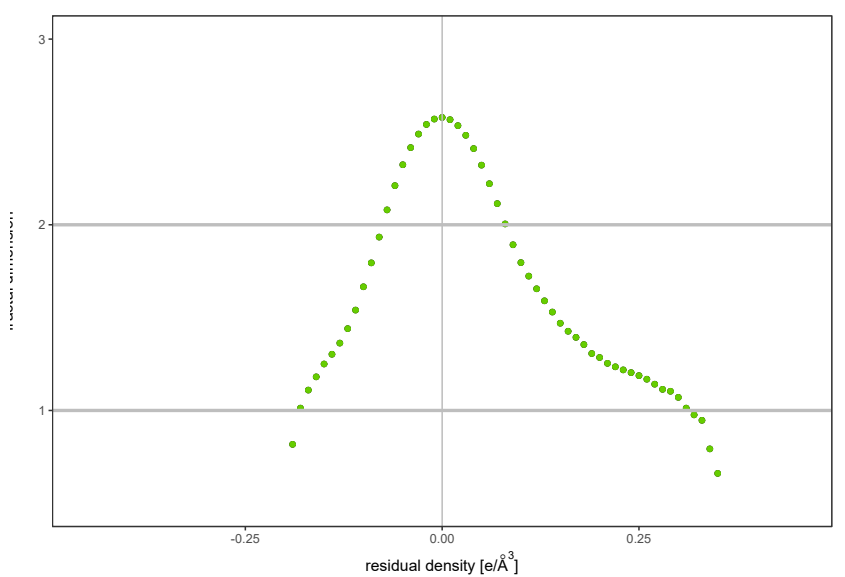

PT11, MM, anharmonic $n=3, H$ positions restrained, $|F| \geq 2 \sigma(|F|)$

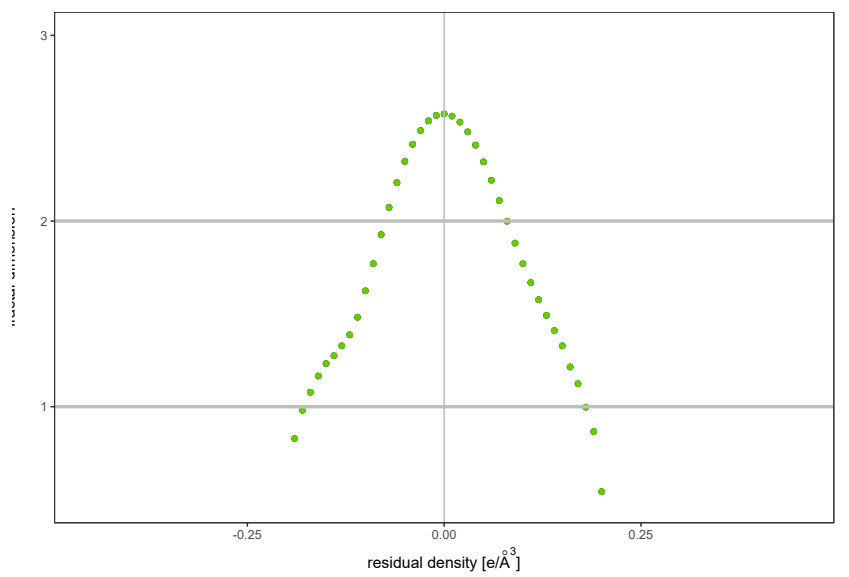

PT11, MM, anharmonic $n=3,4, H$ positions restrained, $|F| \geq 2 \sigma(|F|)$

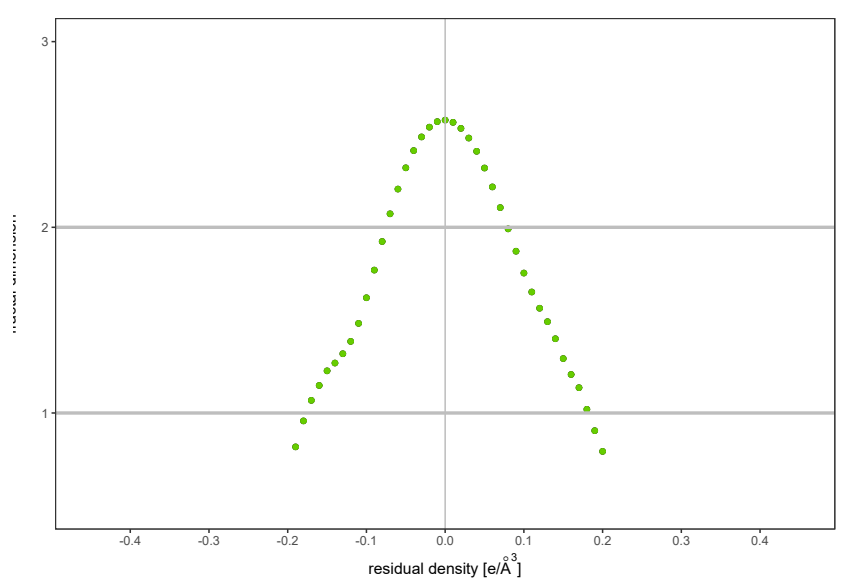

PT11, MM, harmonic, $\mathrm{H}$ positions restrained, no intensity cutoff

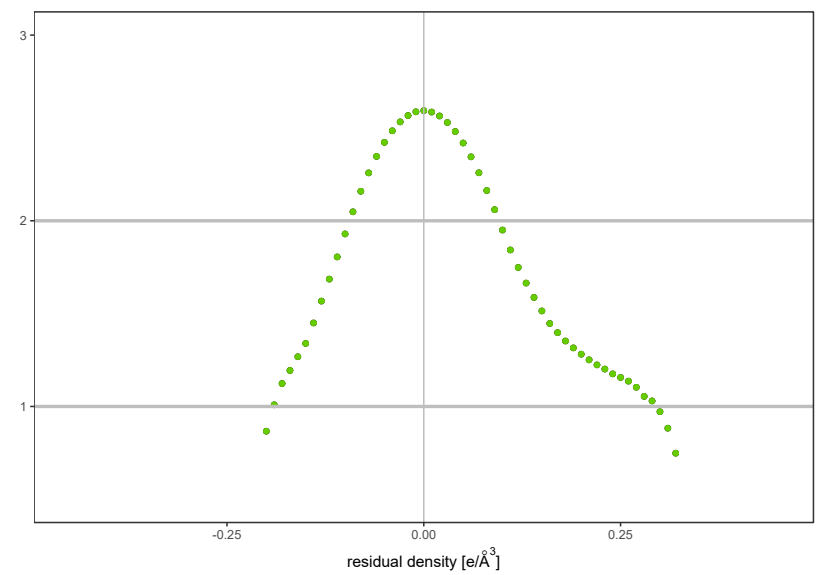

PT11, MM, anharmonic $n=3, H$ positions restrained, no intensity cutoff

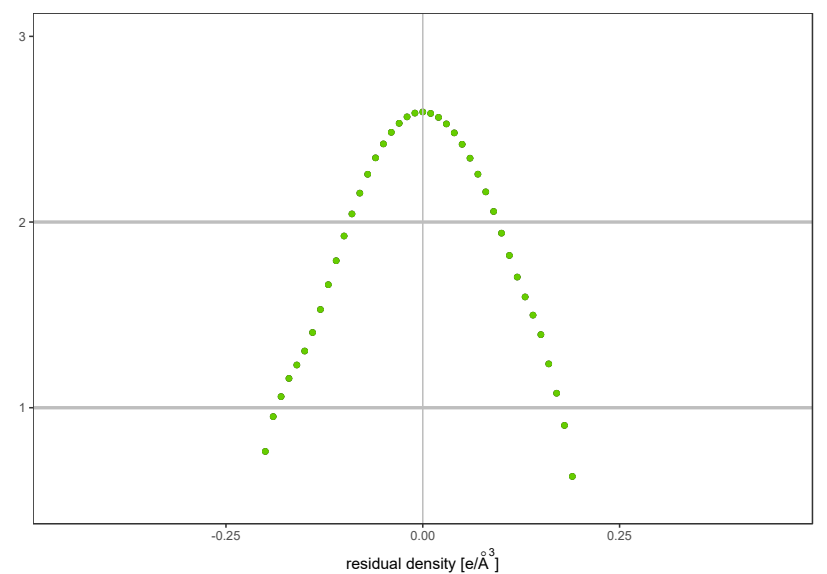

PT11, MM, anharmonic $n=3,4, H$ positions restrained, no intensity cutoff

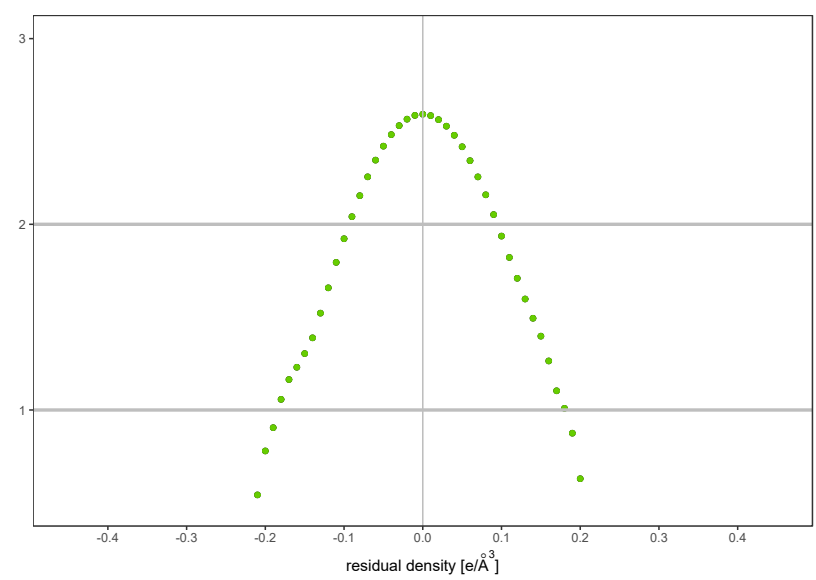

Figure 3: Fractal dimension plots for PT-11, MM, restrained X-H distances, left: $|F| \geq 2 \sigma(|F|)$ and right: all reflections included. 
PT11, MM, harmonic, $H$ positions unrestrained, $|F| \geq 2 \sigma(|F|)$

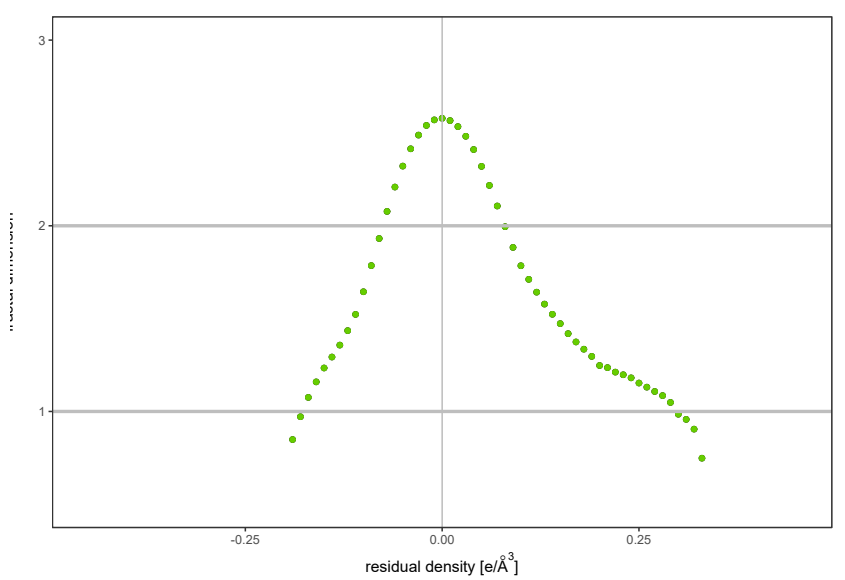

PT11, MM, anharmonic $n=3, H$ positions unrestrained, $|F| \geq 2 \sigma(|F|)$

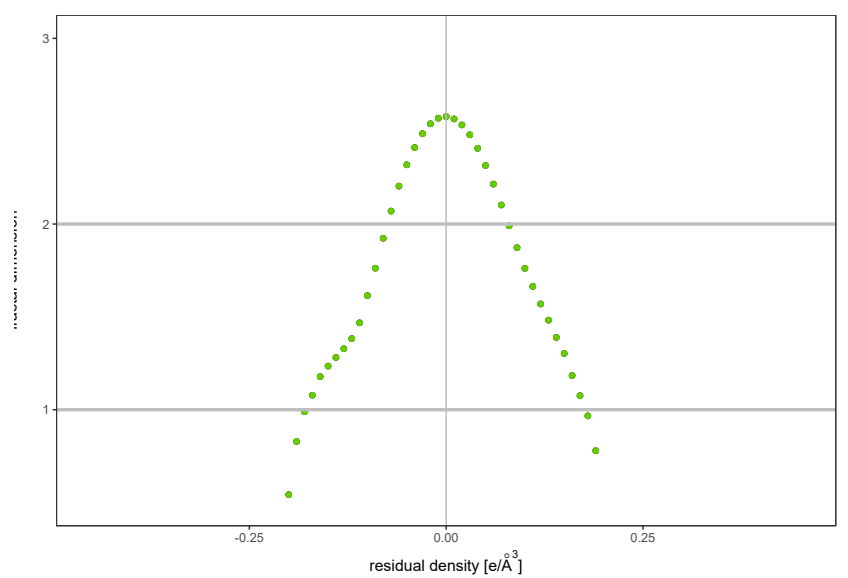

PT11, MM, anharmonic $n=3,4, H$ positions unrestrained, $|F| \geq 2 \sigma(|F|)$

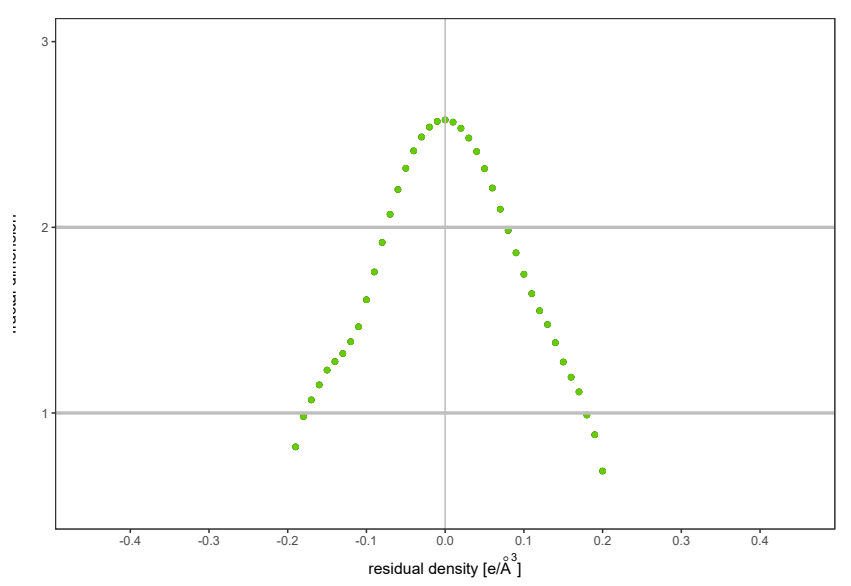

PT11, MM, harmonic, $\mathrm{H}$ positions unrestrained, no intensity cutoff

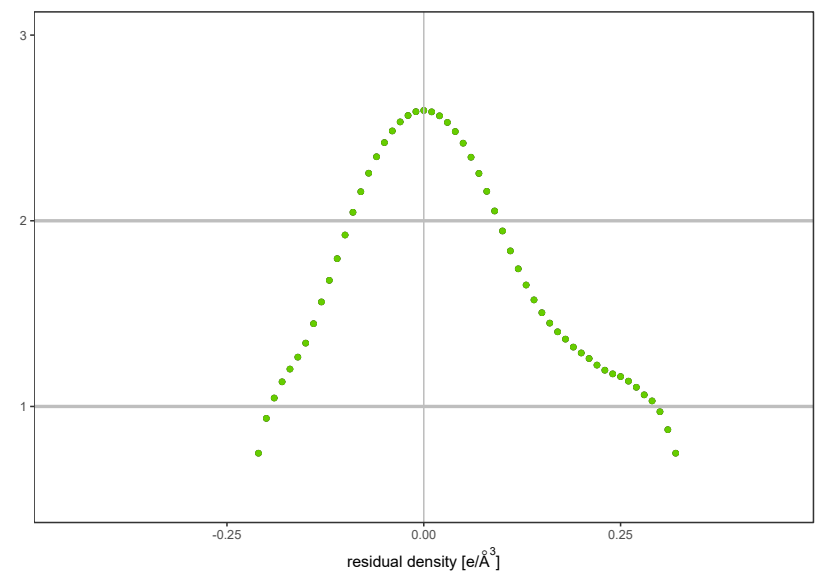

PT11, MM, anharmonic $n=3, H$ positions unrestrained, no intensity cutoff

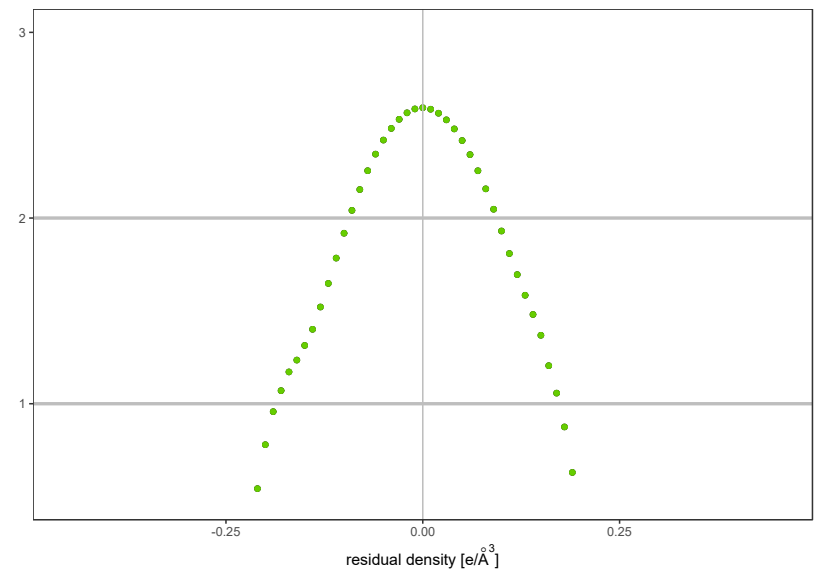

PT11, MM, anharmonic $n=3,4, H$ positions unrestrained, no intensity cutoff

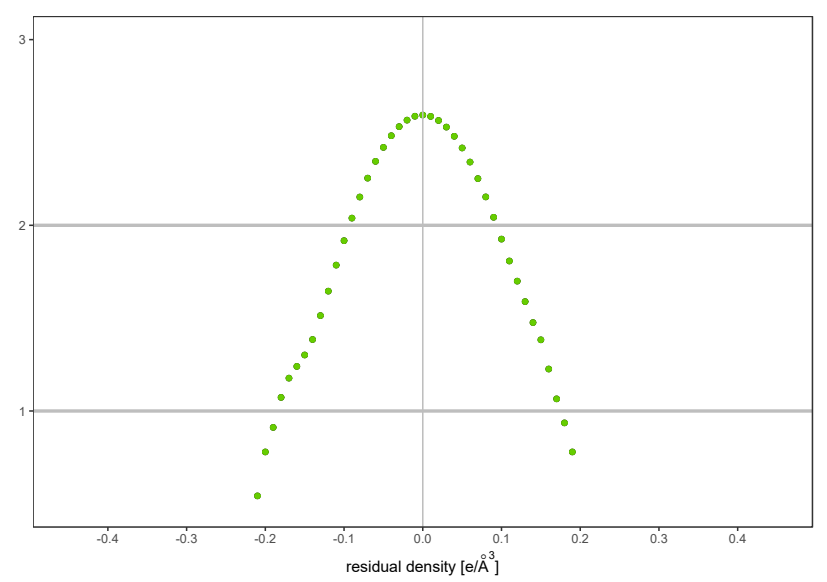

Figure 4: Fractal dimension plots for PT-11, MM, unrestrained X-H distances, left: $|F| \geq 2 \sigma(|F|)$ and right: all reflections included. 
PT2, HAR, harmonic, $|F| \geq 2 \sigma(|F|)$

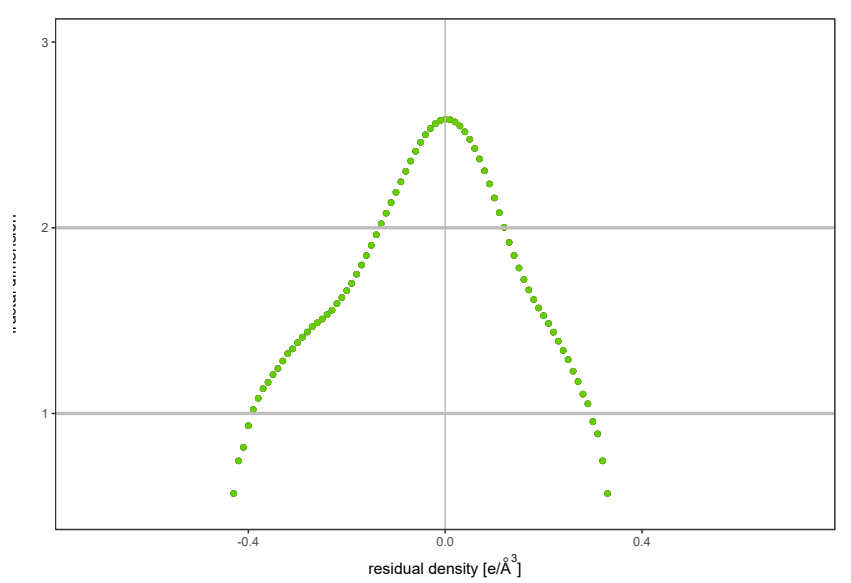

PT2, HAR, anharmonic $n=3,|F| \geq 2 \sigma(|F|) n$

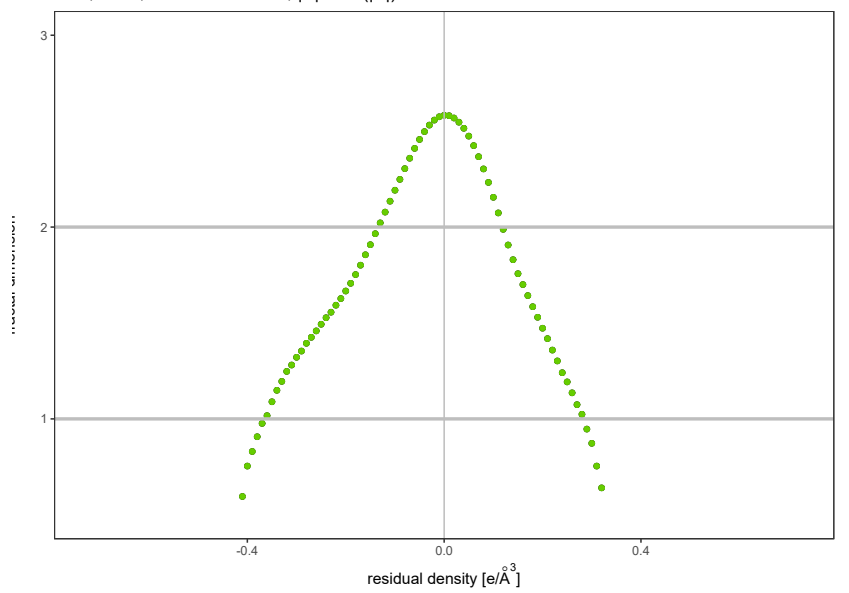

PT2, HAR, anharmonic $n=3,4,|F| \geq 2 \sigma(|F|)$

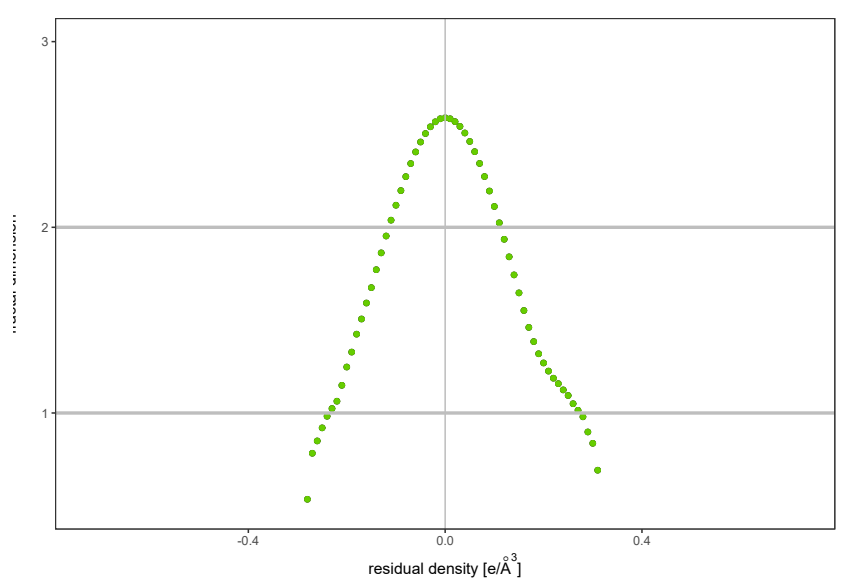

PT2, HAR, harmonic, no intensity cutoff

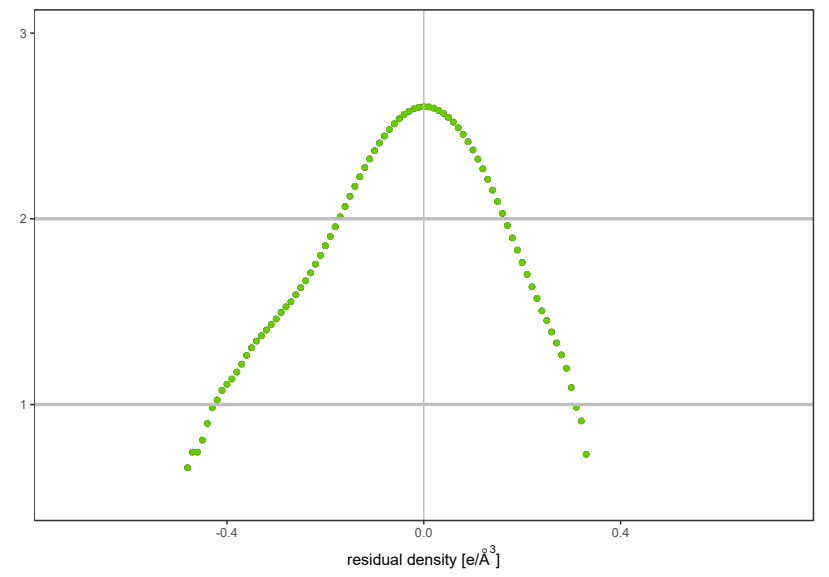

PT2, HAR, anharmonic n=3, no intensity cutoff

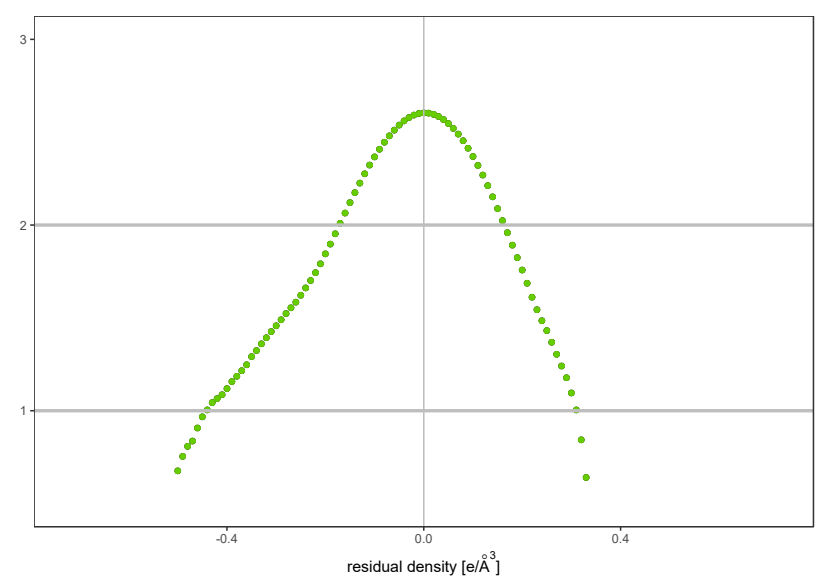

PT2, HAR, anharmonic $n=3,4$, no intensity cutoff

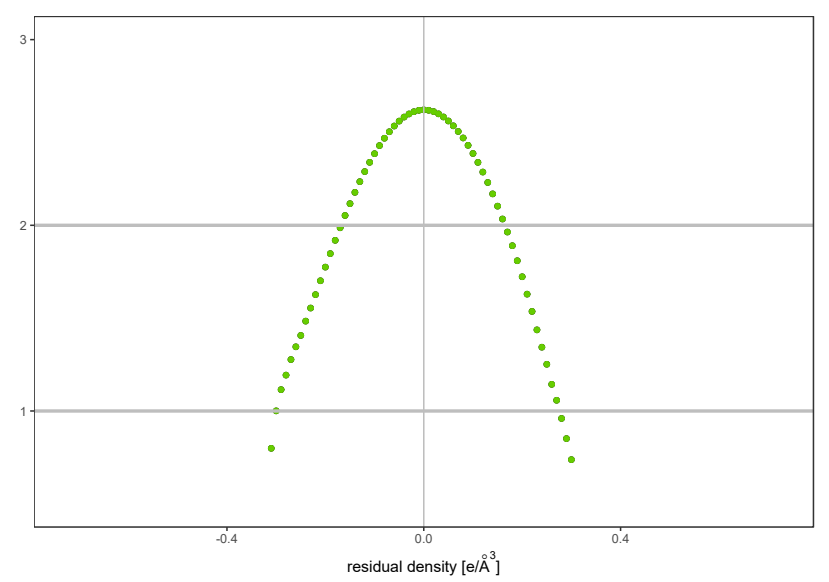

Figure 5: Fractal dimension plots for PT-2, HAR, left: $|F| \geq 2 \sigma(|F|)$ and right: all reflections included. 
PT2, XWR, harmonic, $|\mathrm{F}| \geq 2 \sigma(|\mathrm{F}|)$

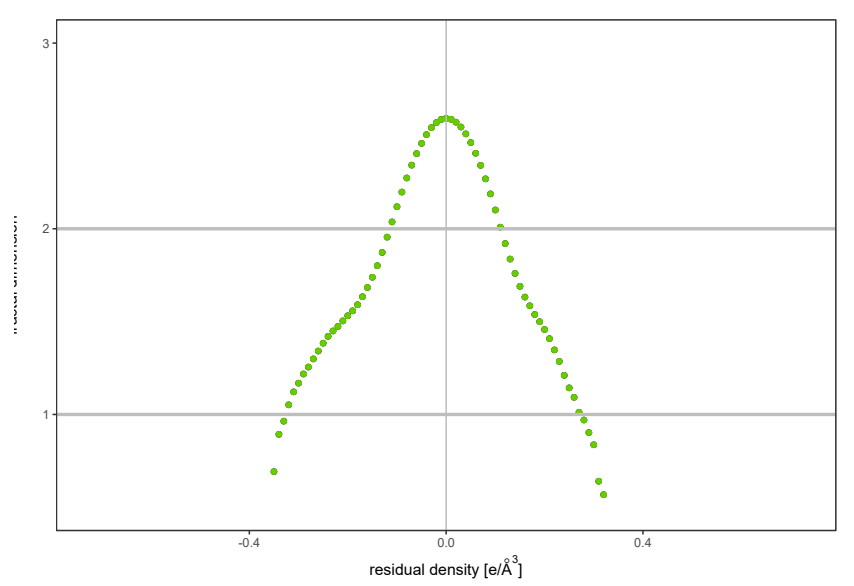

PT2, XWR, anharmonic $n=3,4,|F| \geq 2 \sigma(|F|)$

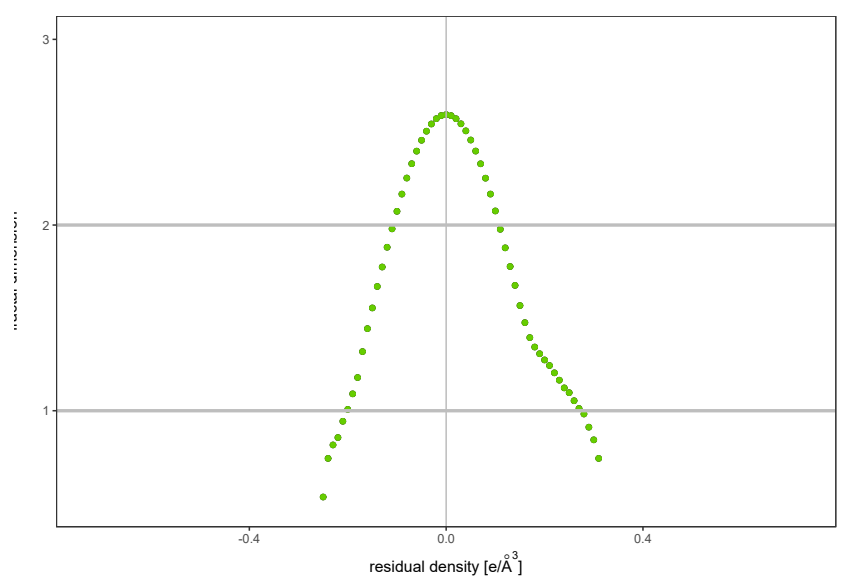

PT2, XWR, harmonic, no intensity cutoff

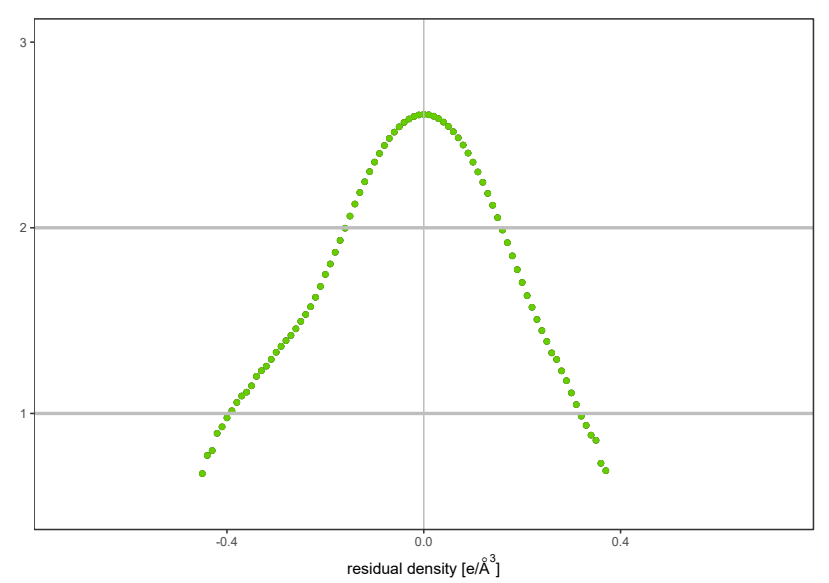

PT2, XWR, anharmonic $n=3,4$, no intensity cutoff

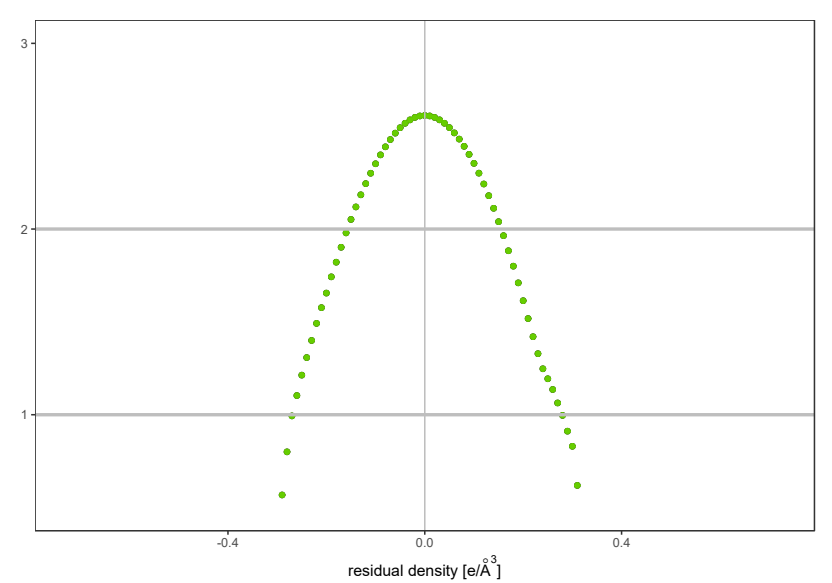

Figure 6: Fractal dimension plots for PT-2, XWR, left: $|F| \geq 2 \sigma(|F|)$ and right: all reflections included. 
PT2, MM, harmonic, $H$ positions restrained, $|F| \geq 2 \sigma(|F|)$

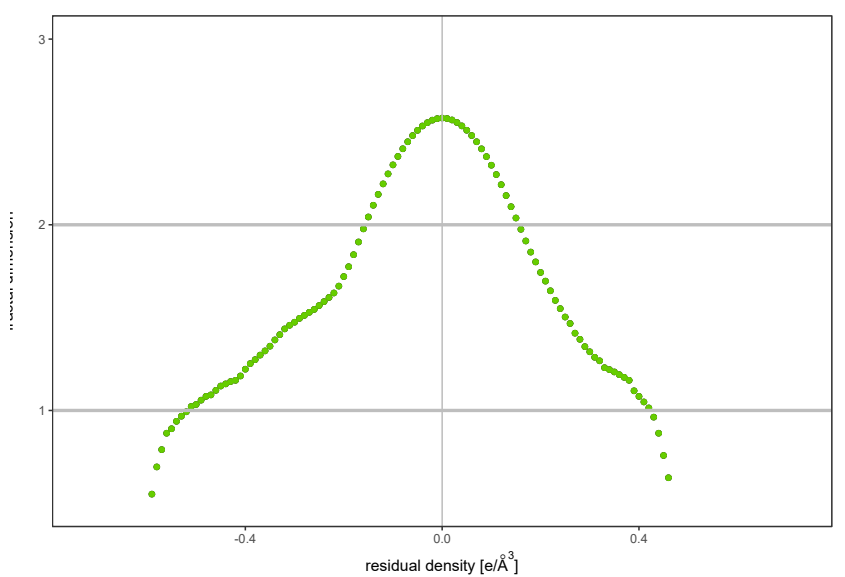

PT2, MM, anharmonic $n=3, H$ positions restrained, $|F| \geq 2 \sigma(|F|)$

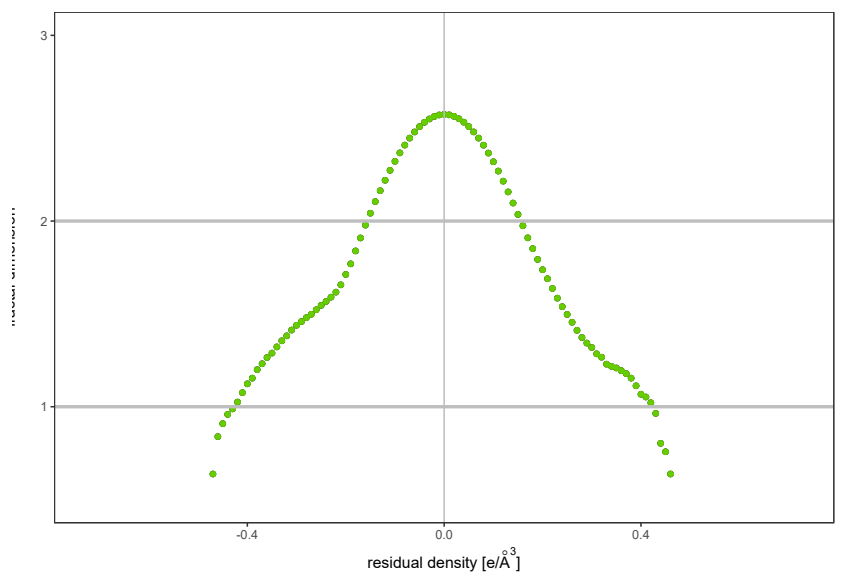

PT2, MM, anharmonic $n=3,4, H$ positions restrained, $|F| \geq 2 \sigma(|F|)$

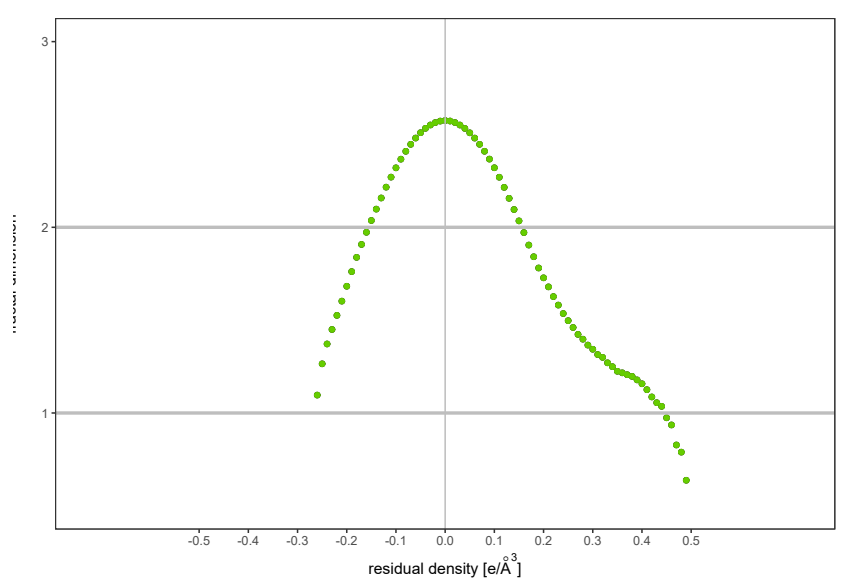

PT2, MM, harmonic, $\mathrm{H}$ positions restrained, no intensity cutoff

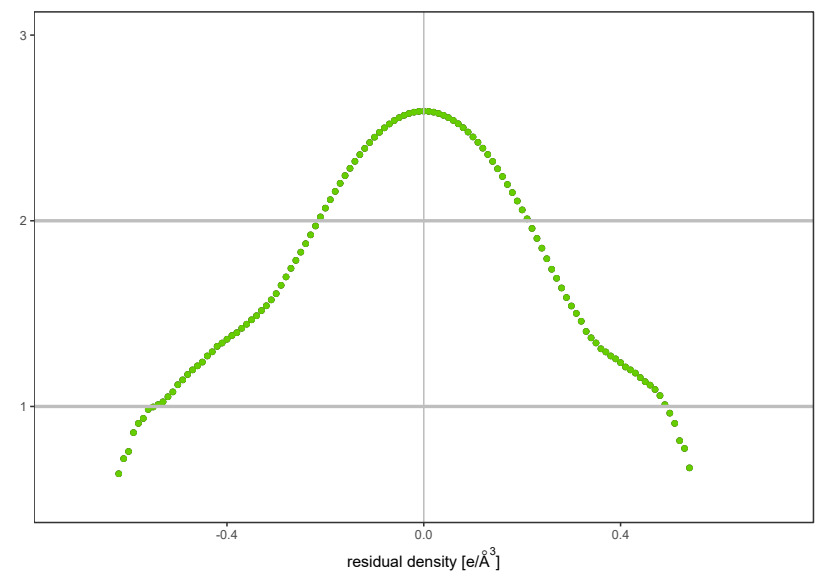

PT2, MM, anharmonic $n=3, H$ positions restrained, no intensity cutoff

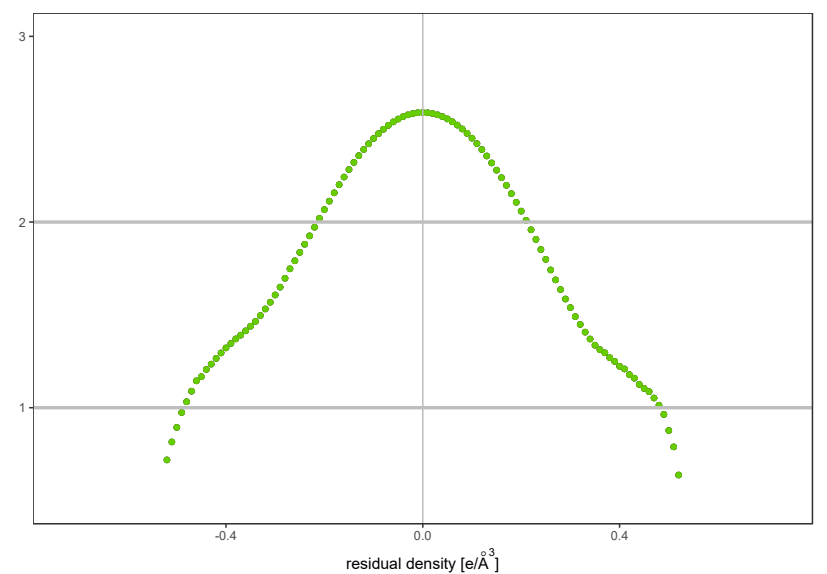

PT2, MM, anharmonic $n=3,4, H$ positions restrained, no intensity cutoff

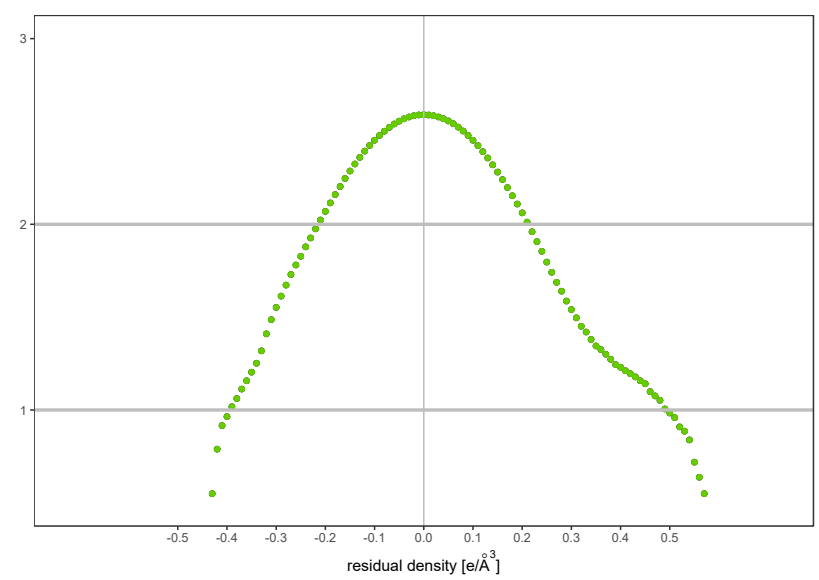

Figure 7: Fractal dimension plots for PT-2, MM, restrained X-H distances, left: $|F| \geq 2 \sigma(|F|)$ and right: all reflections included. 
PT2, MM, harmonic, $H$ positions unrestrained, $|F| \geq 2 \sigma(|F|)$

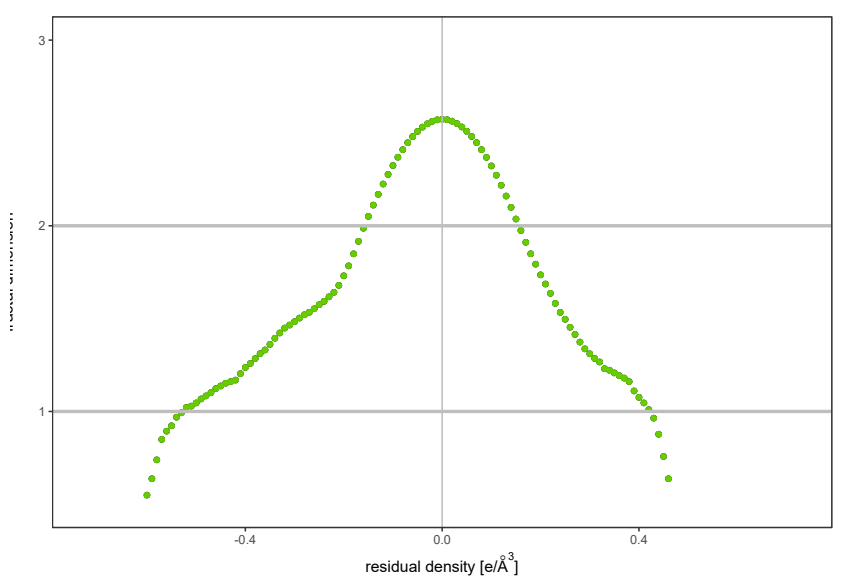

PT2, MM, anharmonic $n=3, H$ positions unrestrained, $|F| \geq 2 \sigma(|F|)$

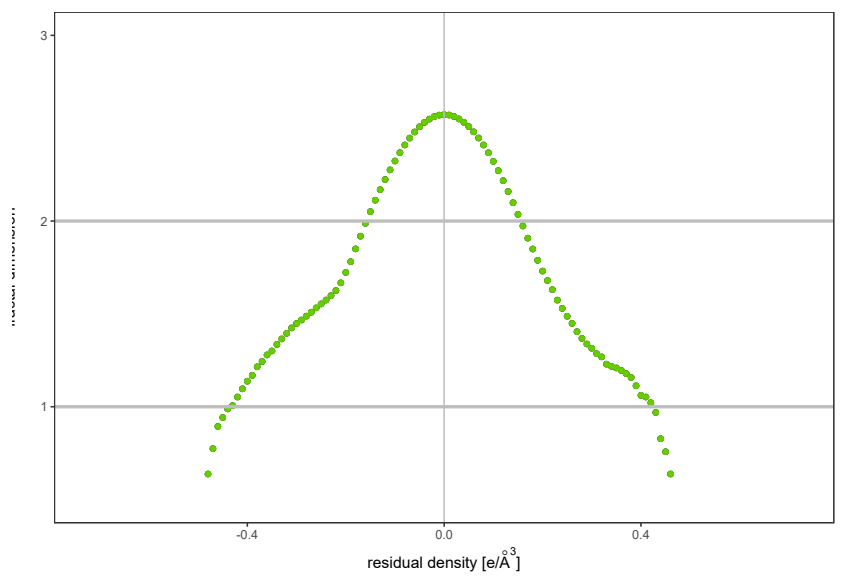

PT2, MM, anharmonic $n=3,4, H$ positions unrestrained, $|F| \geq 2 \sigma(|F|)$

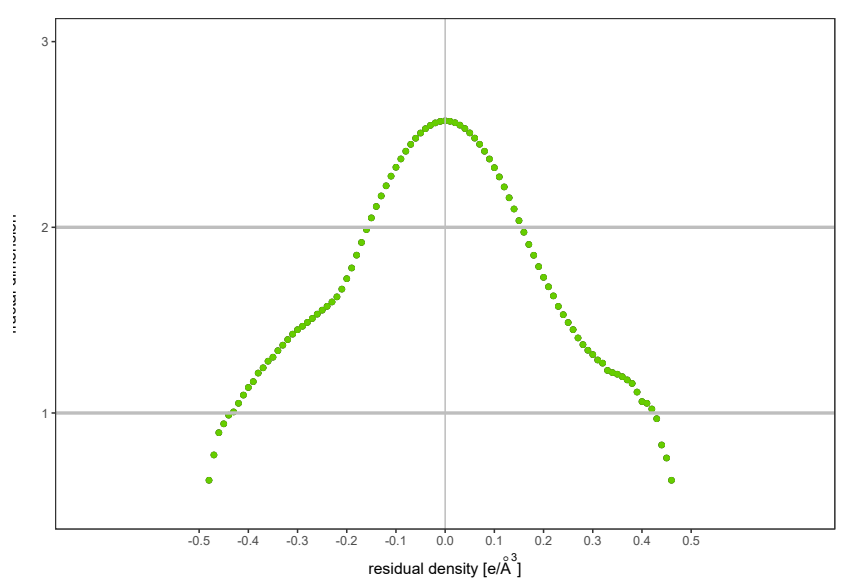

PT2, MM, harmonic, H positions unrestrained, no intensity cutoff

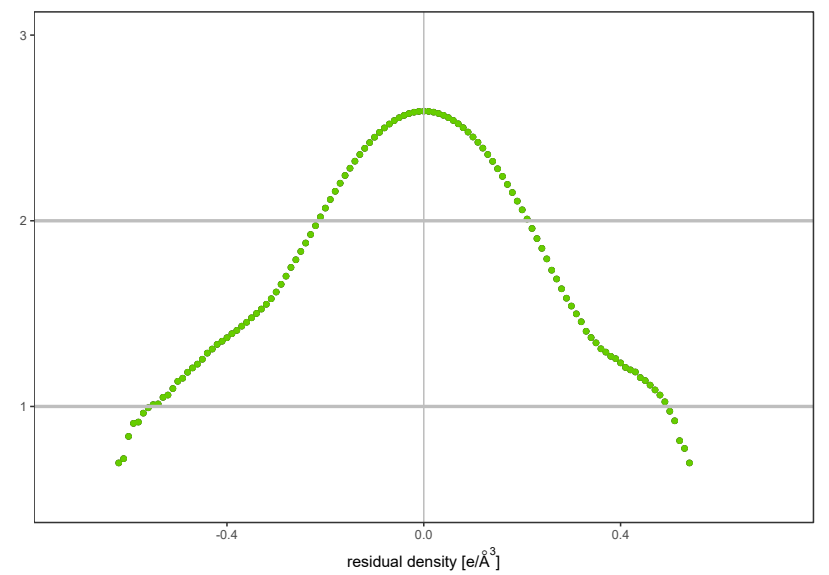

PT2, MM, anharmonic $n=3, H$ positions unrestrained, no intensity cutoff

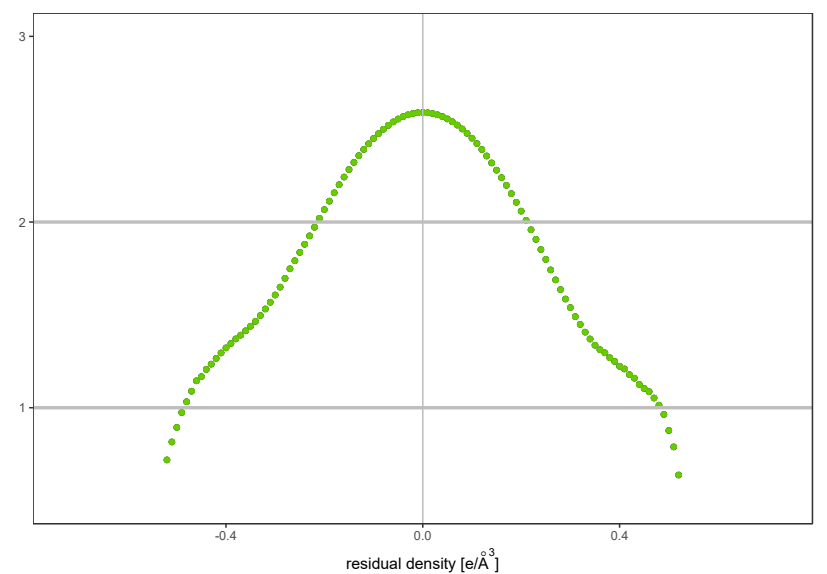

PT2, MM, anharmonic $n=3,4, H$ positions unrestrained, no intensity cutoff

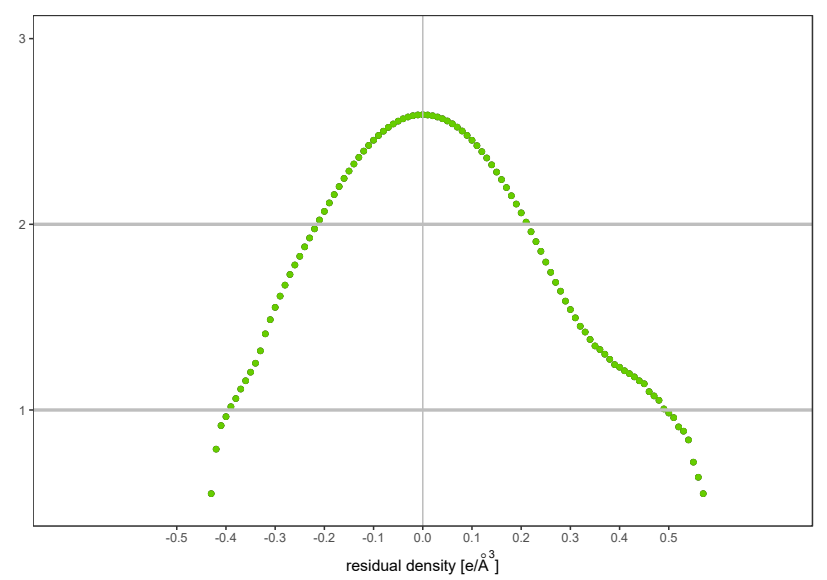

Figure 8: Fractal dimension plots for PT-2, MM, unrestrained X-H distances, left: $|F| \geq 2 \sigma(|F|)$ and right: all reflections included. 
PT8, HAR, harmonic, $|F| \geq 2 \sigma(|F|)$

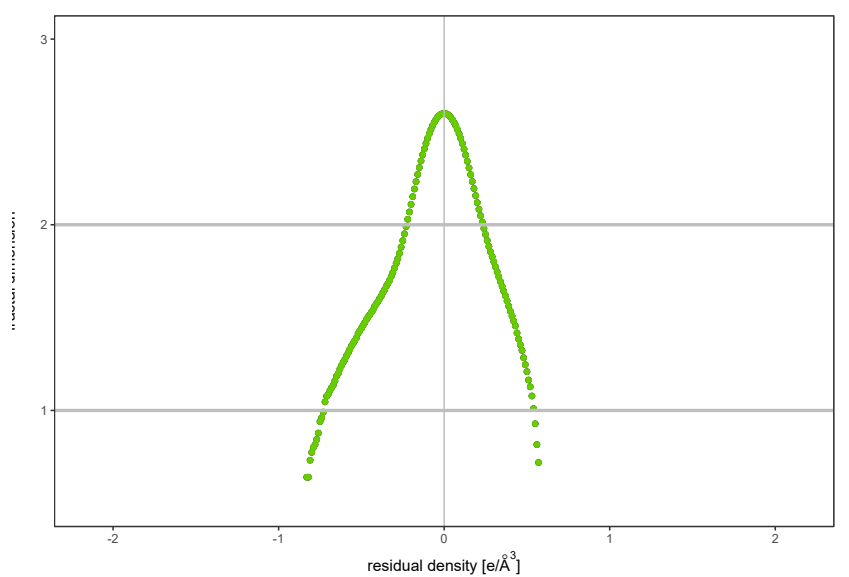

PT8, HAR, anharmonic $n=3,|F| \geq 2 \sigma(|F|) n$

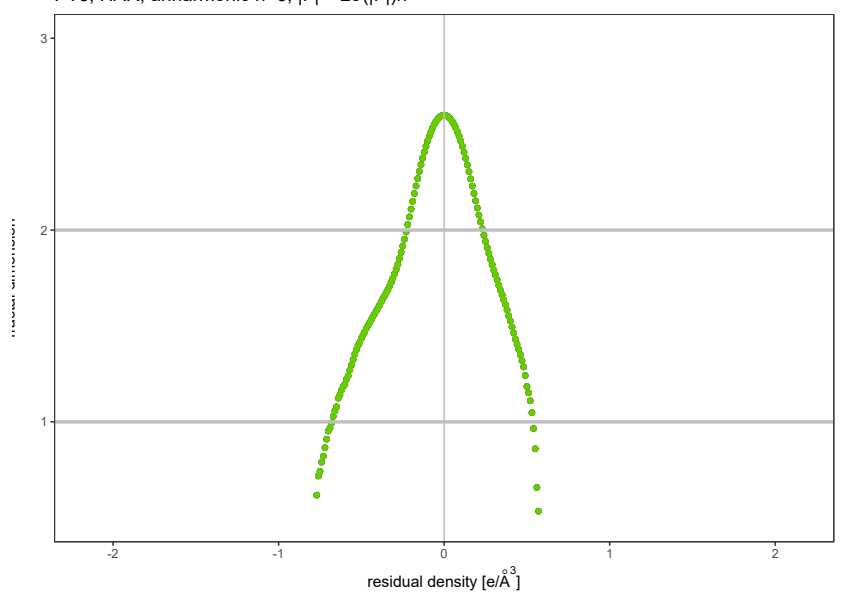

PT8, HAR, anharmonic $n=3,4,|F| \geq 2 \sigma(|F|)$

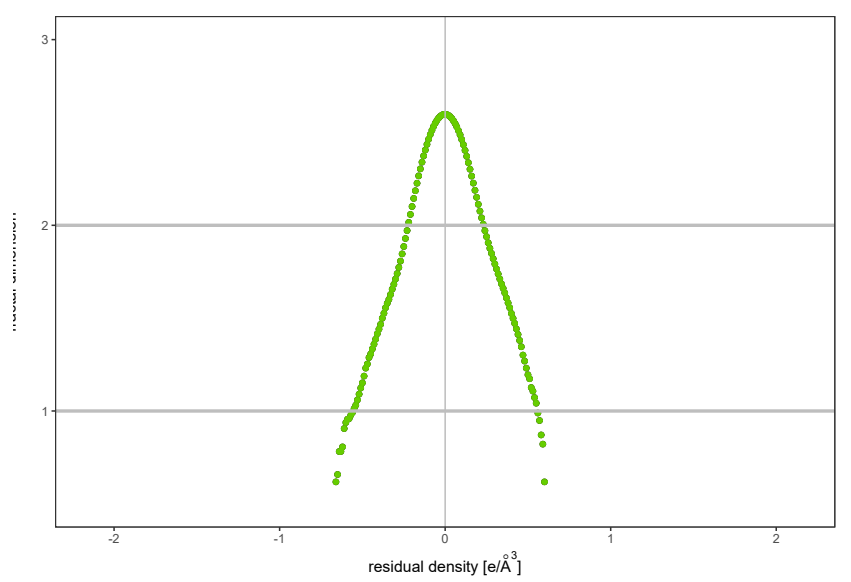

PT8, HAR, harmonic, no intensity cutoff

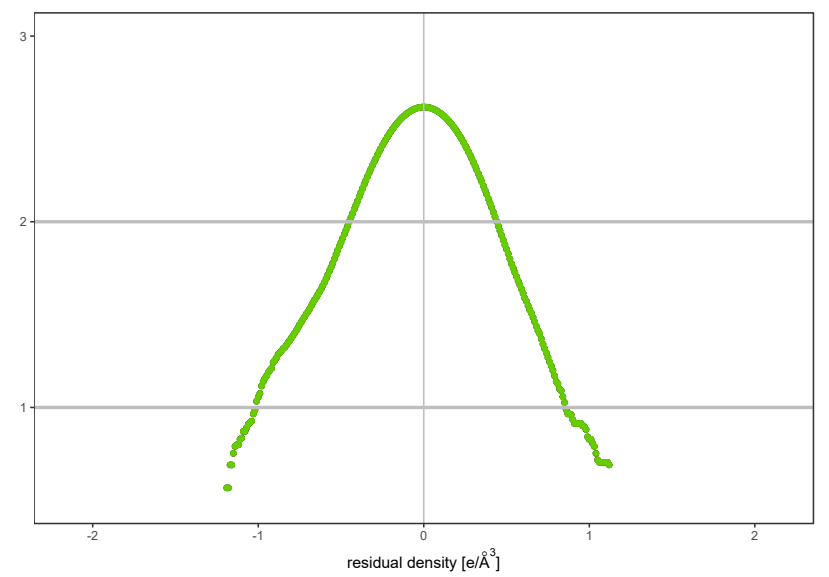

PT8, HAR, anharmonic $n=3$, no intensity cutoff

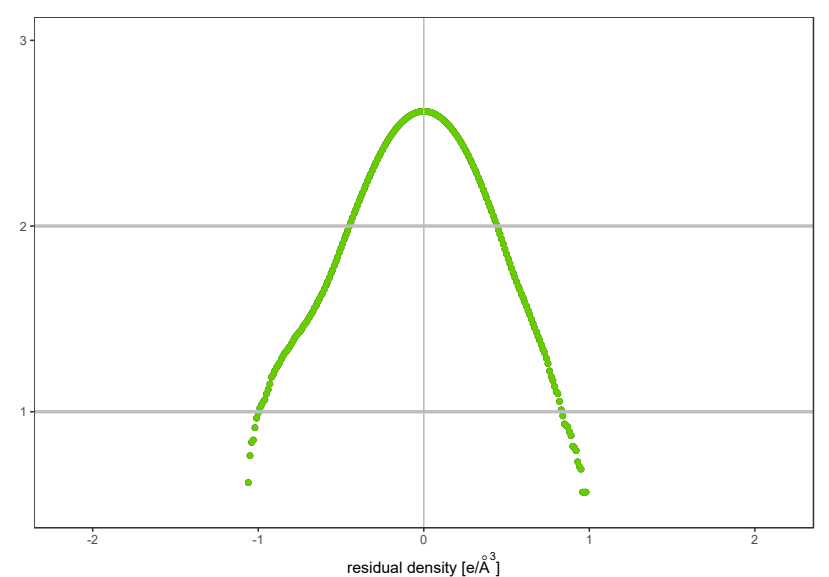

PT8, HAR, anharmonic $n=3,4$, no intensity cutoff

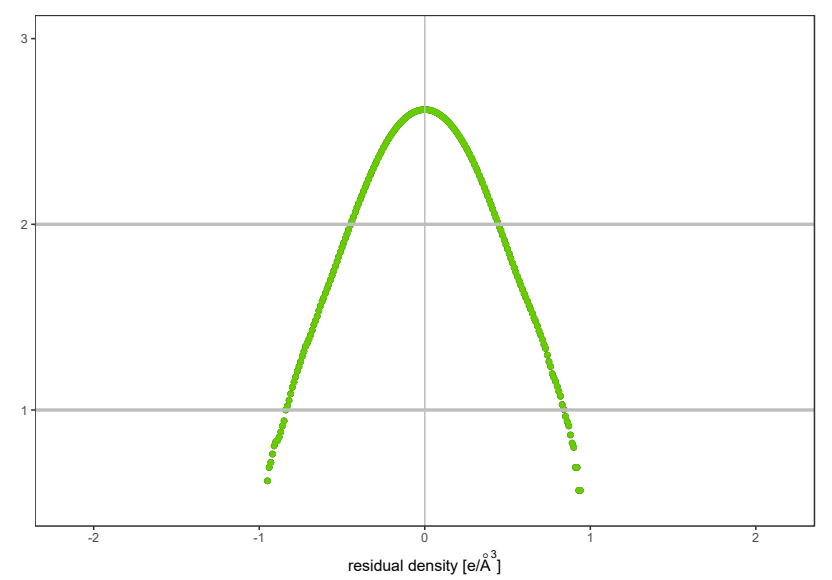

Figure 9: Fractal dimension plots for PT-8, HAR, left: $|F| \geq 2 \sigma(|F|)$ and right: all reflections included. 
PT8, XWR, harmonic, $|\mathrm{F}| \geq 2 \sigma(|\mathrm{F}|)$

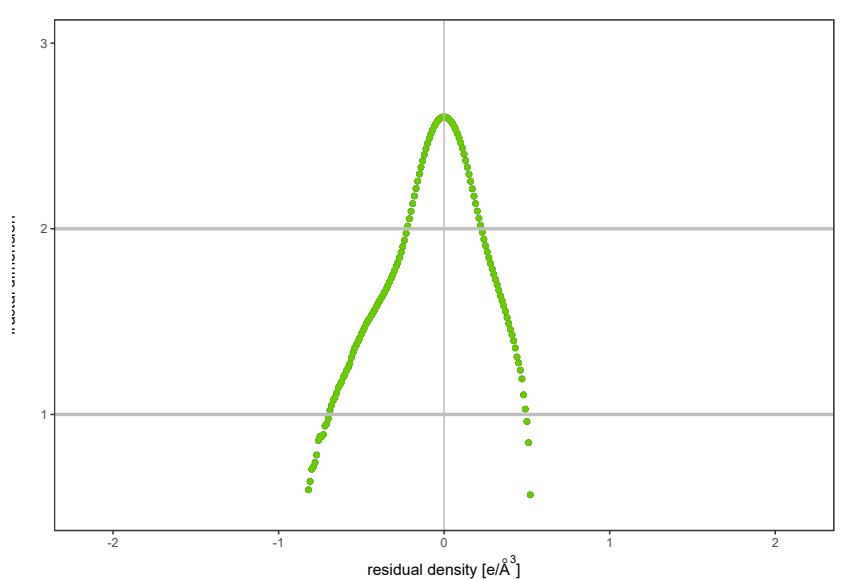

PT8, XWR, anharmonic $n=3,4,|F| \geq 2 \sigma(|F|)$

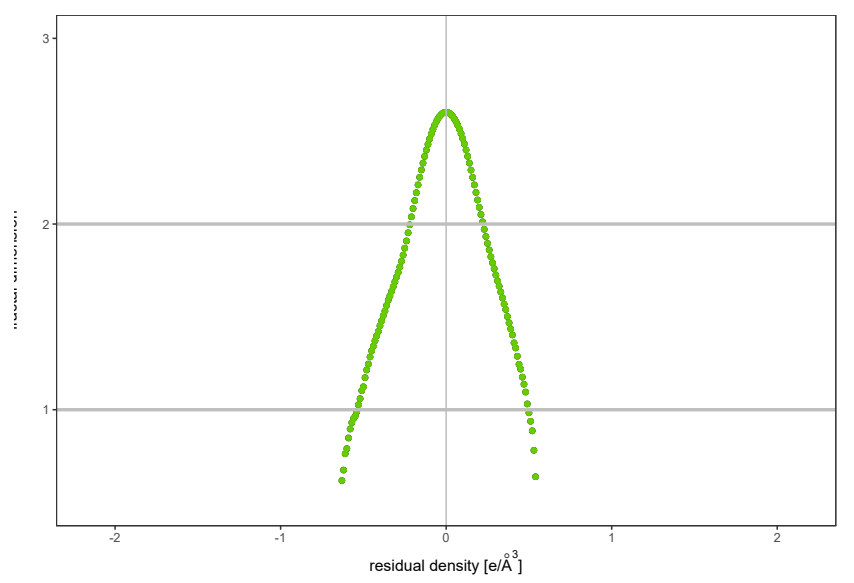

PT8, XWR, harmonic, no intensity cutoff

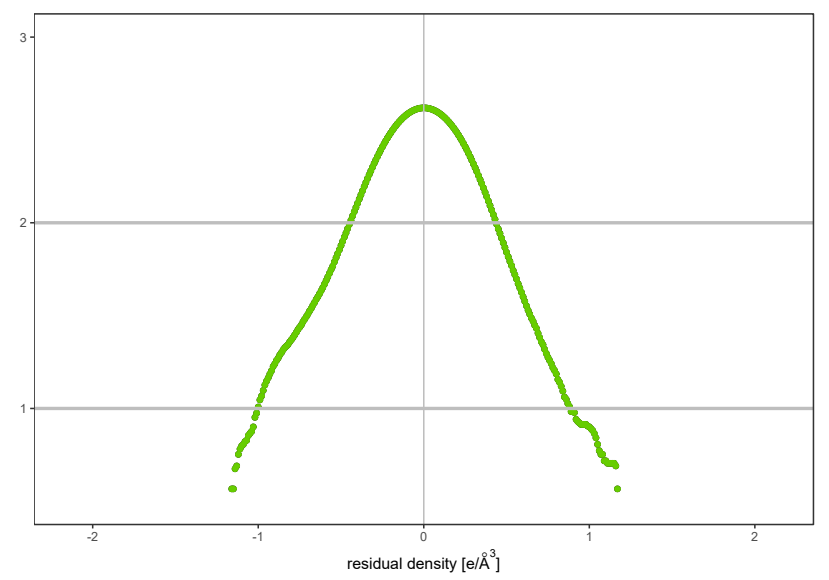

PT8, XWR, anharmonic $n=3,4$, no intensity cutoff

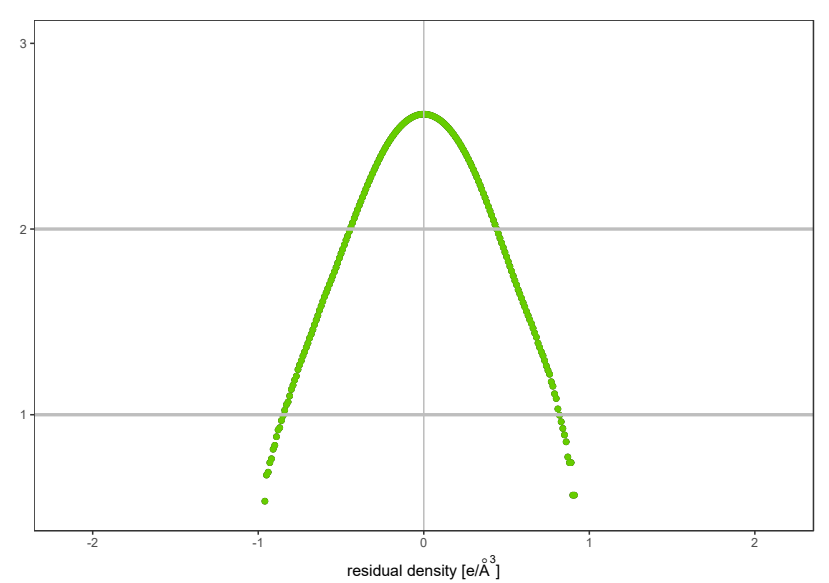

Figure 10: Fractal dimension plots for PT-8, XWR, left: $|F| \geq 2 \sigma(|F|)$ and right: all reflections included. 
PT8, MM, harmonic, $|F| \geq 2 \sigma(|F|)$

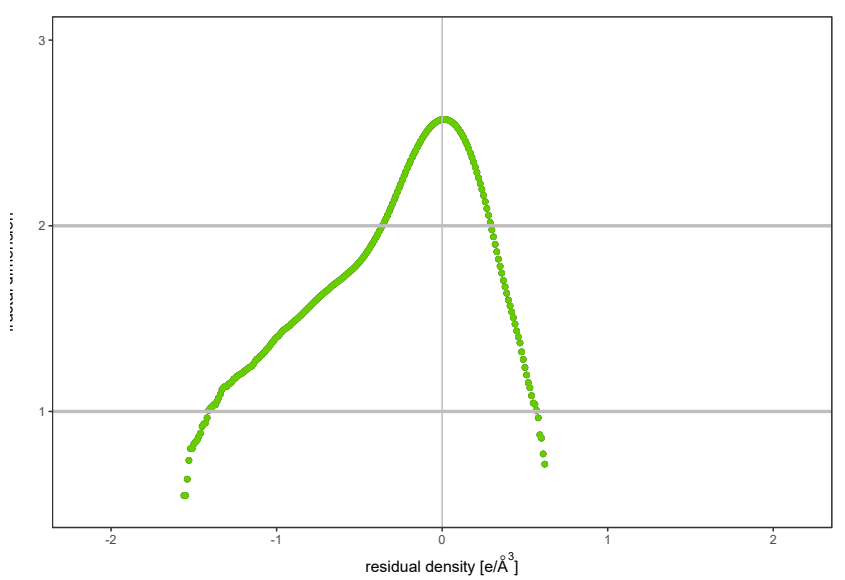

PT8, MM, anharmonic $n=3,|F| \geq 2 \sigma(|F|)$

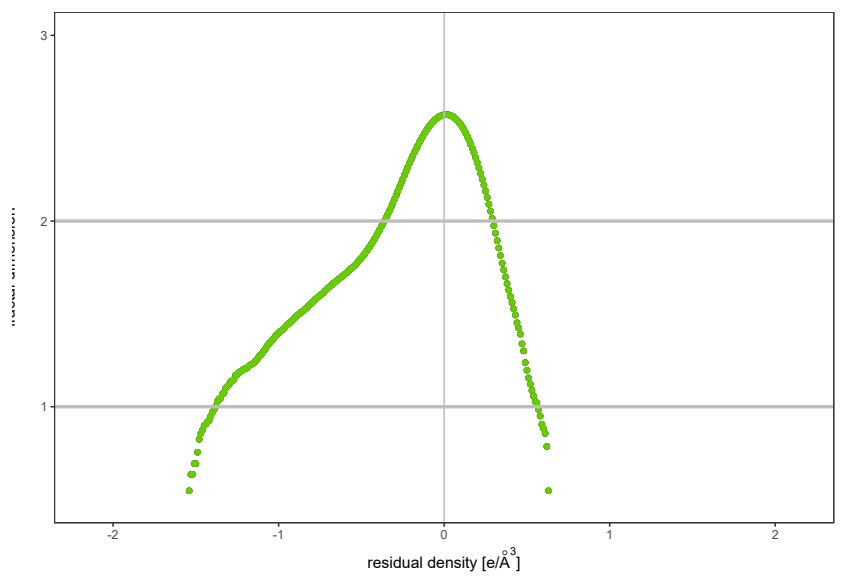

PT8, MM, anharmonic $n=3,4,|F| \geq 2 \sigma(|F|)$

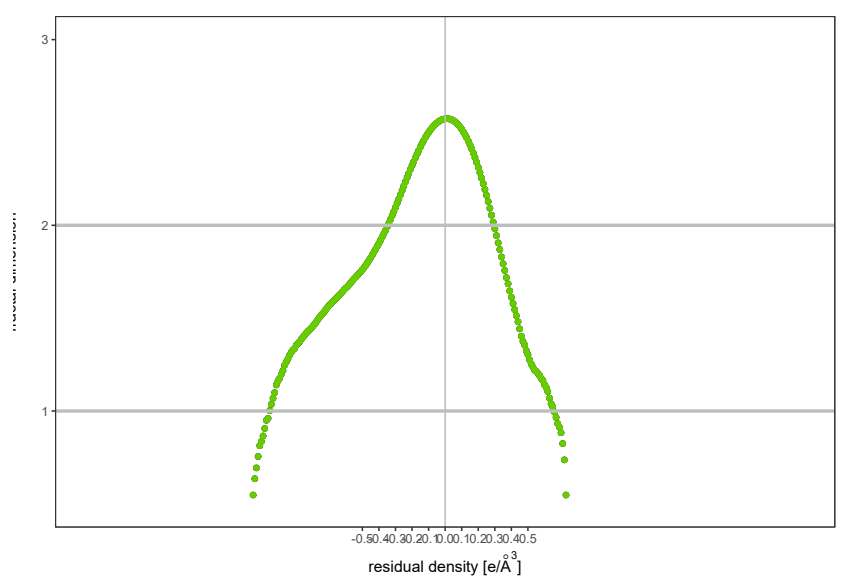

PT8, MM, harmonic, no intensity cutoff

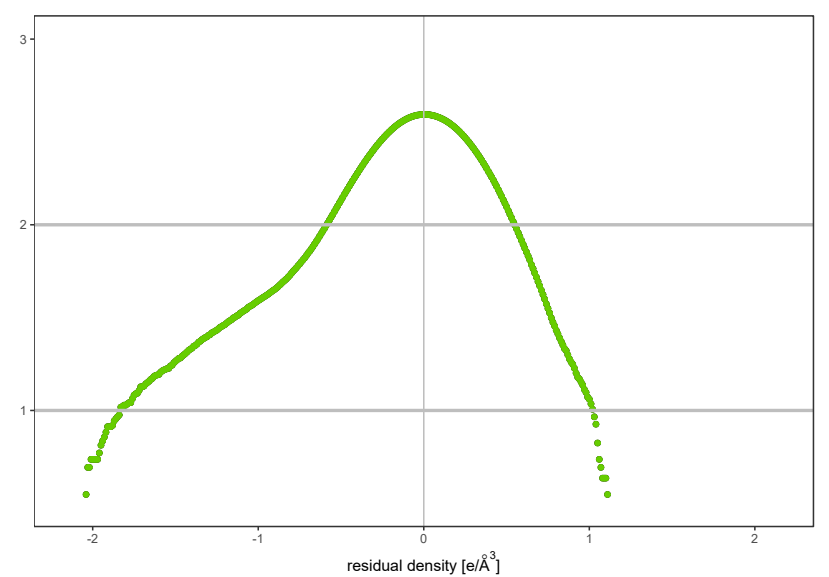

PT8, MM, anharmonic $n=3$, no intensity cutoff

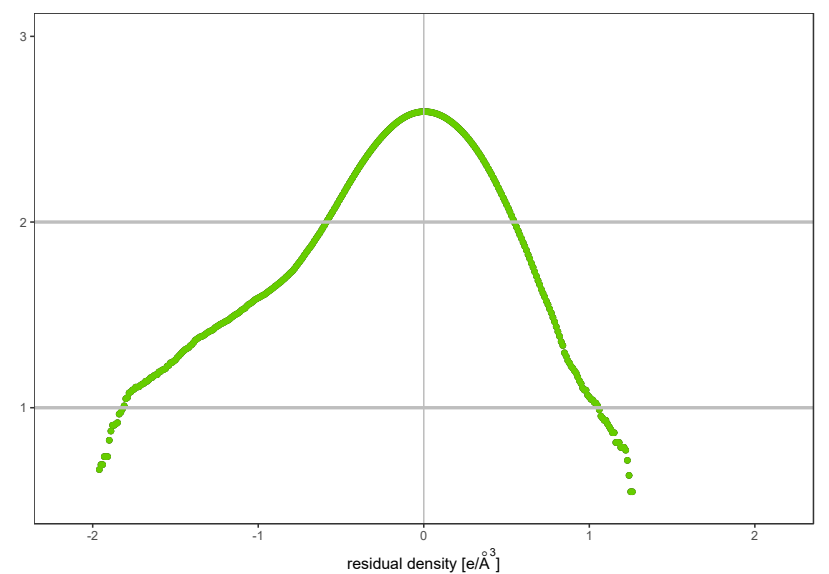

PT8, MM, anharmonic $n=3,4$, no intensity cutoff

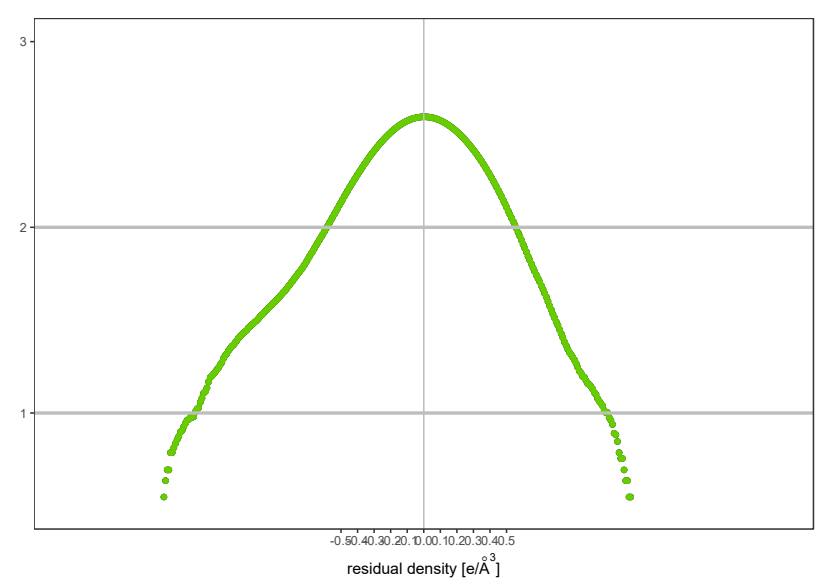

Figure 11: Fractal dimension plots for PT-8, MM, restrained X-H distances, left: $|F| \geq 2 \sigma(|F|)$ and right: all reflections included. 
PT10, HAR, harmonic, $|F| \geq 2 \sigma(|F|)$

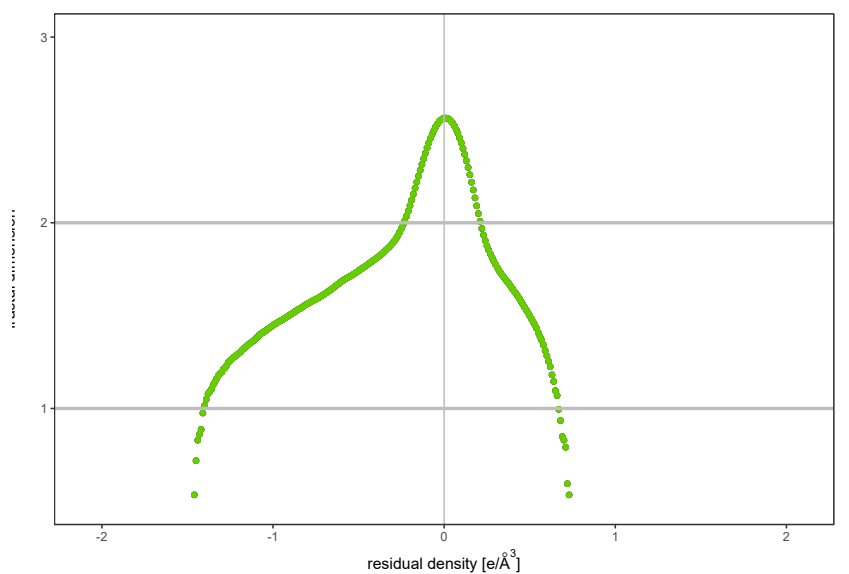

PT10, HAR, anharmonic $n=3,|F| \geq 2 \sigma(|F|) n$

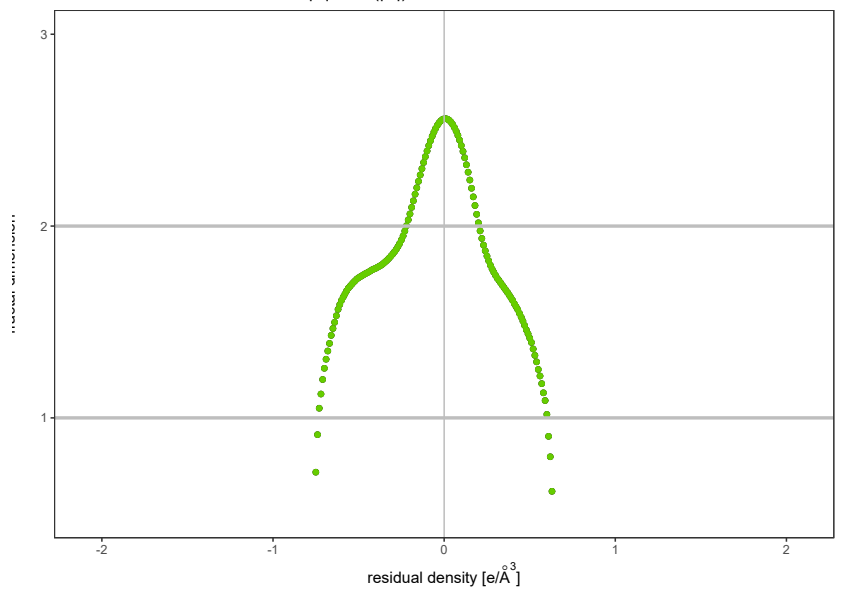

PT10, HAR, anharmonic $n=3,4,|F| \geq 2 \sigma(|F|)$

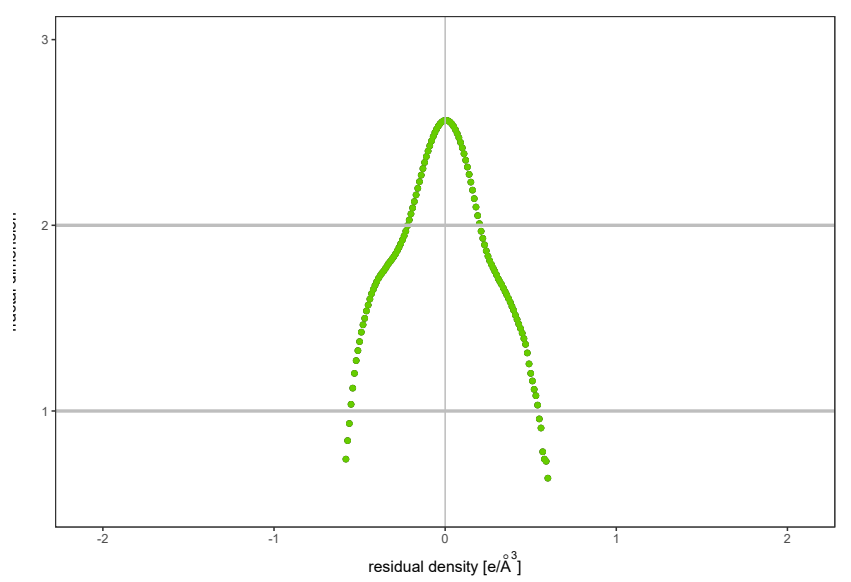

PT10, HAR, harmonic, no intensity cutoff

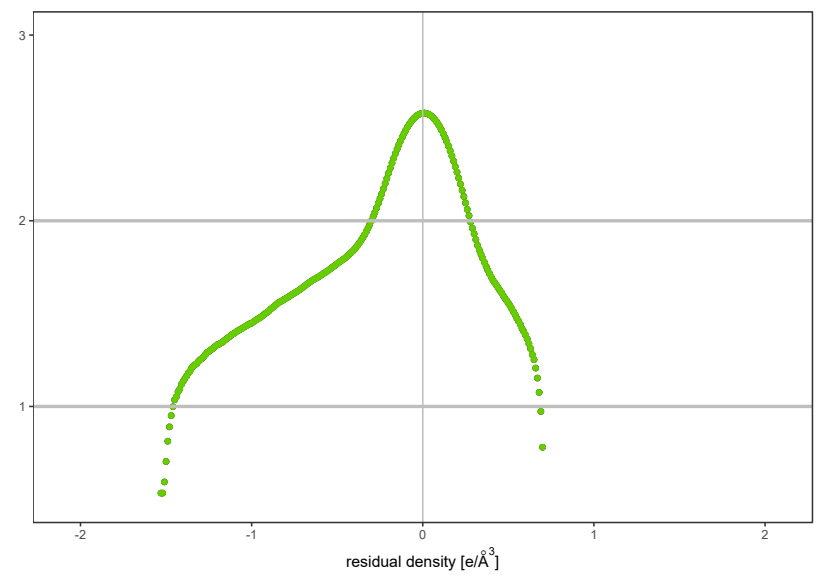

PT10, HAR, anharmonic $n=3$, no intensity cutoff

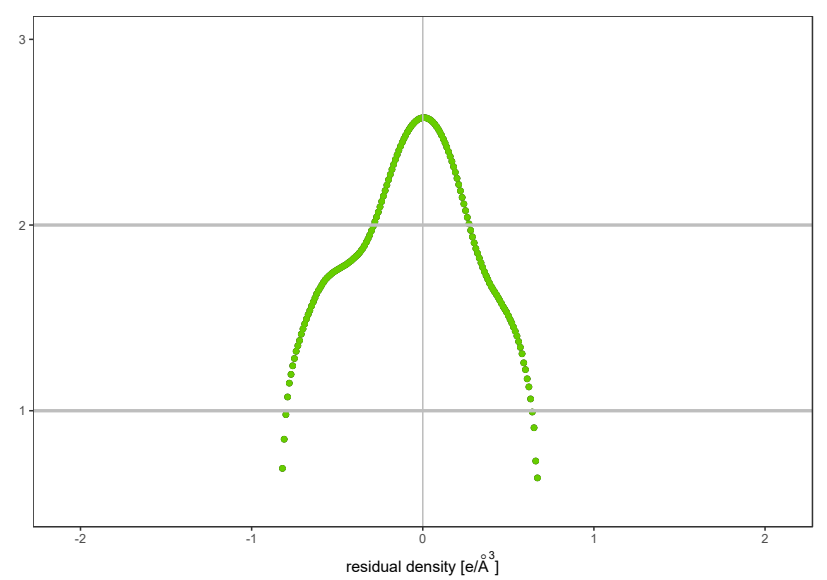

PT10, HAR, anharmonic $n=3,4$, no intensity cutoff

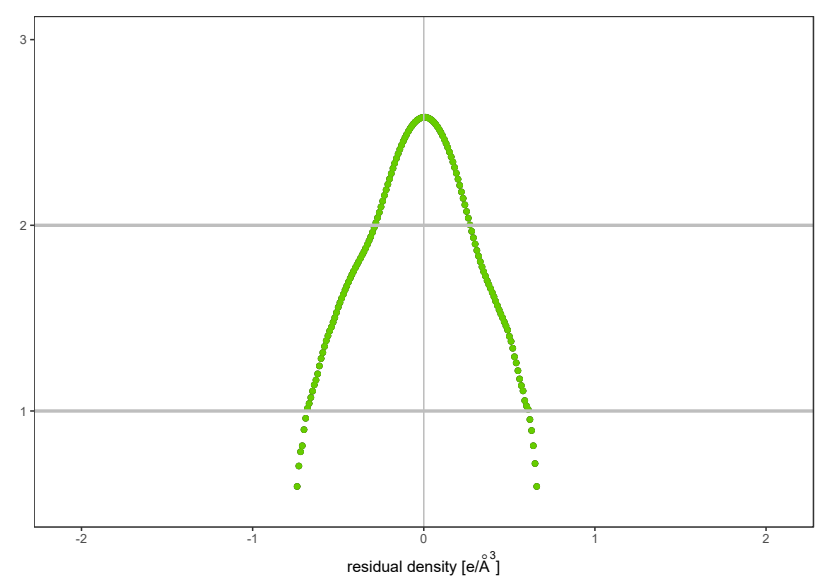

Figure 12: Fractal dimension plots for PT-10, HAR, left: $|F| \geq 2 \sigma(|F|)$ and right: all reflections included. 
PT10, XWR, harmonic, $|F| \geq 2 \sigma(|F|)$

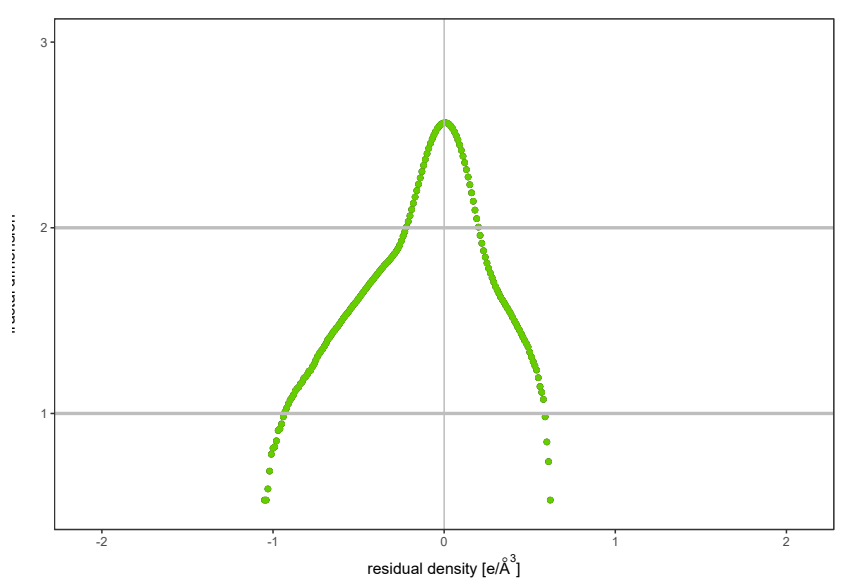

PT10, XWR, anharmonic $n=3,4,|F| \geq 2 \sigma(|F|)$

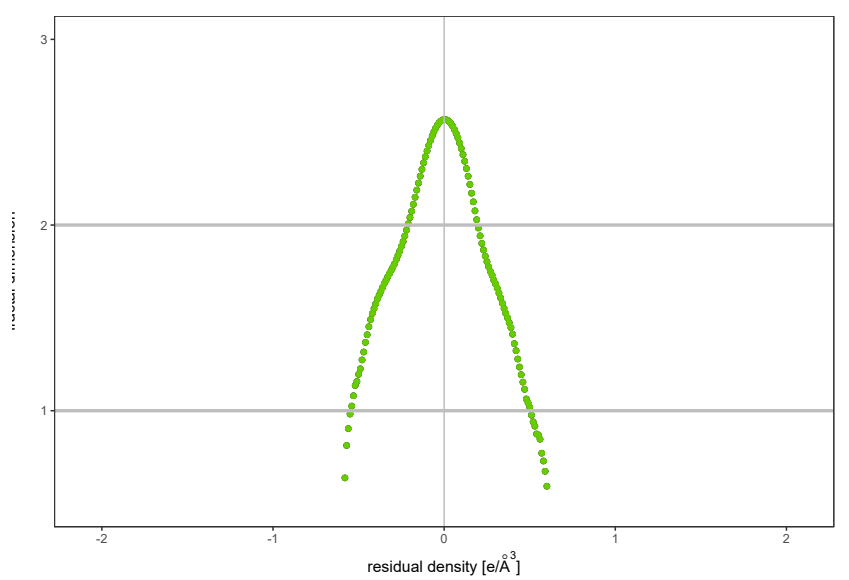

PT10, XWR, harmonic, no intensity cutoff

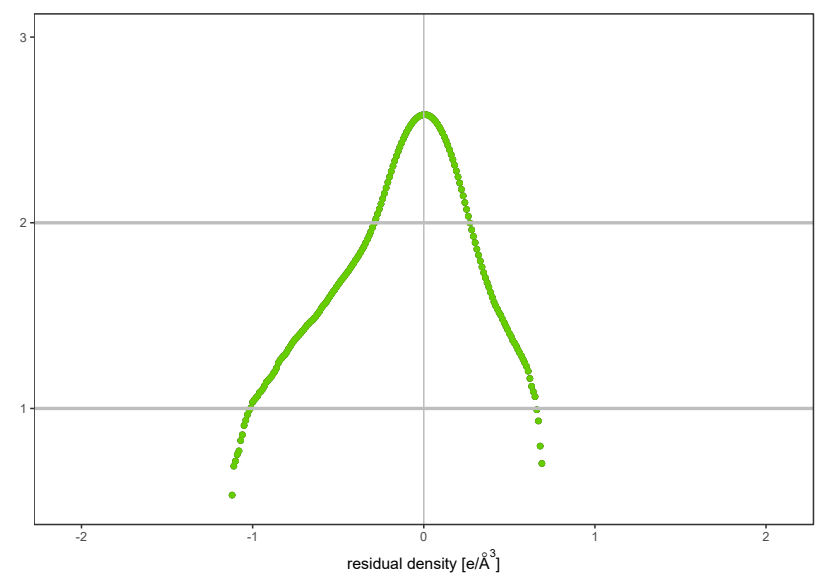

PT10, $X W R$, anharmonic $n=3,4$, no intensity cutoff

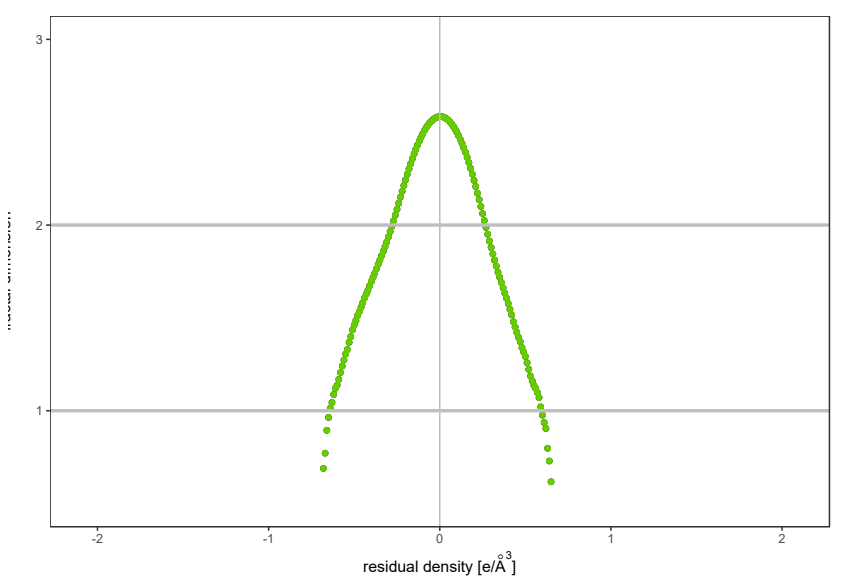

Figure 13: Fractal dimension plots for PT-10, XWR, left: $|F| \geq 2 \sigma(|F|)$ and right: all reflections included. 
PT10, MM, harmonic, $|\mathrm{F}| \geq 2 \sigma(|\mathrm{F}|)$

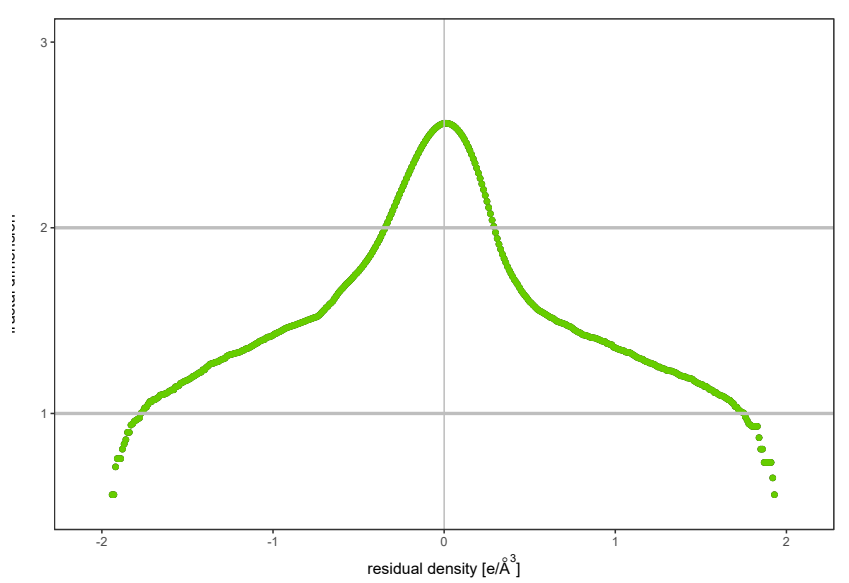

PT10, MM, anharmonic $n=3,|F| \geq 2 \sigma(|F|)$

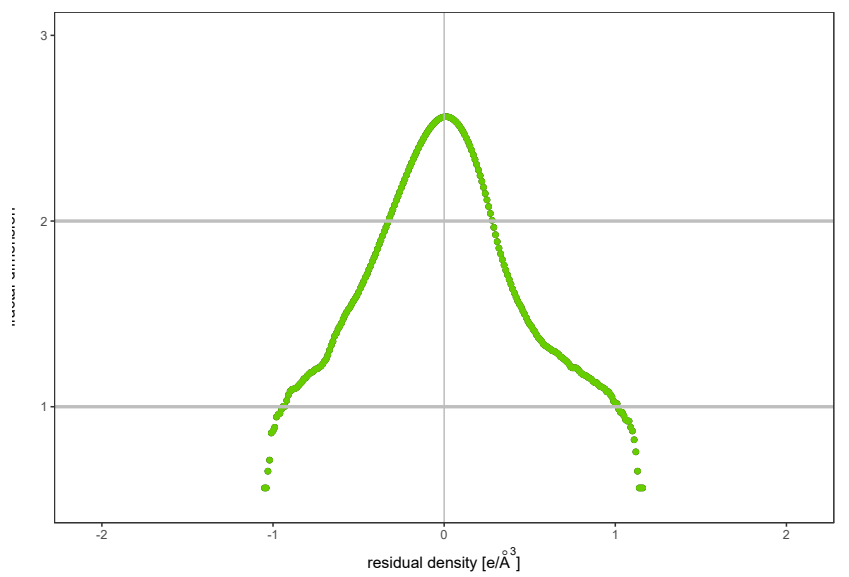

PT10, MM, anharmonic $n=3,4,|F| \geq 2 \sigma(|F|)$

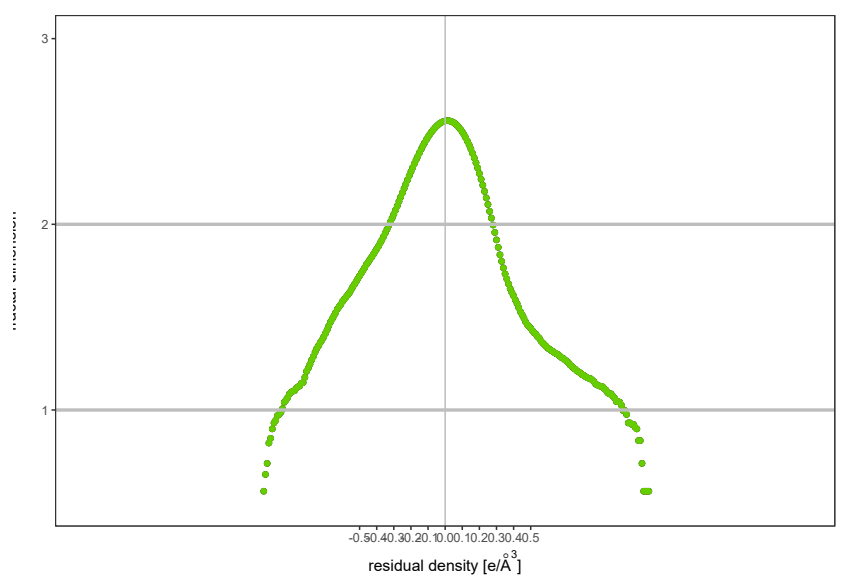

PT10, MM, harmonic, no intensity cutoff

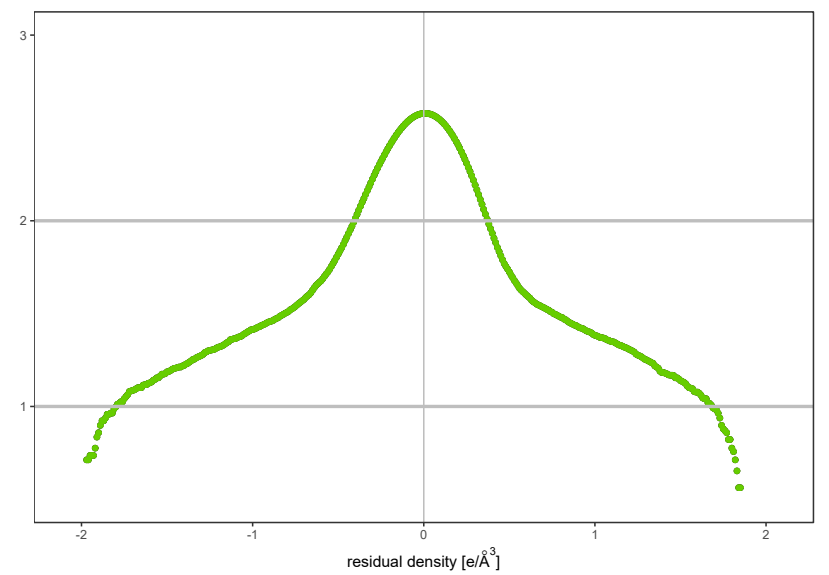

PT10, MM, anharmonic n=3, no intensity cutoff

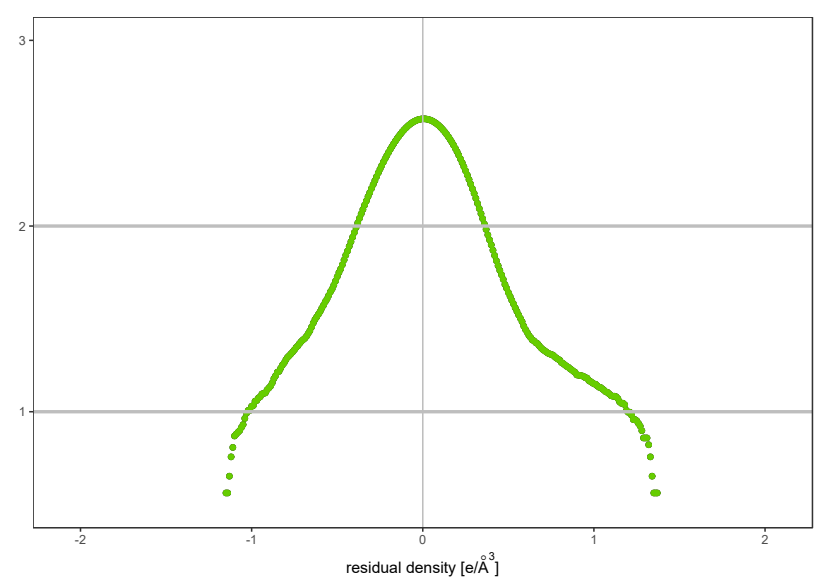

PT10, MM, anharmonic n=3,4, no intensity cutoff

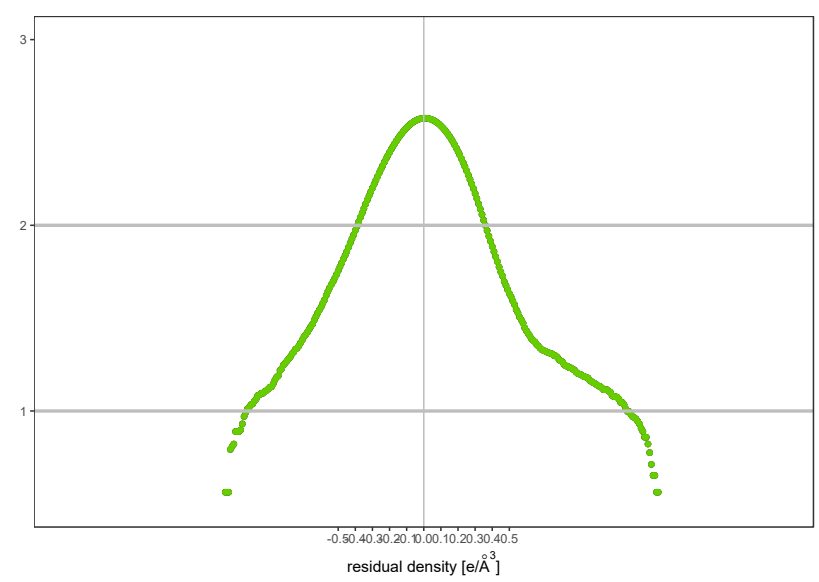

Figure 14: Fractal dimension plots for PT-10, MM, restrained X-H distances, left: $|F| \geq 2 \sigma(|F|)$ and right: all reflections included. 
4 Residaual density maps 


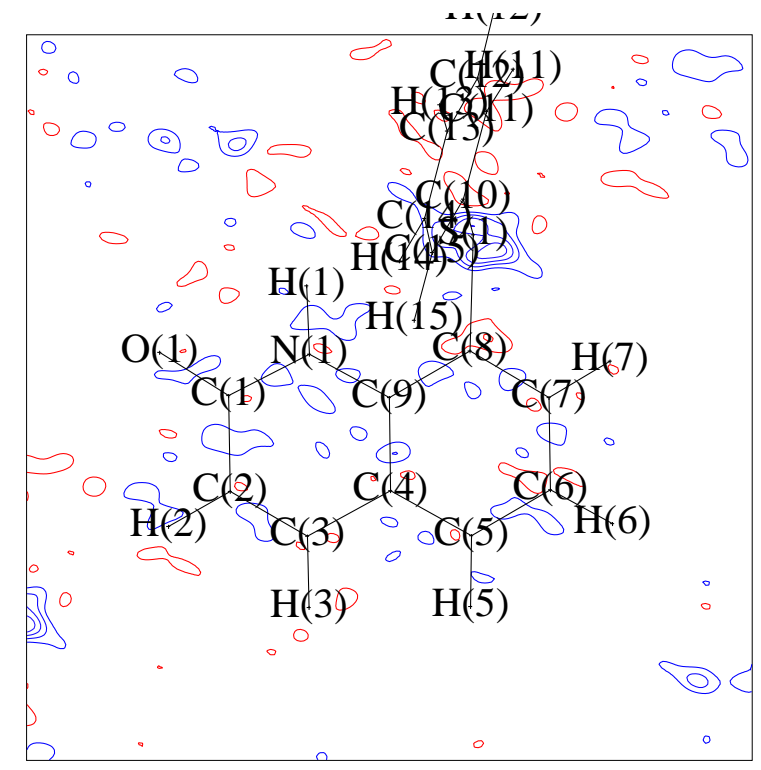

(a) HAR, harmonic, $|F| \geq 2 \sigma(|F|)$

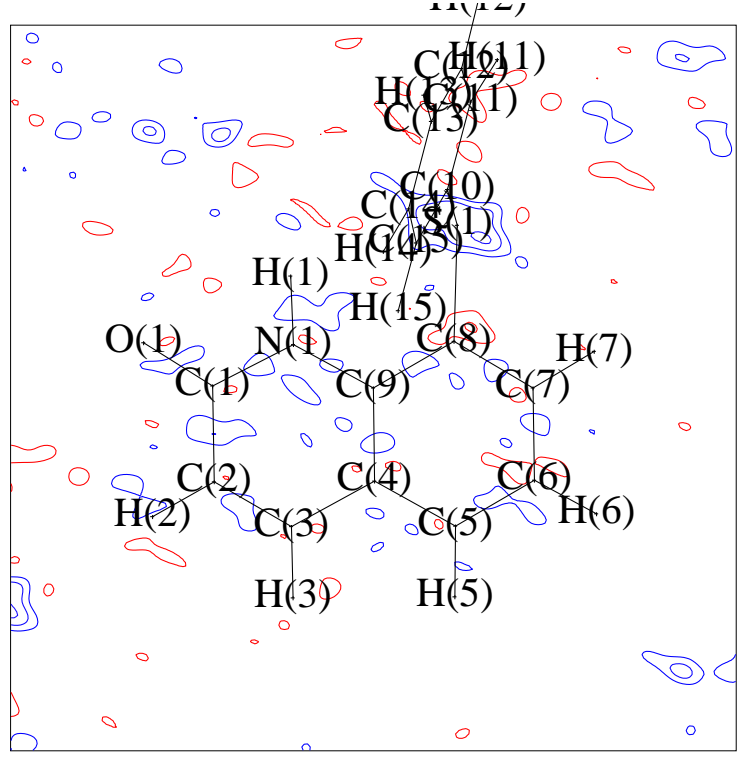

(c) HAR, anharmonic n $=3,|F| \geq 2 \sigma(|F|)$

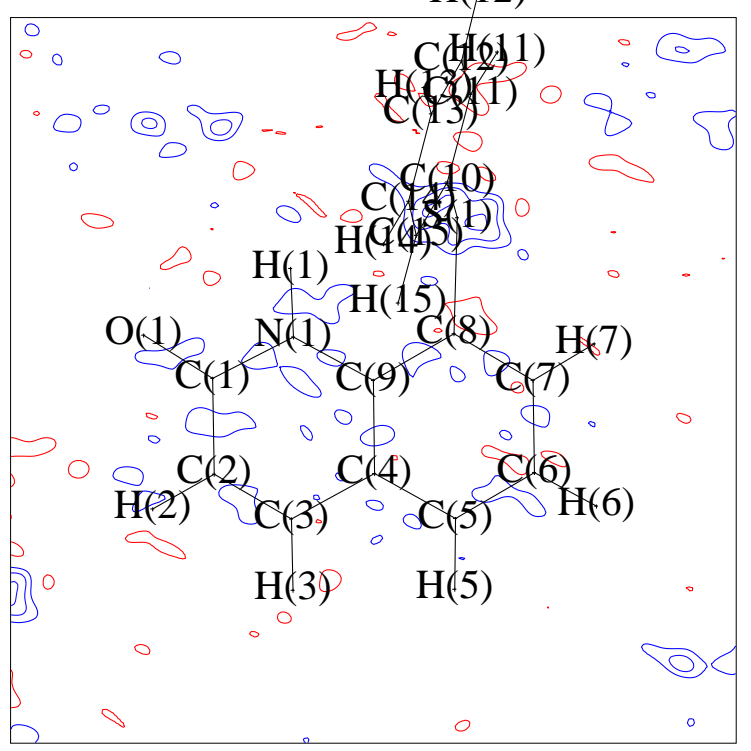

(e) HAR, anharmonic n=4, $|F| \geq 2 \sigma(|F|)$

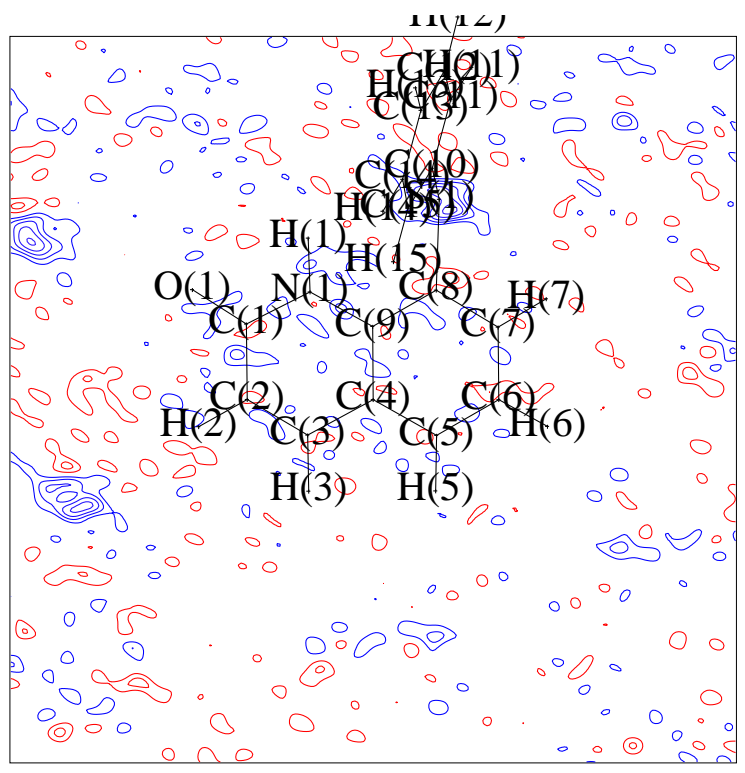

(b) HAR, harmonic, all reflections

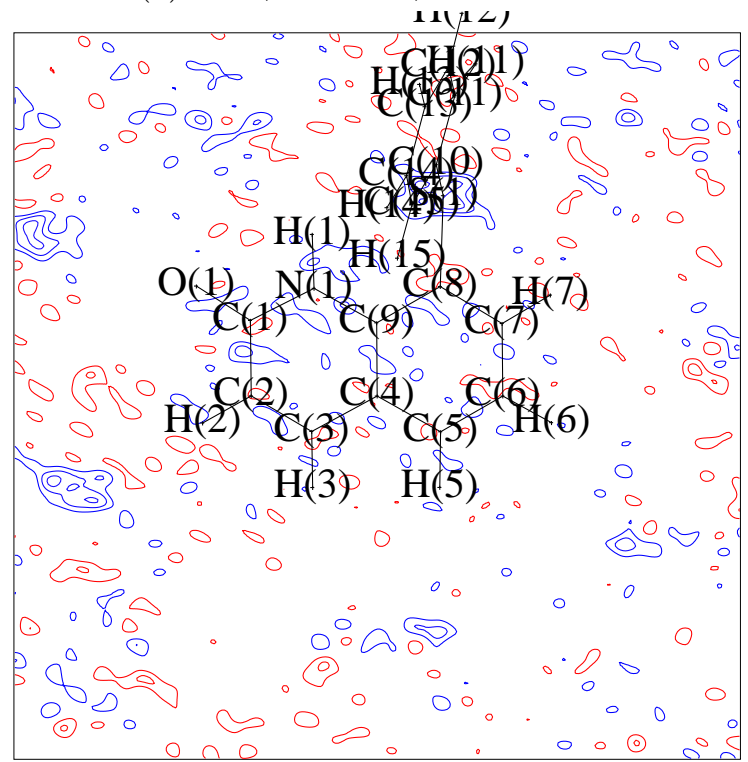

(d) HAR, anharmonic $\mathrm{n}=3$, all reflections

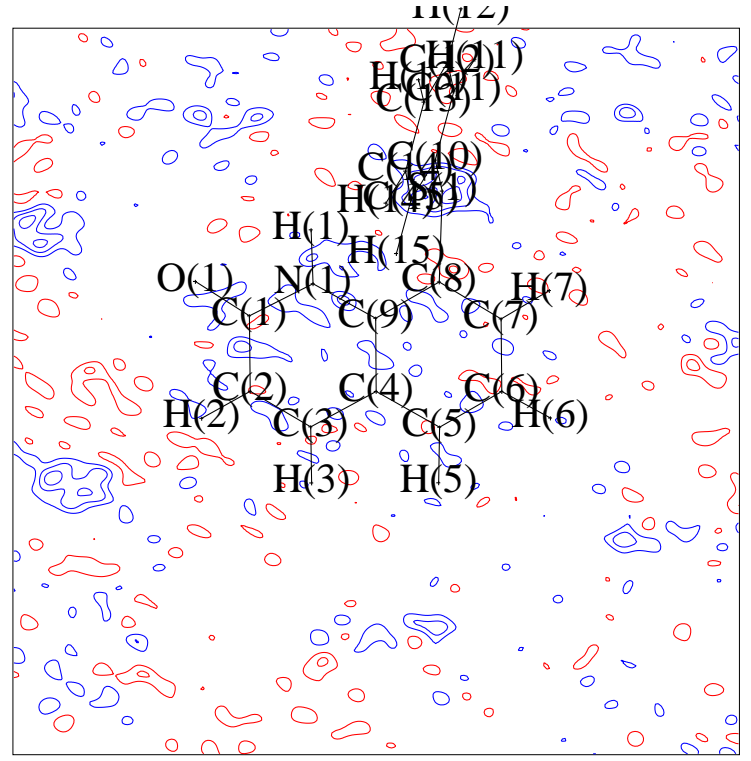

(f) HAR, anharmonic $\mathrm{n}=4$, all reflections

Figure 15: Residual density maps for PT-11 in the plane of quinoline, HAR, left: $|F| \geq 2 \sigma(|F|)$ and right: all reflections included. Contour level: $0.05 \mathrm{e} / \AA^{3}$. Colours: blue - positive, red - negative. 


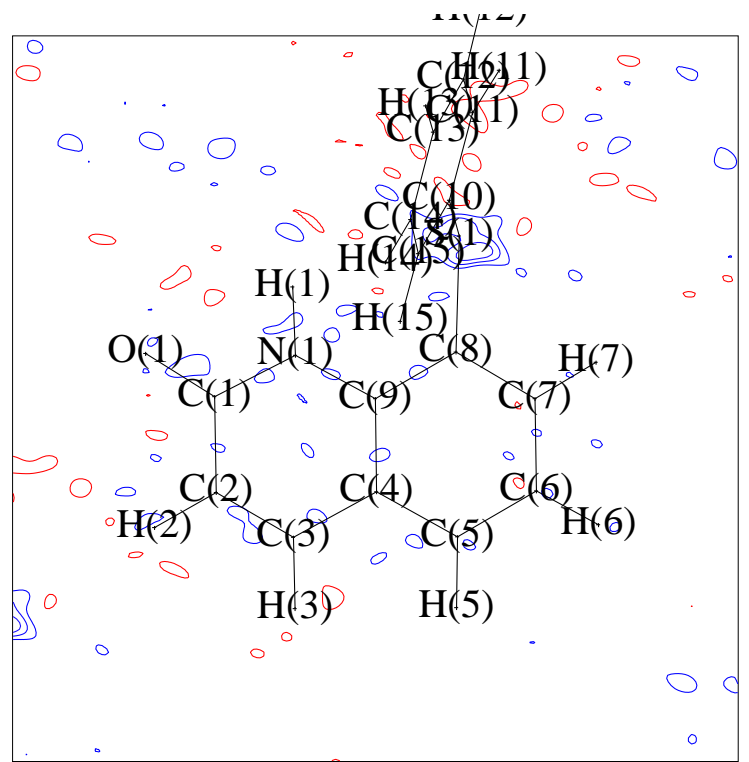

(a) XWR, harmonic, $|F| \geq 2 \sigma(|F|)$

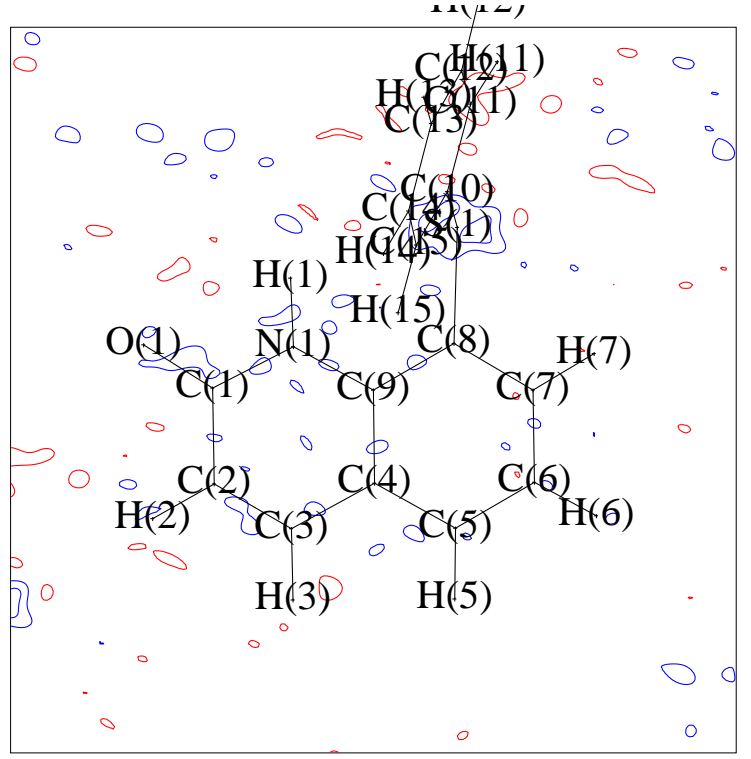

(c) XWR, anharmonic $\mathrm{n}=4,|F| \geq 2 \sigma(|F|)$

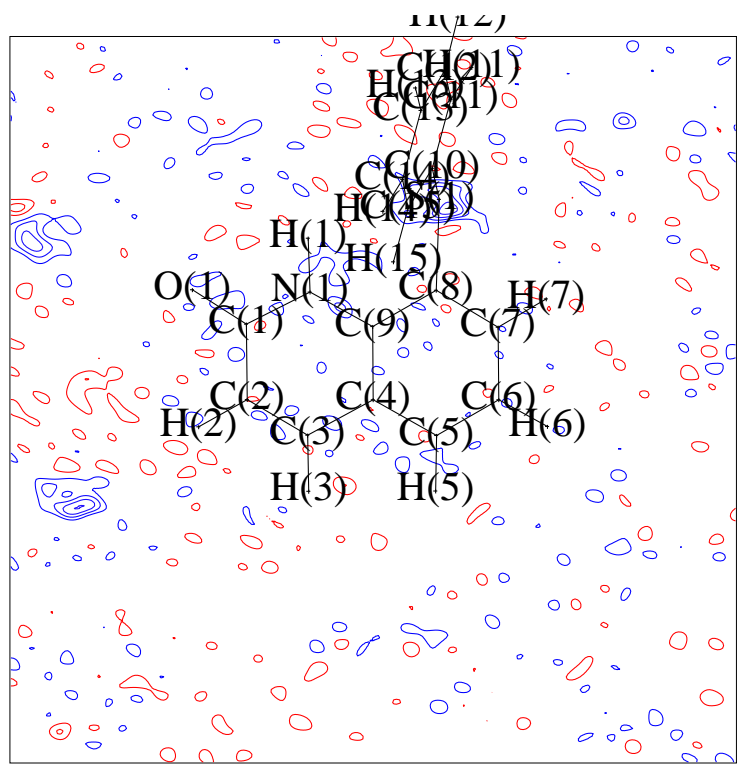

(b) XWR, harmonic, all reflections

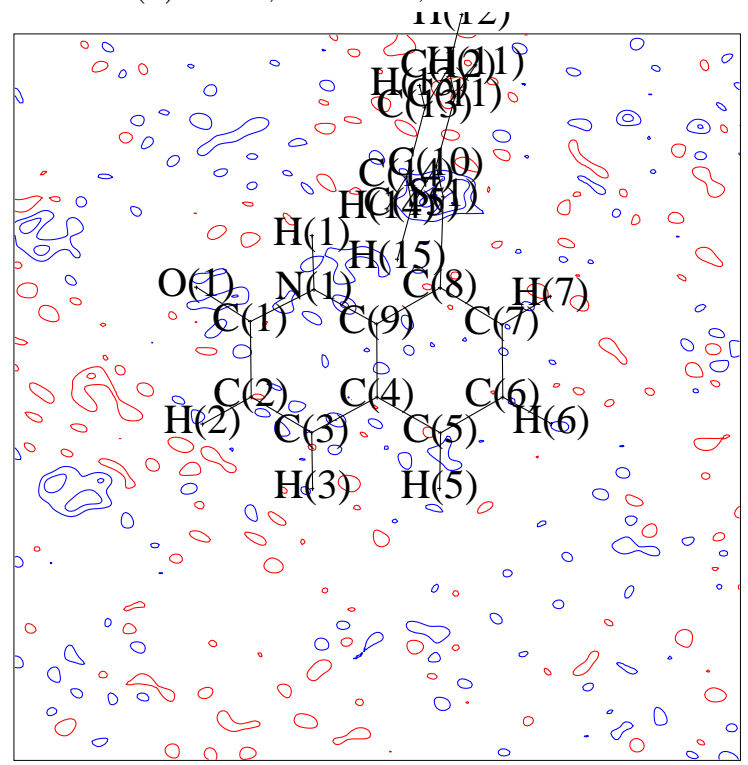

(d) XWR, anharmonic $\mathrm{n}=4$, all reflections

Figure 16: Residual density maps for PT-11 in the plane of quinoline, XWR, left: $|F| \geq 2 \sigma(|F|)$ and right: all reflections included. Contour level: $0.05 \mathrm{e} / \AA^{3}$. Colours: blue - positive, red - negative. 


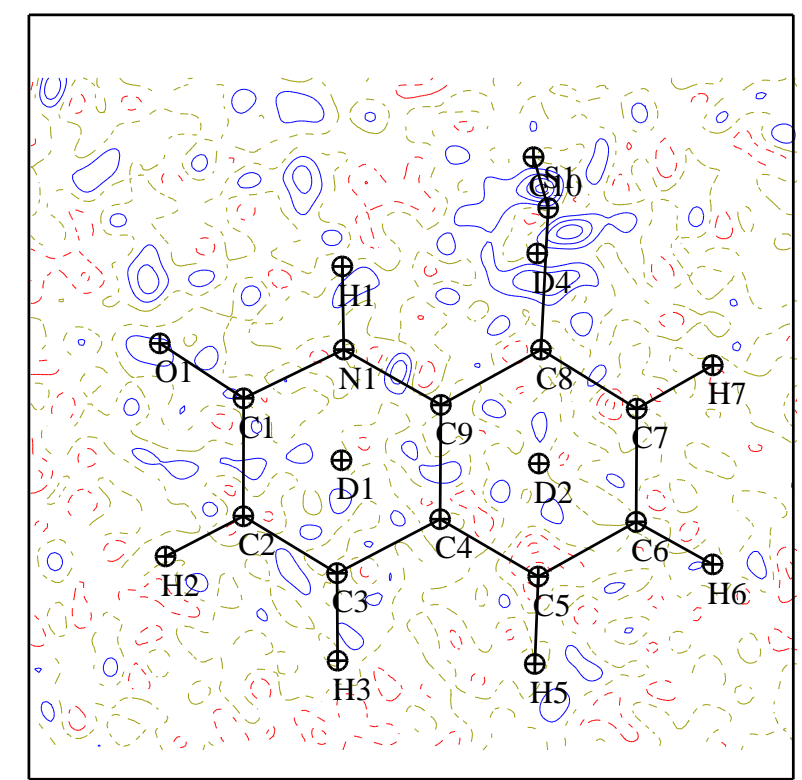

(a) MM, harmonic, $|F| \geq 2 \sigma(|F|)$

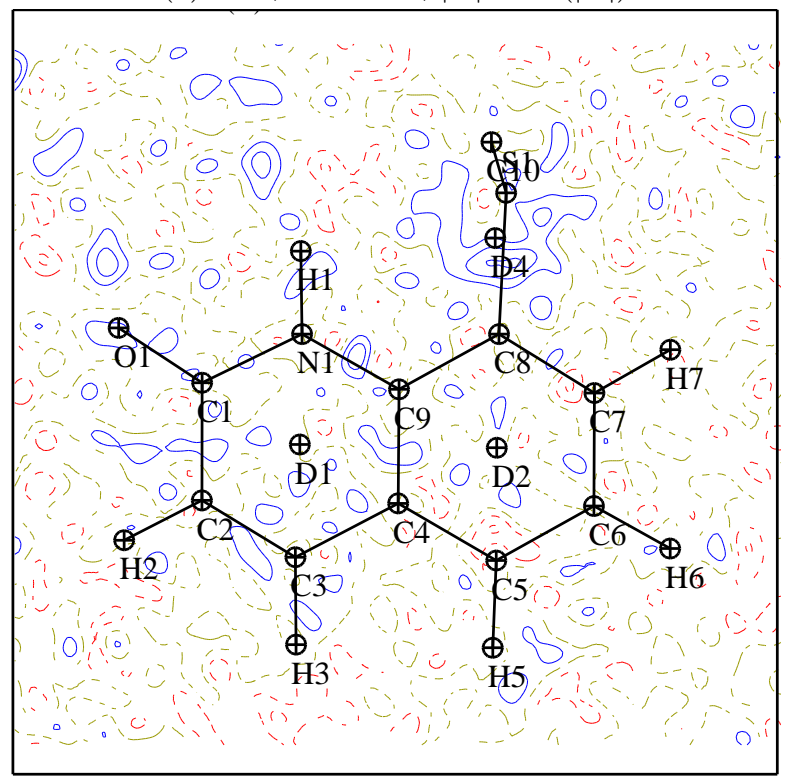

(c) MM, anharmonic $\mathrm{n}=3,|F| \geq 2 \sigma(|F|)$

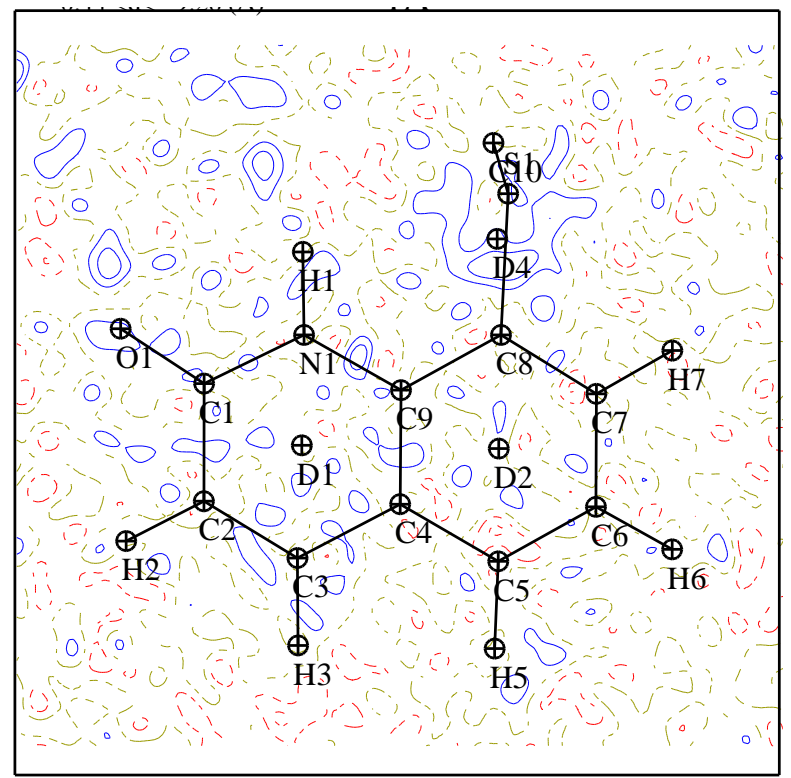

(e) MM, anharmonic $\mathrm{n}=4,|F| \geq 2 \sigma(|F|)$

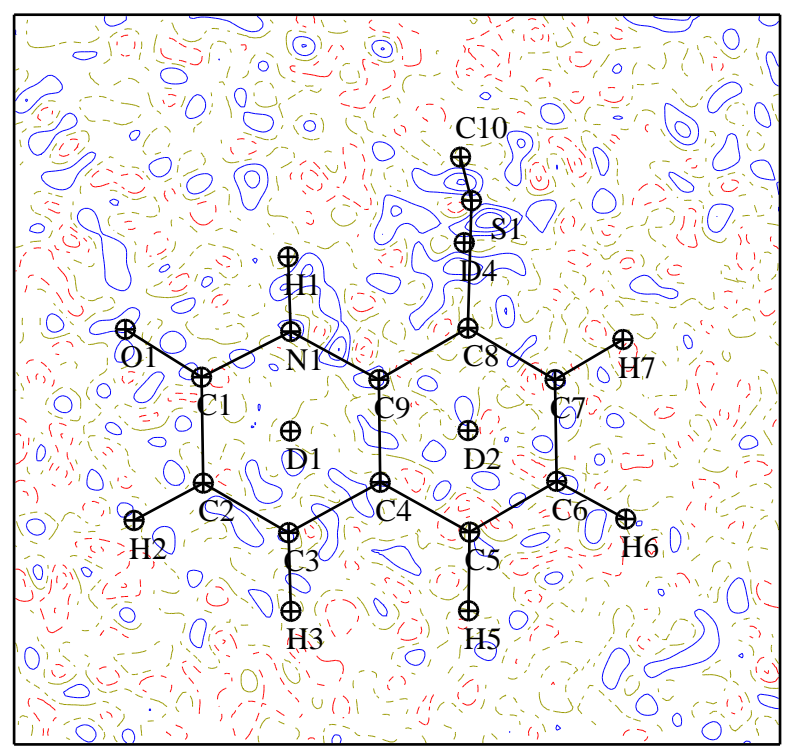

(b) MM, harmonic, all reflections

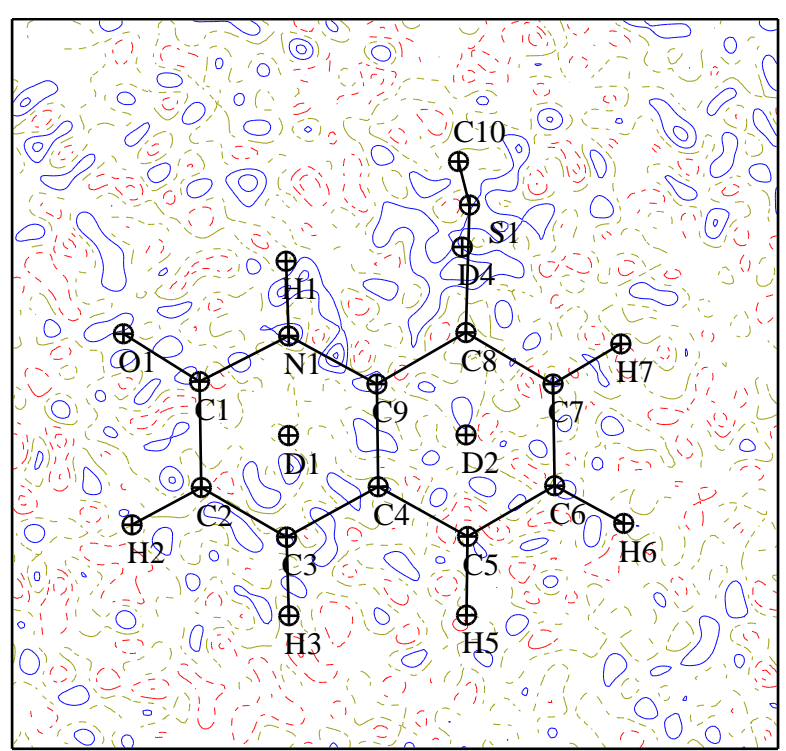

(d) $\mathrm{MM}$, anharmonic $\mathrm{n}=3$, all reflections

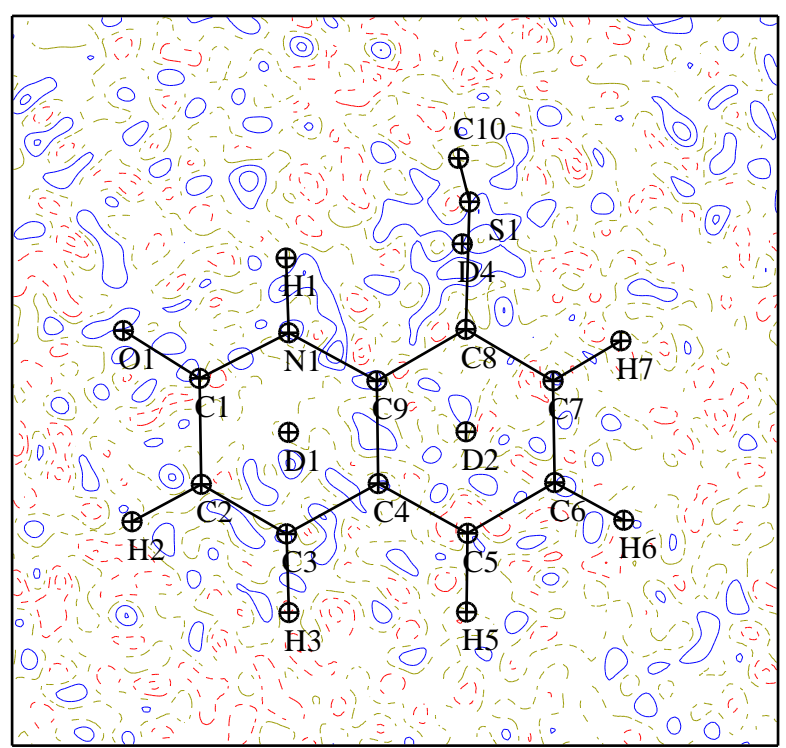

(f) $\mathrm{MM}$, anharmonic $\mathrm{n}=4$, all reflections

Figure 17: Residual density maps for PT-11 in the plane of quinoline, MM, restrained X-H distances, left: $|F| \geq 2 \sigma(|F|)$ and right: all reflections included. Contour level: 0.05 e/ $\AA^{3}$. Colours: blue - positive, red - negative. 


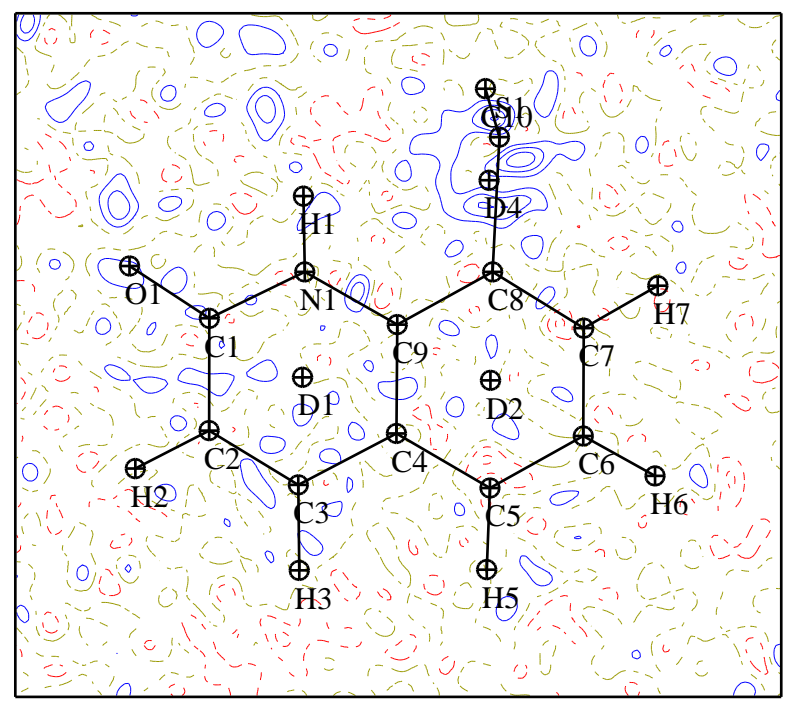

(a) MM, harmonic, $|F| \geq 2 \sigma(|F|)$

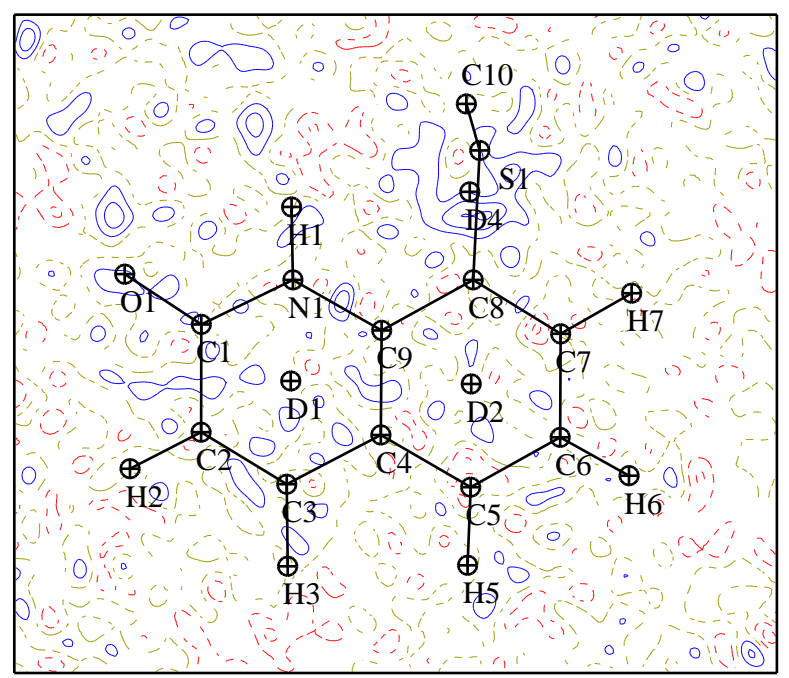

(c) MM, anharmonic $\mathrm{n}=3,|F| \geq 2 \sigma(|F|)$

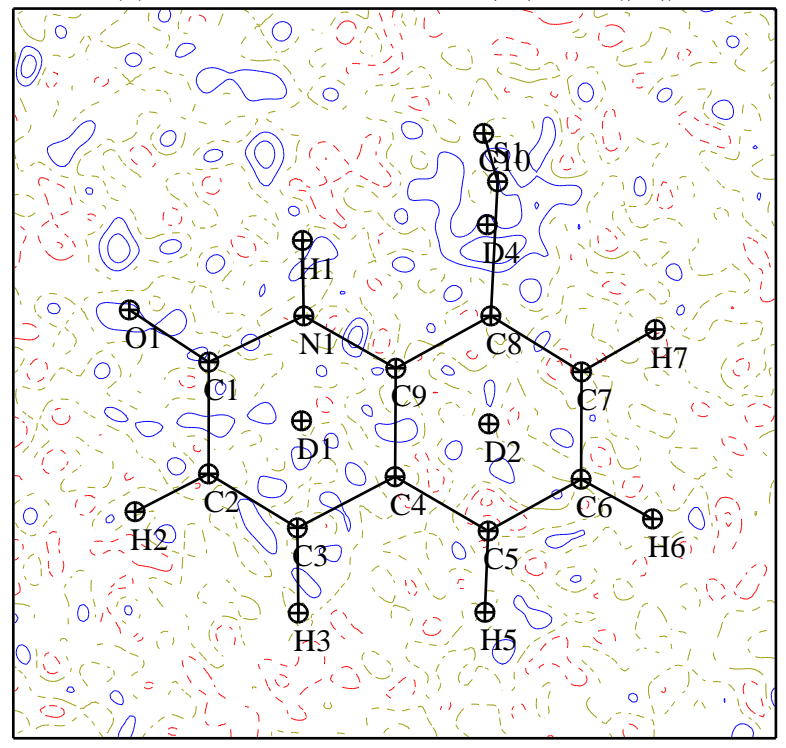

(e) MM, anharmonic $\mathrm{n}=4,|F| \geq 2 \sigma(|F|)$

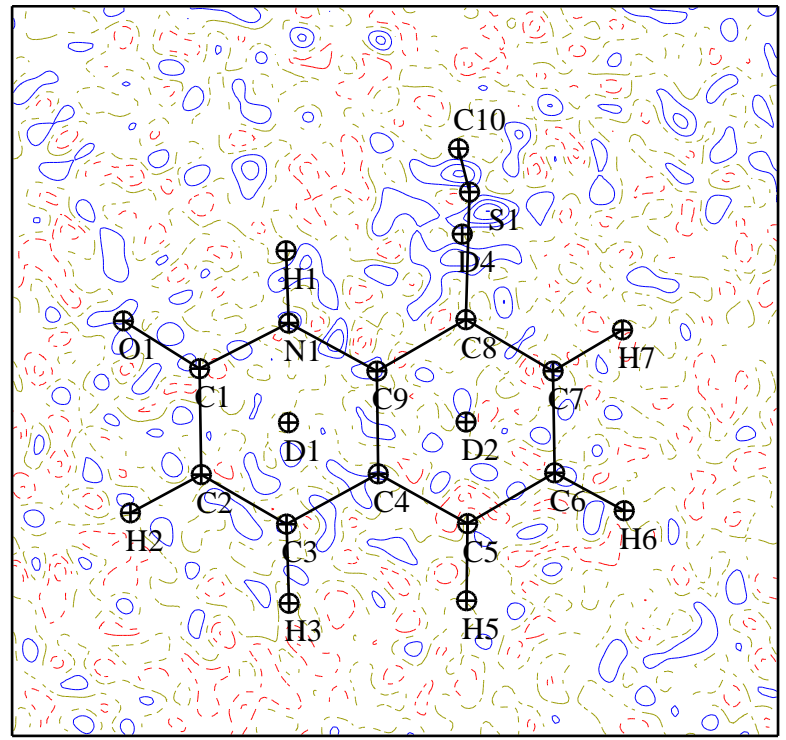

(b) MM, harmonic, all reflections

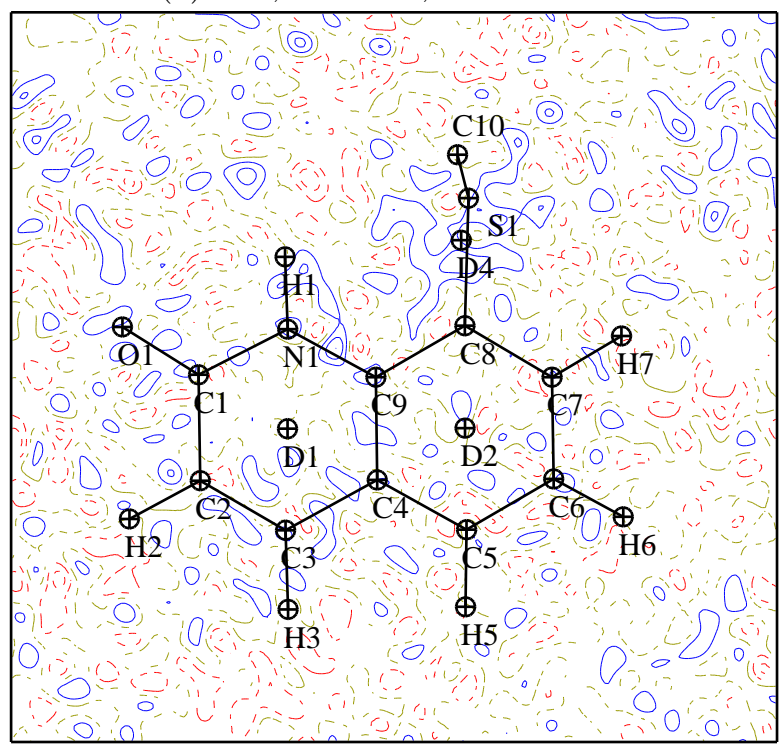

(d) $\mathrm{MM}$, anharmonic $\mathrm{n}=3$, all reflections

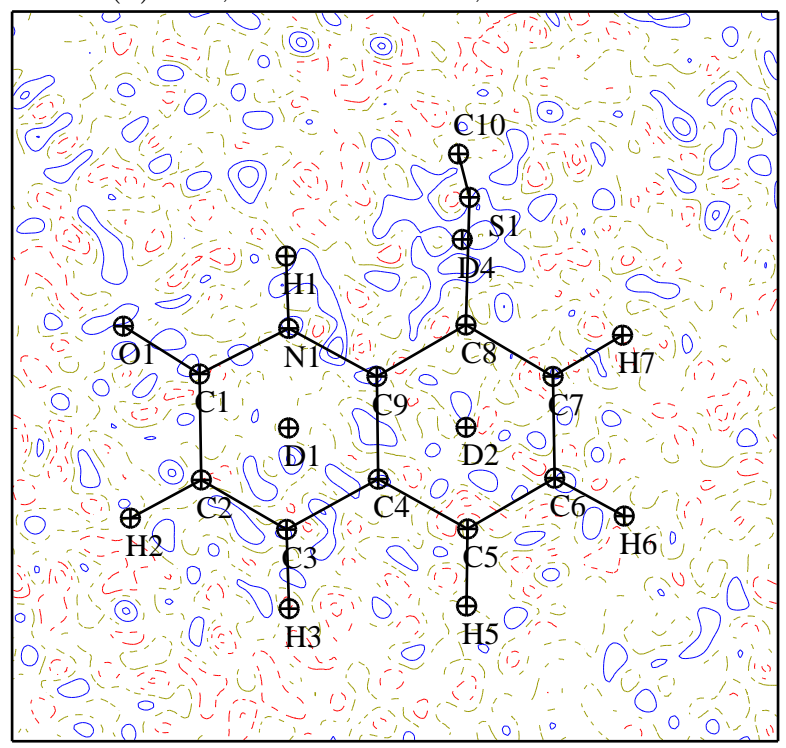

(f) $M M$, anharmonic $\mathrm{n}=4$, all reflections

Figure 18: Residual density maps for PT-11 in the plane of quinoline, MM, unrestrained X-H distances, left: $|F| \geq 2 \sigma(|F|)$ and right: all reflections included. Contour level: $0.05 \mathrm{e} / \AA^{3}$. Colours: blue - positive, red - negative. 


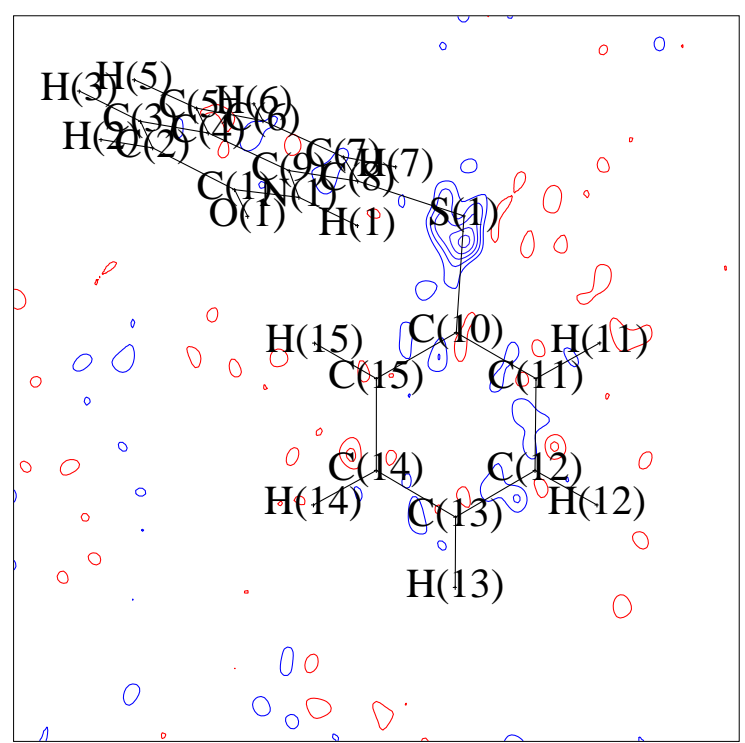

(a) HAR, harmonic, $|F| \geq 2 \sigma(|F|)$

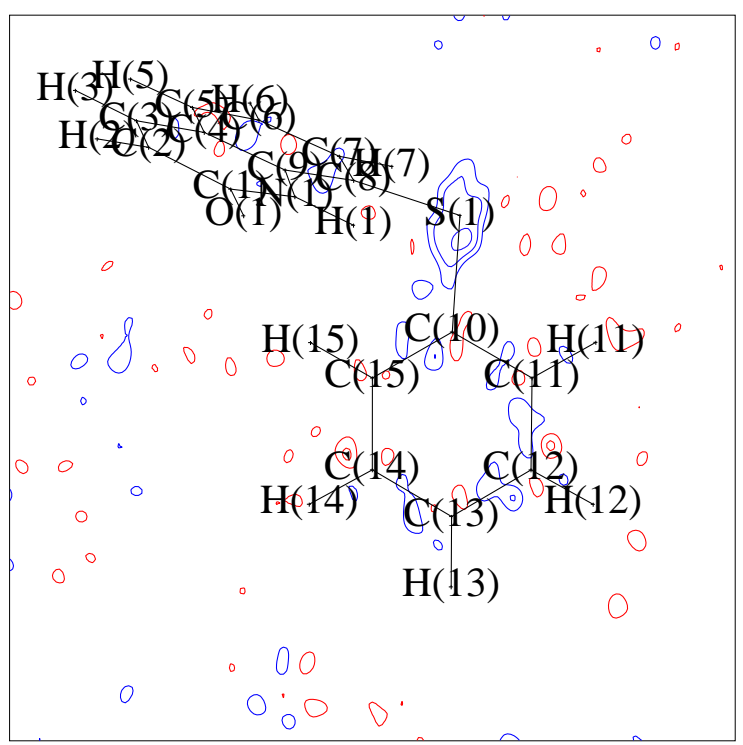

(c) HAR, anharmonic $\mathrm{n}=3,|\widehat{F}| \geq 2 \sigma(|F|)$

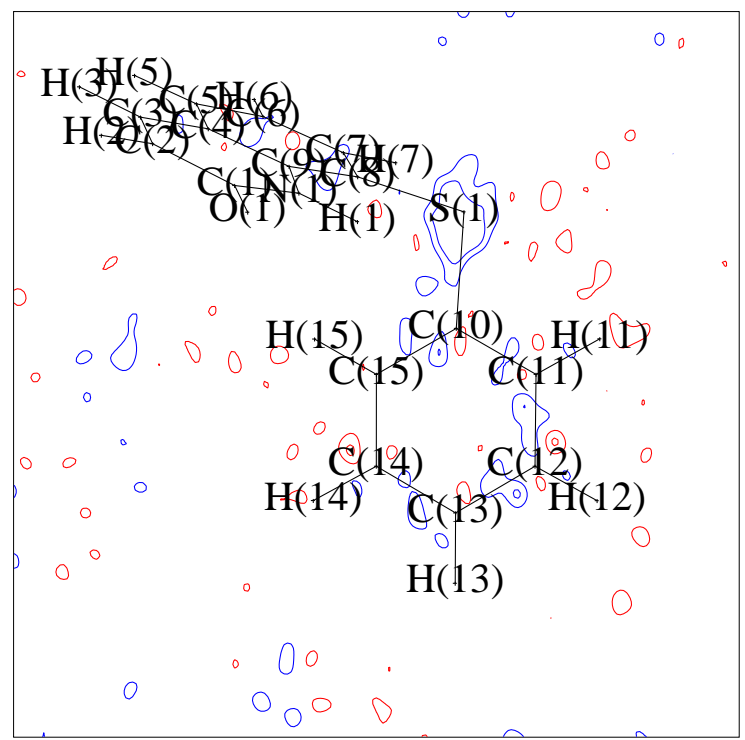

(e) HAR, anharmonic $\mathrm{n}=4,|F| \geq 2 \sigma(|F|)$

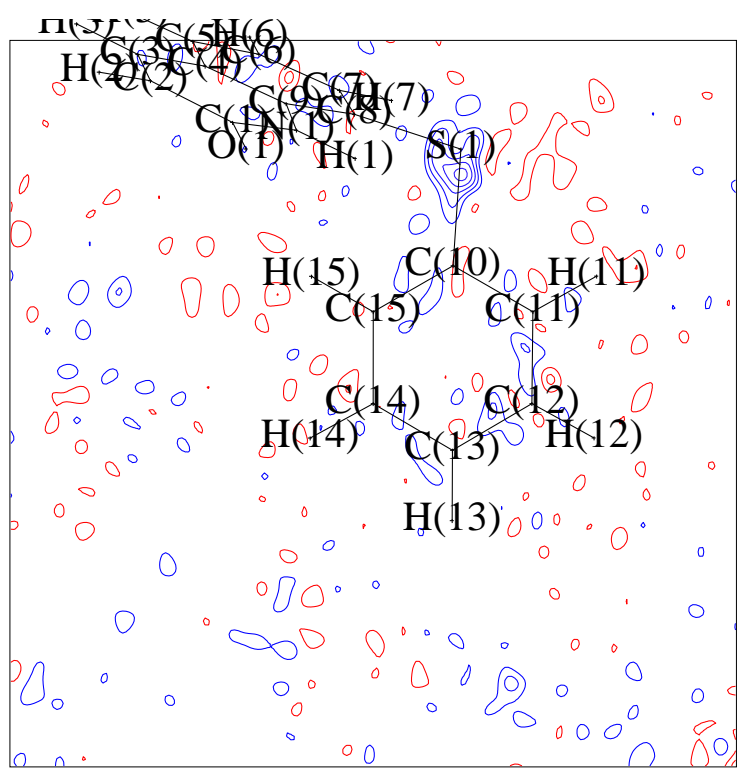

(b) HAR, harmonic, all reflections

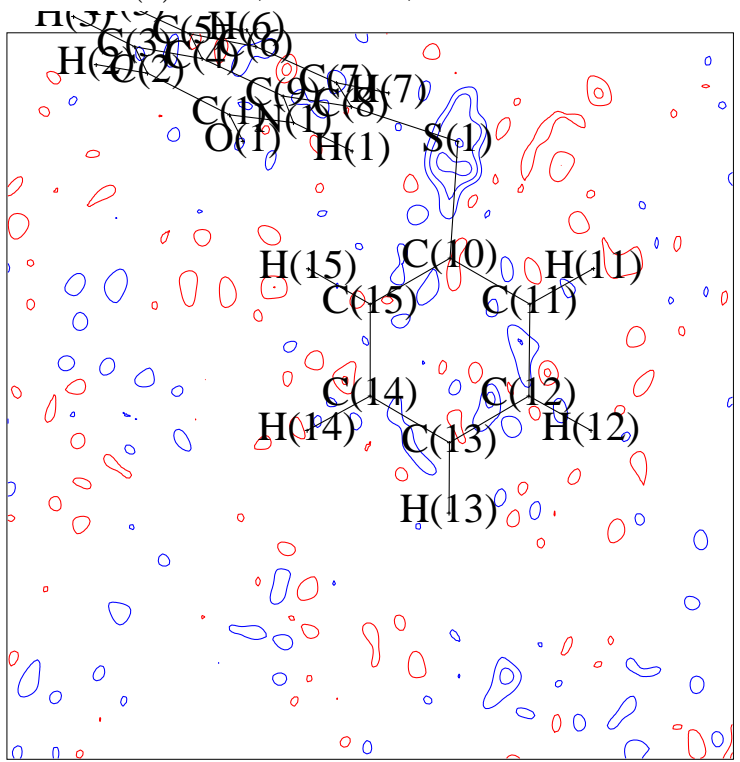

(d) HAR, anharmonic $n=3$, all reflections

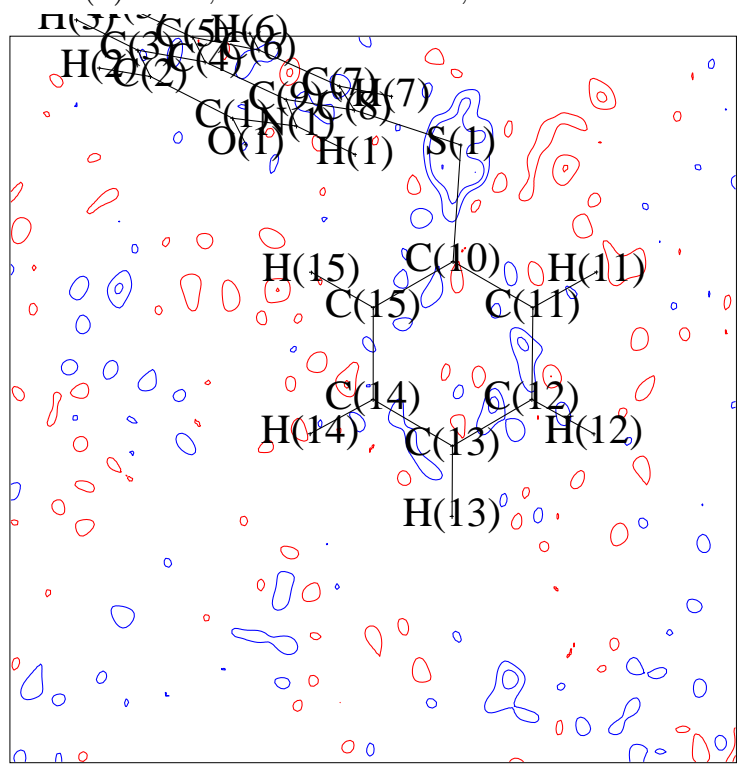

(f) HAR, anharmonic $n=4$, all reflections

Figure 19: Residual density maps for PT-11 in the plane of phenyl ring, HAR, left: $|F| \geq 2 \sigma(|F|)$ and right: all reflections included. Contour level: $0.05 \mathrm{e} / \AA^{3}$. Colours: blue - positive, red - negative. 


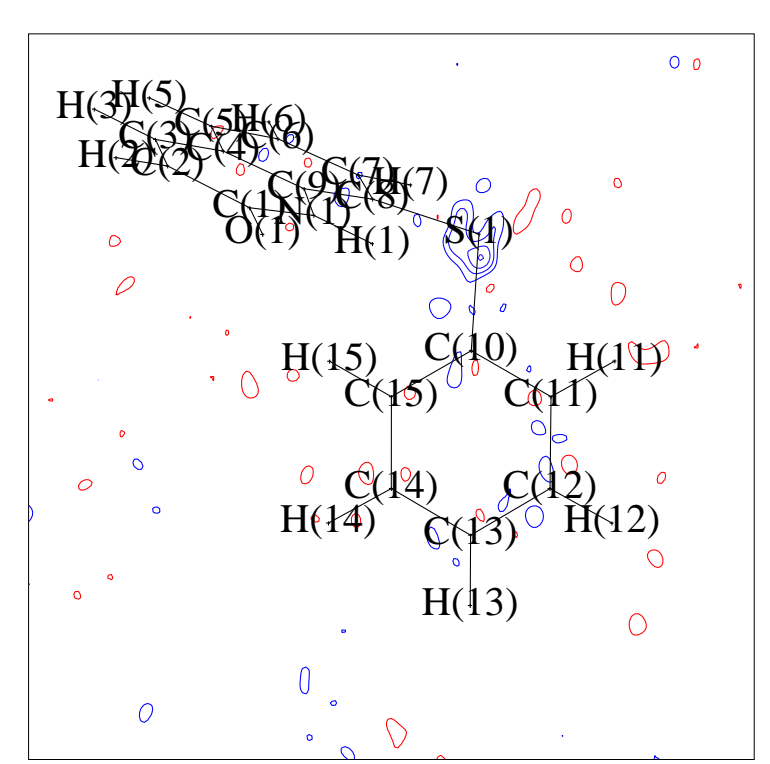

(a) XWR, harmonic, $|F| \geq 2 \sigma(|F|)$

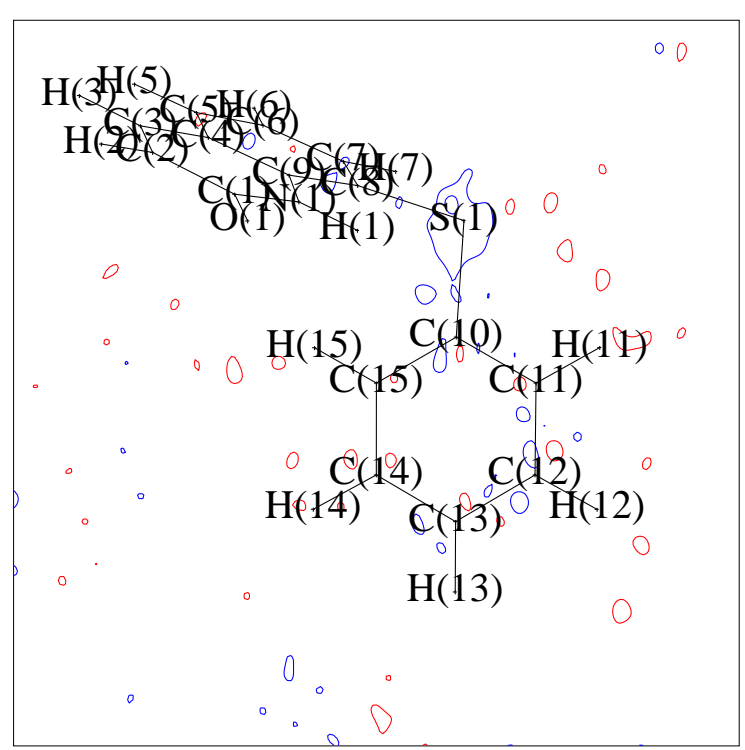

(c) XWR, anharmonic $\mathrm{n}=4,|F| \geq 2 \sigma(|F|)$

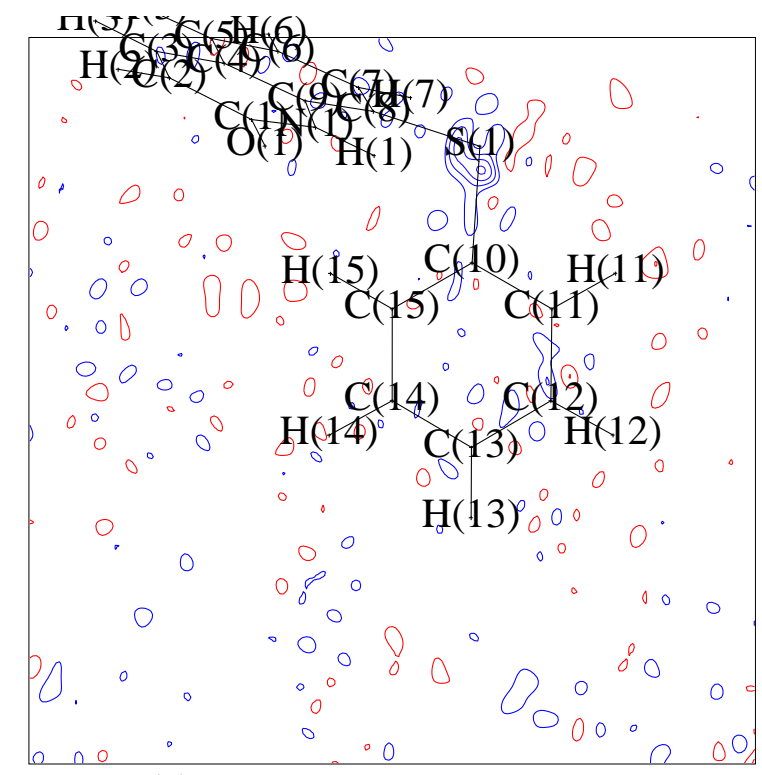

(b) XWR, harmonic, all reflections

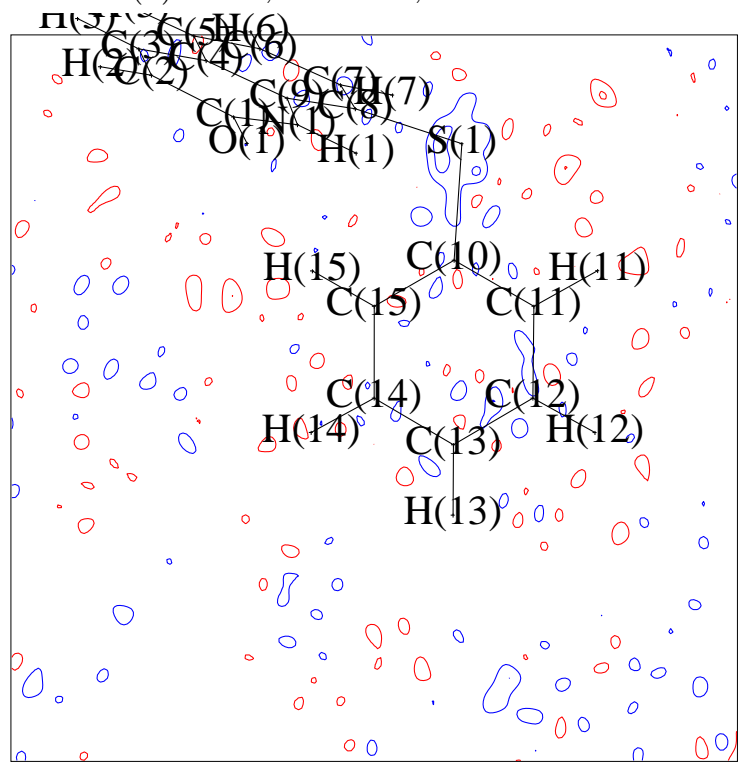

(d) XWR, anharmonic $\mathrm{n}=4$, all reflections

Figure 20: Residual density maps for PT-11 in the plane of phenyl ring, XWR, left: $|F| \geq 2 \sigma(|F|)$ and right: all reflections included. Contour level: $0.05 \mathrm{e} / \AA^{3}$. Colours: blue - positive, red - negative. 


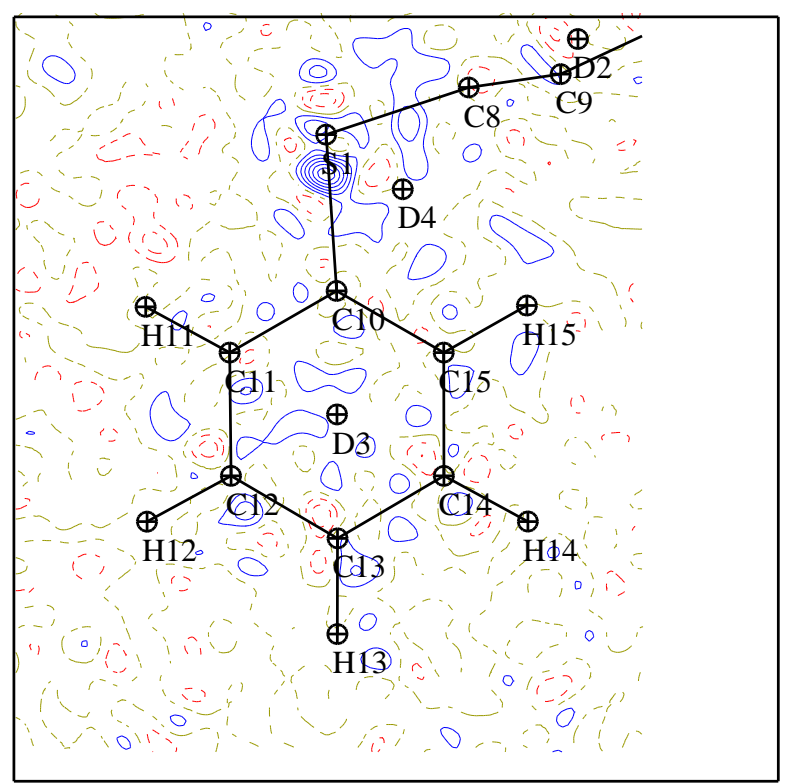

(a) MM, harmonic, $|F| \geq 2 \sigma(|F|)$

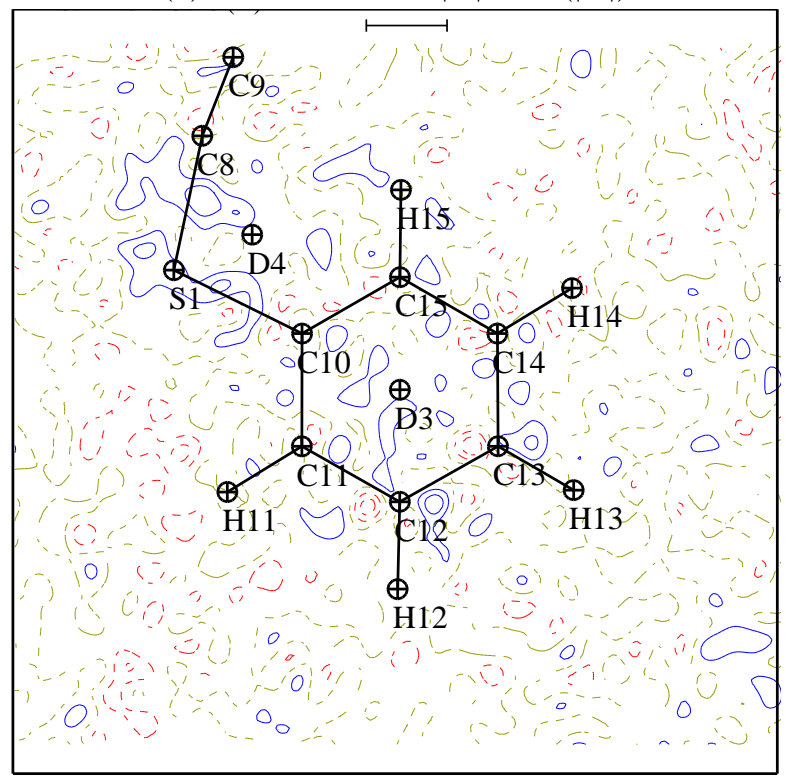

(c) MM, anharmonic $\mathrm{n}=3,|F| \geq 2 \sigma(|F|)$

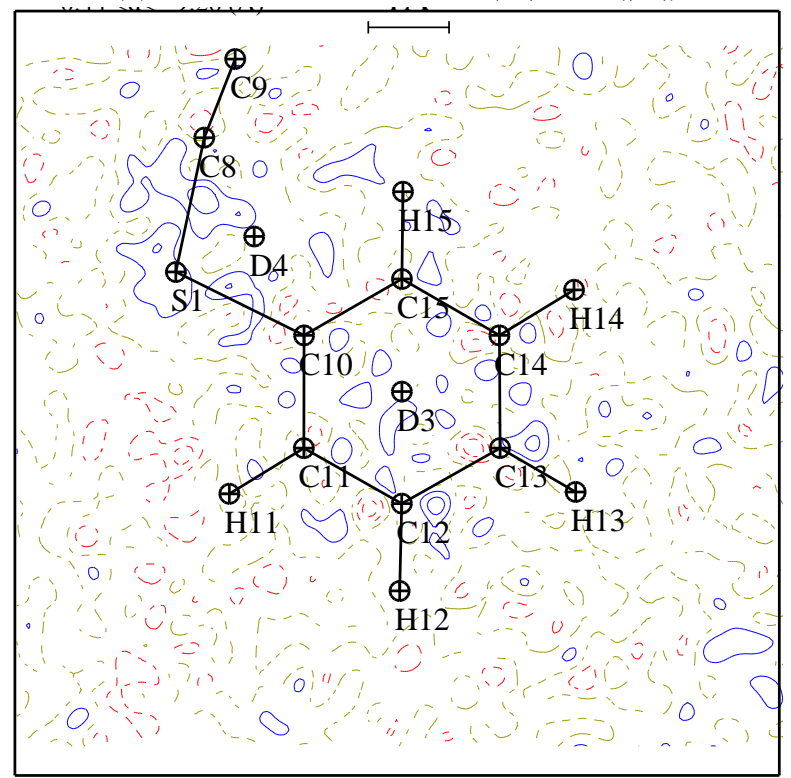

(e) MM, anharmonic $\mathrm{n}=4,|F| \geq 2 \sigma(|F|)$

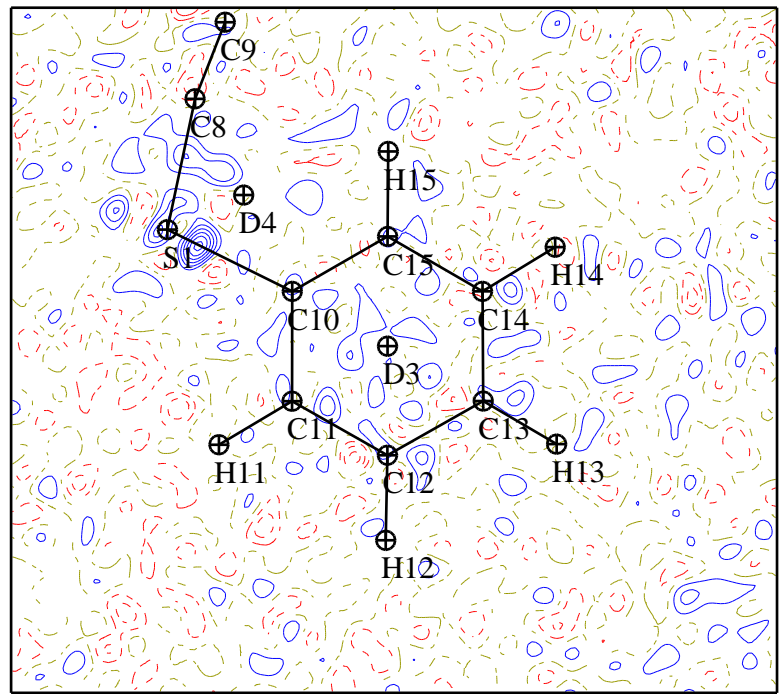

(b) MM, harmonic, all reflections

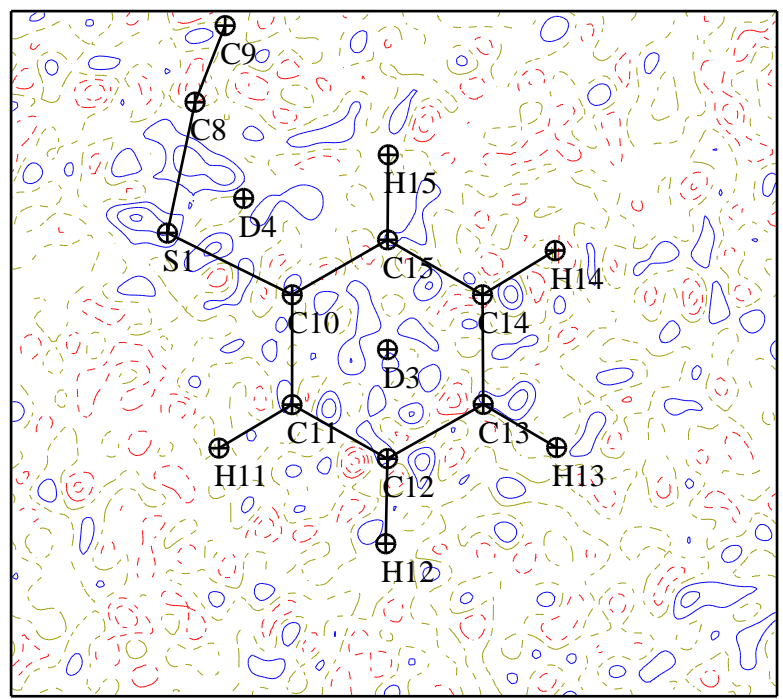

(d) $\mathrm{MM}$, anharmonic $\mathrm{n}=3$, all reflections

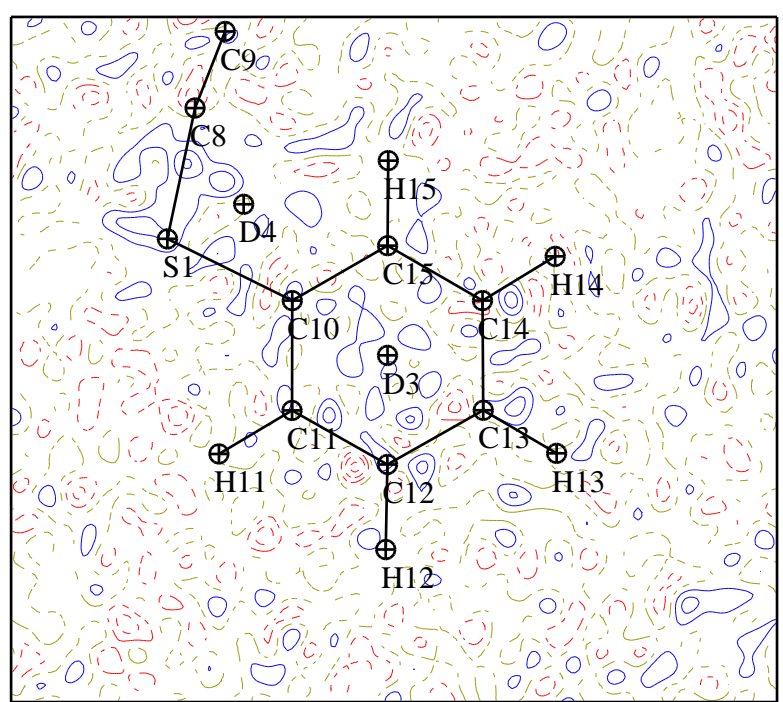

(f) $\mathrm{MM}$, anharmonic $\mathrm{n}=4$, all reflections

Figure 21: Residual density maps for PT-11 in the plane of phenyl ring, MM, restrained X-H distances, left: $|F| \geq 2 \sigma(|F|)$ and right: all reflections included. Contour level: 0.05 e/ $\AA^{3}$. Colours: blue - positive, red - negative. 


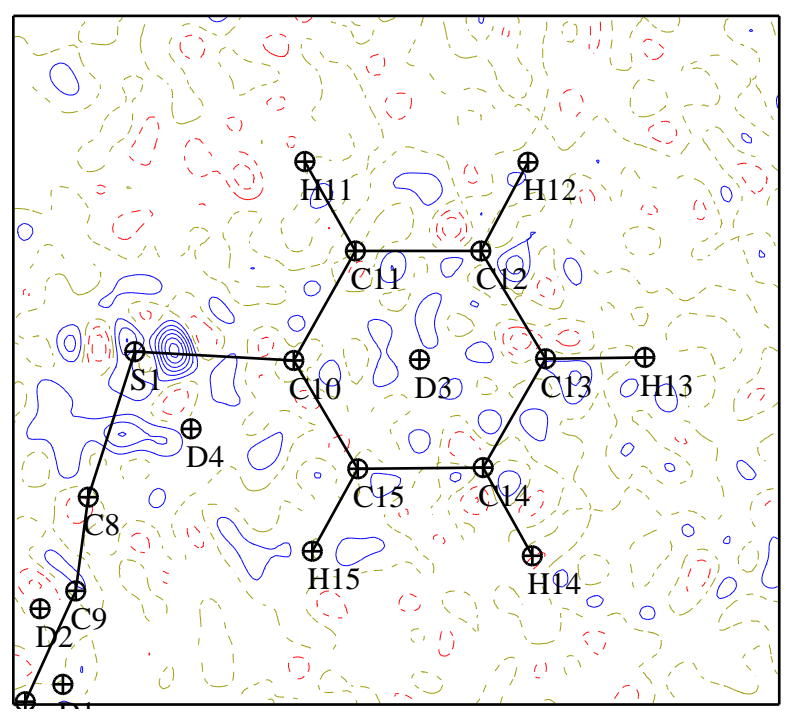

(a) MM, harmonic, $|F| \geq 2 \sigma(|F|)$

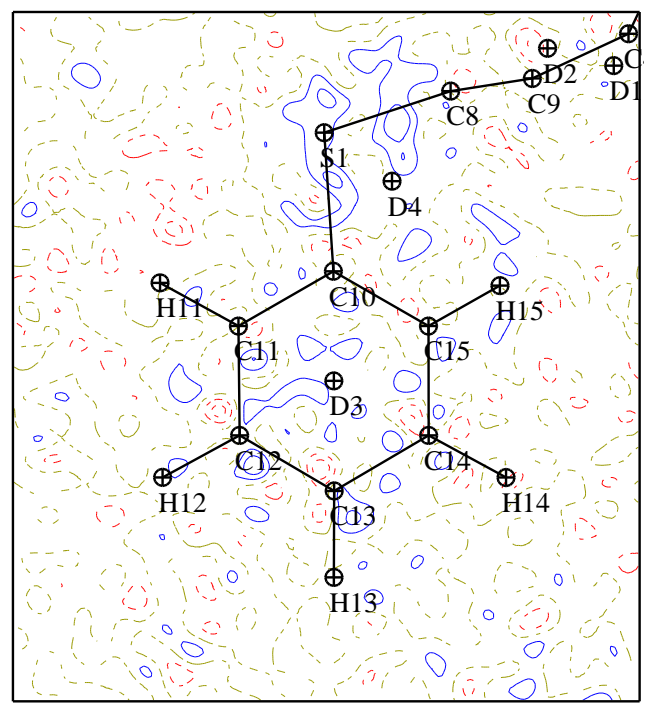

(c) MM, anharmonic n $=3,|F| \geq 2 \sigma(|F|)$

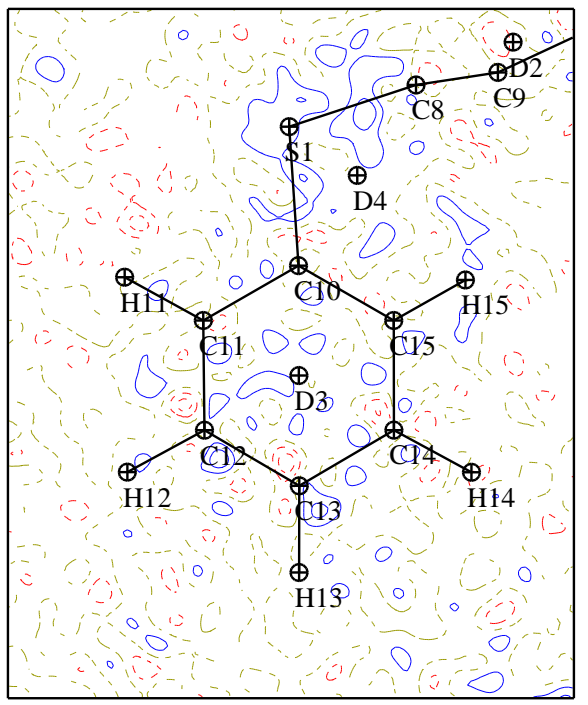

(e) MM, anharmonic $\mathrm{n}=4,|F| \geq 2 \sigma(|F|)$

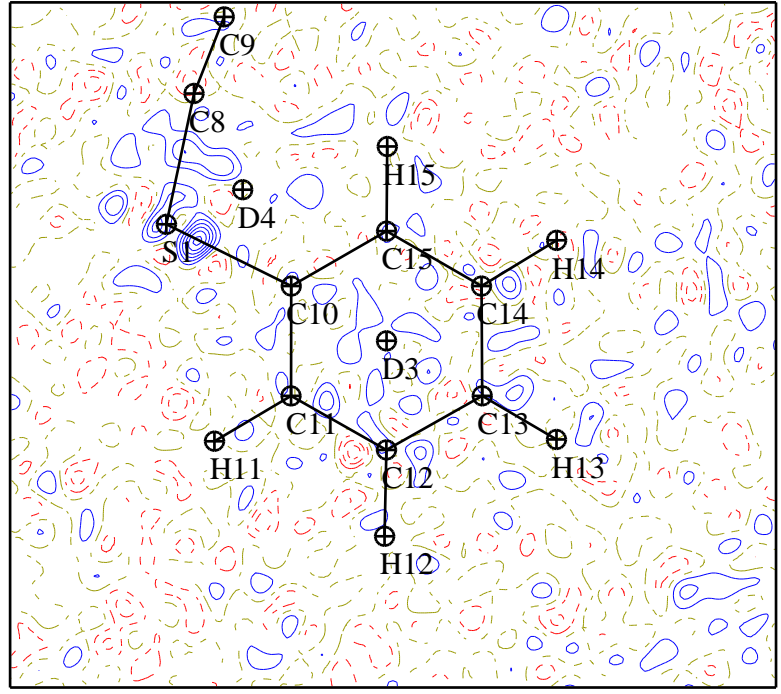

(b) MM, harmonic, all reflections

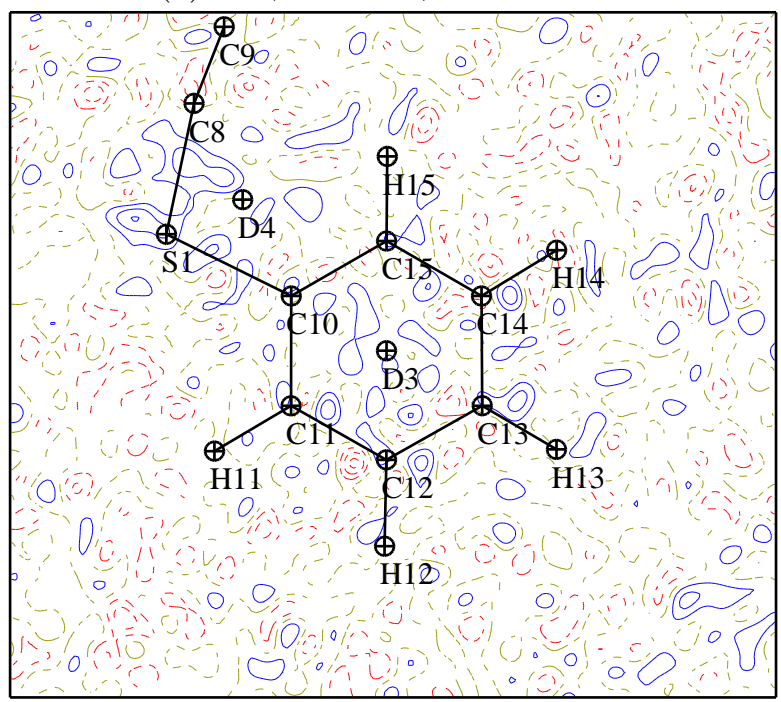

(d) MM, anharmonic $\mathrm{n}=3$, all reflections

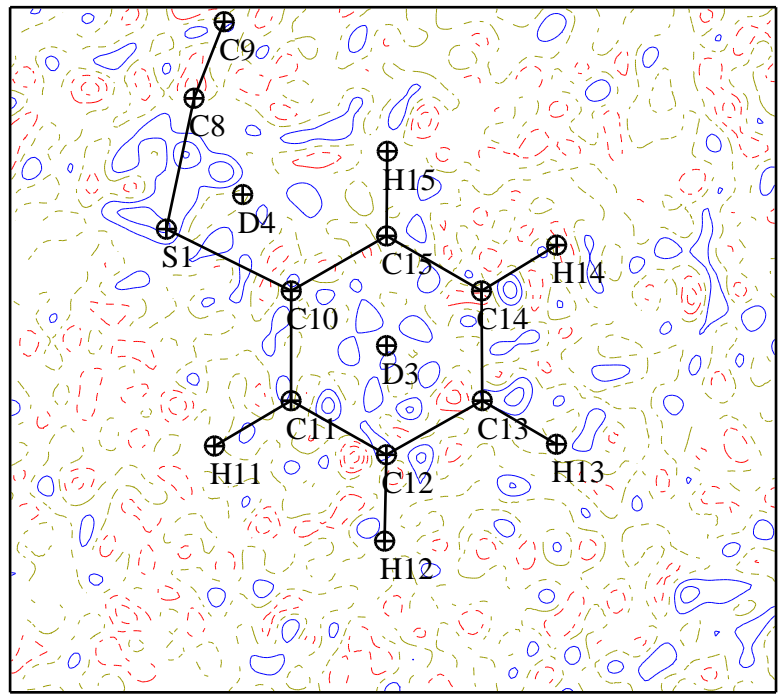

(f) MM, anharmonic $\mathrm{n}=4$, all reflections

Figure 22: Residual density maps for PT-11 in the plane of phenyl ring, MM, unrestrained X-H distances, left: $|F| \geq 2 \sigma(|F|)$ and right: all reflections included. Contour level: $0.05 \mathrm{e} / \AA^{3}$. Colours: blue - positive, red - negative. 


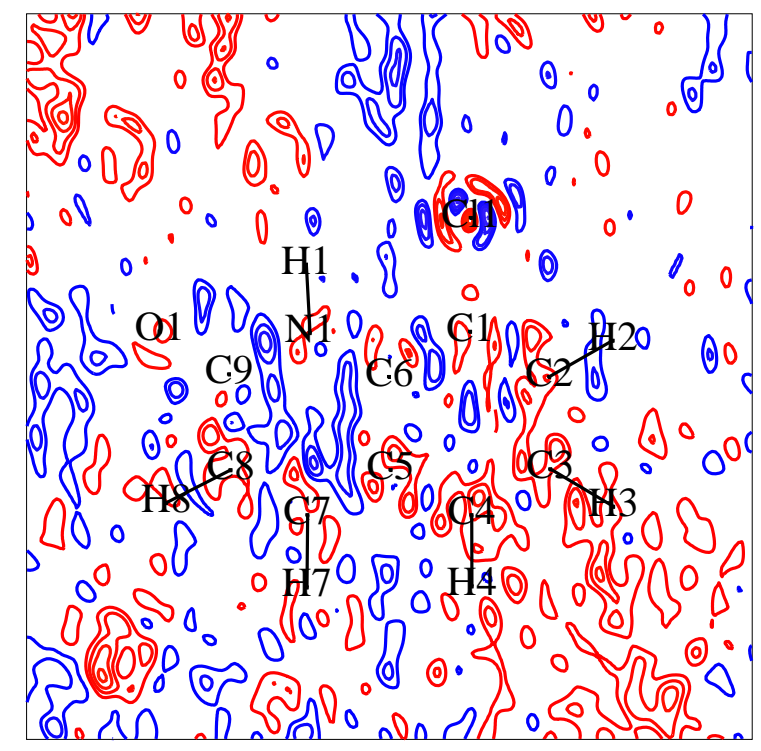

(a) HAR, harmonic, $|F| \geq 2 \sigma(|F|)$

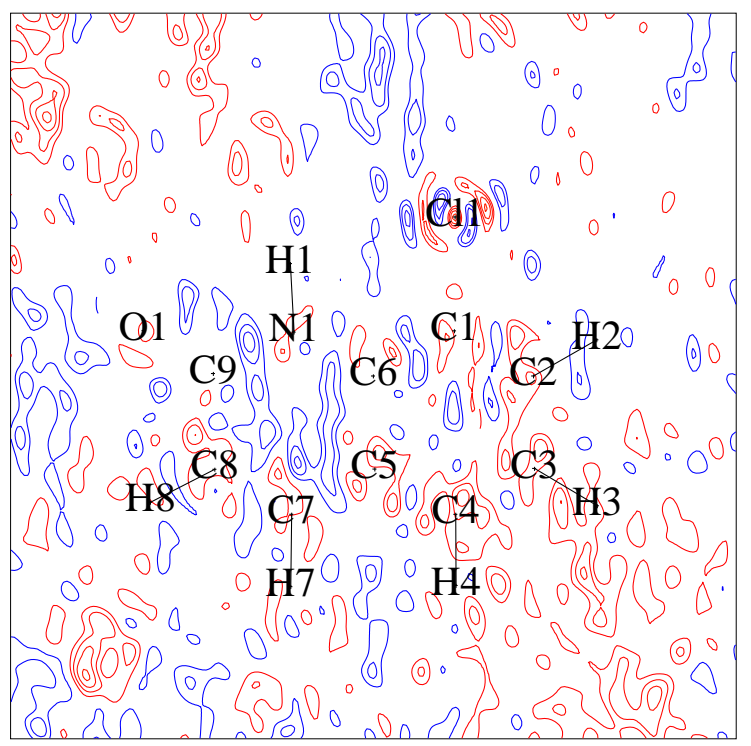

(c) HAR, anharmonic n $=3,|F| \geq 2 \sigma(|F|)$

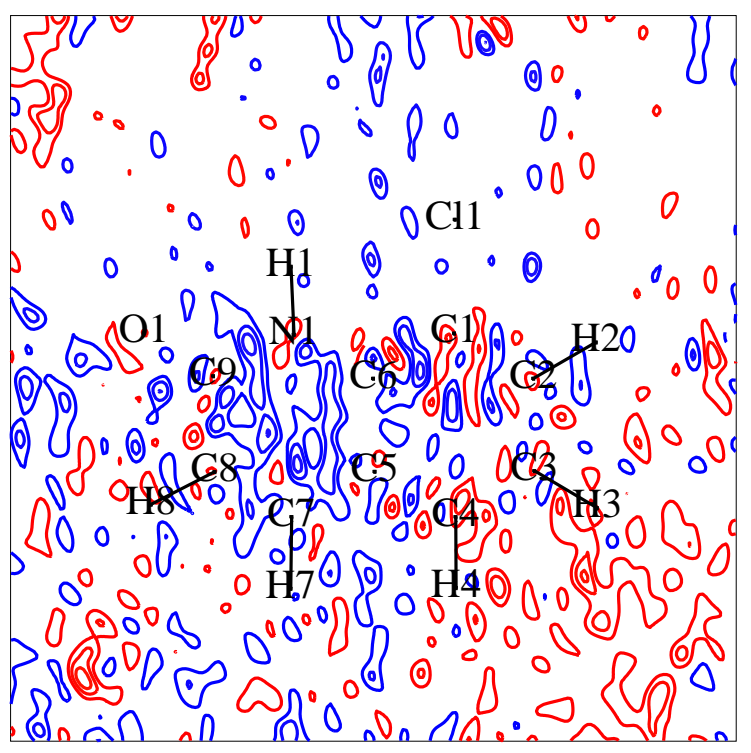

(e) HAR, anharmonic $\mathrm{n}=4,|F| \geq 2 \sigma(|F|)$

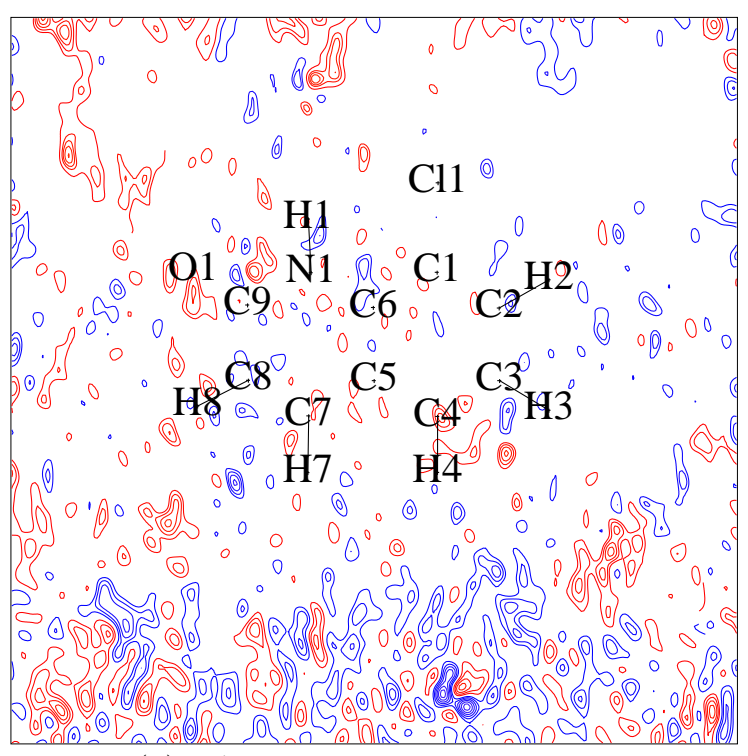

(b) HAR, harmonic, all reflections

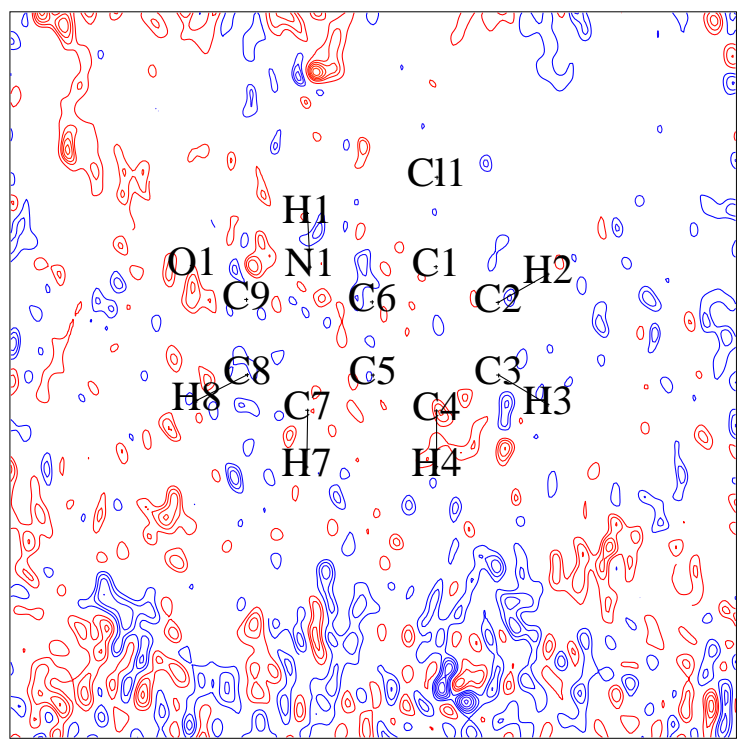

(d) HAR, anharmonic $n=3$, all reflections

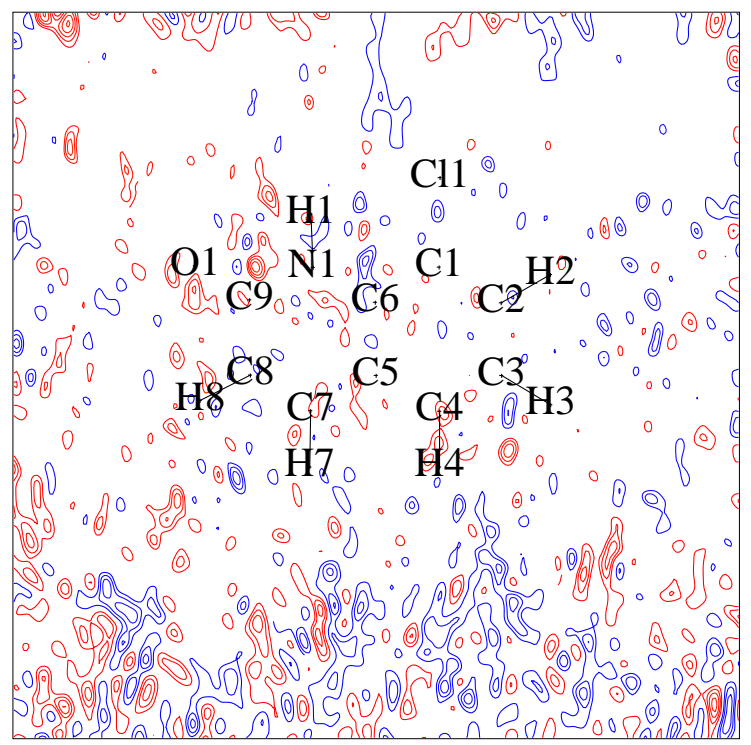

(f) HAR, anharmonic $n=4$, all reflections

Figure 23: Residual density maps for PT-2, HAR, left: $|F| \geq 2 \sigma(|F|)$ and right: all reflections included. Contour level: 0.05 e/ $\AA^{3}$. Colours: blue - positive, red - negative. 


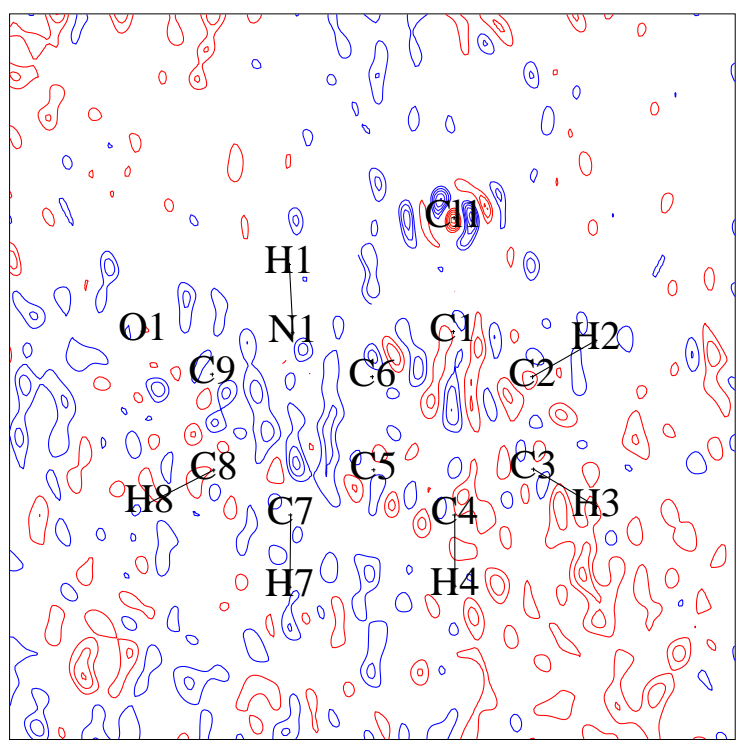

(a) XWR, harmonic, $|F| \geq 2 \sigma(|F|)$

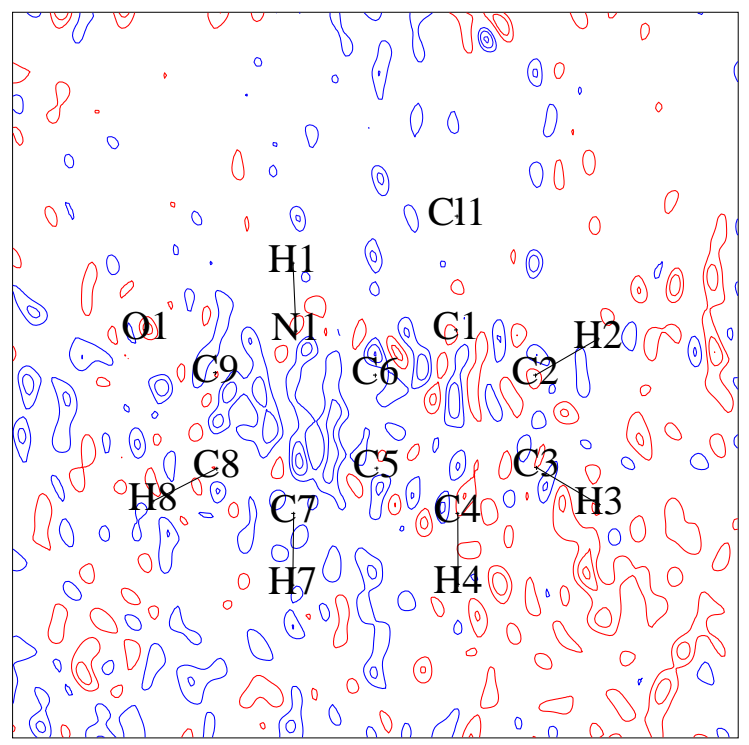

(c) XWR, anharmonic $\mathrm{n}=4,|F| \geq 2 \sigma(|F|)$

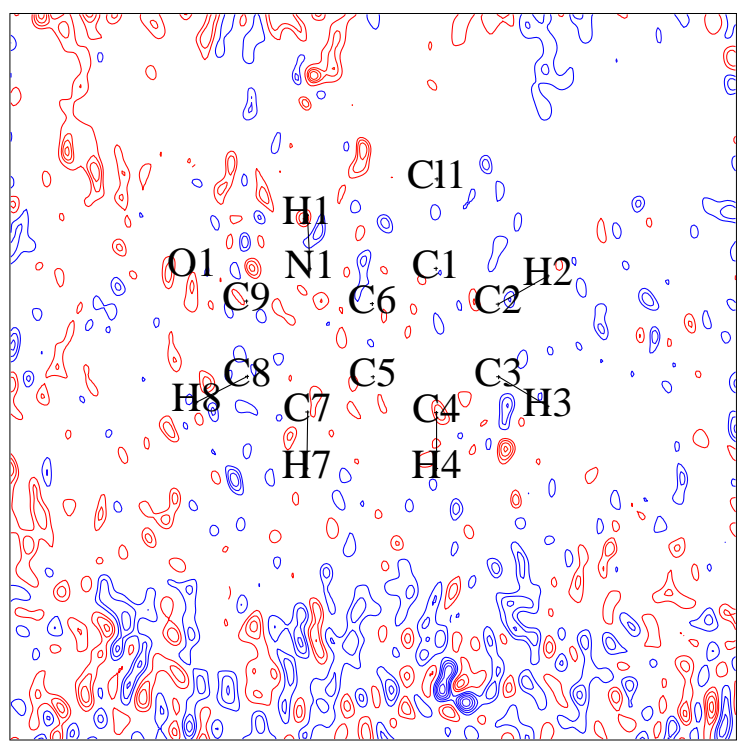

(b) XWR, harmonic, all reflections

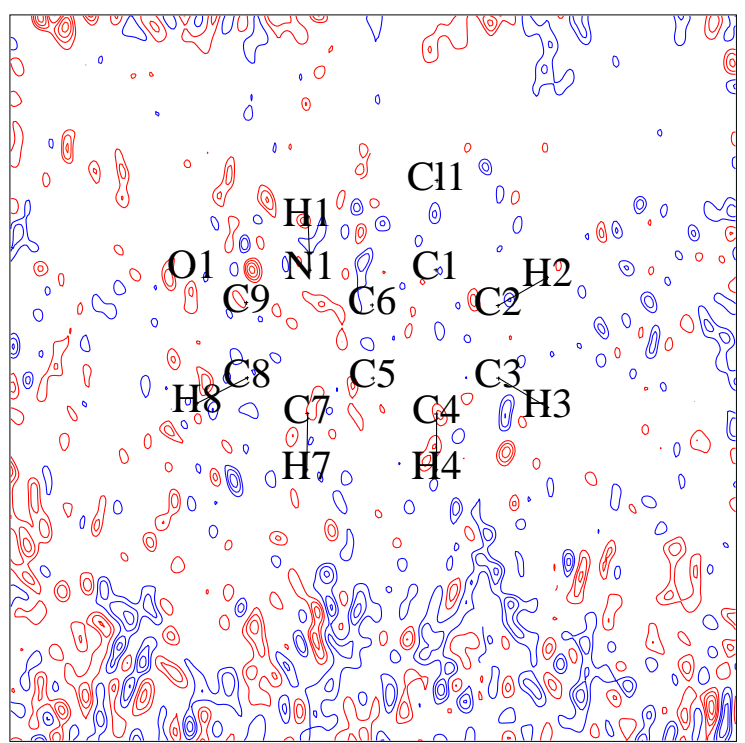

(d) XWR, anharmonic $\mathrm{n}=4$, all reflections

Figure 24: Residual density maps for PT-2, XWR, left: $|F| \geq 2 \sigma(|F|)$ and right: all reflections included. Contour level: 0.05 e/ $\AA^{3}$. Colours: blue - positive, red - negative. 


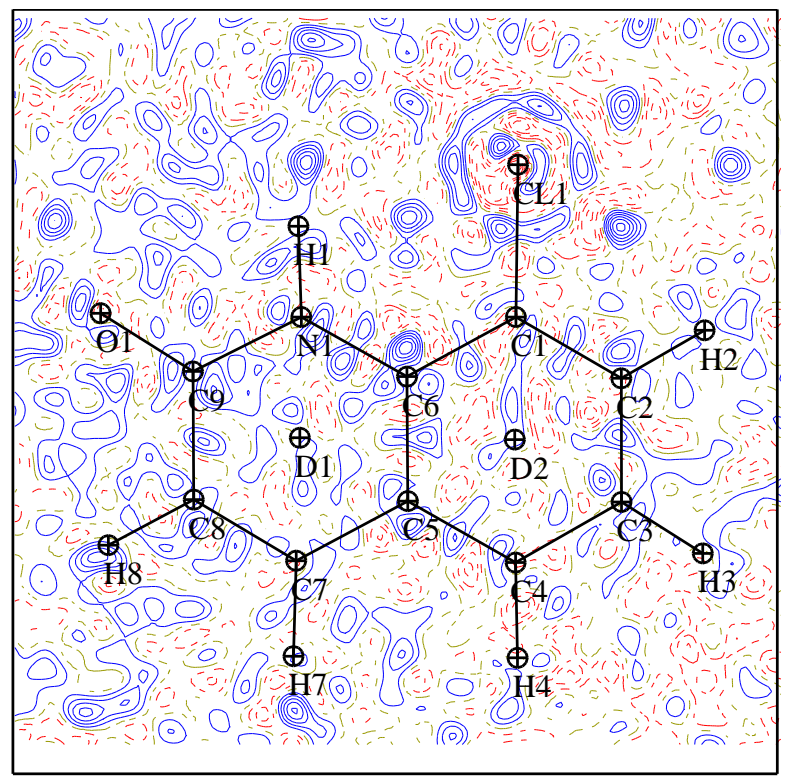

(a) MM, harmonic, $|F| \geq 2 \sigma(|F|)$

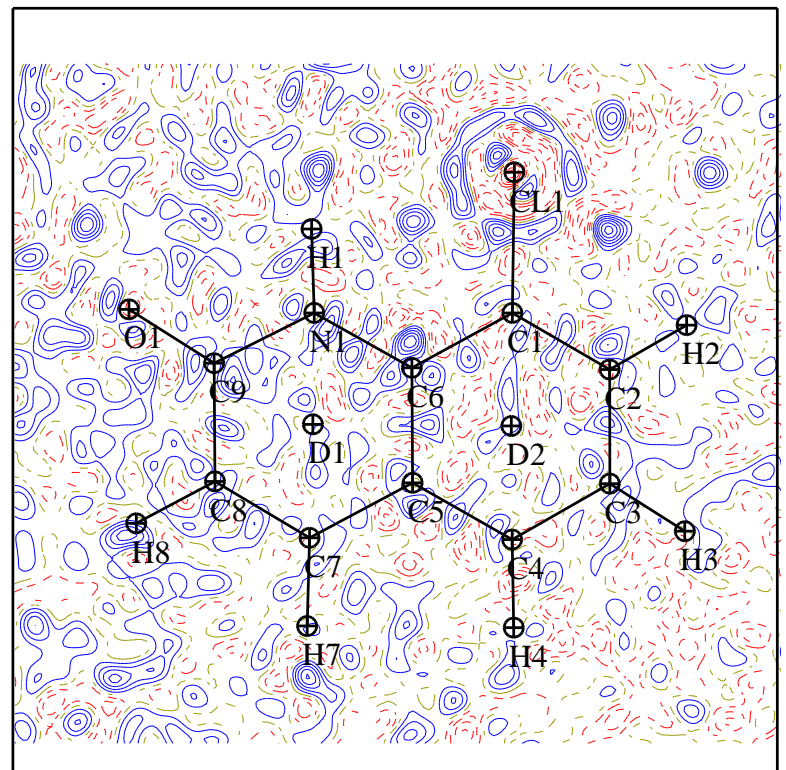

(c) MM, anharmonic $\mathrm{n}=3,|F| \geq 2 \sigma(|F|)$

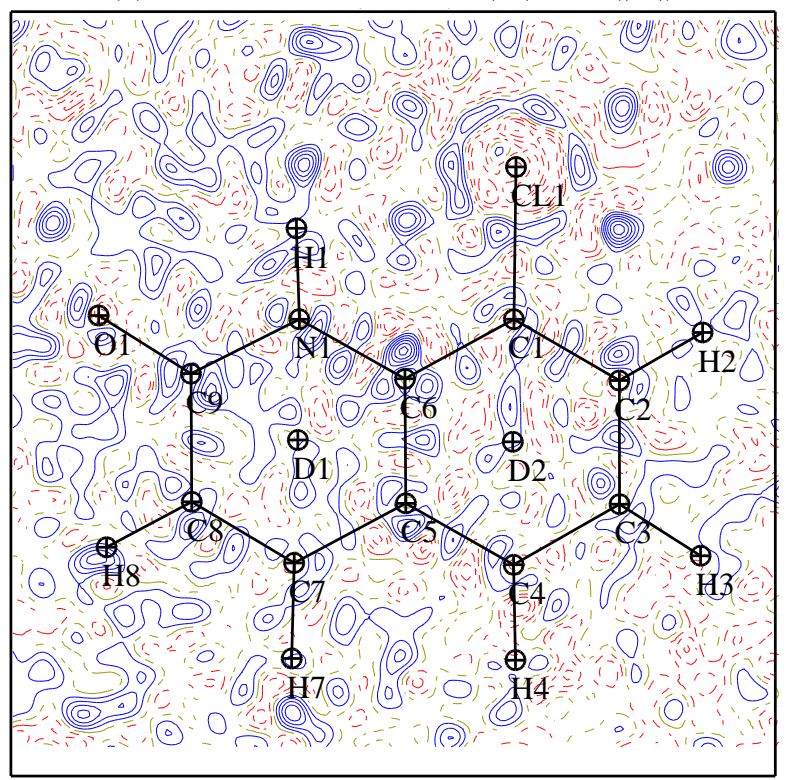

(e) MM, anharmonic $\mathrm{n}=4,|F| \geq 2 \sigma(|F|)$

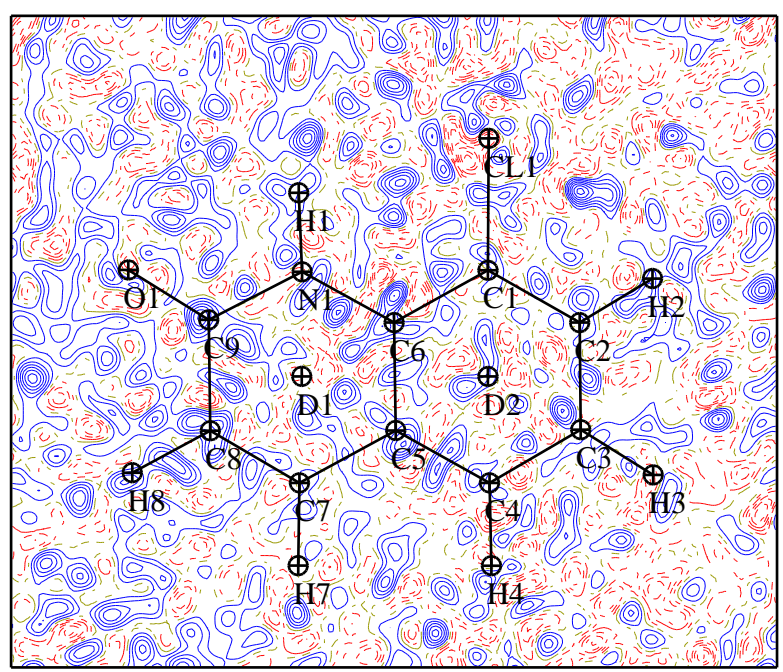

(b) MM, harmonic, all reflections

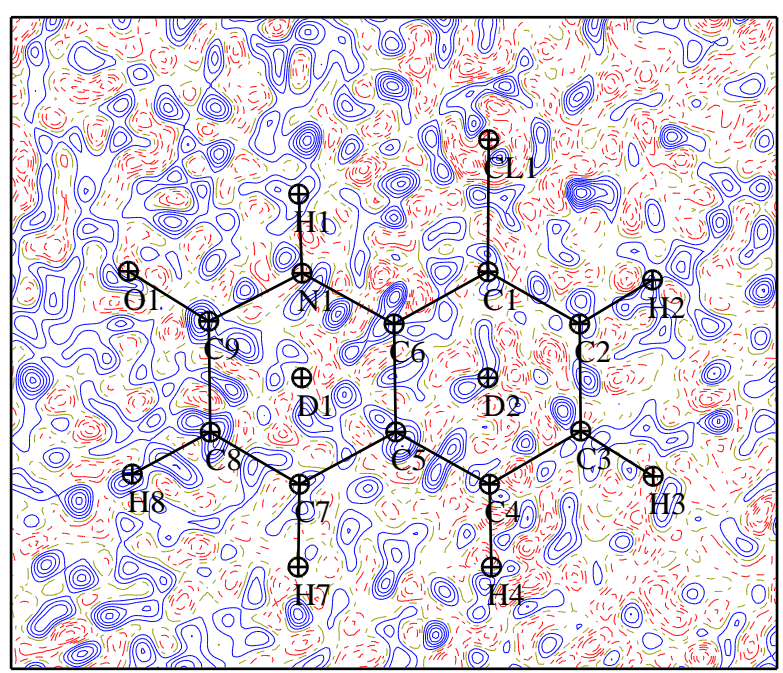

(d) $\mathrm{MM}$, anharmonic $\mathrm{n}=3$, all reflections

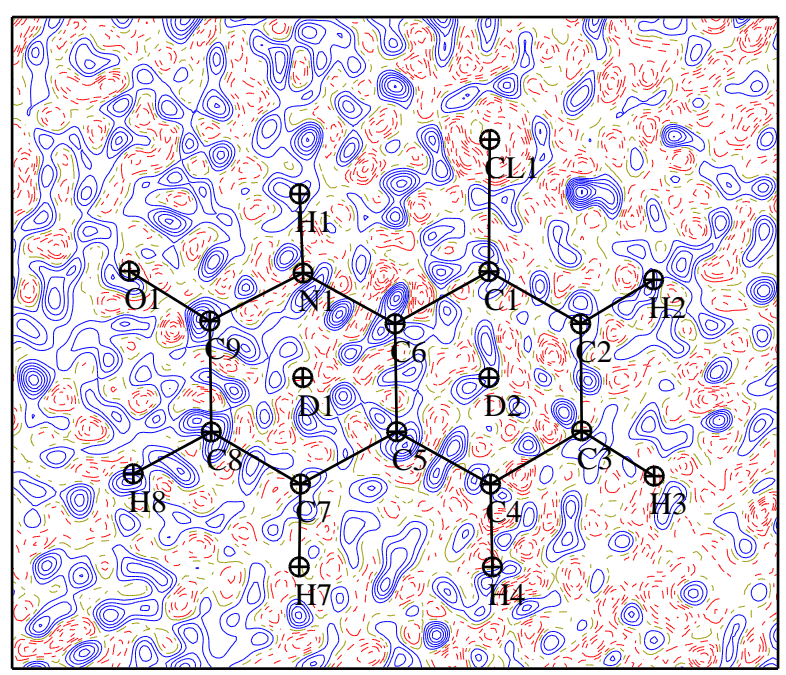

(f) $M M$, anharmonic $n=4$, all reflections

Figure 25: Residual density maps for PT-2, MM, restrained X-H distances, left: $|F| \geq 2 \sigma(|F|)$ and right: all reflections included. Contour level: $0.05 \mathrm{e} / \AA^{3}$. Colours: blue - positive, red - negative. 


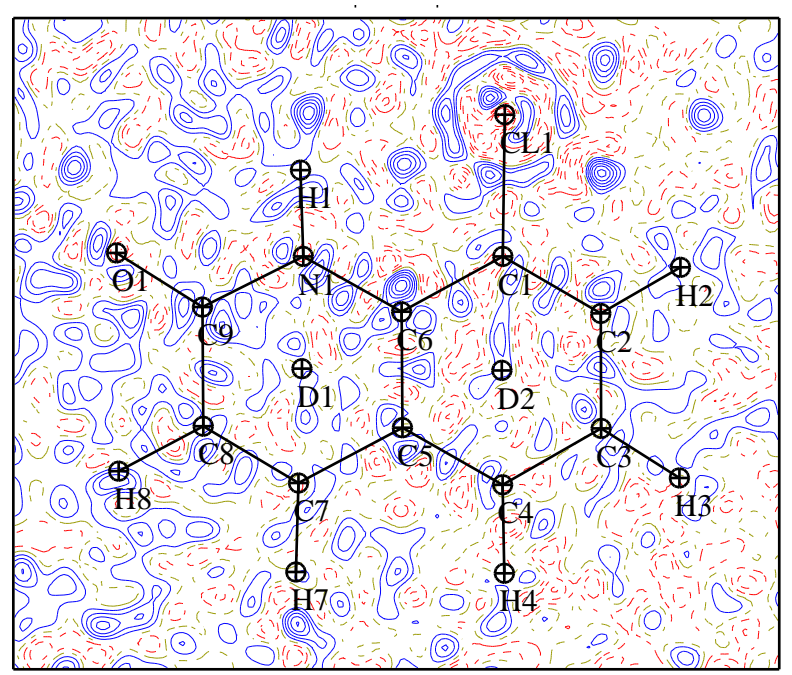

(a) MM, harmonic, $|F| \geq 2 \sigma(|F|)$

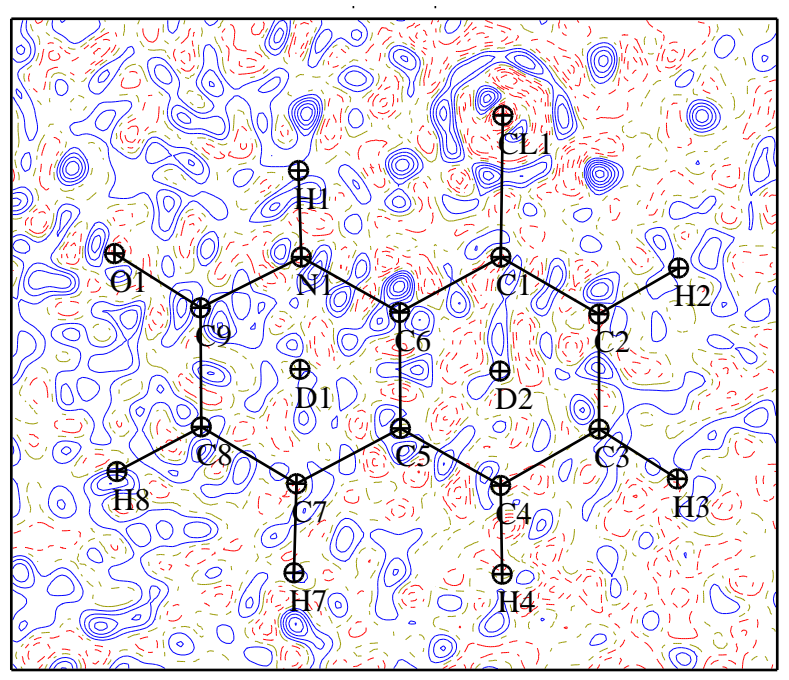

(c) MM, anharmonic n $=3,|F| \geq 2 \sigma(|F|)$

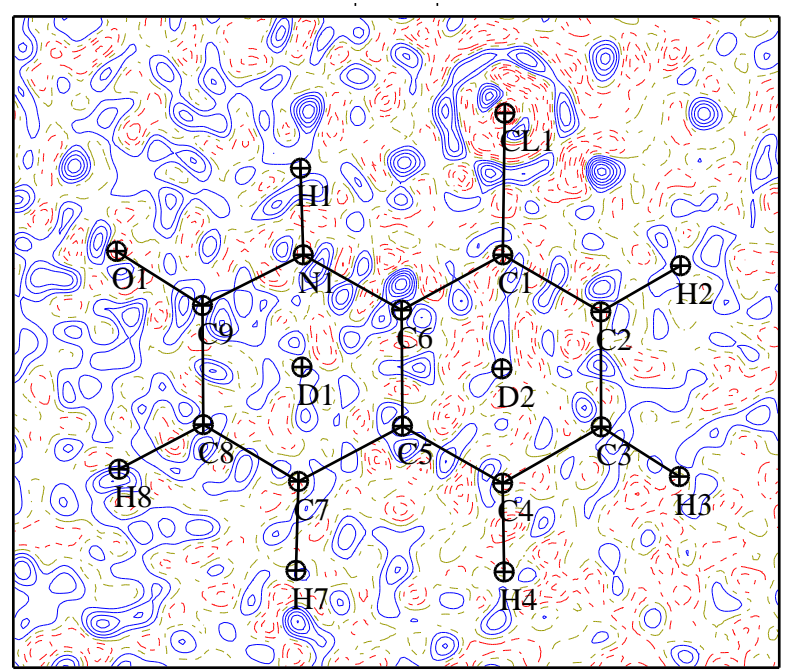

(e) MM, anharmonic $\mathrm{n}=4,|F| \geq 2 \sigma(|F|)$

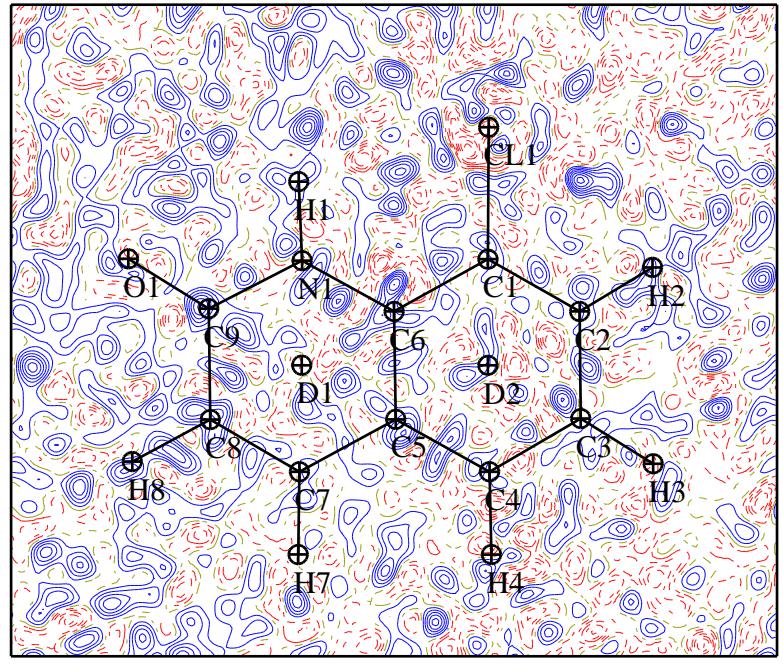

(b) MM, harmonic, all reflections

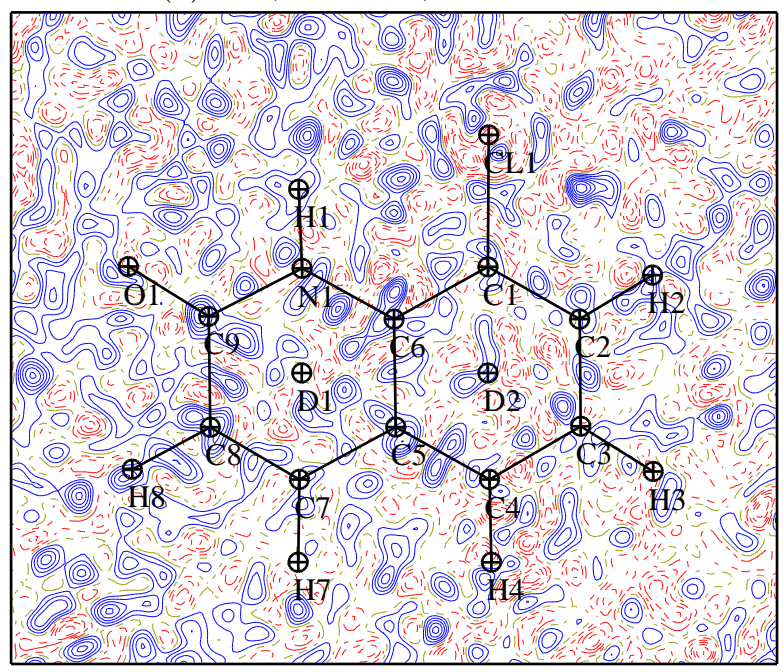

(d) MM, anharmonic $\mathrm{n}=3$, all reflections

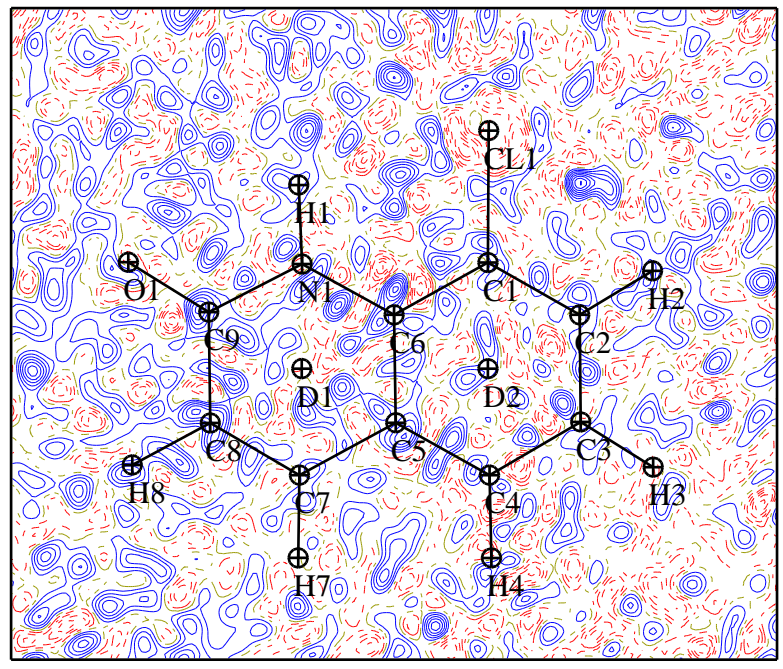

(f) MM, anharmonic $\mathrm{n}=4$, all reflections

Figure 26: Residual density maps for PT-2, MM, unrestrained X-H distances, left: $|F| \geq 2 \sigma(|F|)$ and right: all reflections included. Contour level: $0.05 \mathrm{e} / \AA^{3}$. Colours: blue - positive, red - negative. 


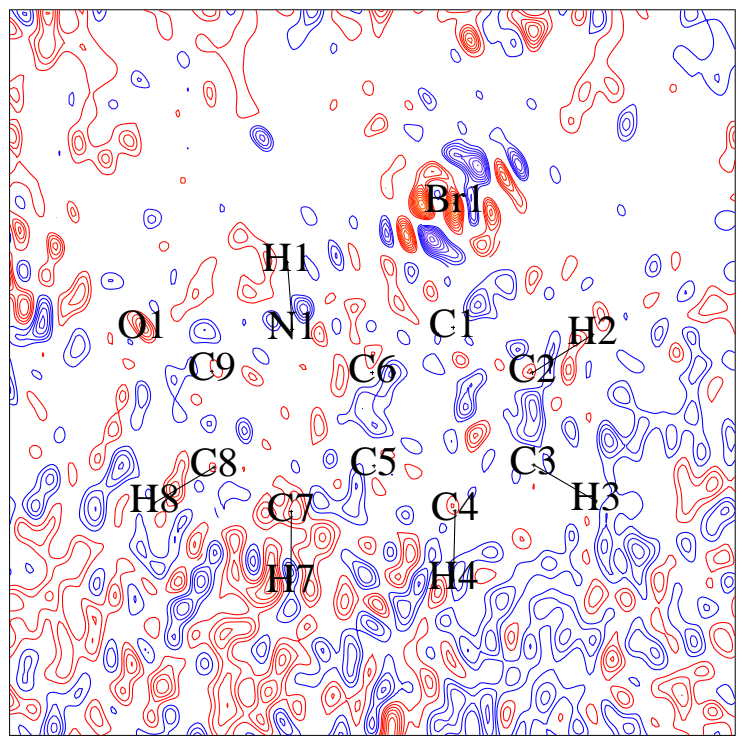

(a) HAR, harmonic, $|F| \geq 2 \sigma(|F|)$

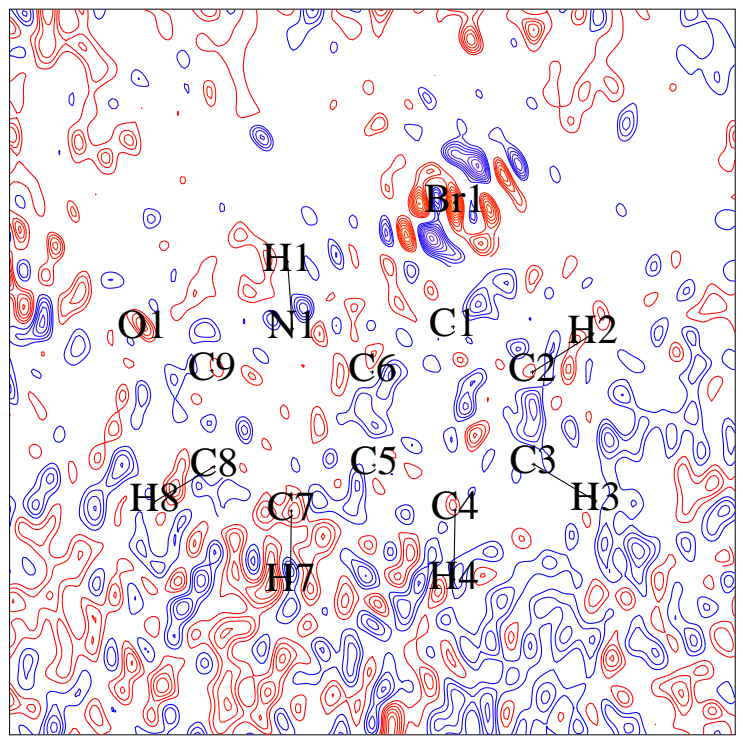

(c) HAR, anharmonic $\mathrm{n}=3,|F| \geq 2 \sigma(|F|)$

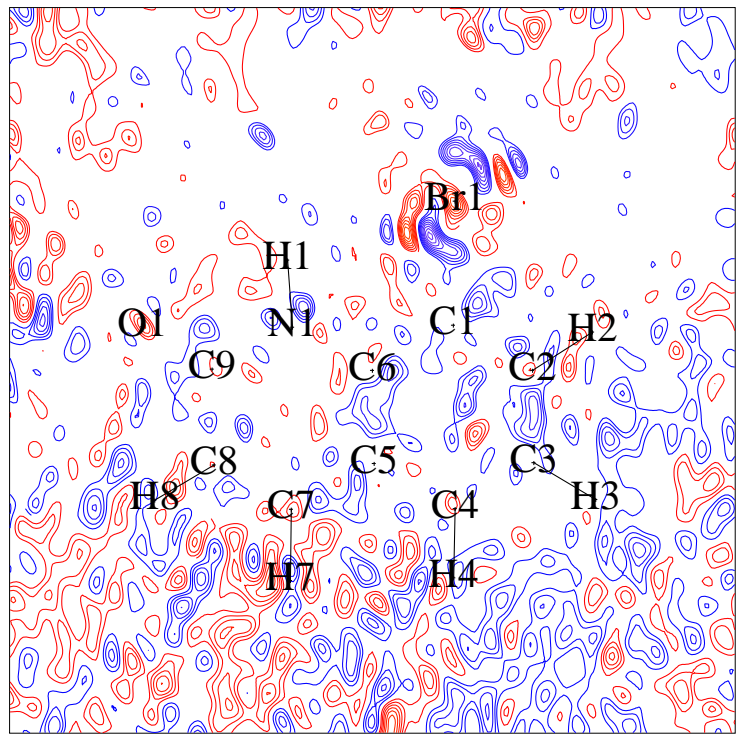

(e) HAR, anharmonic $\mathrm{n}=4,|F| \geq 2 \sigma(|F|)$

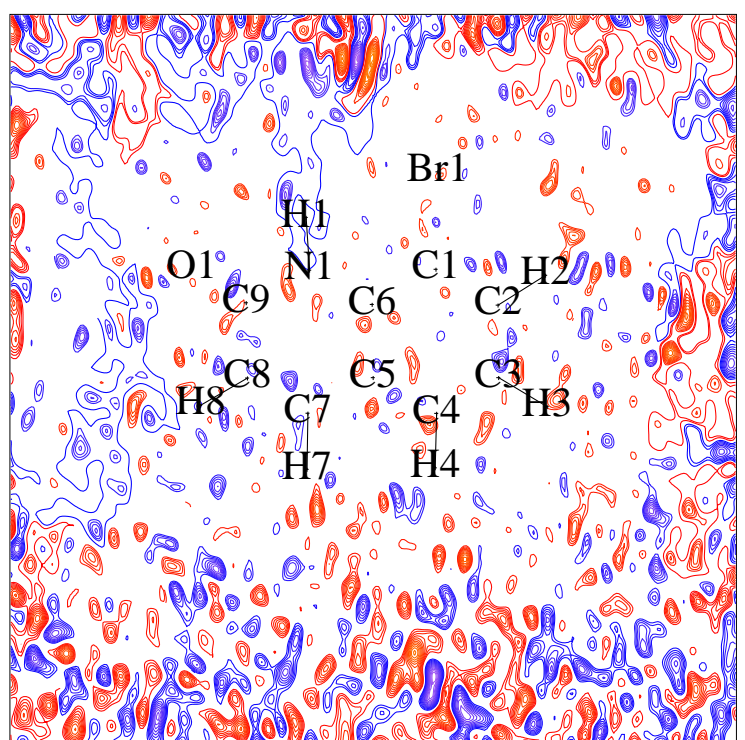

(b) HAR, harmonic, all reflections

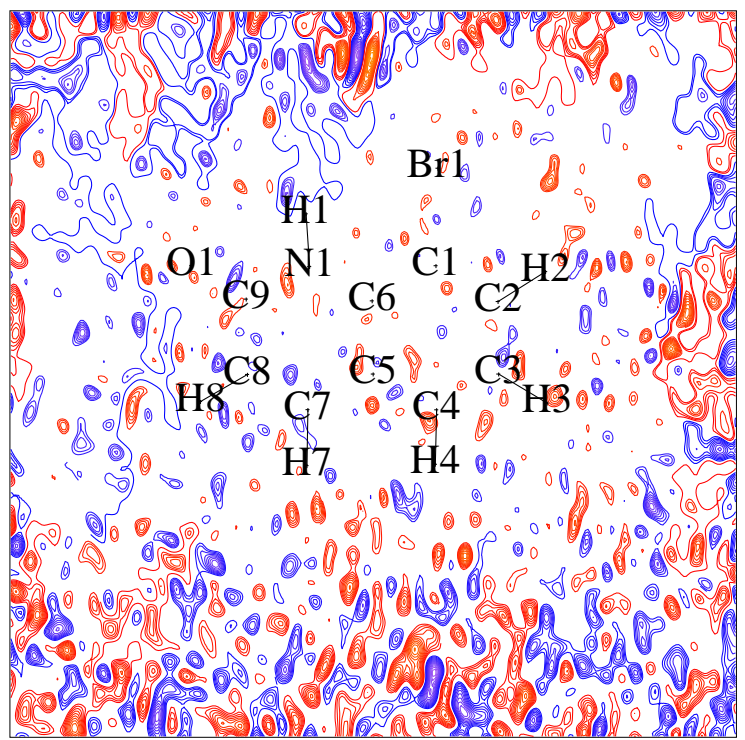

(d) HAR, anharmonic $n=3$, all reflections

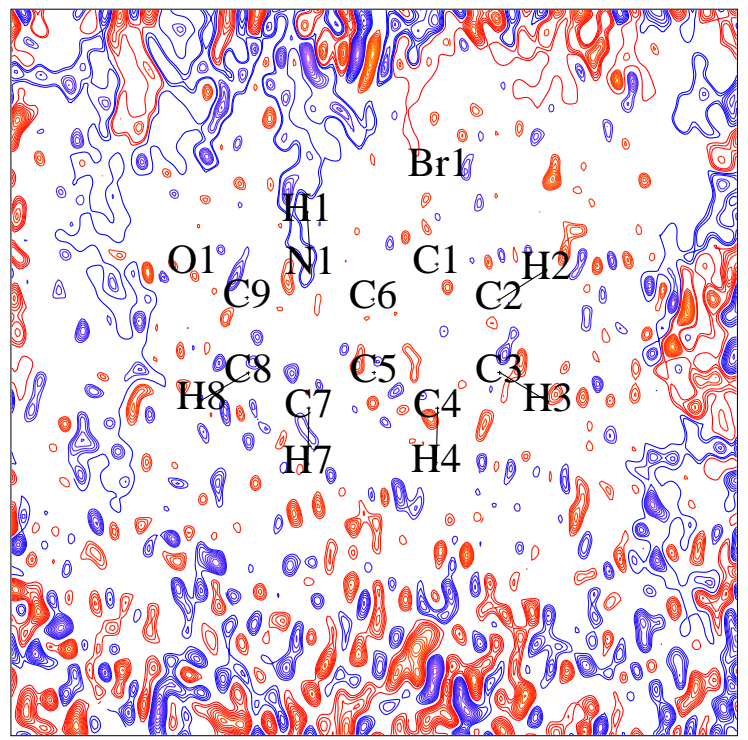

(f) HAR, anharmonic $n=4$, all reflections

Figure 27: Residual density maps for PT-8, HAR, left: $|F| \geq 2 \sigma(|F|)$ and right: all reflections included. Contour level: 0.05 $\mathrm{e} / \AA^{3}$. Colours: blue - positive, red - negative. 


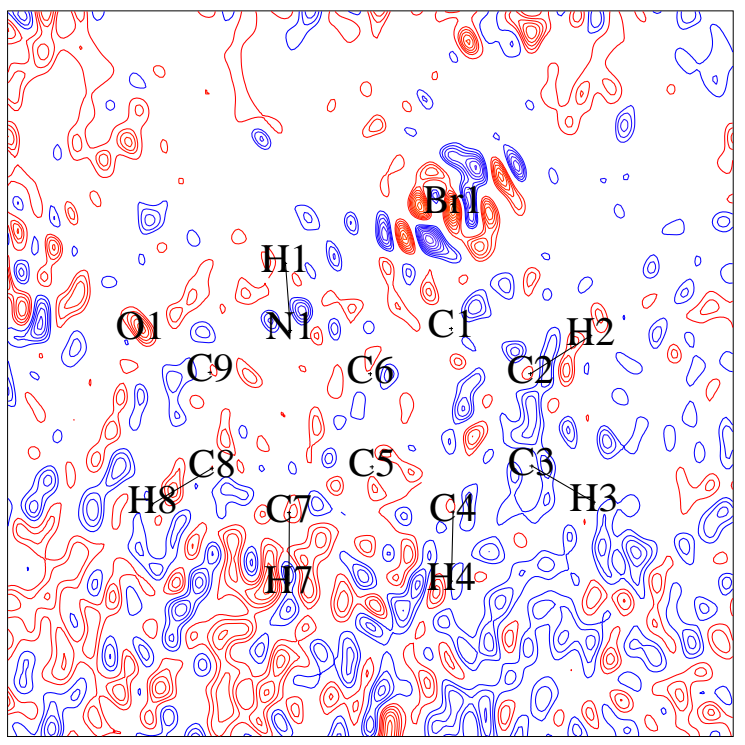

(a) XWR, harmonic, $|F| \geq 2 \sigma(|F|)$

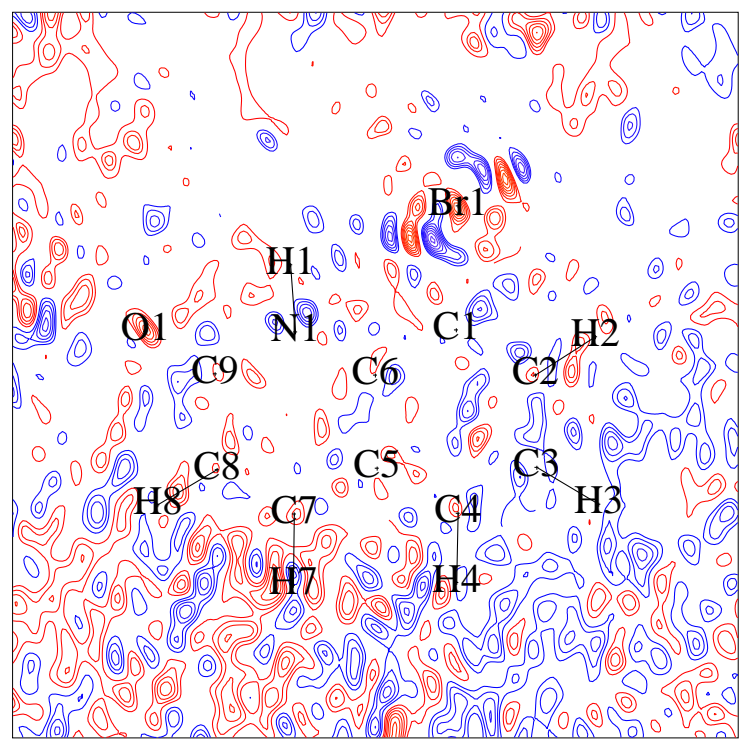

(c) XWR, anharmonic $\mathrm{n}=4,|F| \geq 2 \sigma(|F|)$

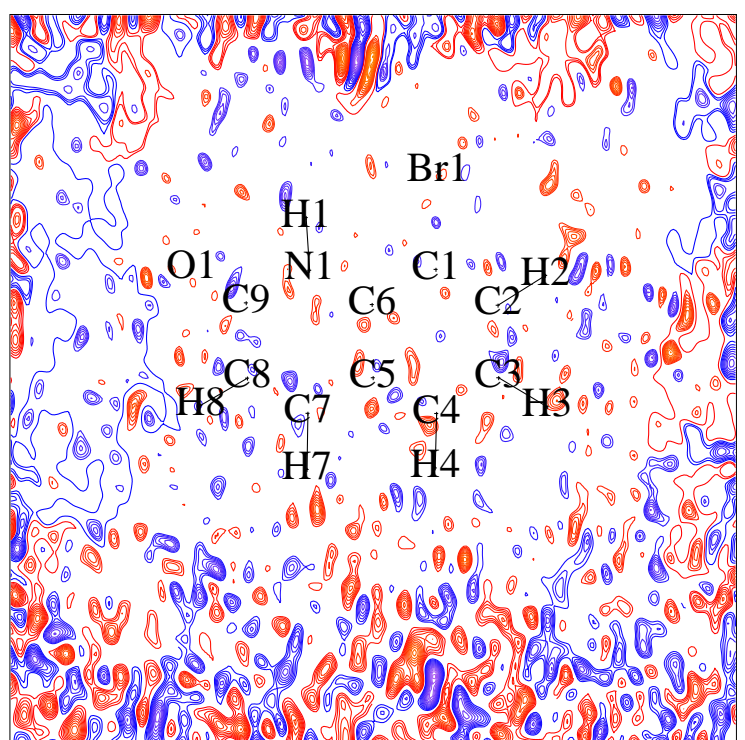

(b) XWR, harmonic, all reflections

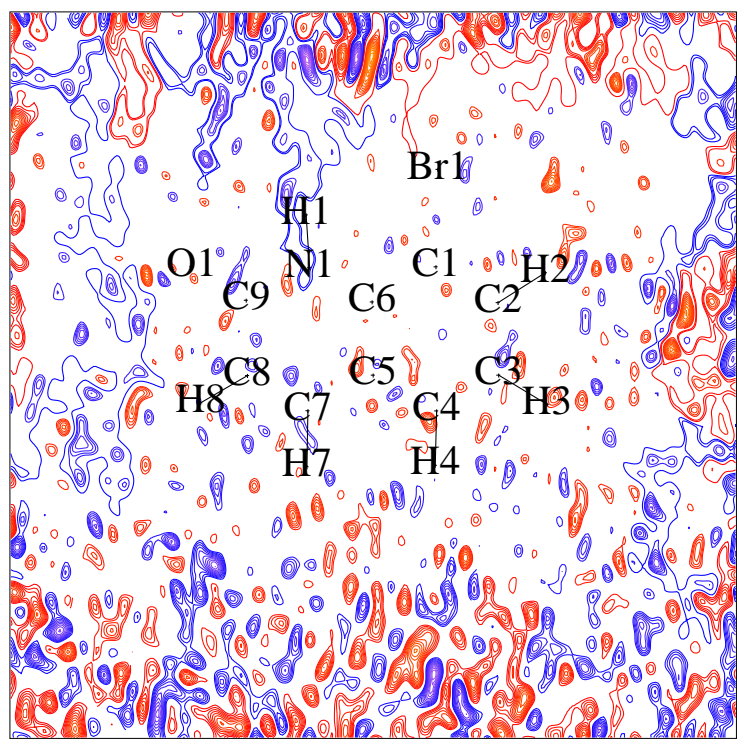

(d) XWR, anharmonic $\mathrm{n}=4$, all reflections

Figure 28: Residual density maps for PT-8, XWR, left: $|F| \geq 2 \sigma(|F|)$ and right: all reflections included. Contour level: 0.05 e/ $\AA^{3}$. Colours: blue - positive, red - negative. 


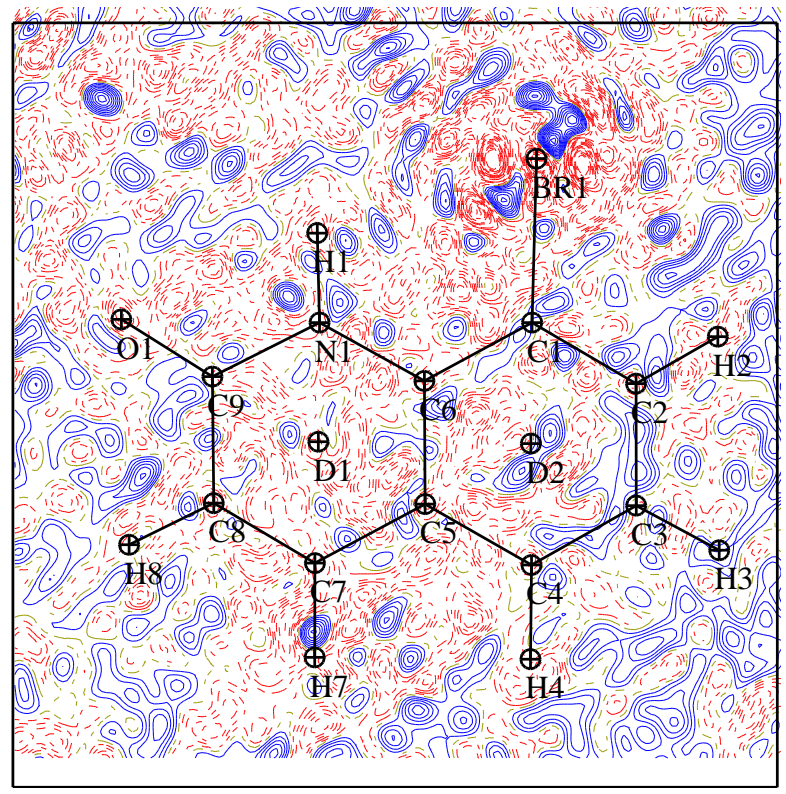

(a) MM, harmonic, $|F| \geq 2 \sigma(|F|)$

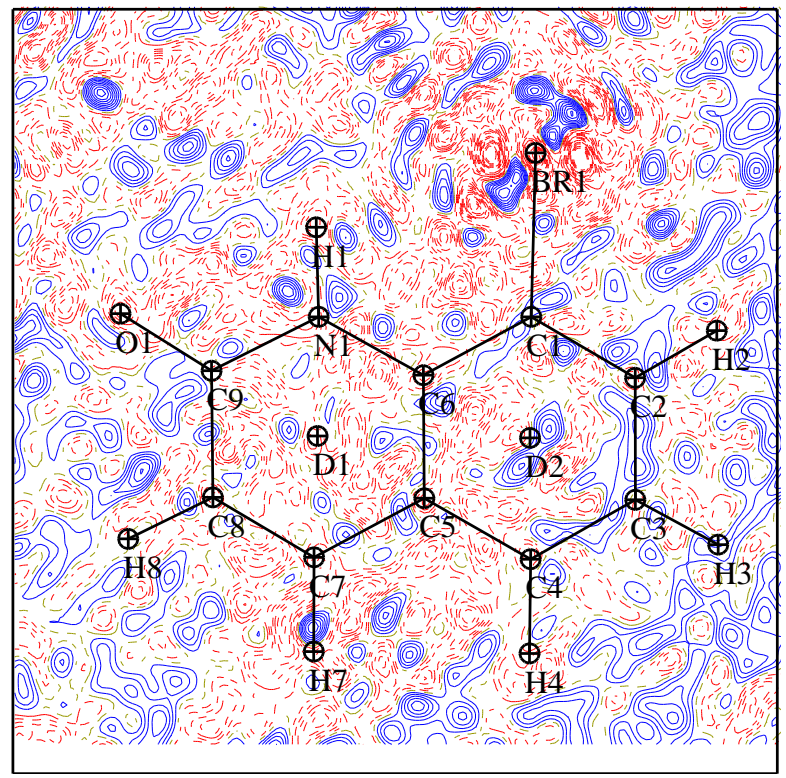

(c) MM, anharmonic $\mathrm{n}=3,|F| \geq 2 \sigma(|F|)$

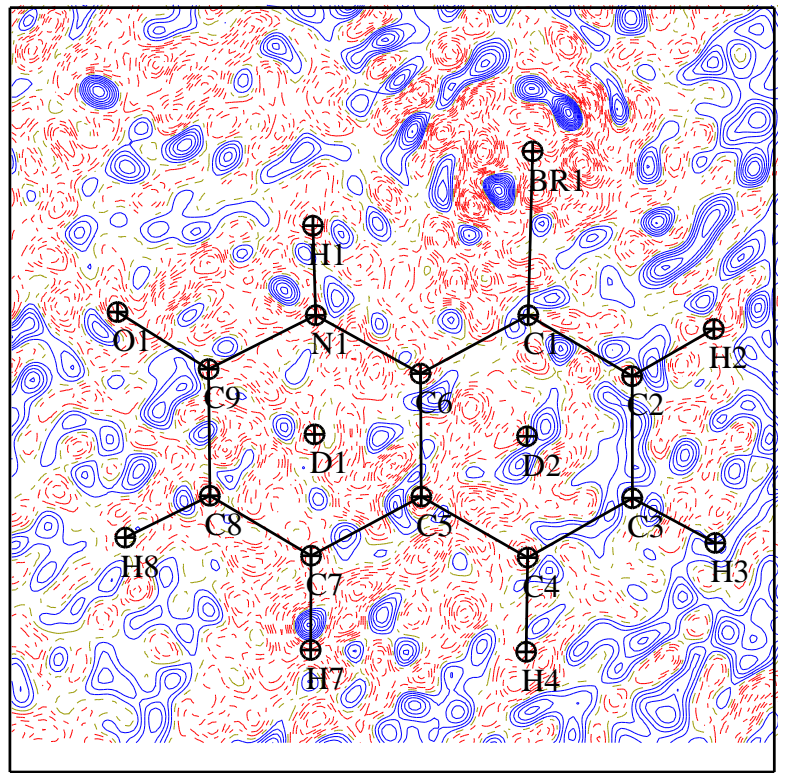

(e) MM, anharmonic $\mathrm{n}=4,|F| \geq 2 \sigma(|F|)$

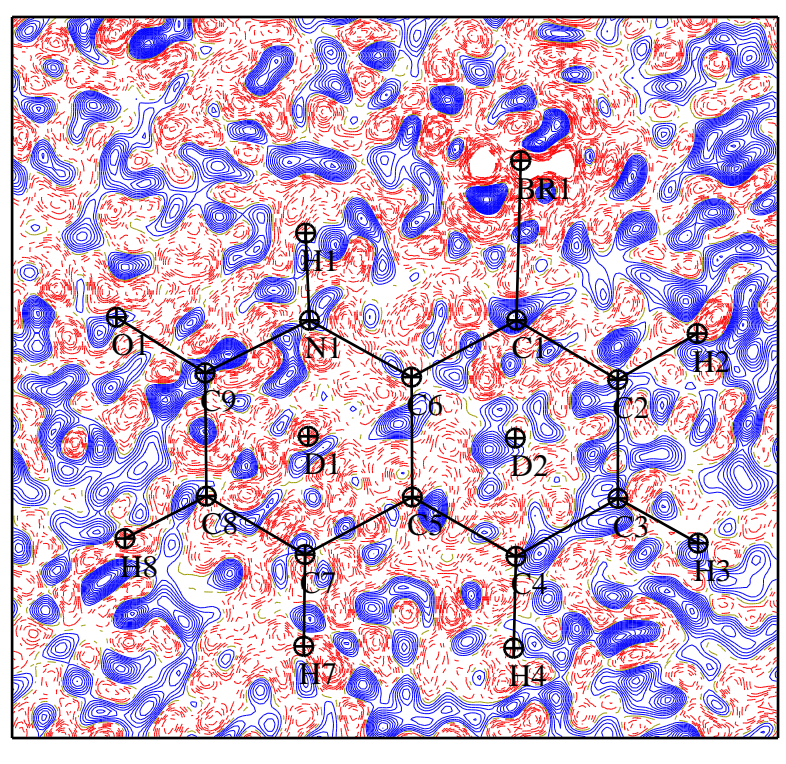

(b) MM, harmonic, all reflections

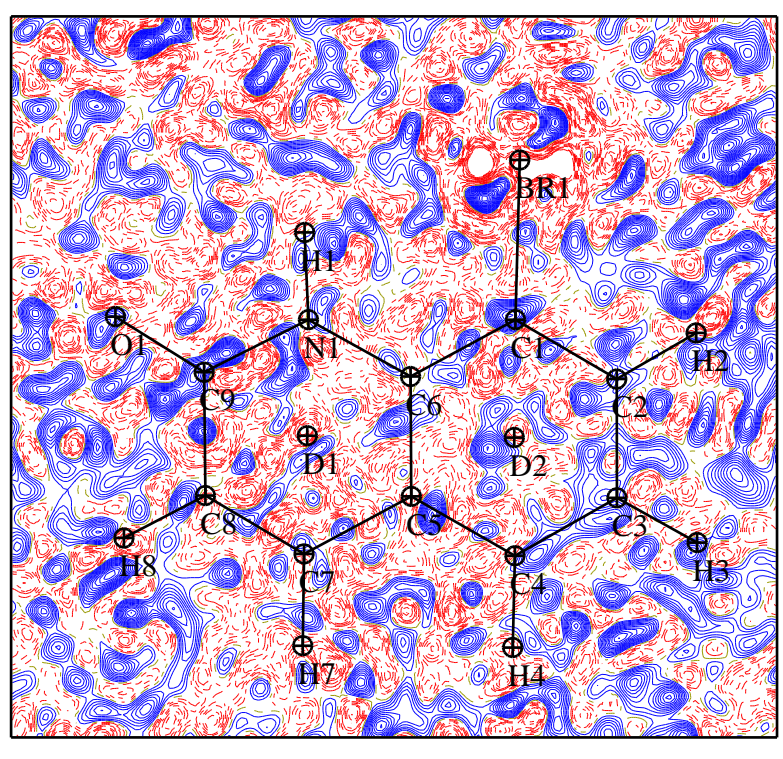

(d) MM, anharmonic $n=3$, all reflections

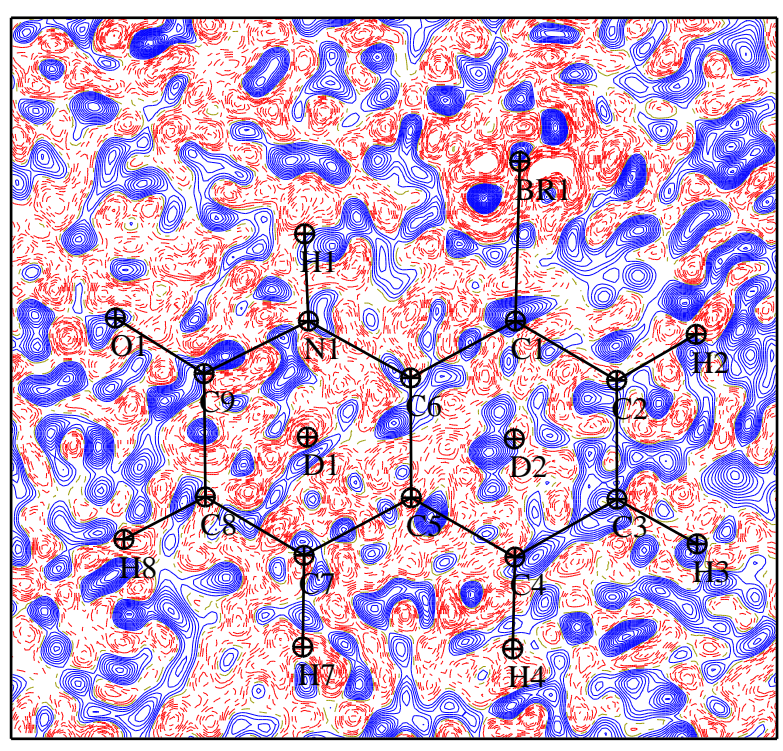

(f) $\mathrm{MM}$, anharmonic $\mathrm{n}=4$, all reflections

Figure 29: Residual density maps for PT-8, MM, restrained X-H distances, left: $|F| \geq 2 \sigma(|F|)$ and right: all reflections included. Contour level: $0.05 \mathrm{e} / \AA^{3}$. Colours: blue - positive, red - negative. 


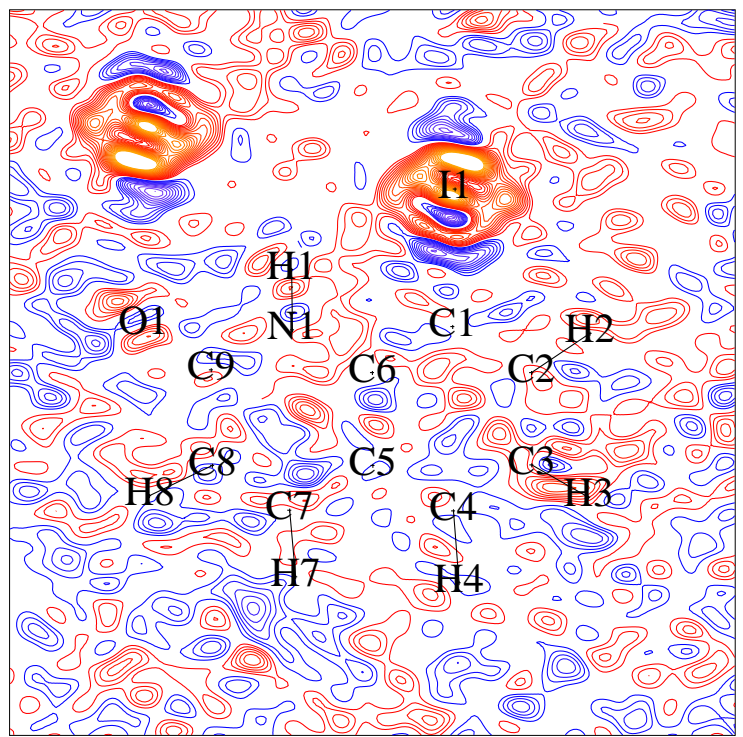

(a) HAR, harmonic, $|F| \geq 2 \sigma(|F|)$

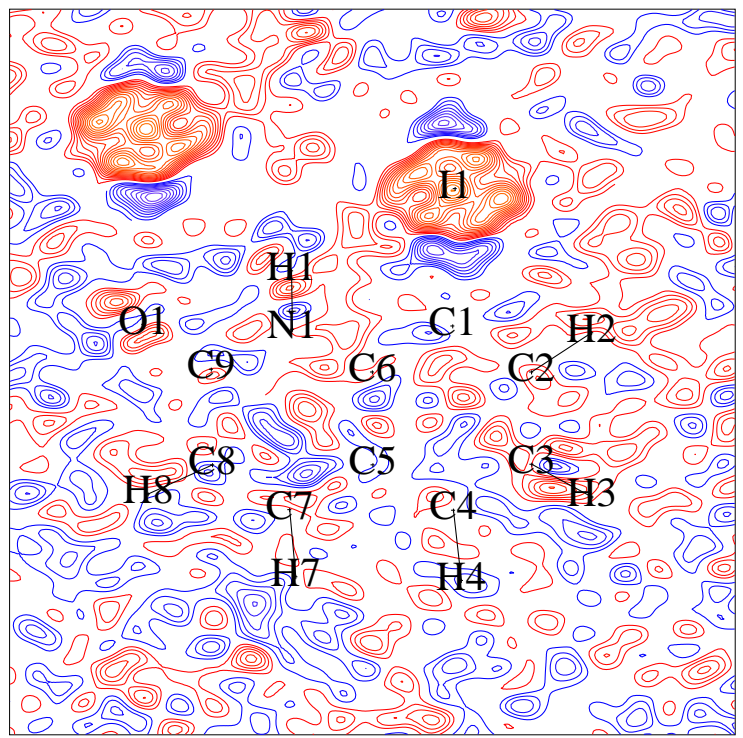

(c) HAR, anharmonic $\mathrm{n}=3,|F| \geq 2 \sigma(|F|)$

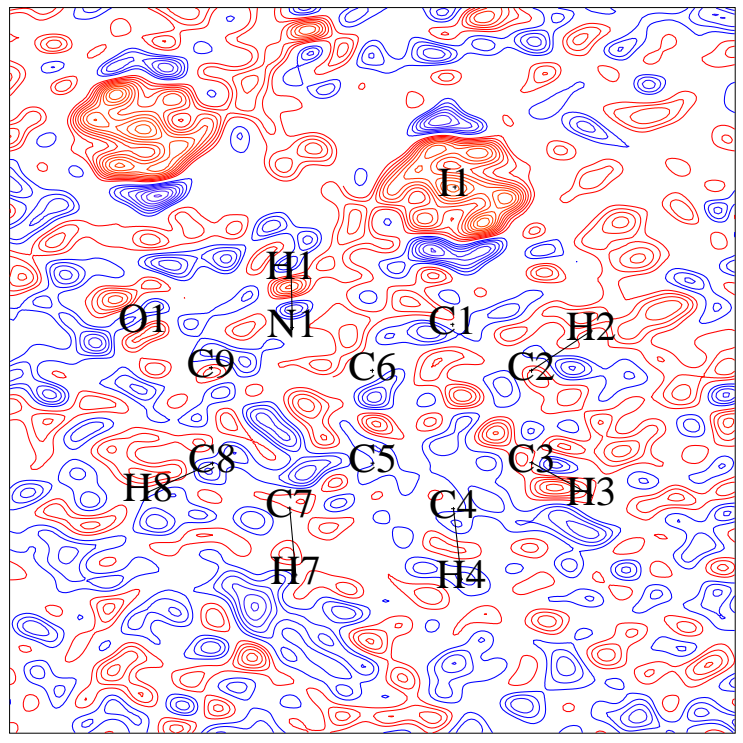

(e) HAR, anharmonic $n=4,|F| \geq 2 \sigma(|F|)$

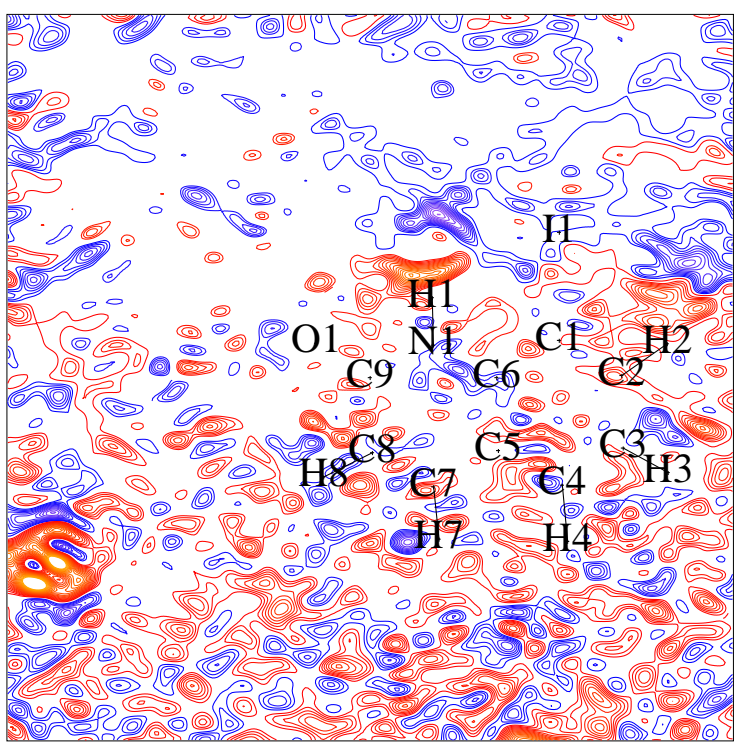

(b) HAR, harmonic, all reflections

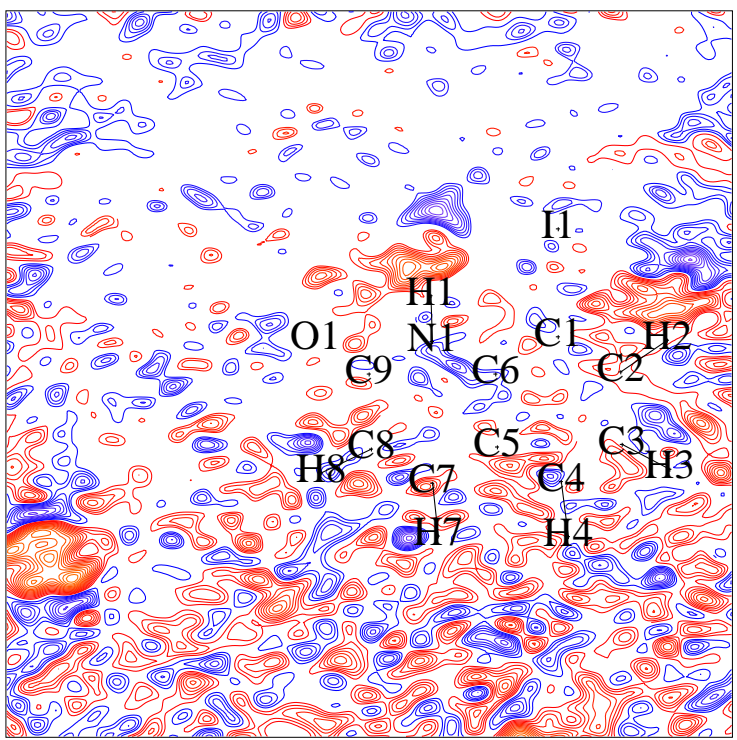

(d) HAR, anharmonic $\mathrm{n}=3$, all reflections

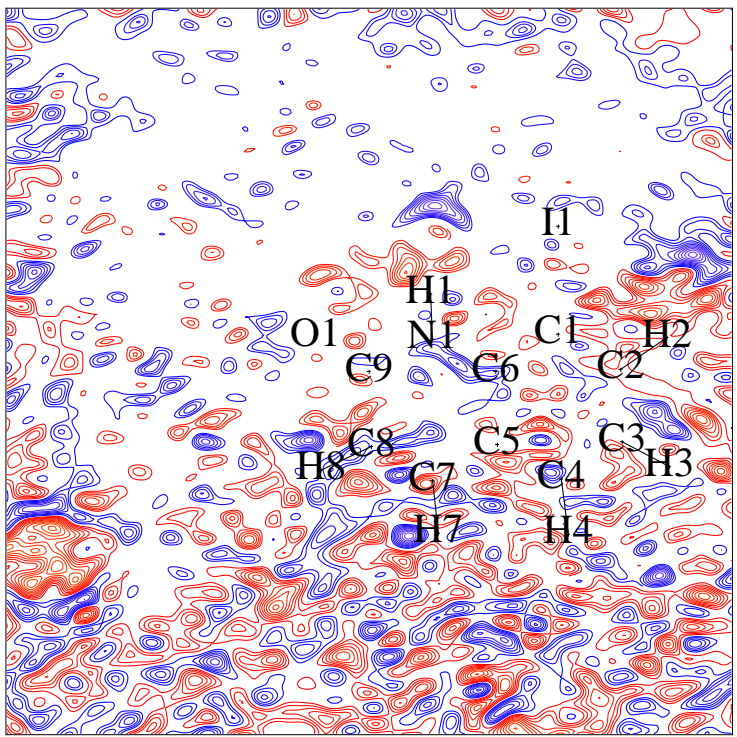

(f) HAR, anharmonic $n=4$, all reflections

Figure 30: Residual density maps for PT-10, HAR, left: $|F| \geq 2 \sigma(|F|)$ and right: all reflections included. Contour level: 0.05 $\mathrm{e} / \AA^{3}$. Colours: blue - positive, red - negative. 


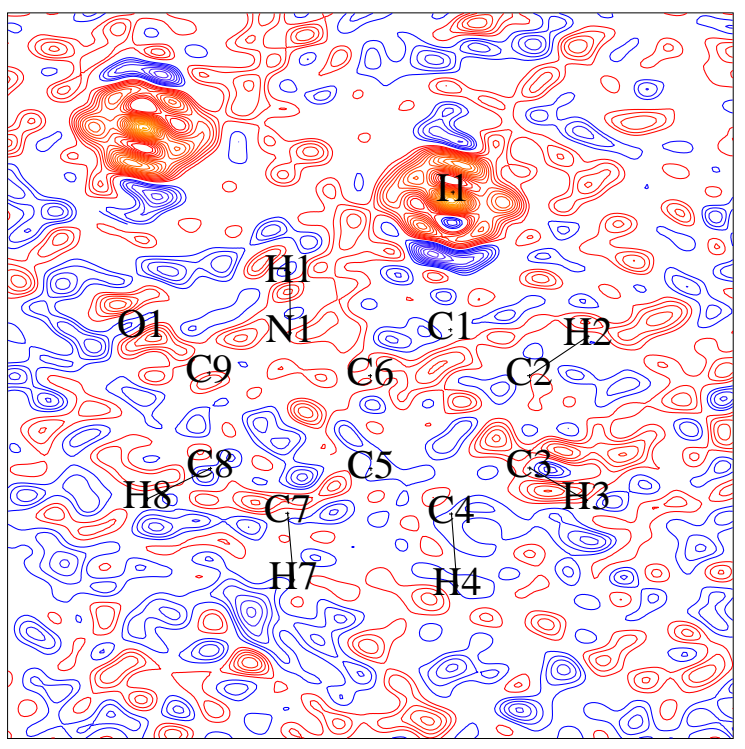

(a) XWR, harmonic, $|F| \geq 2 \sigma(|F|)$

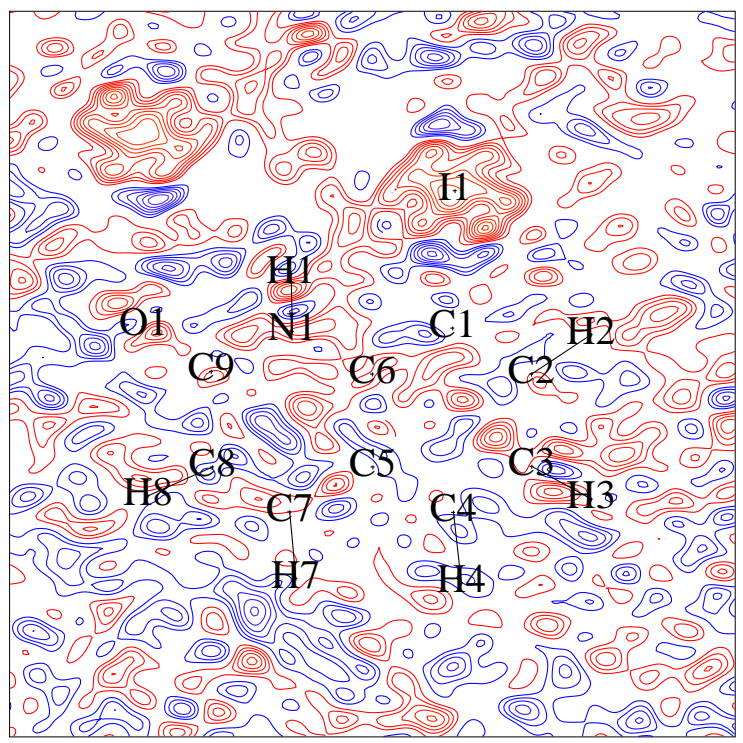

(c) XWR, anharmonic $\mathrm{n}=4,|F| \geq 2 \sigma(|F|)$

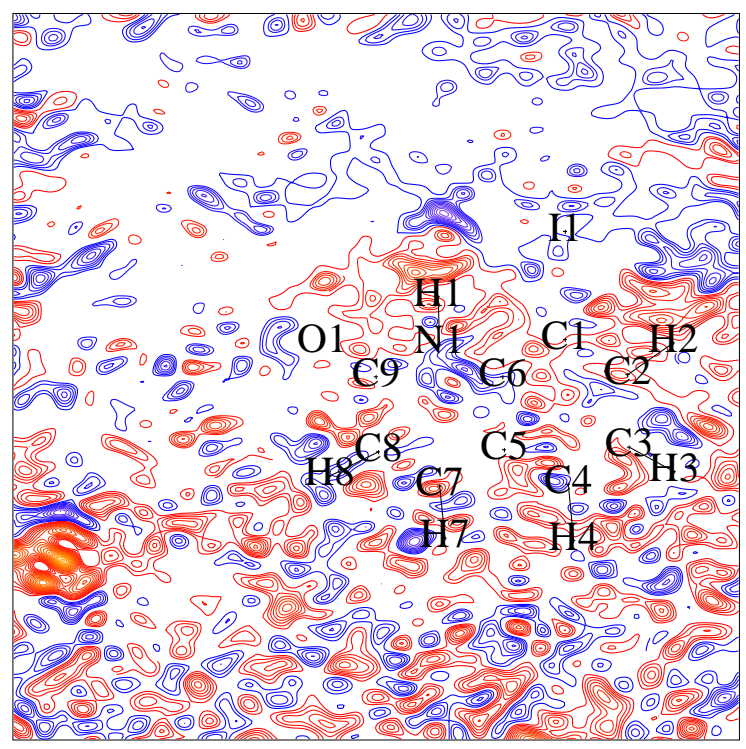

(b) XWR, harmonic, all reflections

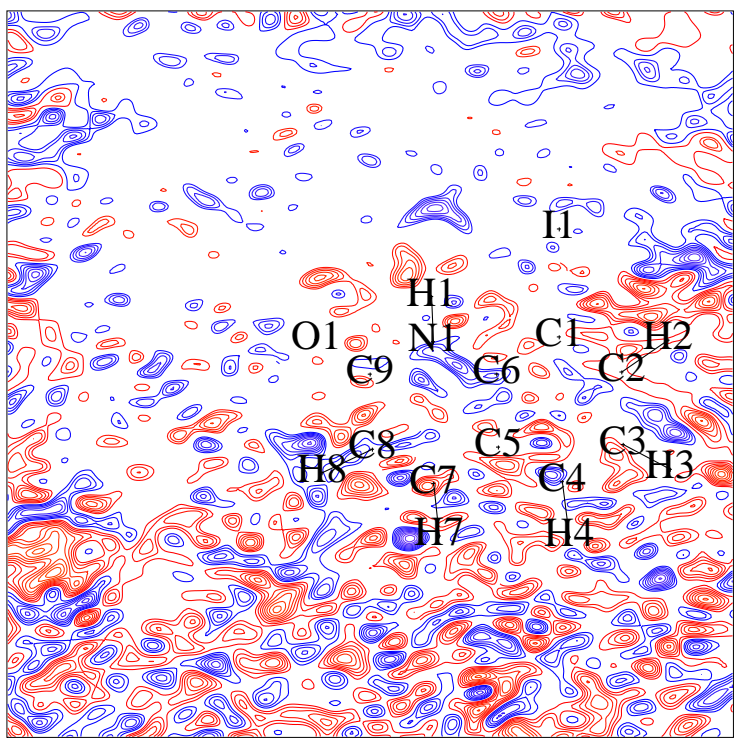

(d) XWR, anharmonic $\mathrm{n}=4$, all reflections

Figure 31: Residual density maps for PT-10, XWR, left: $|F| \geq 2 \sigma(|F|)$ and right: all reflections included. Contour level: 0.05 e/ $\AA^{3}$. Colours: blue - positive, red - negative. 


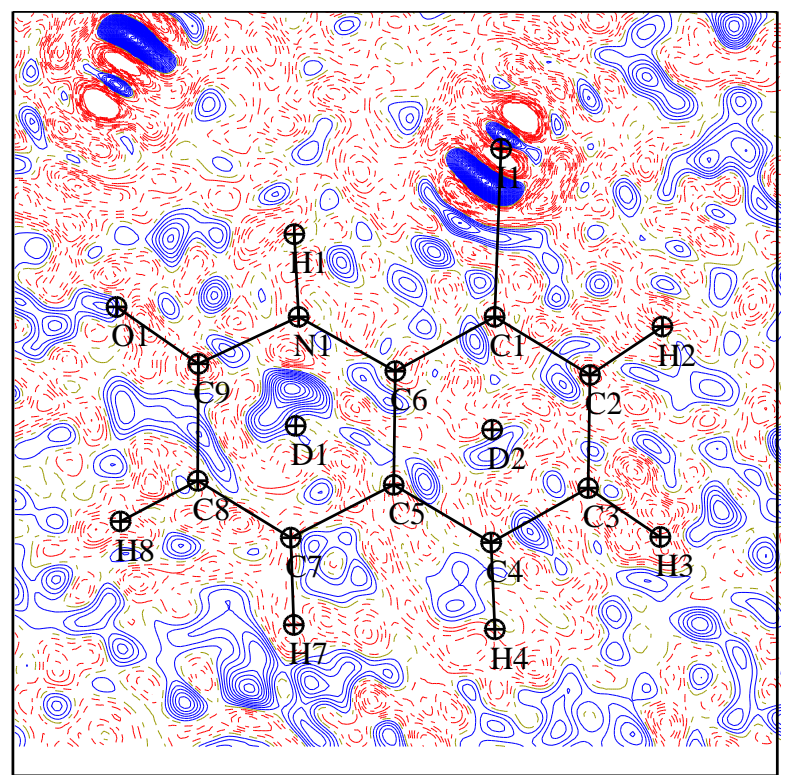

(a) MM, harmonic, $|F| \geq 2 \sigma(|F|)$

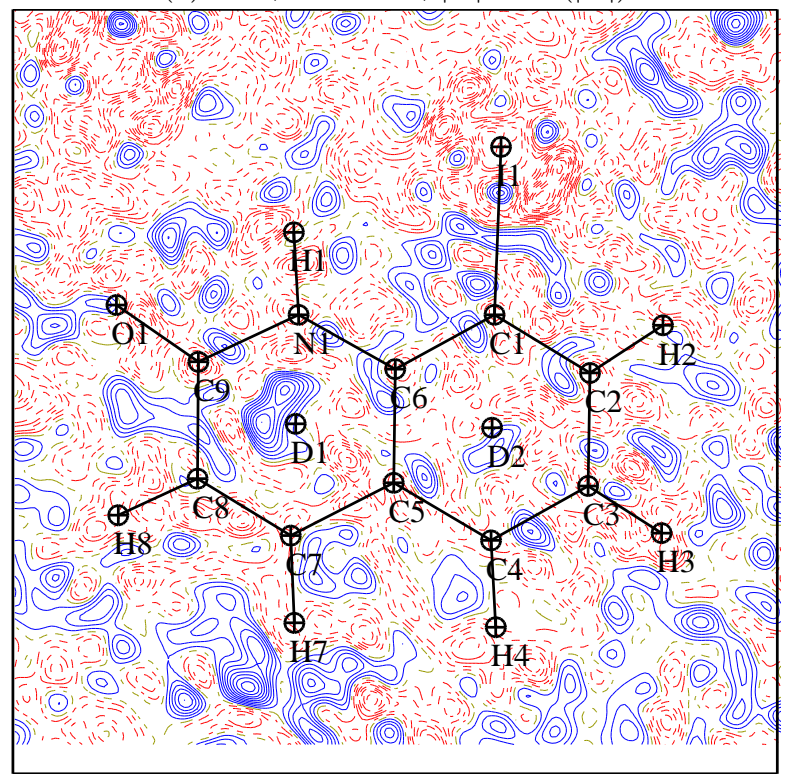

(c) MM, anharmonic $\mathrm{n}=3,|F| \geq 2 \sigma(|F|)$

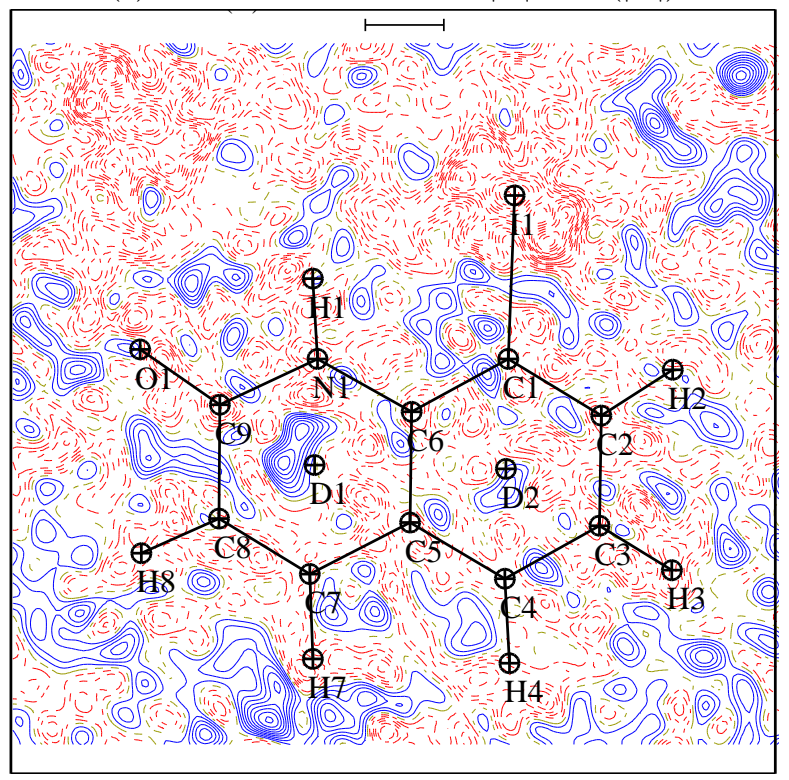

(e) MM, anharmonic $\mathrm{n}=4,|F| \geq 2 \sigma(|F|)$

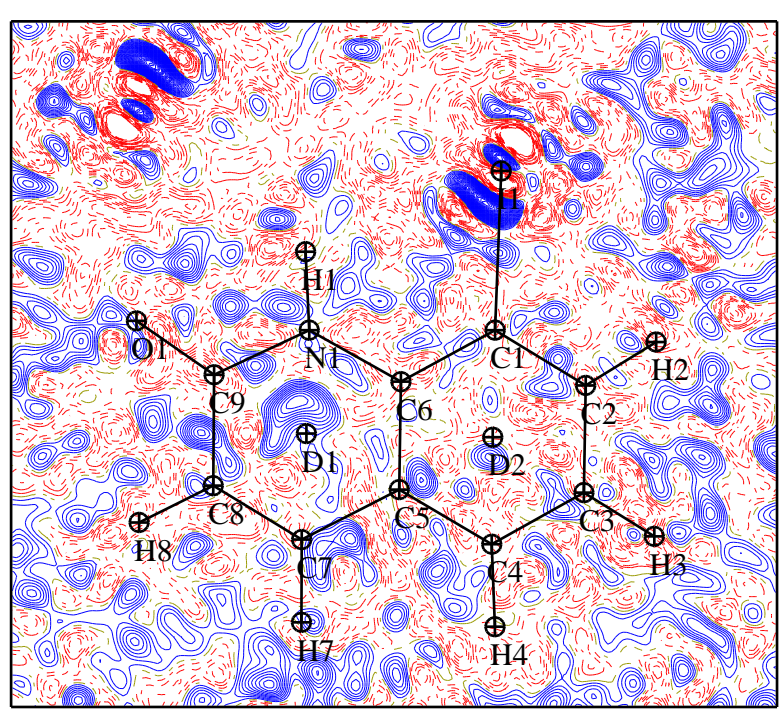

(b) MM, harmonic, all reflections

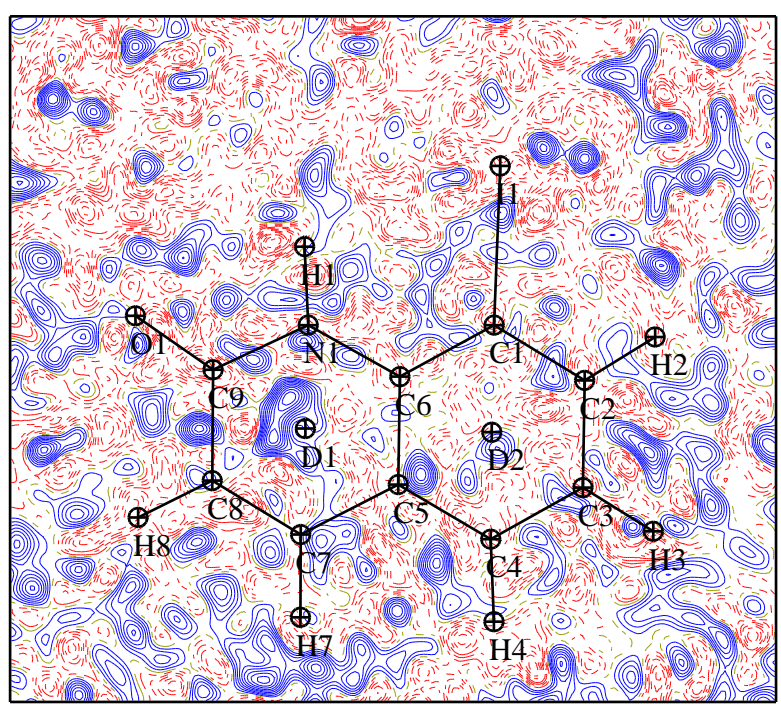

(d) MM, anharmonic $\mathrm{n}=3$, all reflections

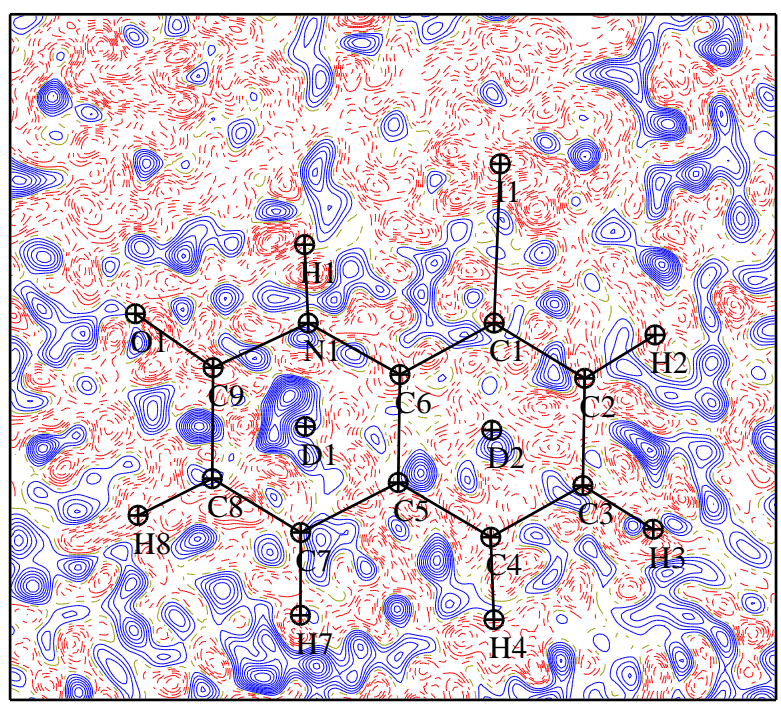

(f) $\mathrm{MM}$, anharmonic $\mathrm{n}=4$, all reflections

Figure 32: Residual density maps for PT-10, MM, restrained X-H distances, left: $|F| \geq 2 \sigma(|F|)$ and right: all reflections included. Contour level: $0.05 \mathrm{e} / \AA^{3}$. Colours: blue - positive, red - negative. 
5 Deformation density maps 


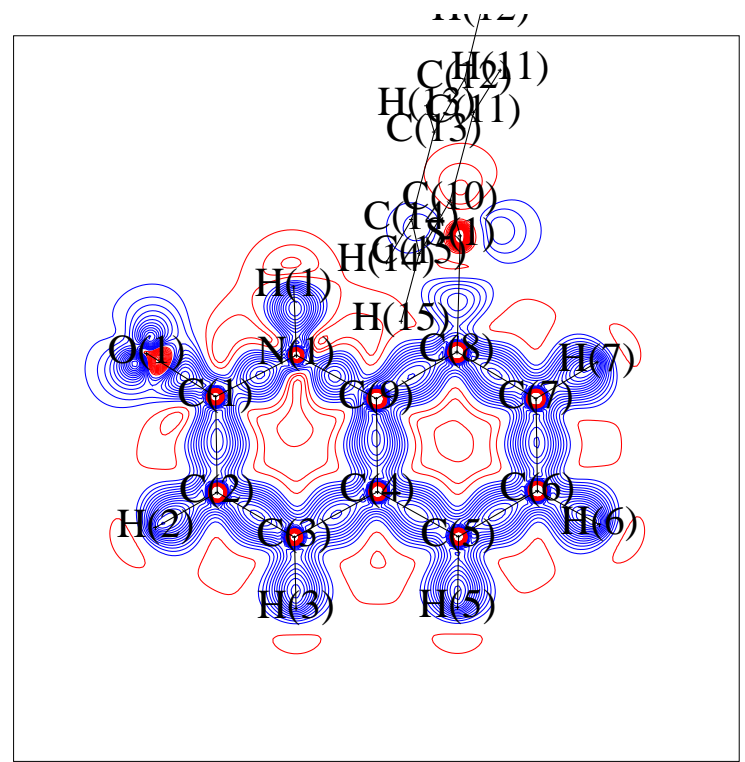

(a) HAR, harmonic, $|F| \geq 2 \sigma(|F|)$

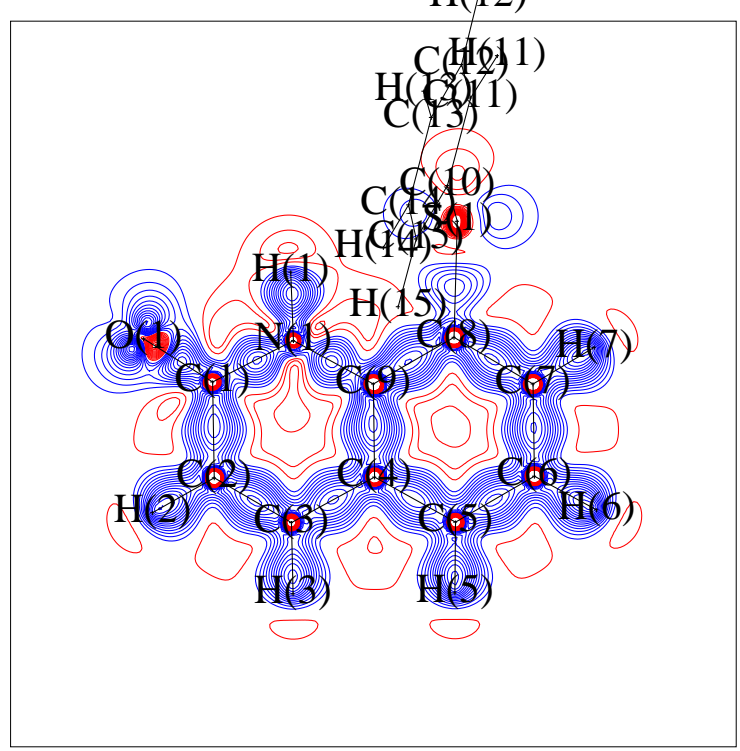

(c) HAR, anharmonic n $=3,|F| \geq 2 \sigma(|F|)$

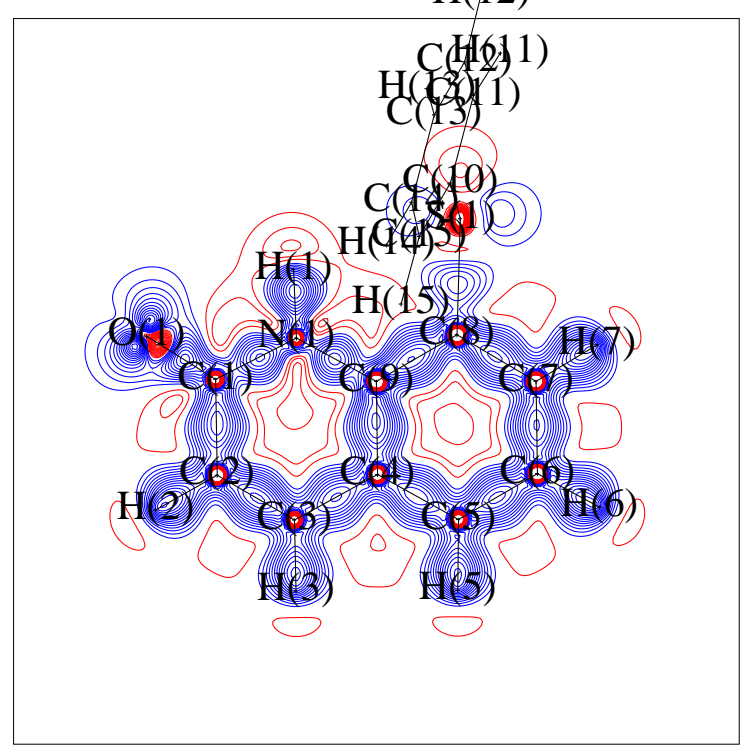

(e) HAR, anharmonic n=4, $|F| \geq 2 \sigma(|F|)$

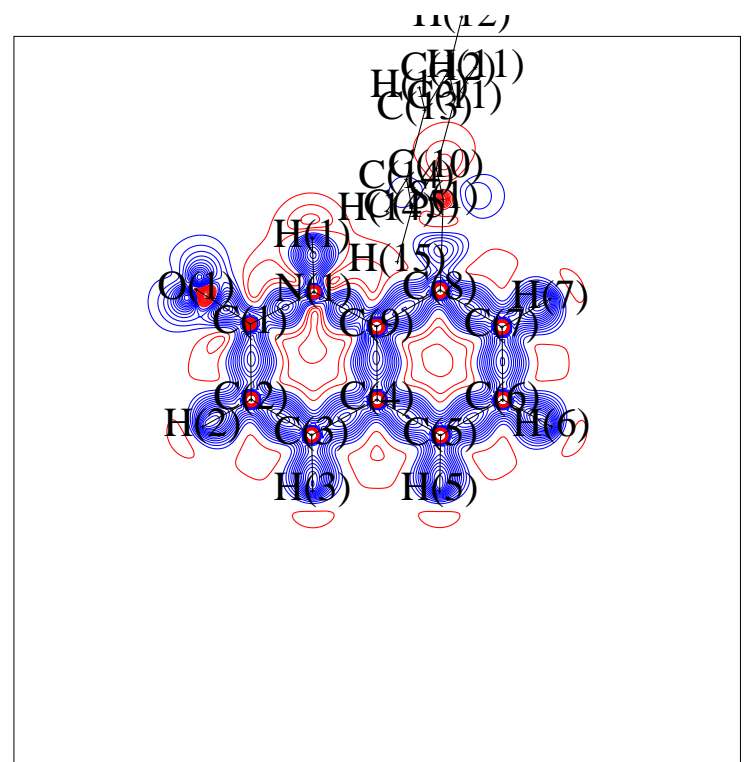

(b) HAR, harmonic, all reflections

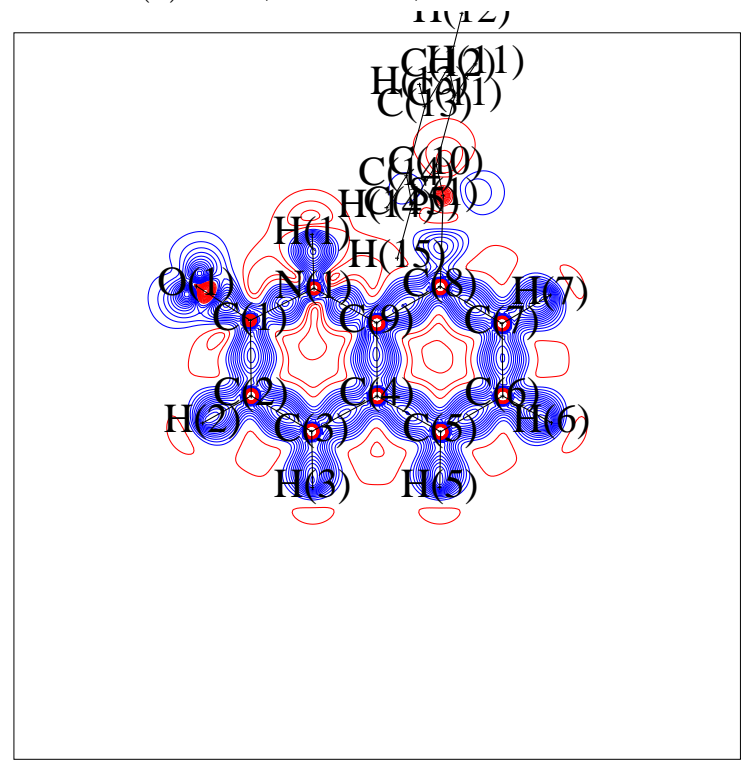

(d) HAR, anharmonic $\mathrm{n}=3$, all reflections

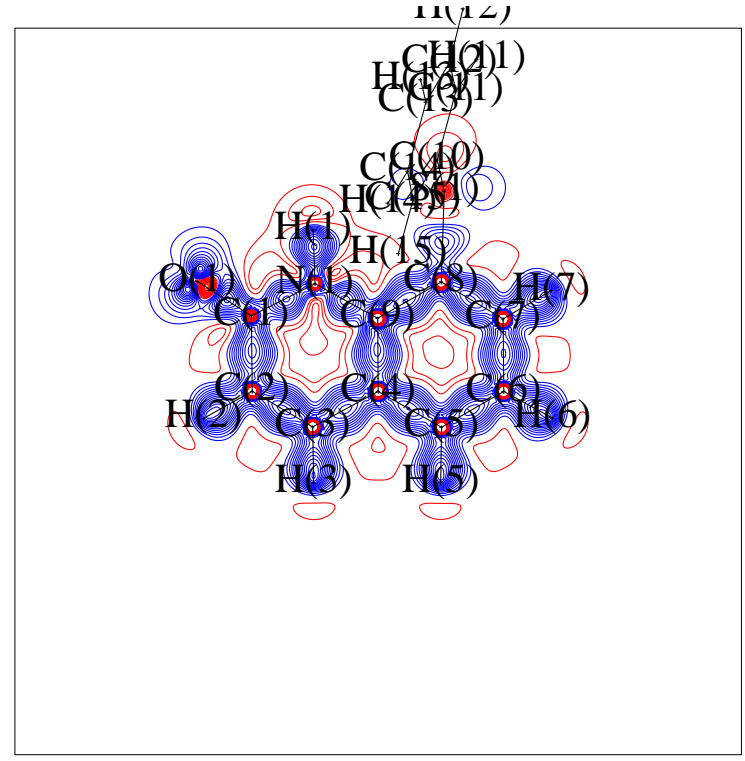

(f) HAR, anharmonic $\mathrm{n}=4$, all reflections

Figure 33: Deformation density maps for PT-11 in the plane of quinoline, HAR, left: $|F| \geq 2 \sigma(|F|)$ and right: all reflections included. Contour level: $0.05 \mathrm{e} / \AA^{3}$. Colours: blue - positive, red - negative. 


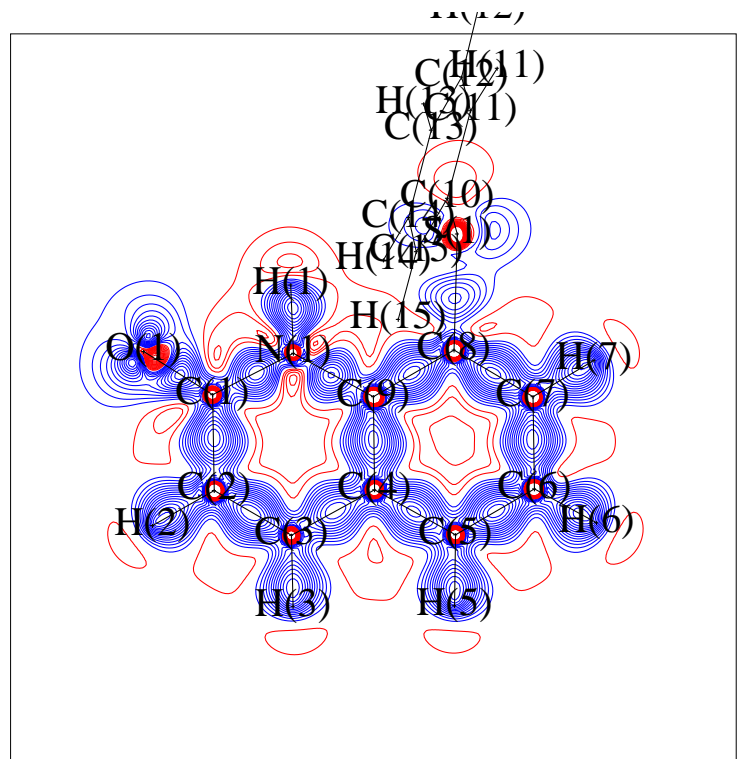

(a) XWR, harmonic, $|F| \geq 2 \sigma(|F|)$

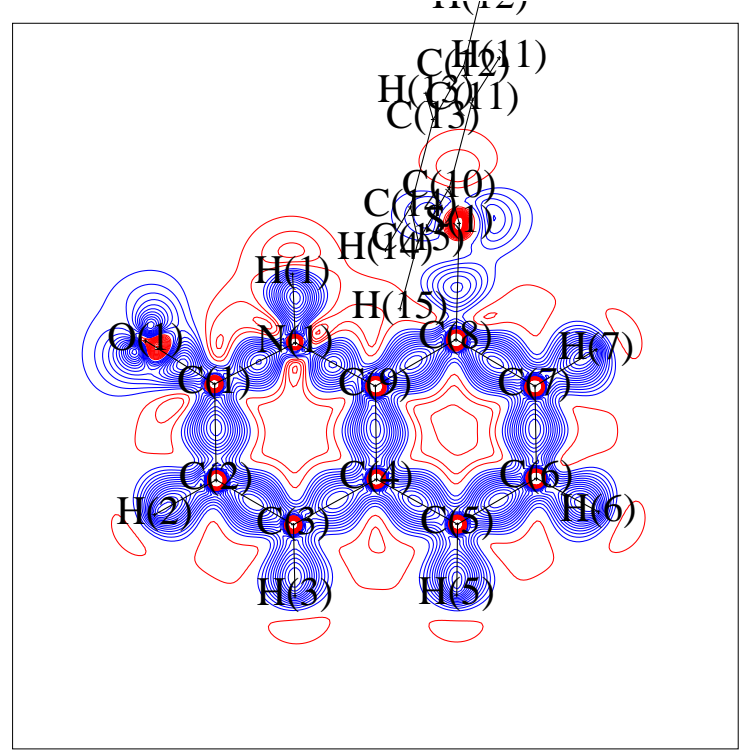

(c) XWR, anharmonic $\mathrm{n}=4,|F| \geq 2 \sigma(|F|)$

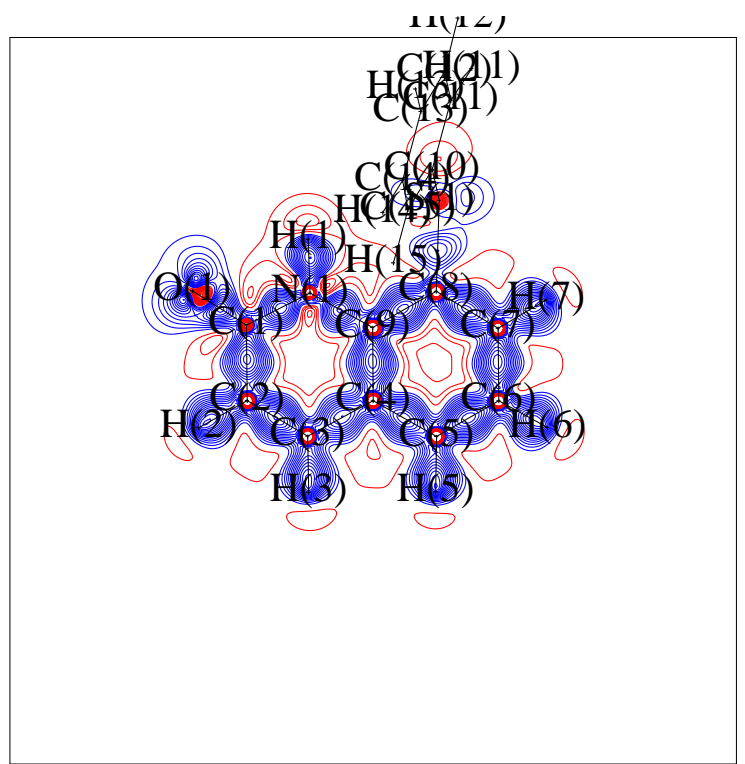

(b) XWR, harmonic, all reflections

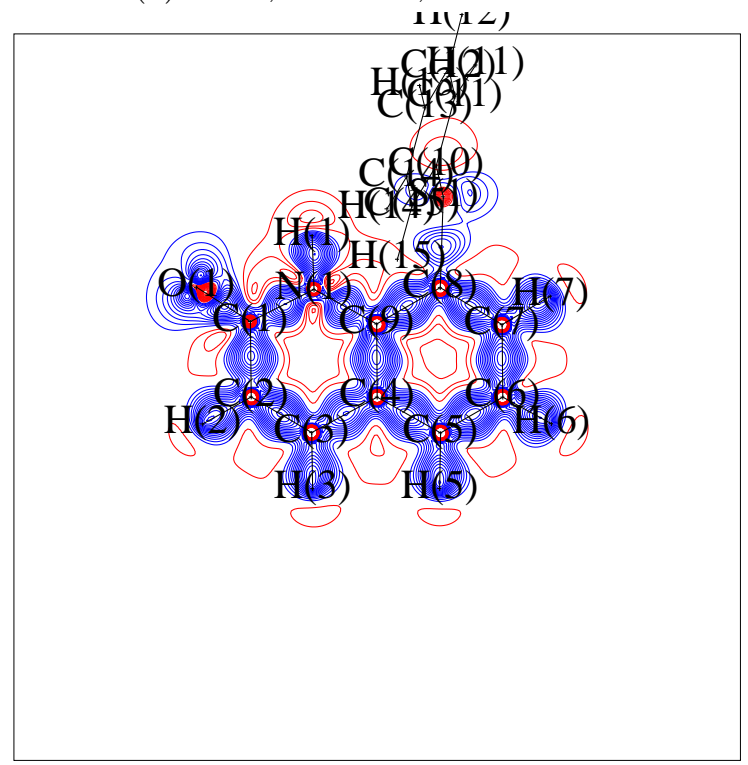

(d) XWR, anharmonic $\mathrm{n}=4$, all reflections

Figure 34: Deformation density maps for PT-11 in the plane of quinoline, XWR, left: $|F| \geq 2 \sigma(|F|)$ and right: all reflections included. Contour level: $0.05 \mathrm{e} / \AA^{3}$. Colours: blue - positive, red - negative. 


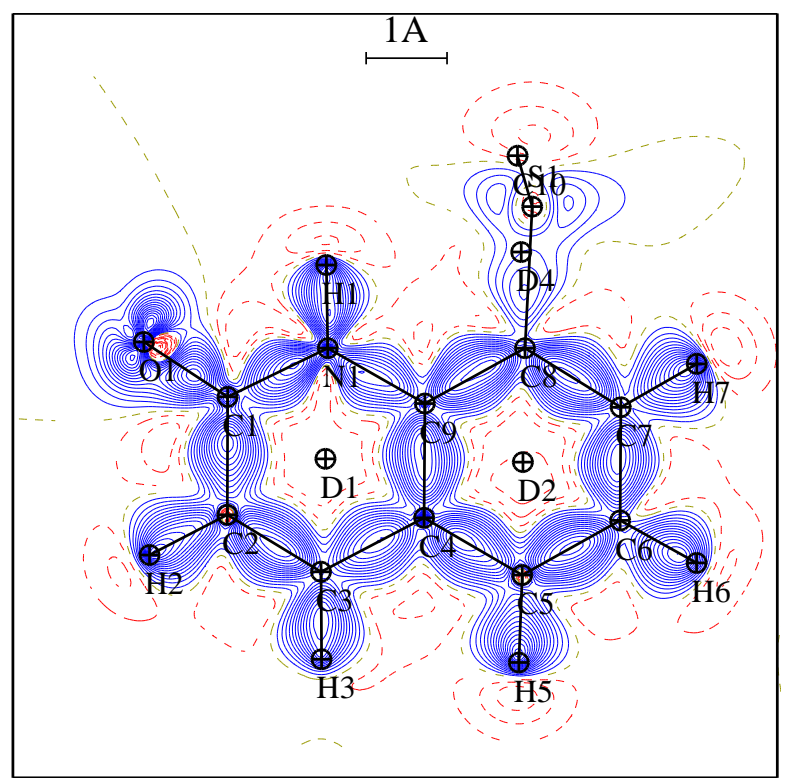

(a) MM, harmonic, $|F| \geq 2 \sigma(|F|)$

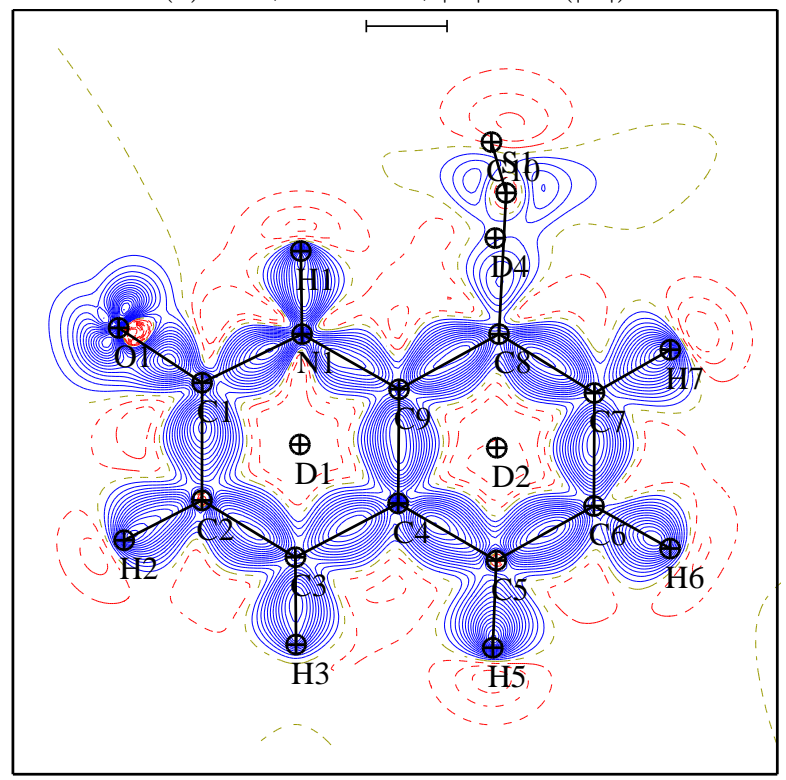

(c) MM, anharmonic $\mathrm{n}=3,|F| \geq 2 \sigma(|F|)$

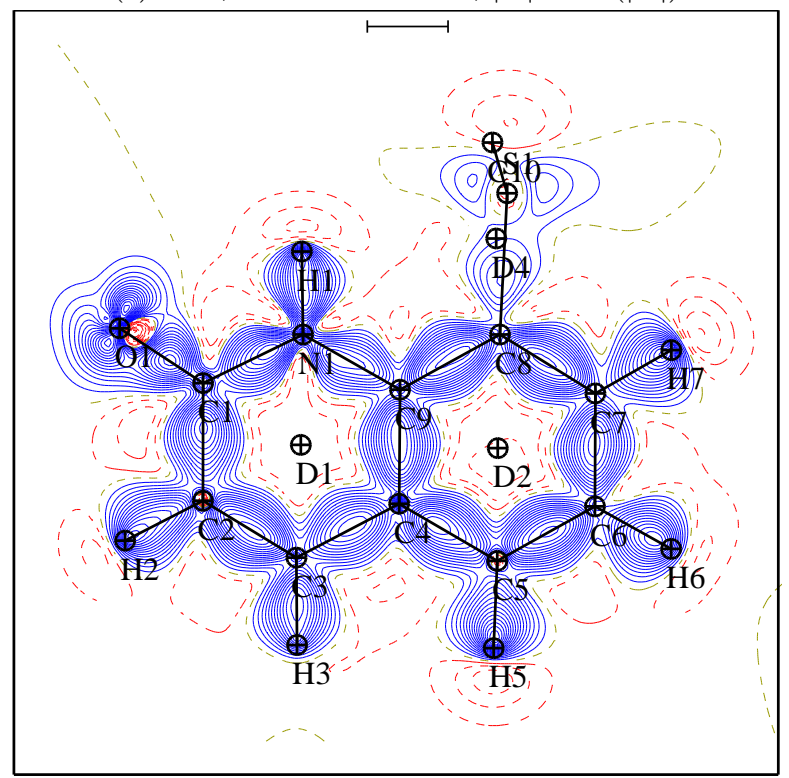

(e) MM, anharmonic $\mathrm{n}=4,|F| \geq 2 \sigma(|F|)$

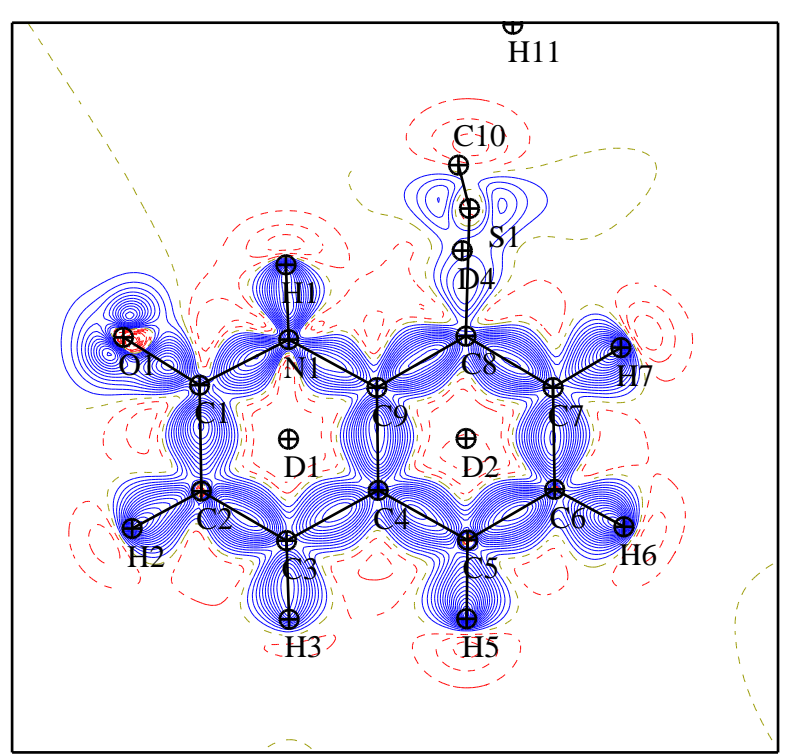

(b) MM, harmonic, all reflections

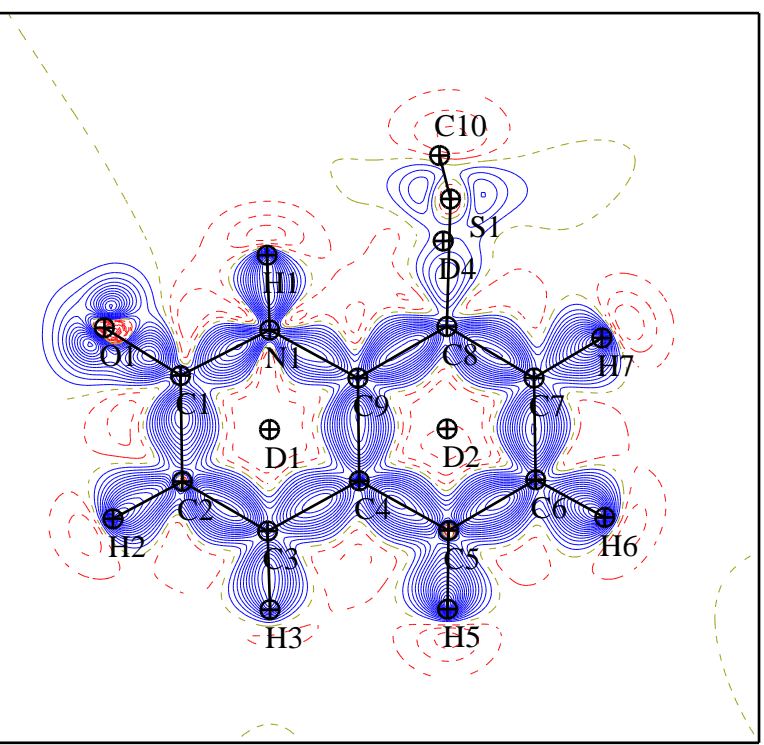

(d) MM, anharmonic $\mathrm{n}=3$, all reflections

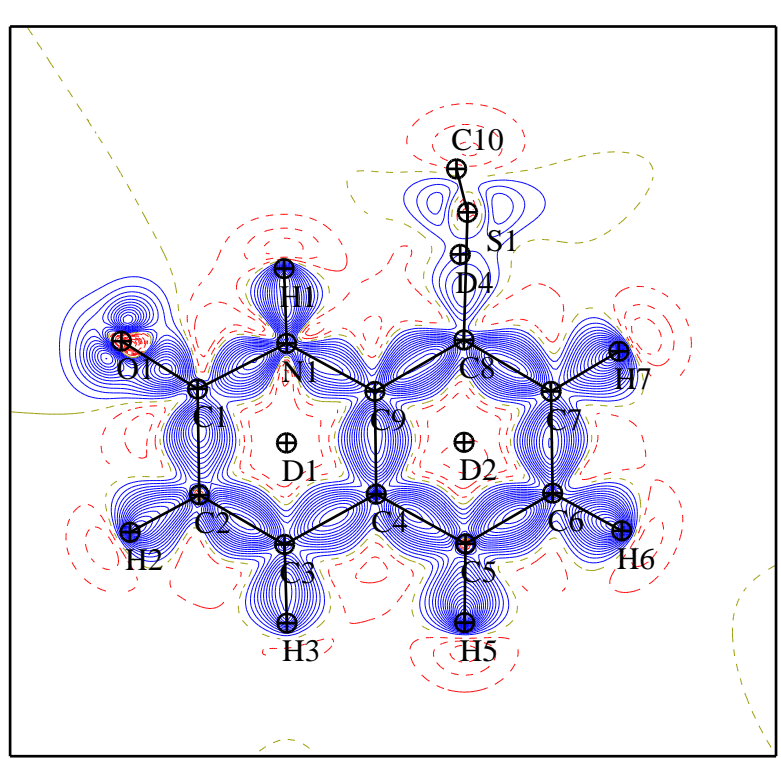

(f) MM, anharmonic $n=4$, all reflections

Figure 35: Deformation density maps for PT-11 in the plane of quinoline, MM, restrained X-H distances, left: $|F| \geq 2 \sigma(|F|)$ and right: all reflections included. Contour level: $0.05 \mathrm{e} / \AA^{3}$. Colours: blue - positive, red - negative. 


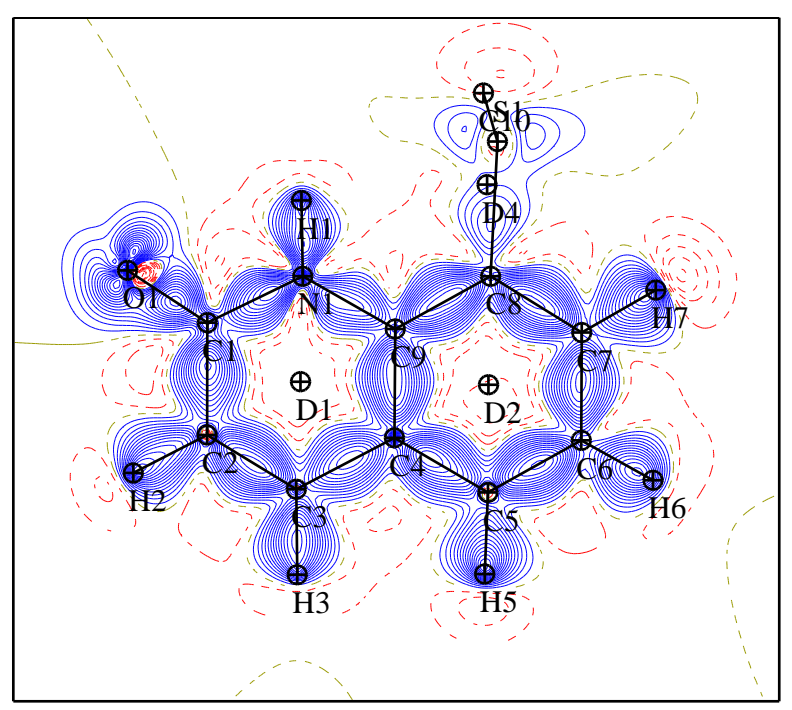

(a) MM, harmonic, $|F| \geq 2 \sigma(|F|)$

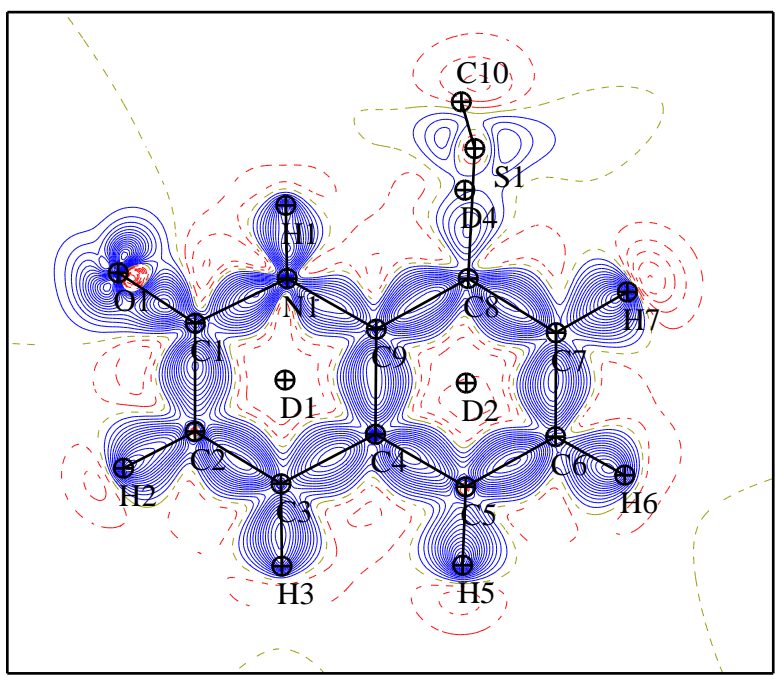

(c) MM, anharmonic $\mathrm{n}=3,|F| \geq 2 \sigma(|F|)$

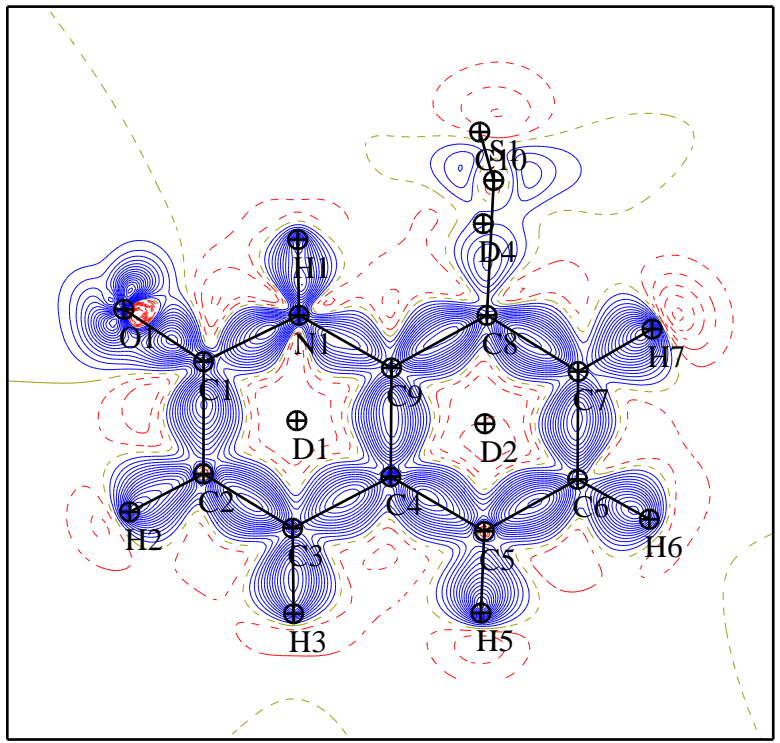

(e) MM, anharmonic $\mathrm{n}=4,|F| \geq 2 \sigma(|F|)$

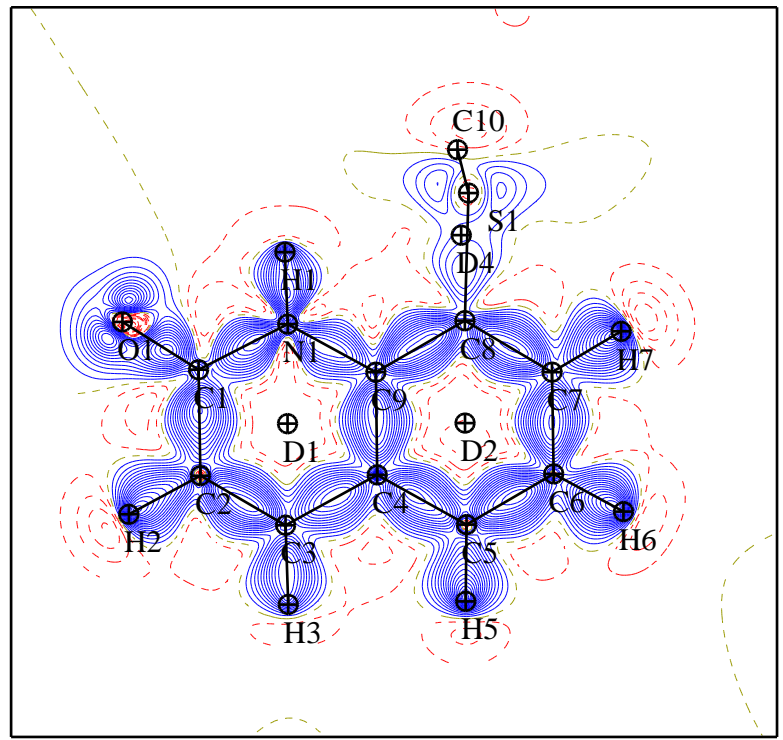

(b) MM, harmonic, all reflections

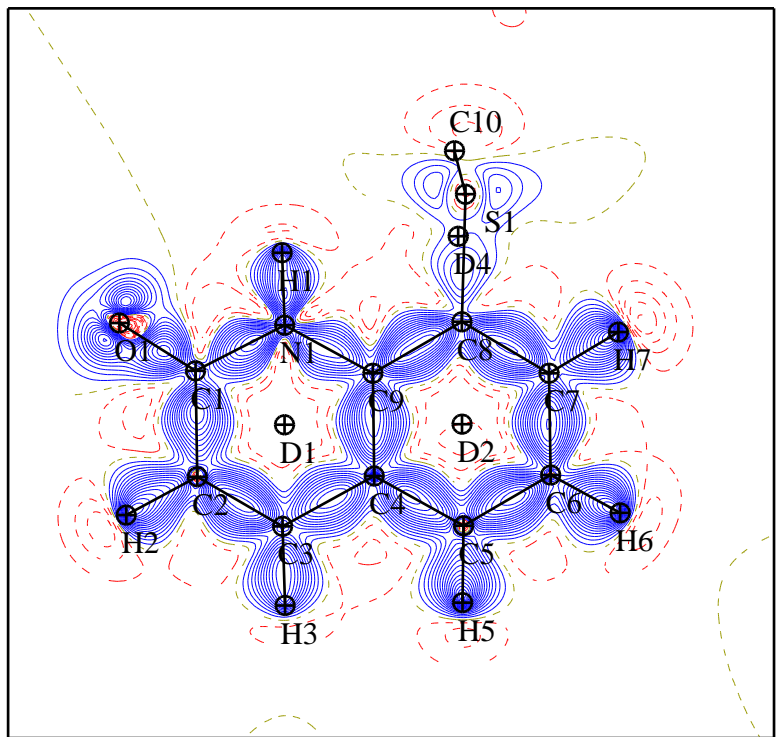

(d) MM, anharmonic $\mathrm{n}=3$, all reflections

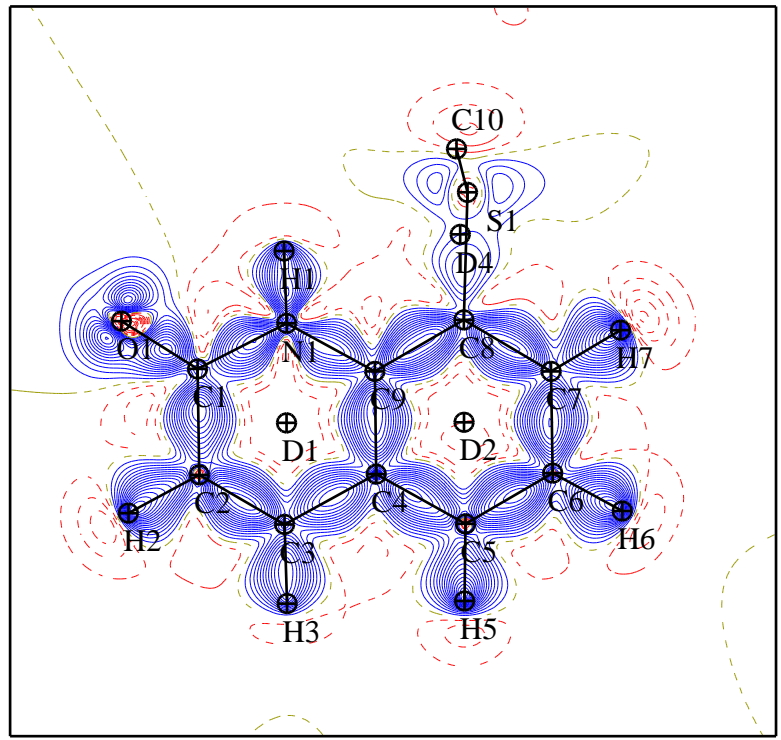

(f) MM, anharmonic $\mathrm{n}=4$, all reflections

Figure 36: Deformation density maps for PT-11 in the plane of quinoline, MM, unrestrained X-H distances, left: $|F| \geq 2 \sigma(|F|)$ and right: all reflections included. Contour level: $0.05 \mathrm{e} / \AA^{3}$. Colours: blue - positive, red - negative. 


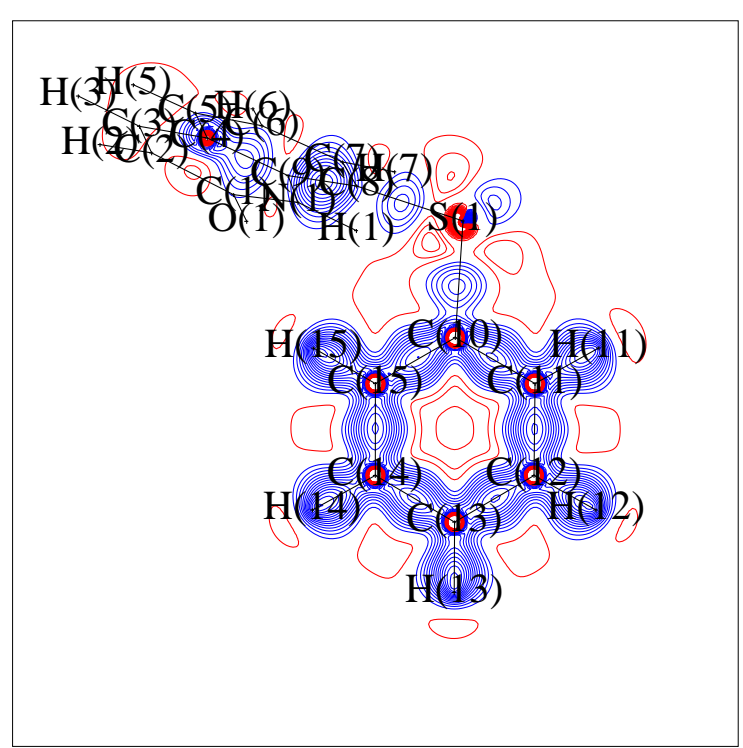

(a) HAR, harmonic, $|F| \geq 2 \sigma(|F|)$

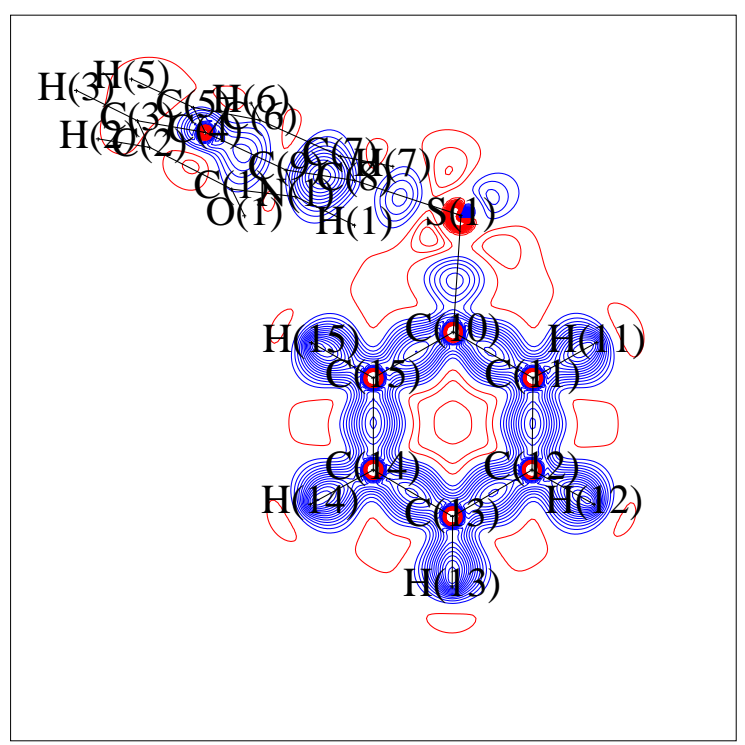

(c) HAR, anharmonic $\mathrm{n}=3,|F| \geq 2 \sigma(|F|)$

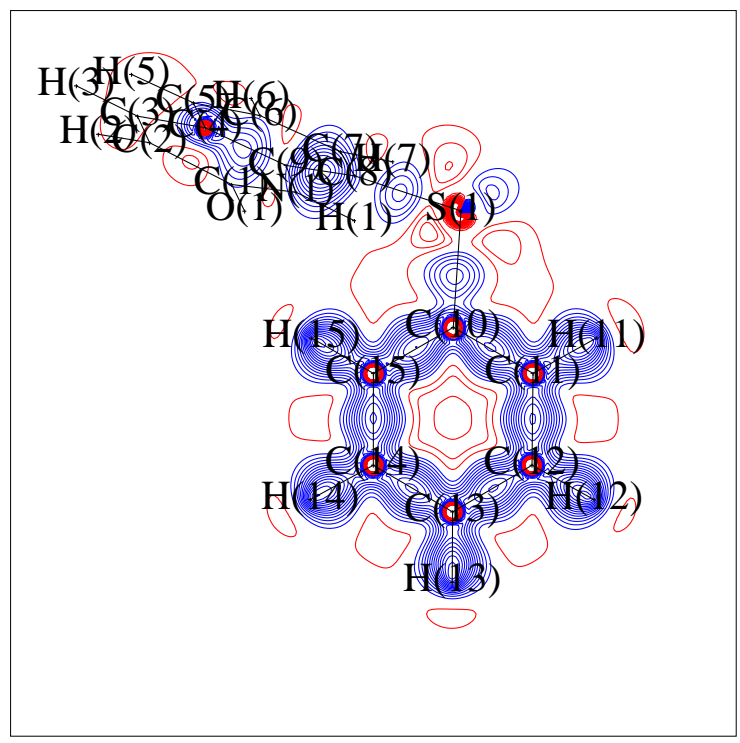

(e) HAR, anharmonic n=4, $|F| \geq 2 \sigma(|F|)$

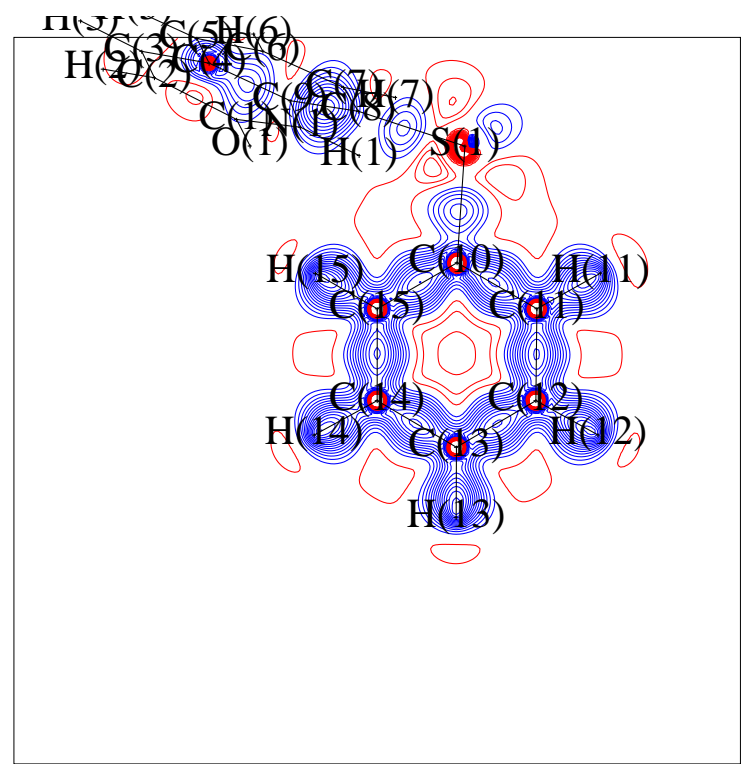

(b) HAR, harmonic, all reflections

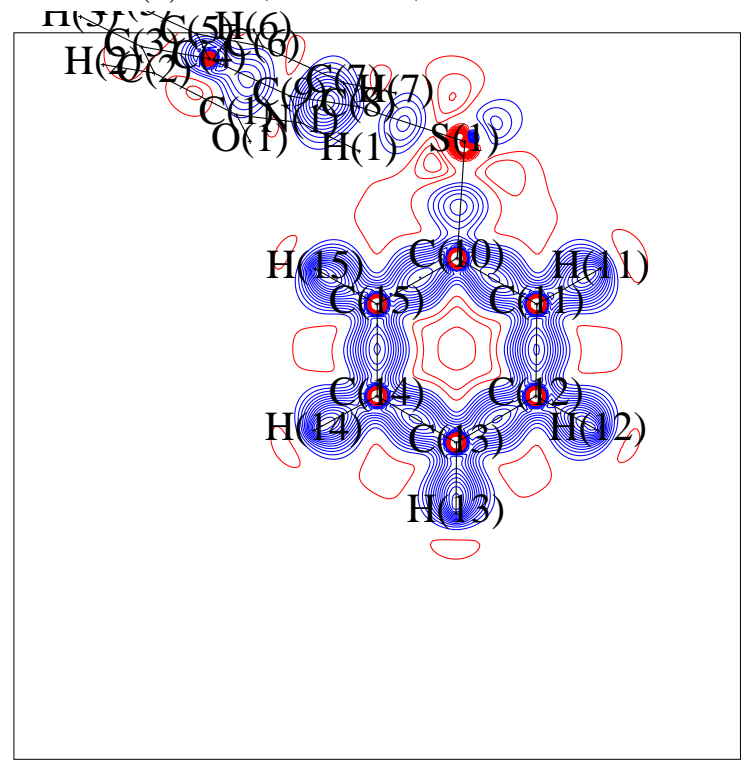

(d) HAR, anharmonic $n=3$, all reflections

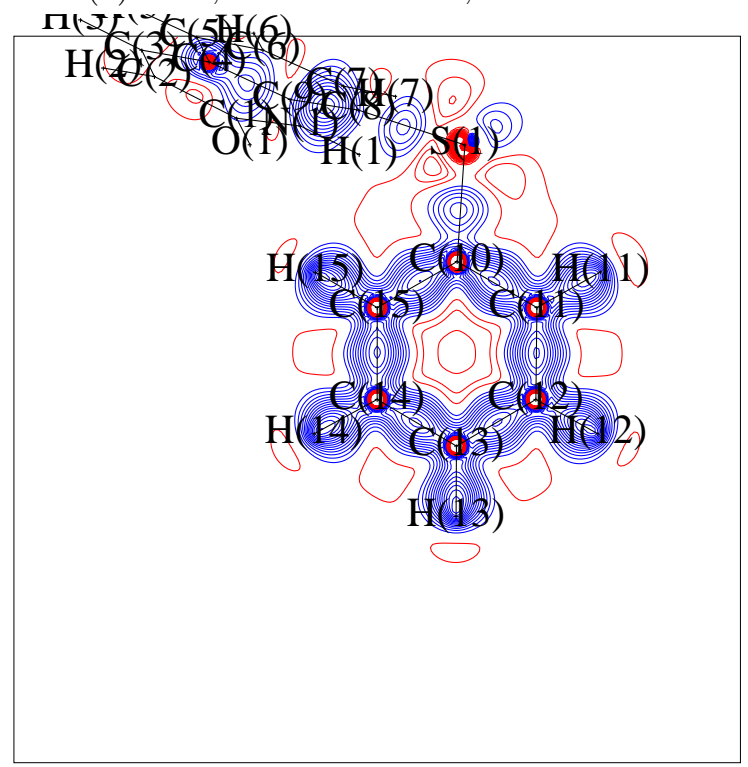

(f) HAR, anharmonic $n=4$, all reflections

Figure 37: Deformation density maps for PT-11 in the plane of phenyl ring, HAR, left: $|F| \geq 2 \sigma(|F|)$ and right: all reflections included. Contour level: $0.05 \mathrm{e} / \AA^{3}$. Colours: blue - positive, red - negative. 


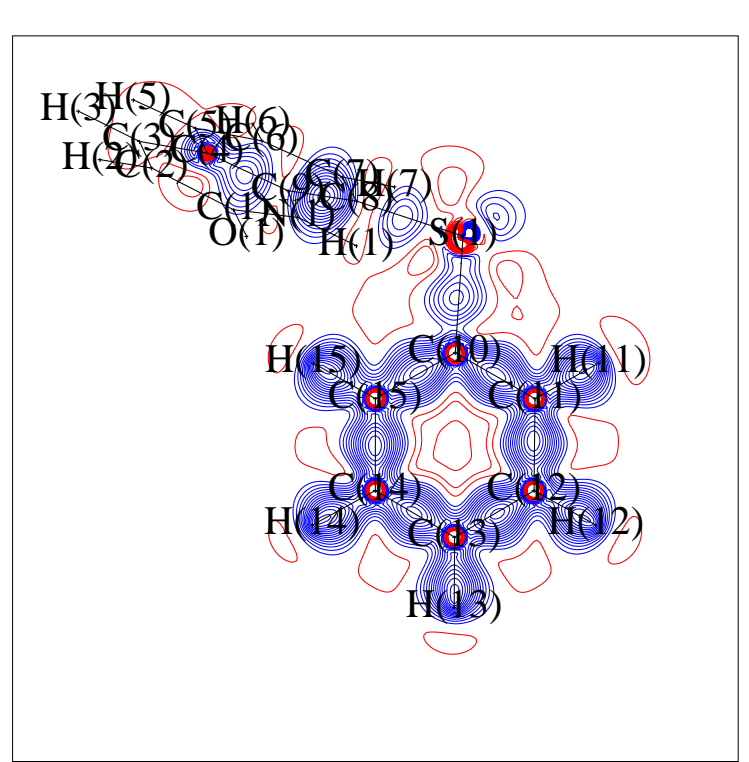

(a) XWR, harmonic, $|F| \geq 2 \sigma(|F|)$

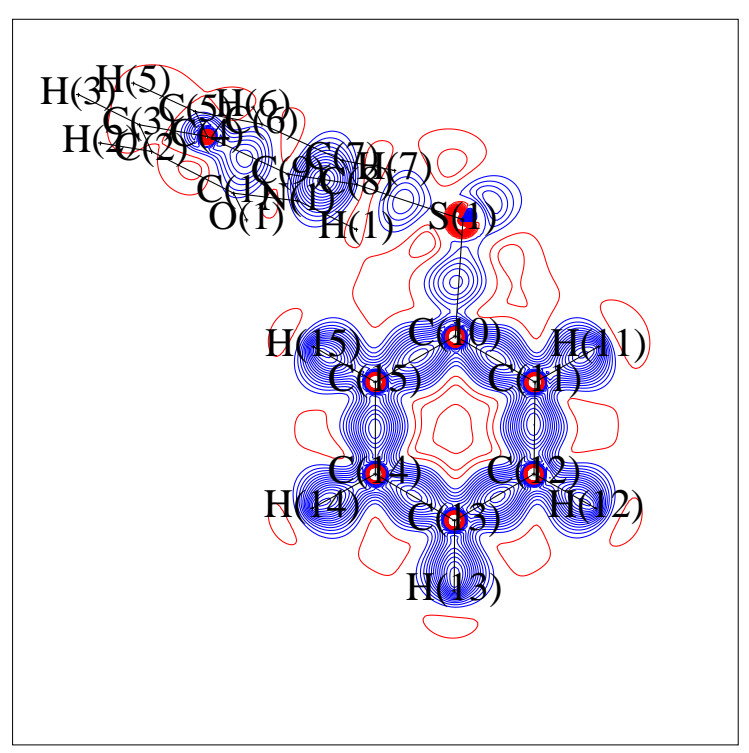

(c) XWR, anharmonic $\mathrm{n}=4,|F| \geq 2 \sigma(|F|)$

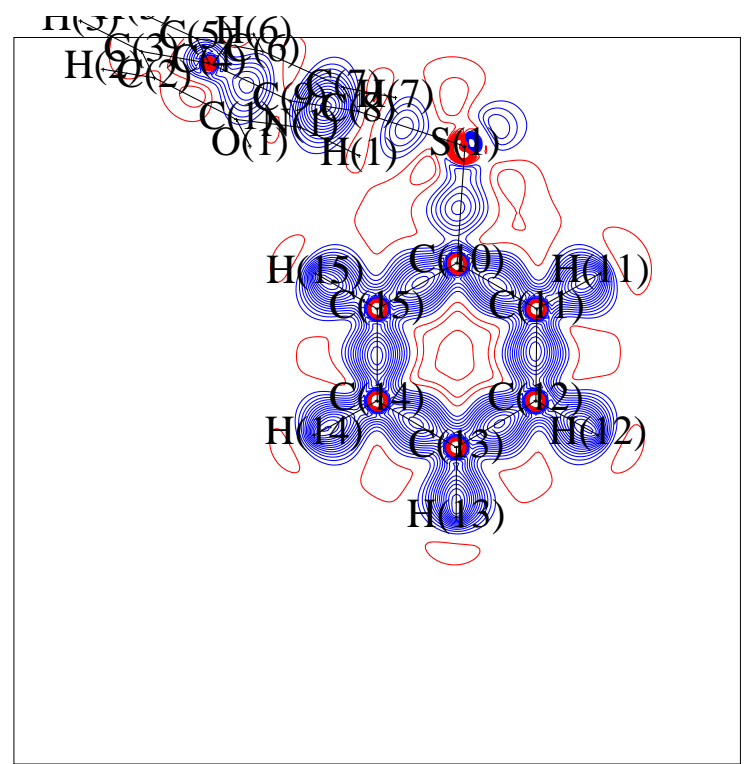

(b) XWR, harmonic, all reflections

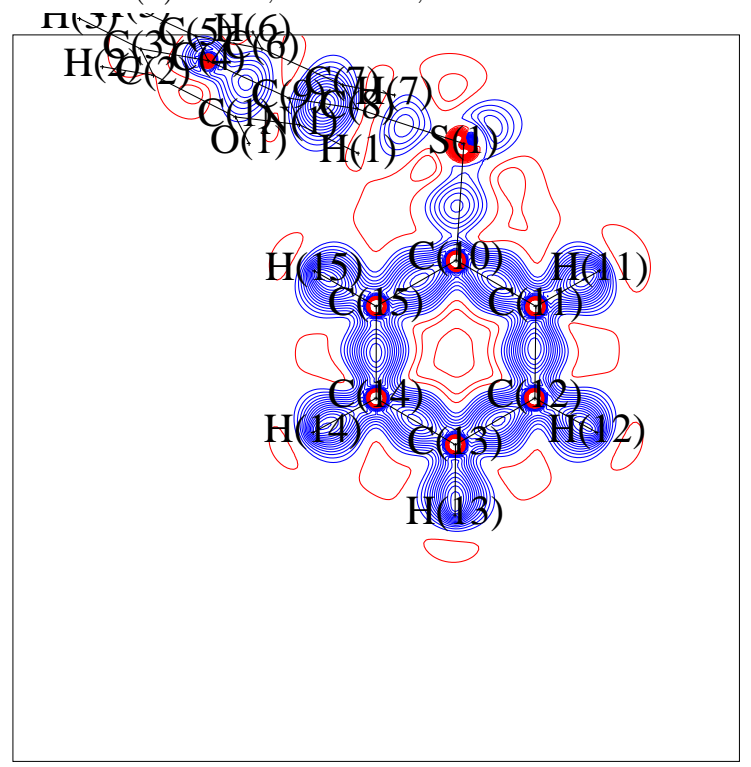

(d) XWR, anharmonic $\mathrm{n}=4$, all reflections

Figure 38: Deformation density maps for PT-11 in the plane of phenyl ring, XWR, left: $|F| \geq 2 \sigma(|F|)$ and right: all reflections included. Contour level: $0.05 \mathrm{e} / \AA^{3}$. Colours: blue - positive, red - negative. 


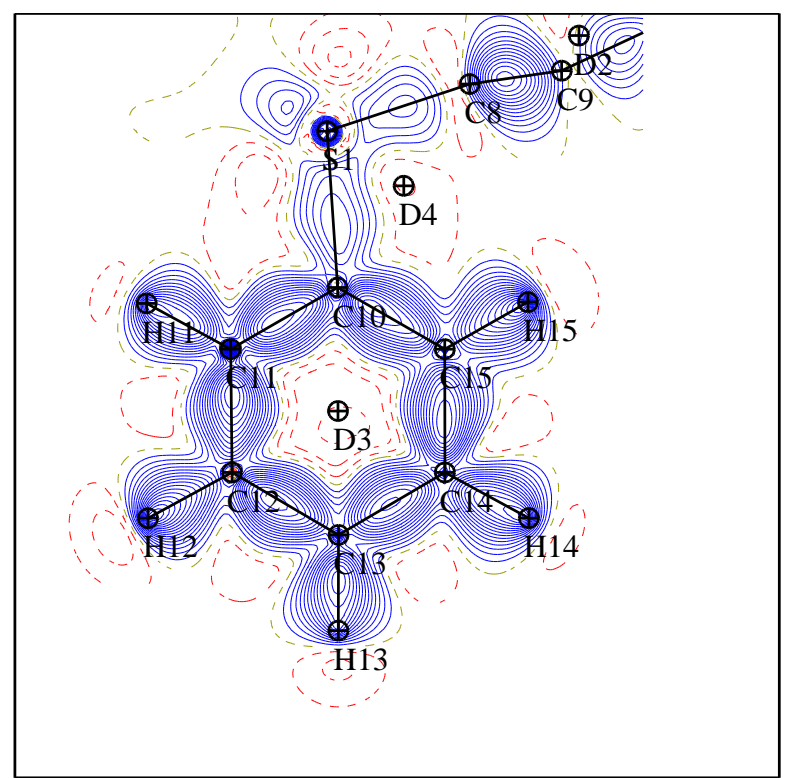

(a) MM, harmonic, $|F| \geq 2 \sigma(|F|)$

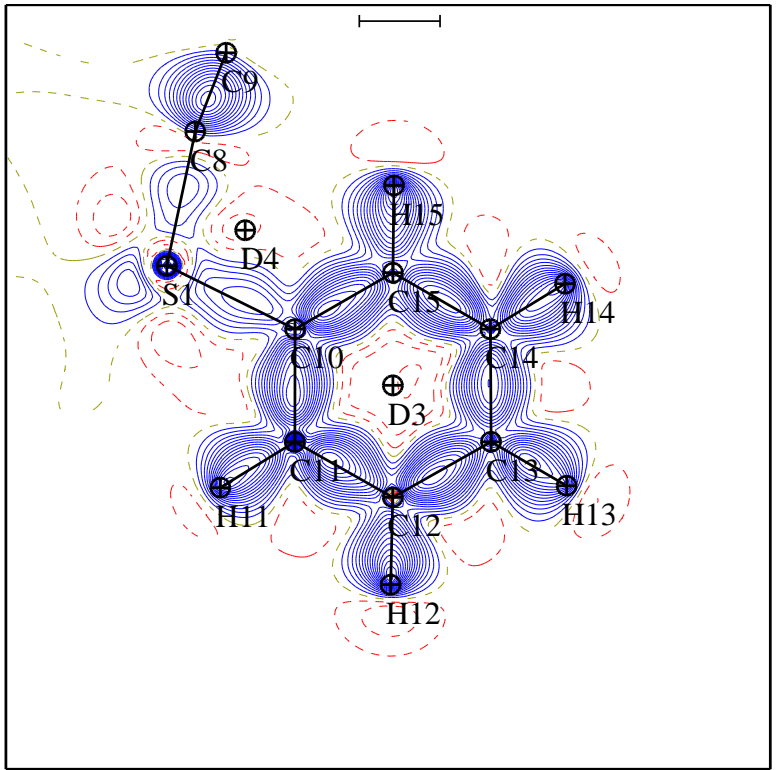

(c) MM, anharmonic $\mathrm{n}=3,|F| \geq 2 \sigma(|F|)$

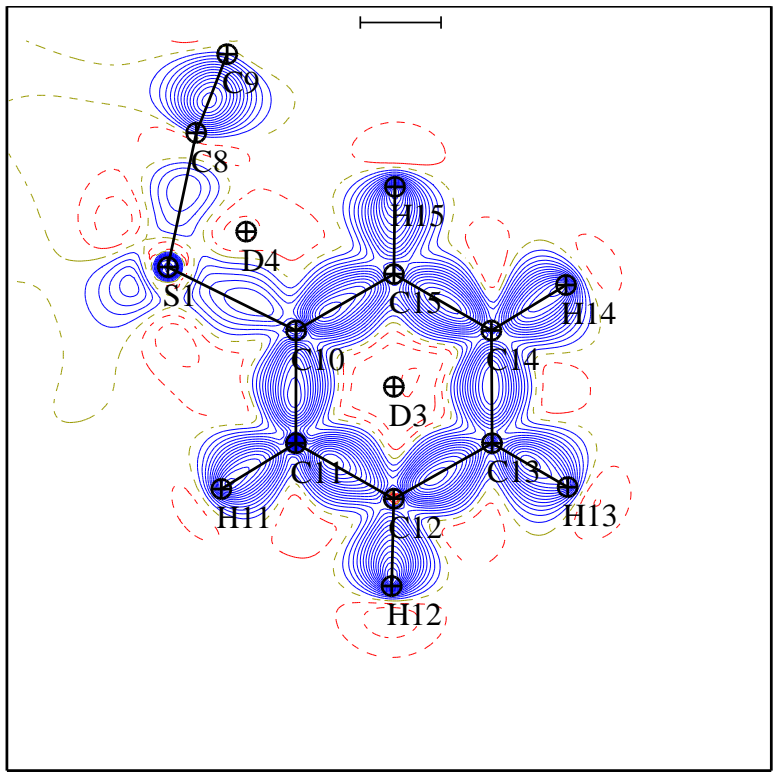

(e) MM, anharmonic $\mathrm{n}=4,|F| \geq 2 \sigma(|F|)$

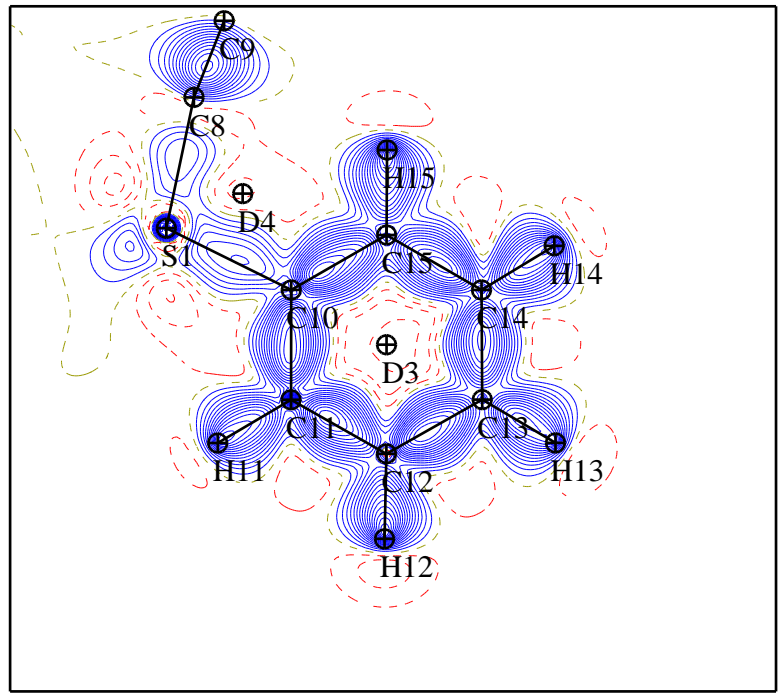

(b) MM, harmonic, all reflections

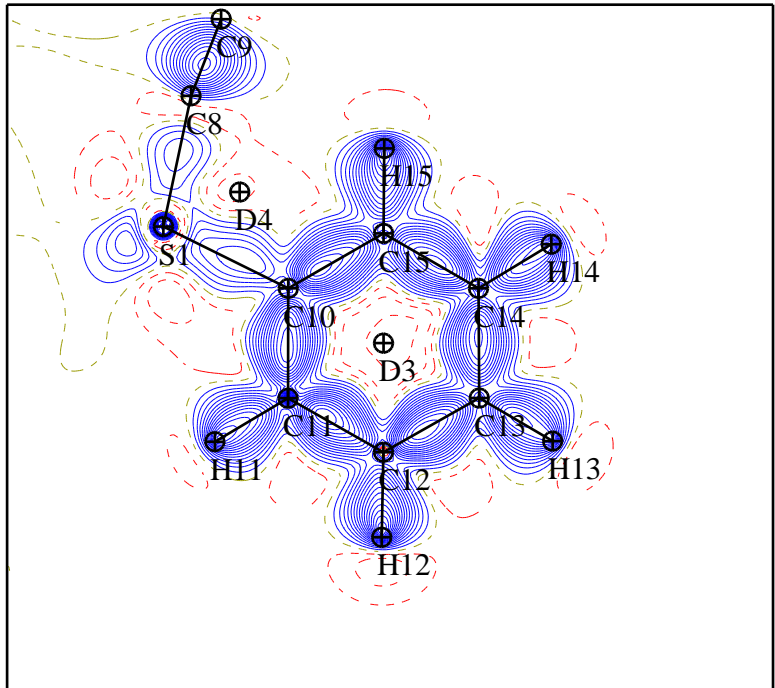

(d) $\mathrm{MM}$, anharmonic $\mathrm{n}=3$, all reflections

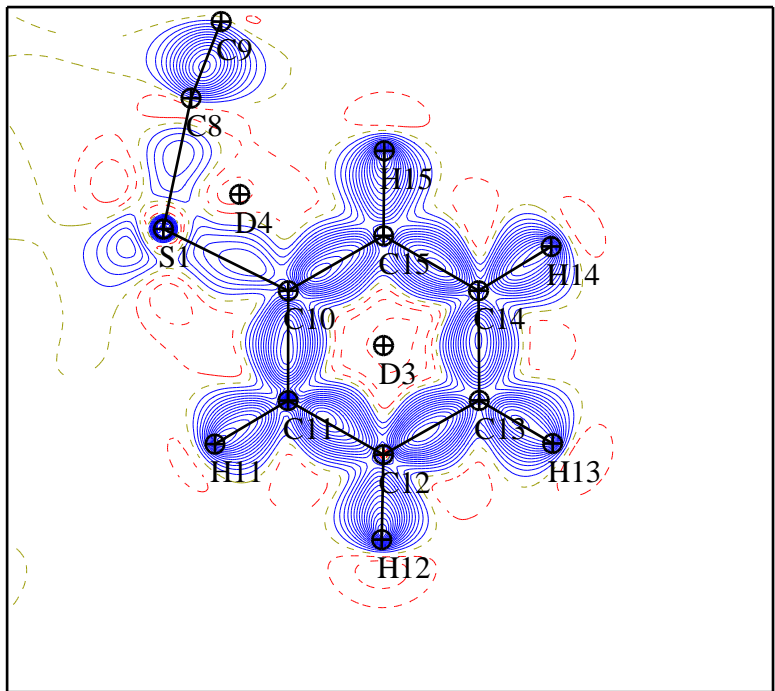

(f) MM, anharmonic $n=4$, all reflections

Figure 39: Deformation density maps for PT-11 in the plane of phenyl ring, MM, restrained X-H distances, left: $|F| \geq 2 \sigma(|F|)$ and right: all reflections included. Contour level: $0.05 \mathrm{e} / \AA^{3}$. Colours: blue - positive, red - negative. 


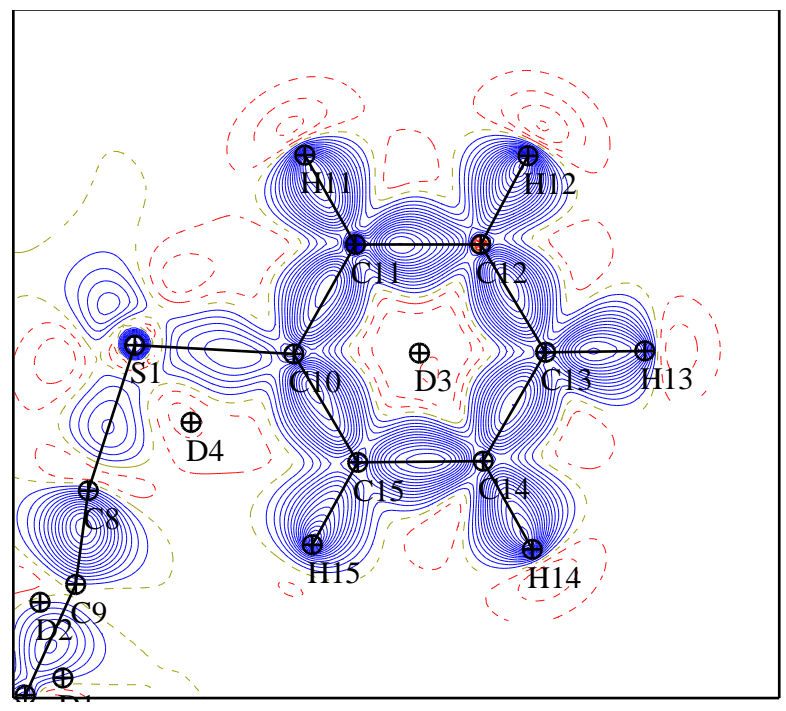

(a) MM, harmonic, $|F| \geq 2 \sigma(|F|)$

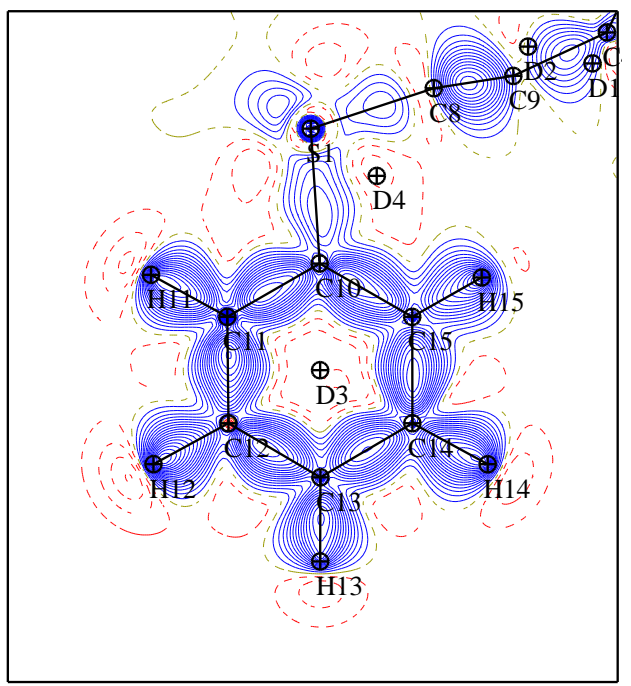

(c) MM, anharmonic n $=3,|F| \geq 2 \sigma(|F|)$

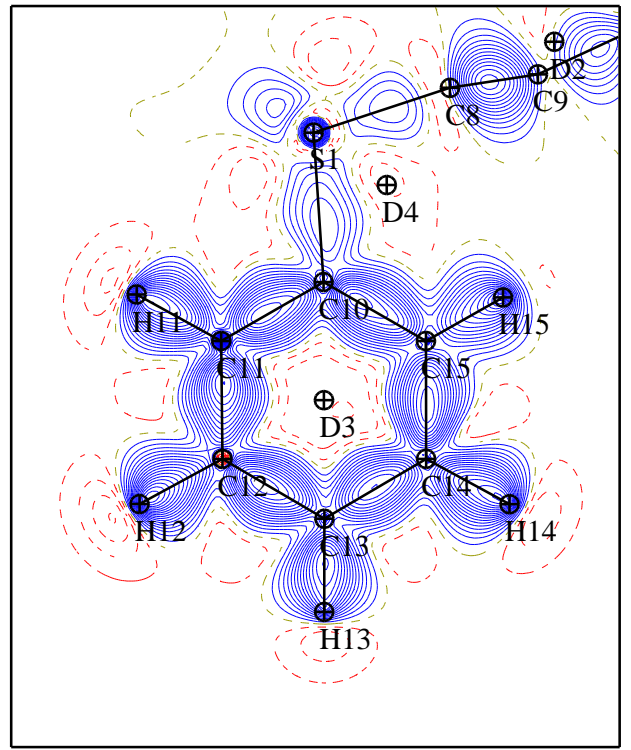

(e) MM, anharmonic $\mathrm{n}=4,|F| \geq 2 \sigma(|F|)$

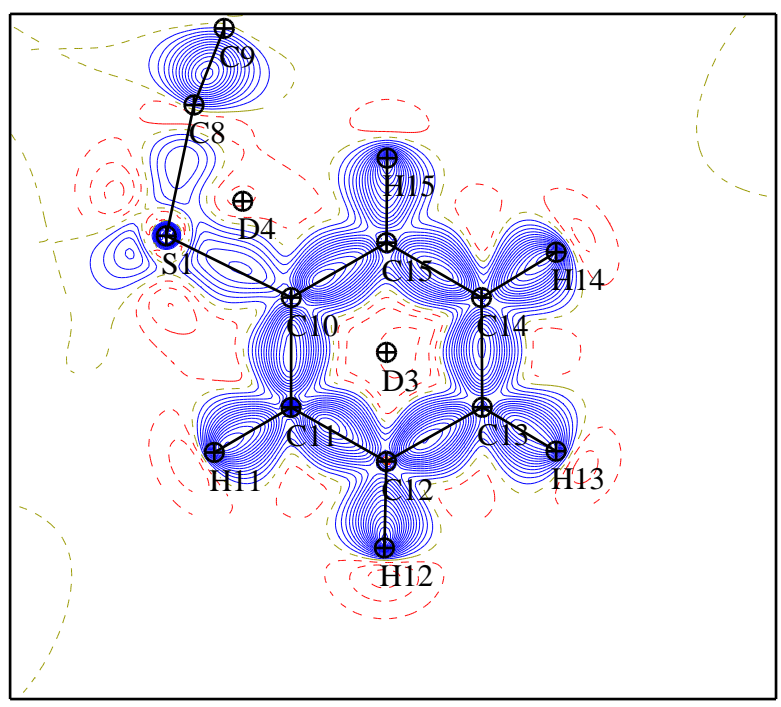

(b) MM, harmonic, all reflections

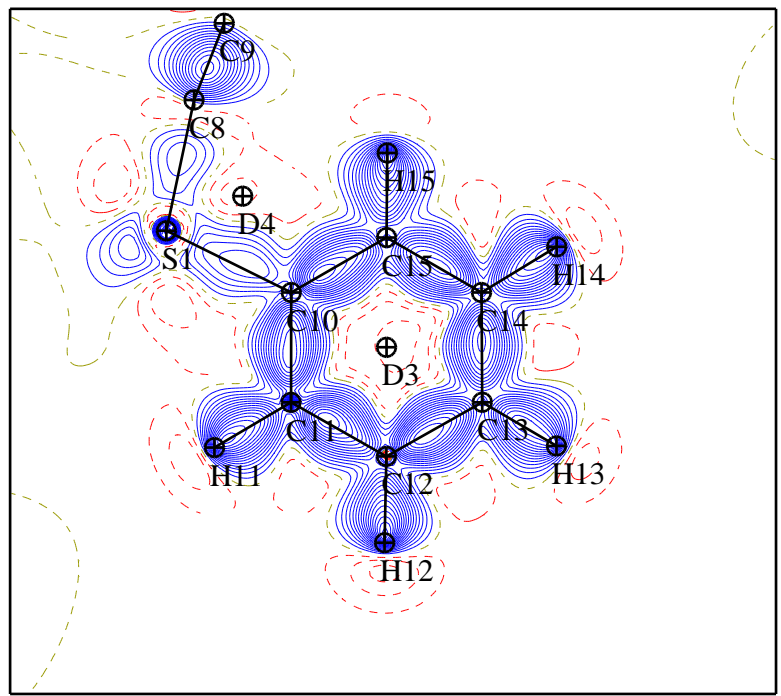

(d) MM, anharmonic $n=3$, all reflections

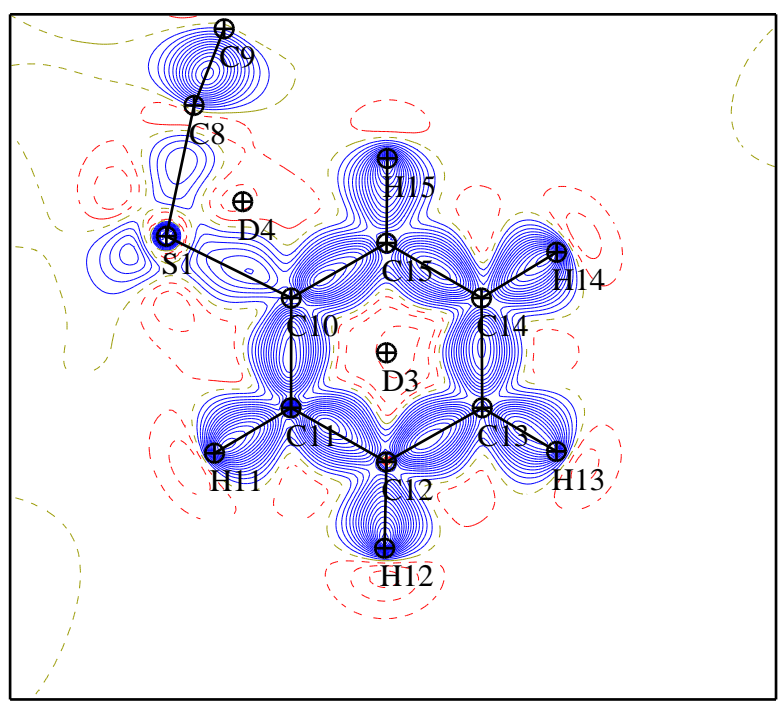

(f) MM, anharmonic $\mathrm{n}=4$, all reflections

Figure 40: Deformation density maps for PT-11 in the plane of phenyl ring, MM, unrestrained X-H distances, left: $|F| \geq 2 \sigma(|F|)$ and right: all reflections included. Contour level: $0.05 \mathrm{e} / \AA^{3}$. Colours: blue - positive, red - negative. 


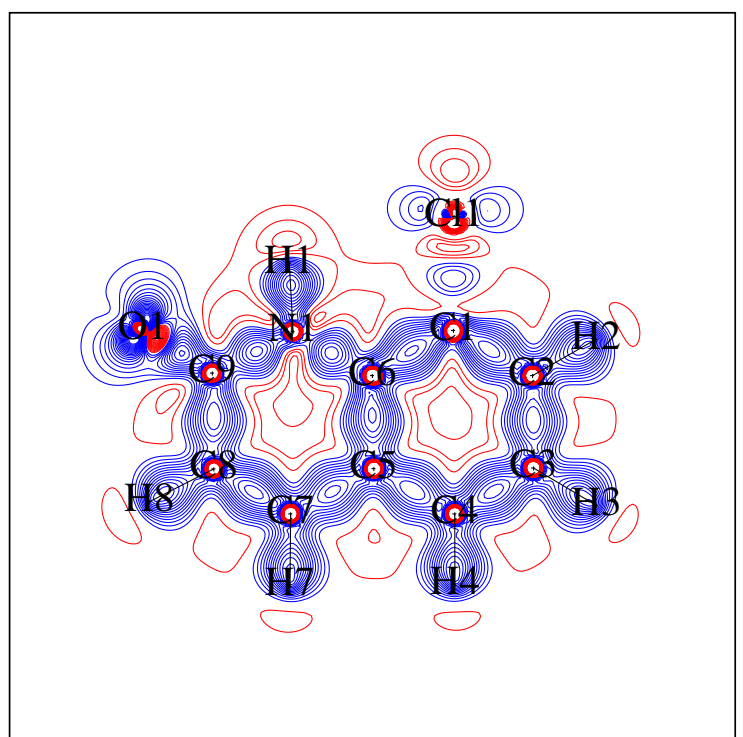

(a) HAR, harmonic, $|F| \geq 2 \sigma(|F|)$

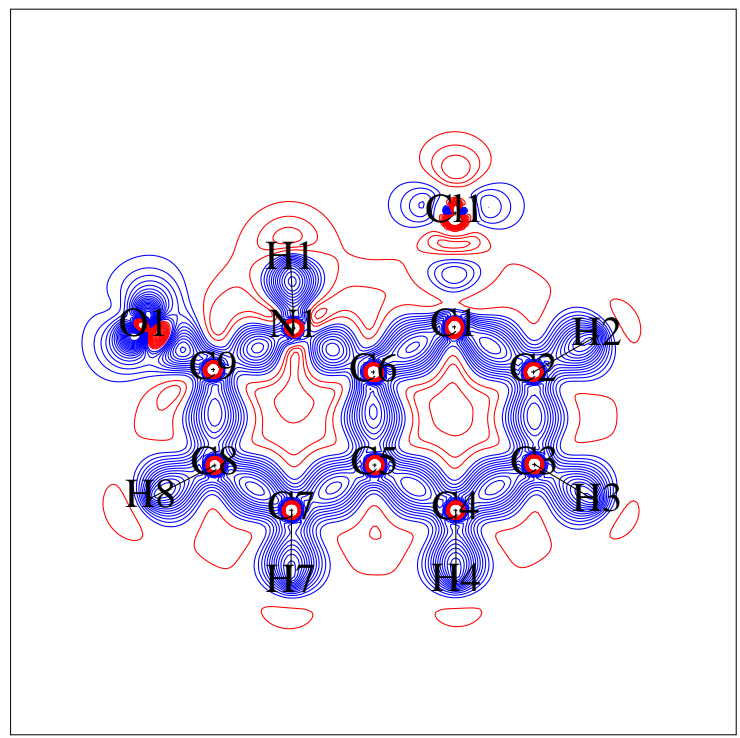

(c) HAR, anharmonic $\mathrm{n}=3,|F| \geq 2 \sigma(|F|)$

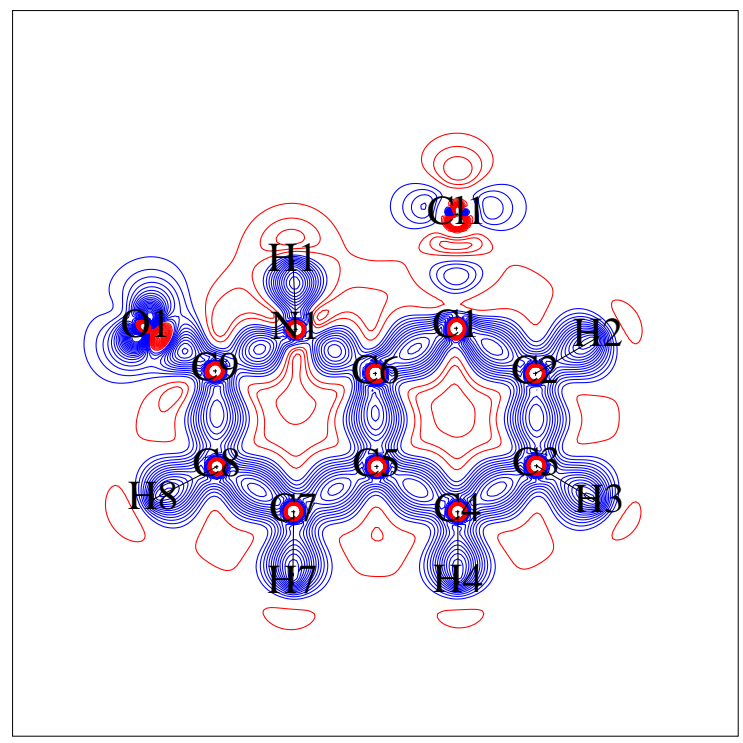

(e) HAR, anharmonic $\mathrm{n}=4,|F| \geq 2 \sigma(|F|)$

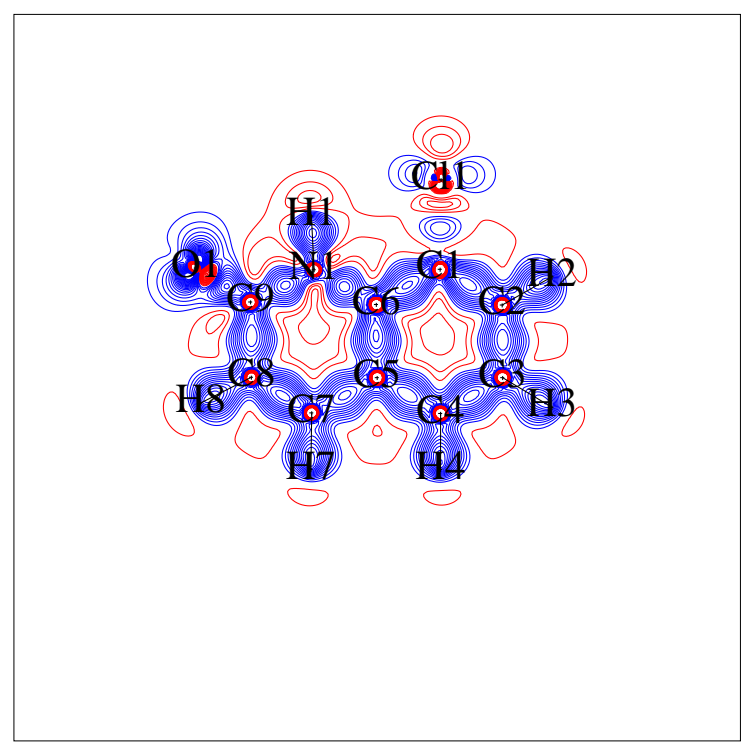

(b) HAR, harmonic, all reflections

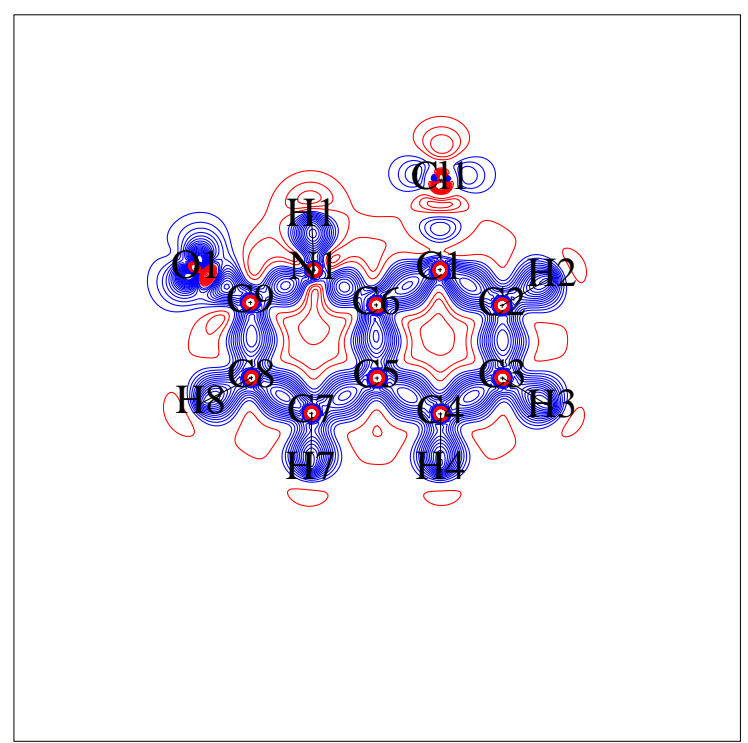

(d) HAR, anharmonic $\mathrm{n}=3$, all reflections

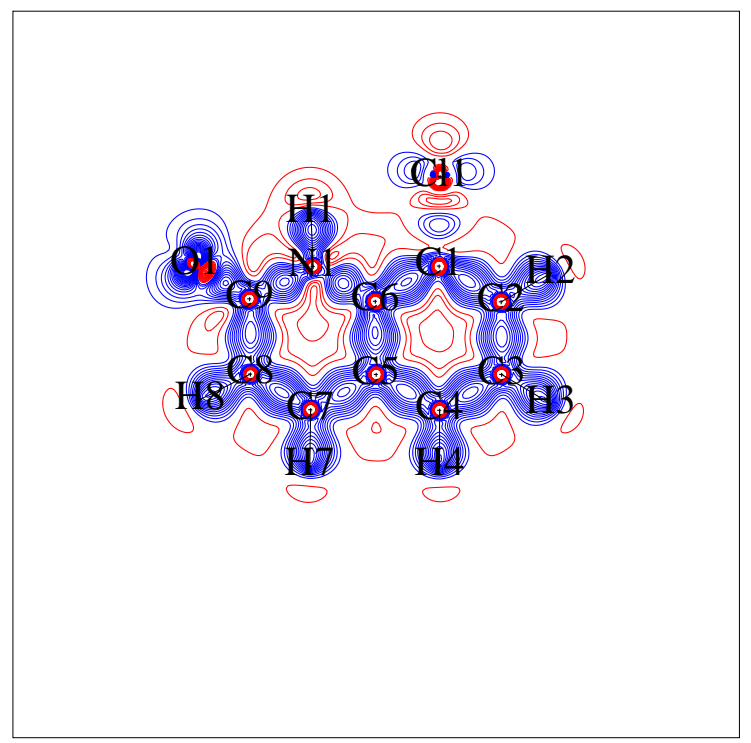

(f) HAR, anharmonic $\mathrm{n}=4$, all reflections

Figure 41: Deformation density maps for PT-2, HAR, left: $|F| \geq 2 \sigma(|F|)$ and right: all reflections included. Contour level: 0.05 e/ $\AA^{3}$. Colours: blue - positive, red - negative. 


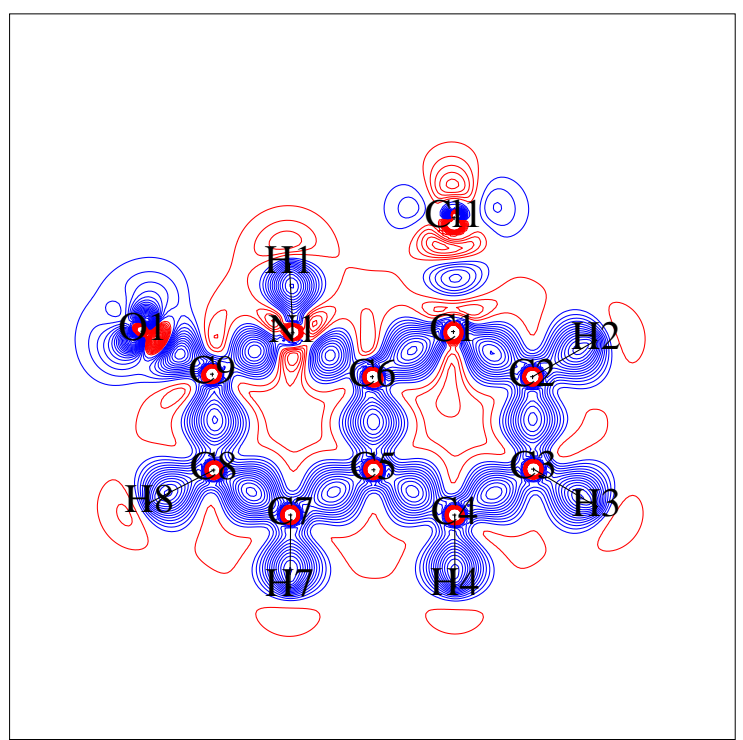

(a) XWR, harmonic, $|F| \geq 2 \sigma(|F|)$

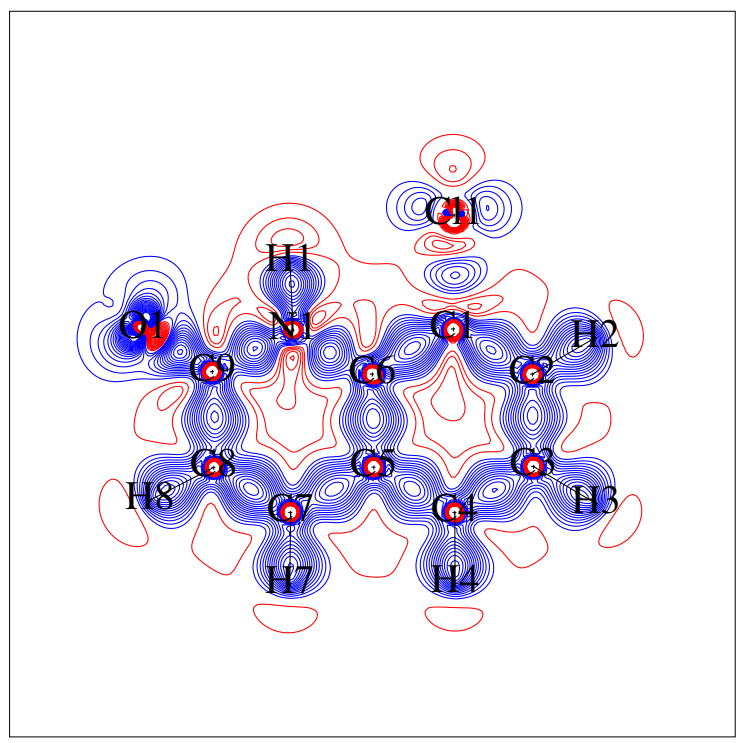

(c) XWR, anharmonic $\mathrm{n}=4,|F| \geq 2 \sigma(|F|)$

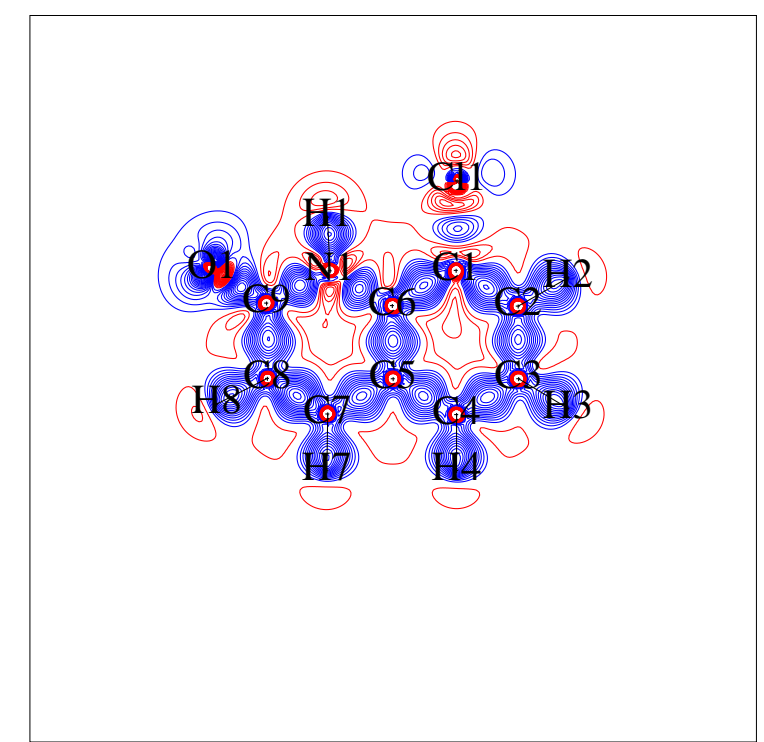

(b) XWR, harmonic, all reflections

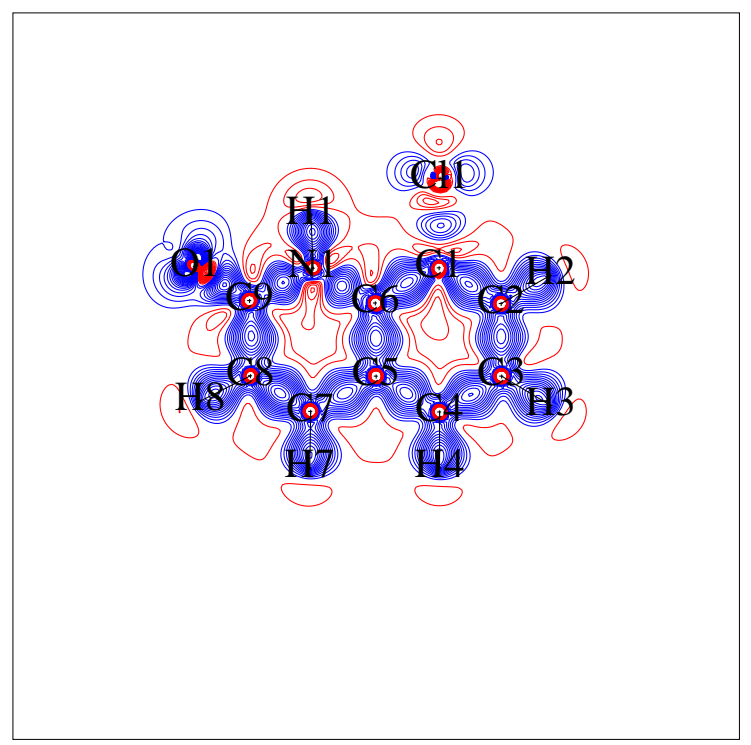

(d) XWR, anharmonic $\mathrm{n}=4$, all reflections

Figure 42: Deformation density maps for PT-2, XWR, left: $|F| \geq 2 \sigma(|F|)$ and right: all reflections included. Contour level: 0.05 e/ $\AA^{3}$. Colours: blue - positive, red - negative. 


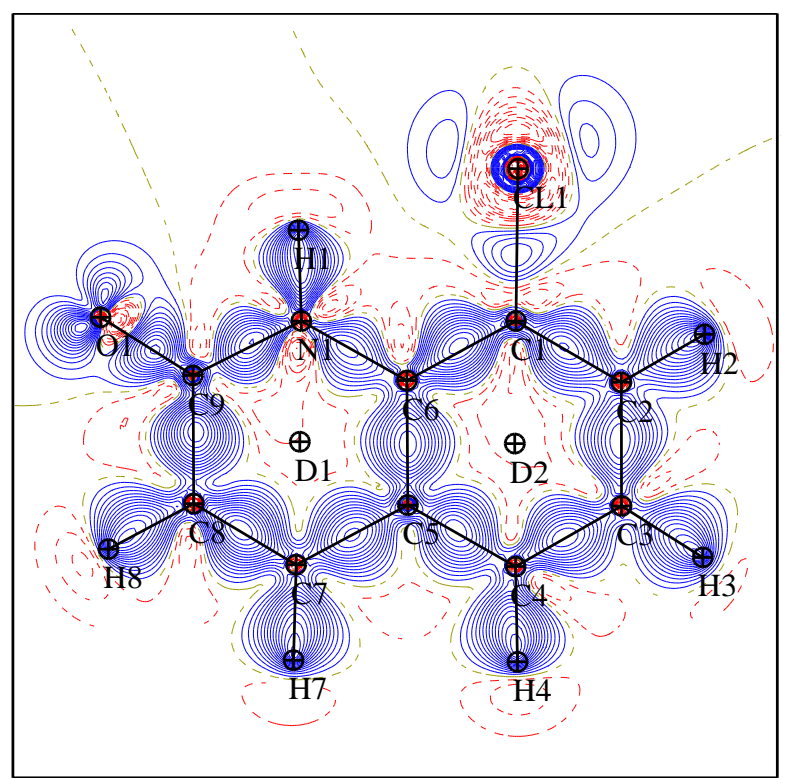

(a) MM, harmonic, $|F| \geq 2 \sigma(|F|)$

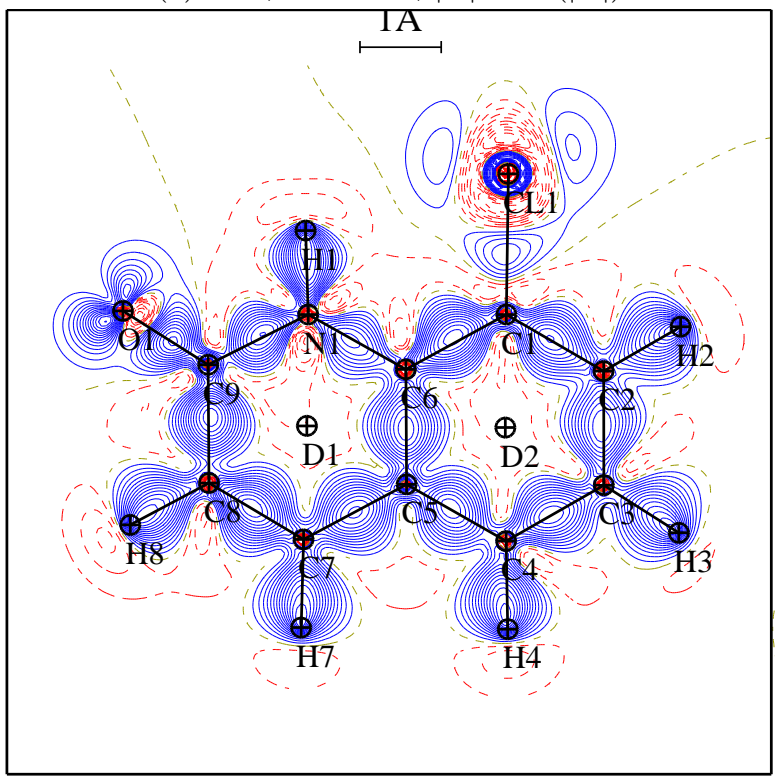

(c) MM, anharmonic $\mathrm{n}=3,|F| \geq 2 \sigma(|F|)$

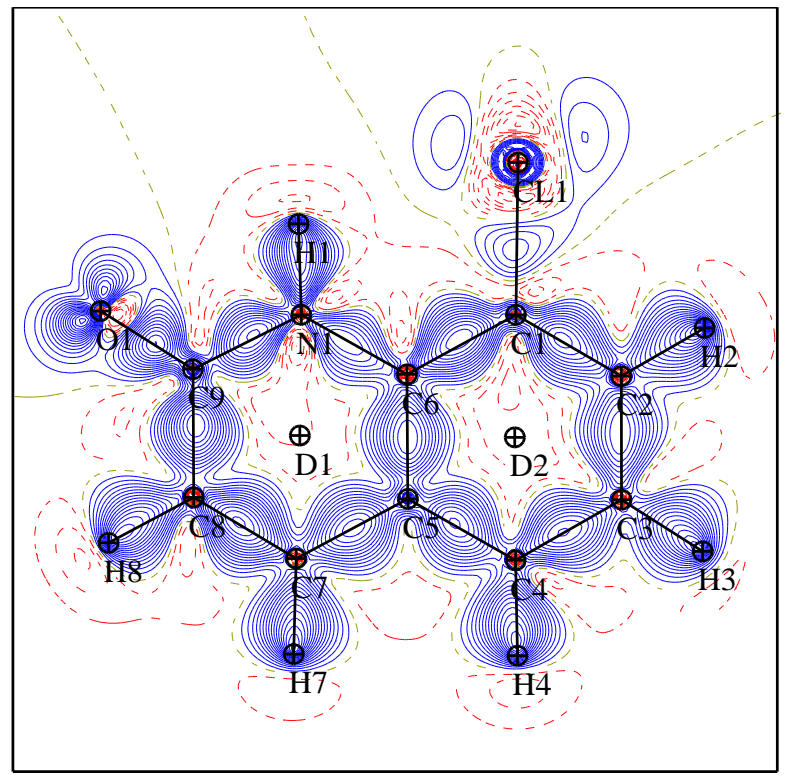

(e) MM, anharmonic $\mathrm{n}=4,|F| \geq 2 \sigma(|F|)$

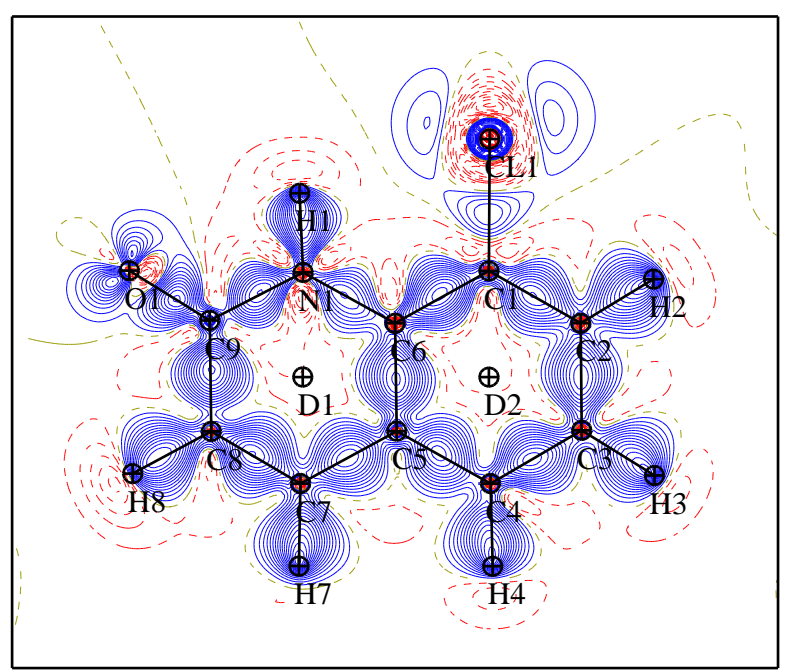

(b) MM, harmonic, all reflections

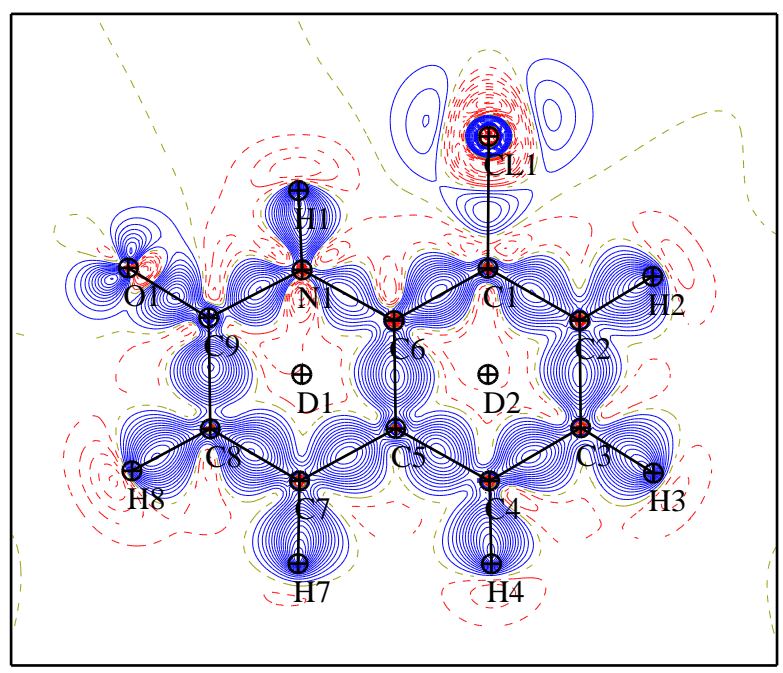

(d) MM, anharmonic $\mathrm{n}=3$, all reflections

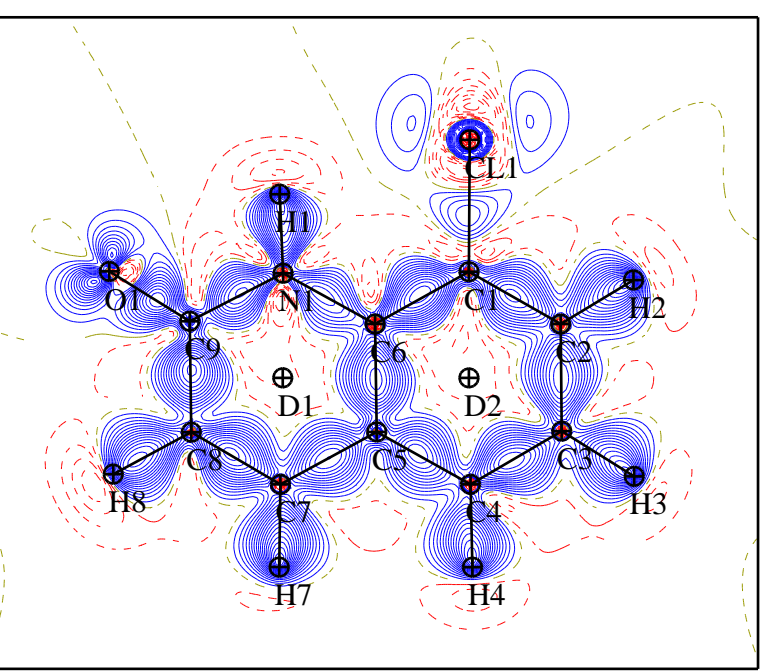

(f) MM, anharmonic $\mathrm{n}=4$, all reflections

Figure 43: Deformation density maps for PT-2, MM, restrained X-H distances, left: $|F| \geq 2 \sigma(|F|)$ and right: all reflections included. Contour level: $0.05 \mathrm{e} / \AA^{3}$. Colours: blue - positive, red - negative. 


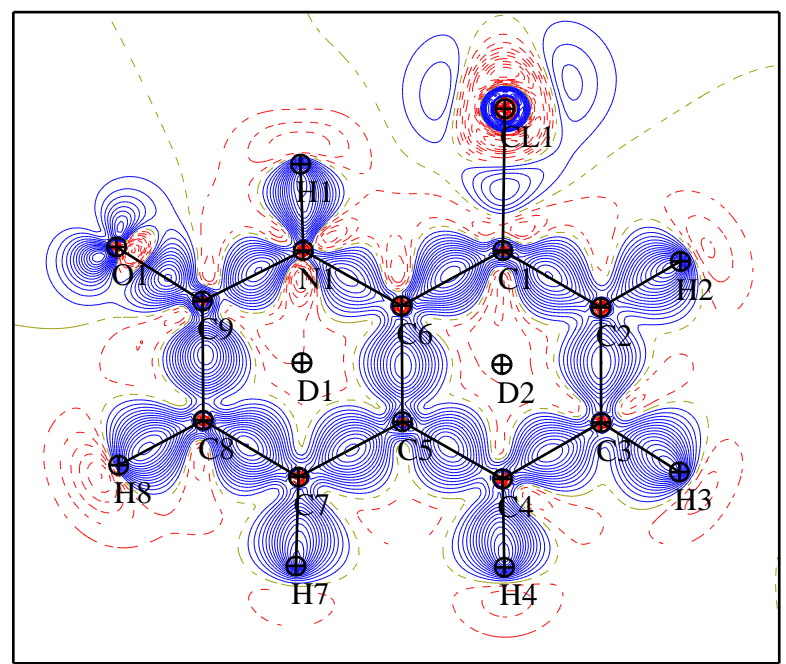

(a) MM, harmonic, $|F| \geq 2 \sigma(|F|)$

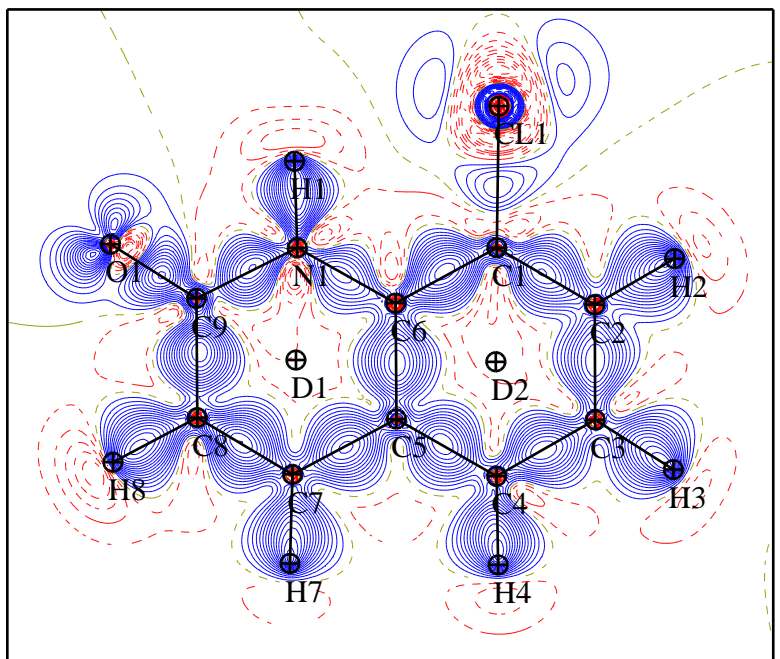

(c) MM, anharmonic $\mathrm{n}=3,|F| \geq 2 \sigma(|F|)$

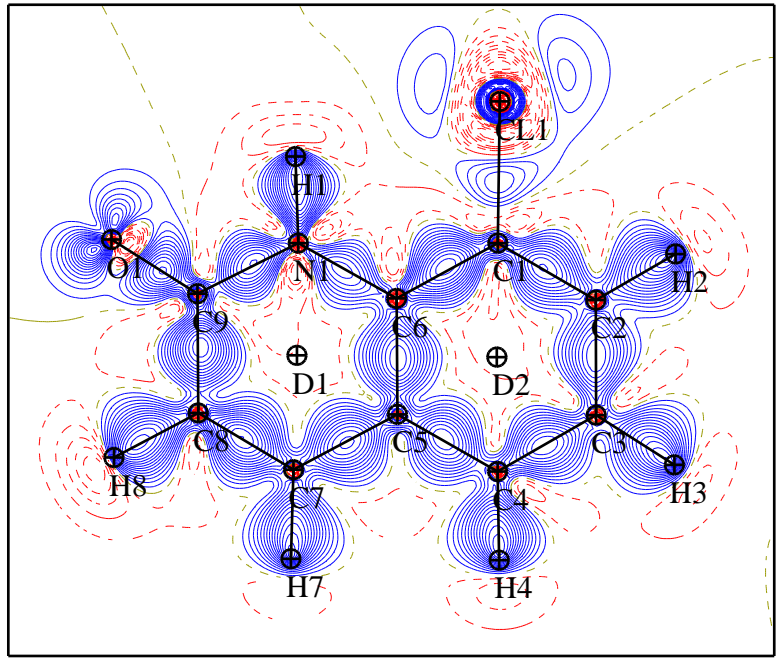

(e) MM, anharmonic $\mathrm{n}=4,|F| \geq 2 \sigma(|F|)$

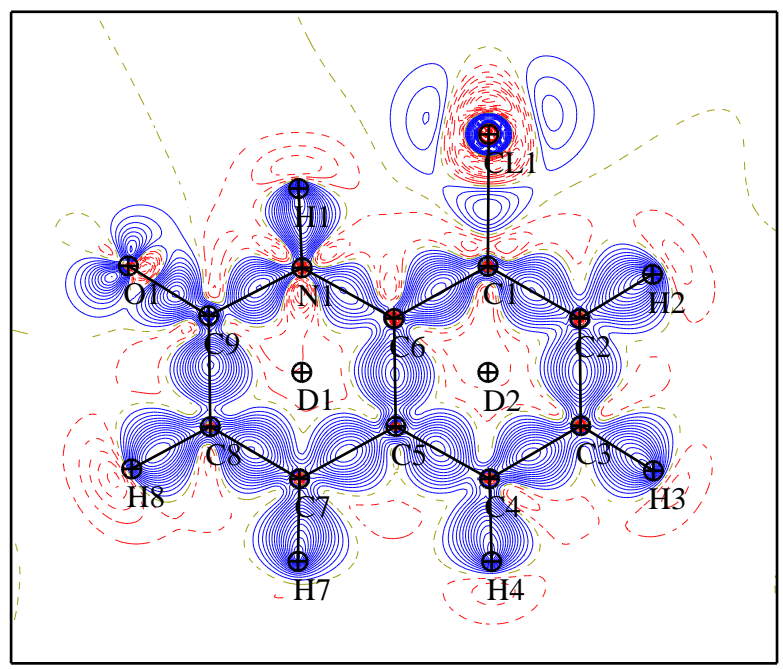

(b) MM, harmonic, all reflections

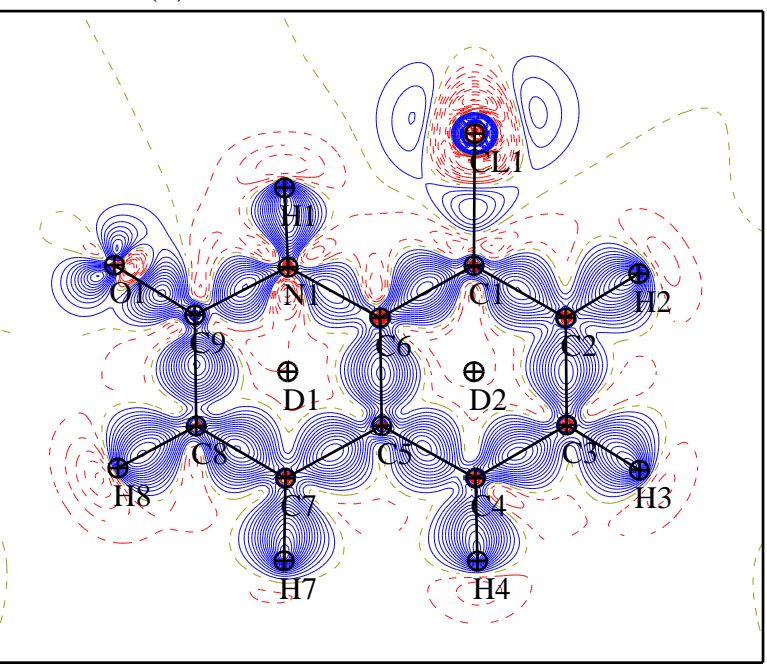

(d) MM, anharmonic $\mathrm{n}=3$, all reflections

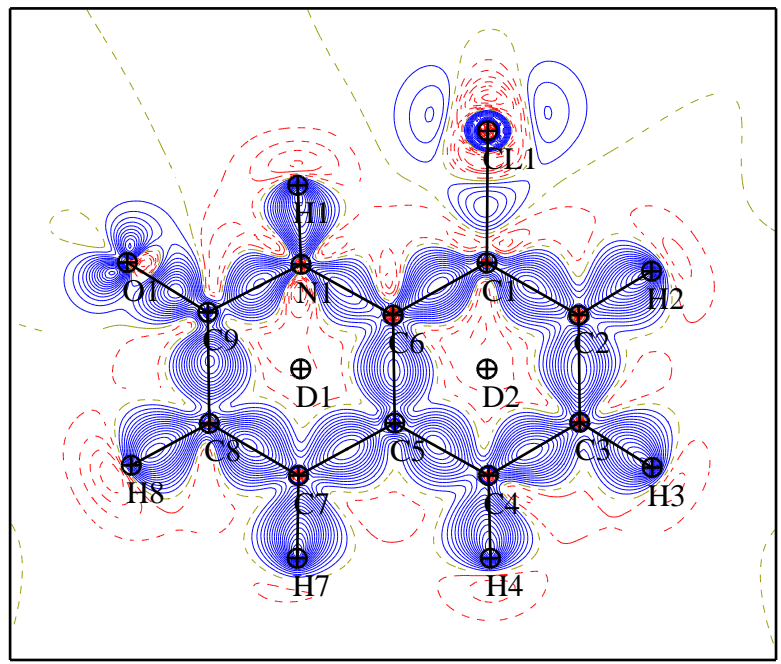

(f) $M M$, anharmonic $n=4$, all reflections

Figure 44: Deformation density maps for PT-2, MM, unrestrained X-H distances, left: $|F| \geq 2 \sigma(|F|)$ and right: all reflections included. Contour level: $0.05 \mathrm{e} / \AA^{3}$. Colours: blue - positive, red - negative. 


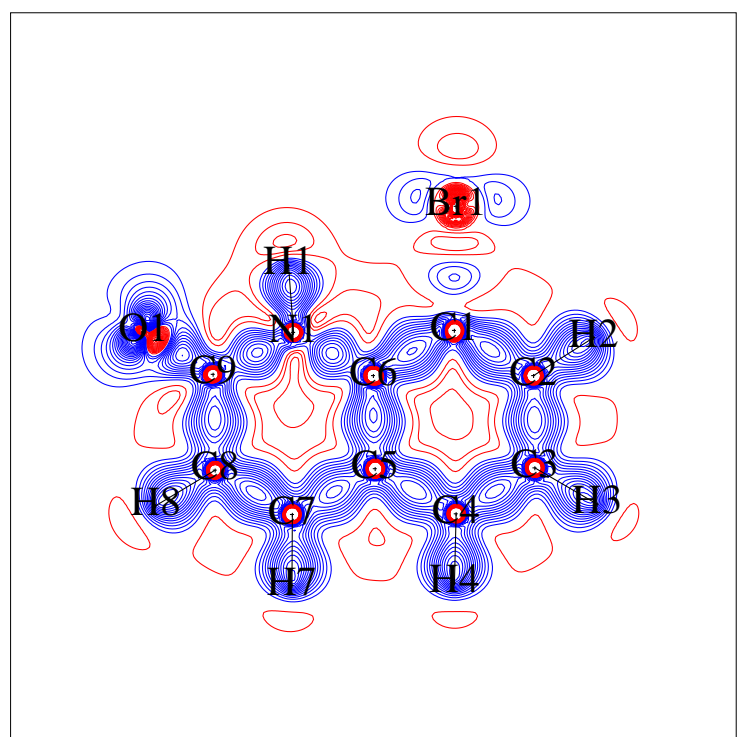

(a) HAR, harmonic, $|F| \geq 2 \sigma(|F|)$

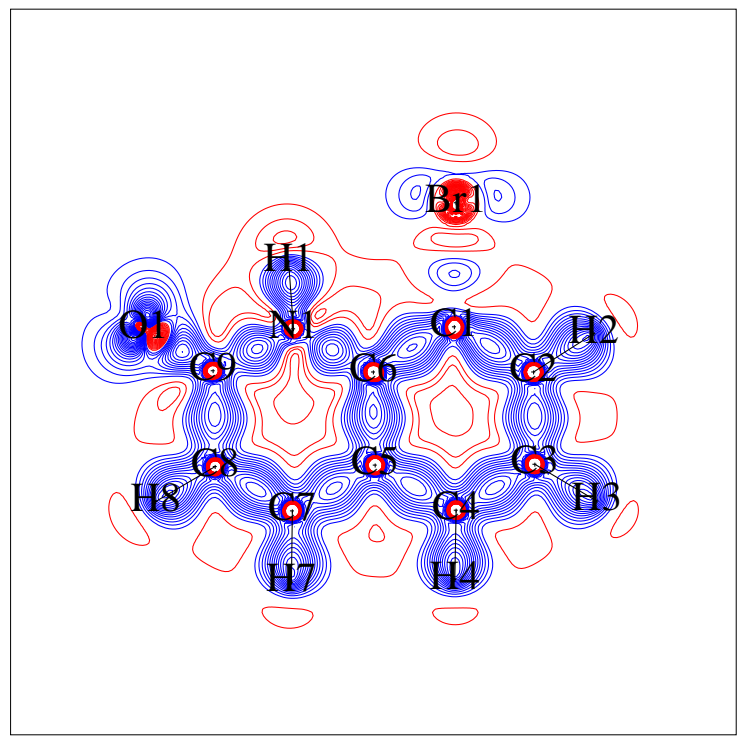

(c) HAR, anharmonic n $=3,|F| \geq 2 \sigma(|F|)$

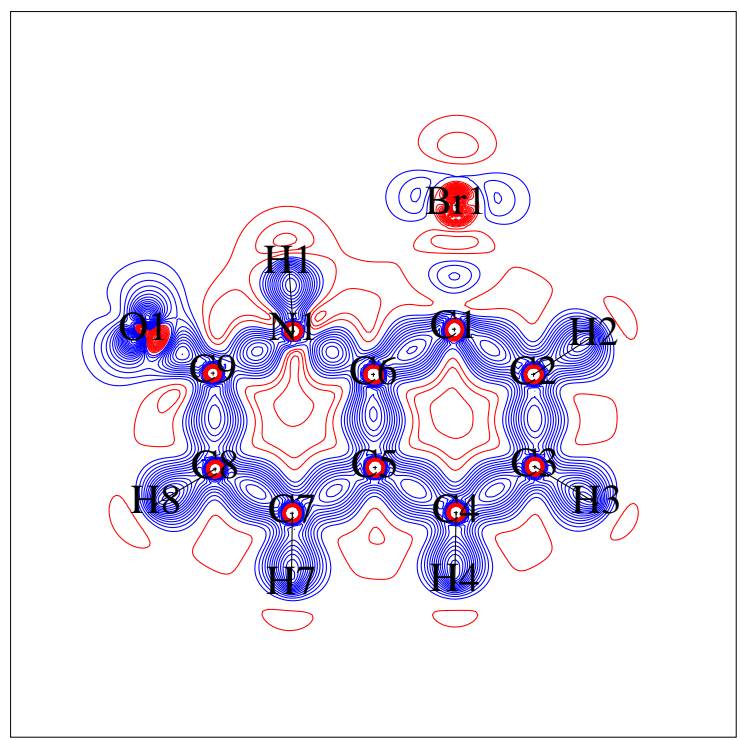

(e) HAR, anharmonic n=4, $|F| \geq 2 \sigma(|F|)$

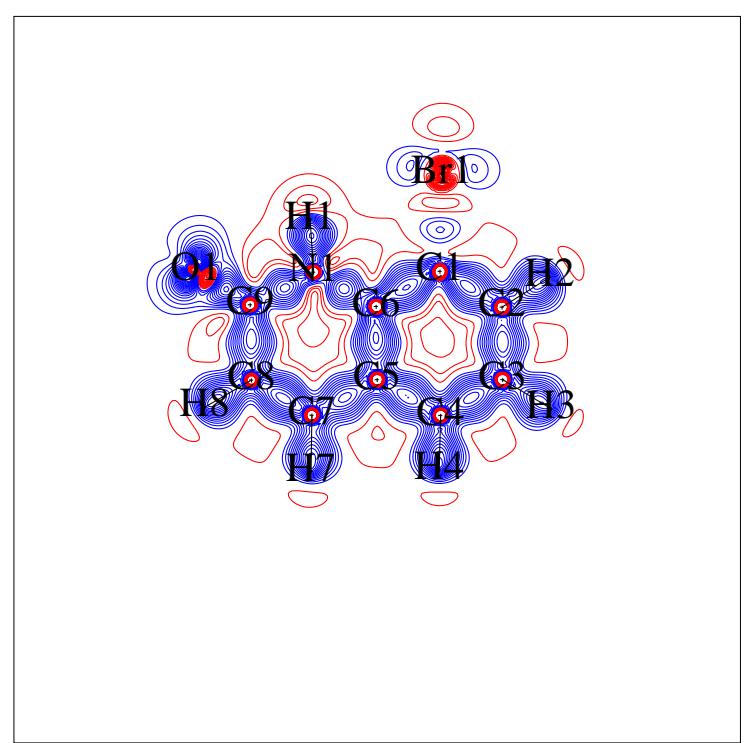

(b) HAR, harmonic, all reflections

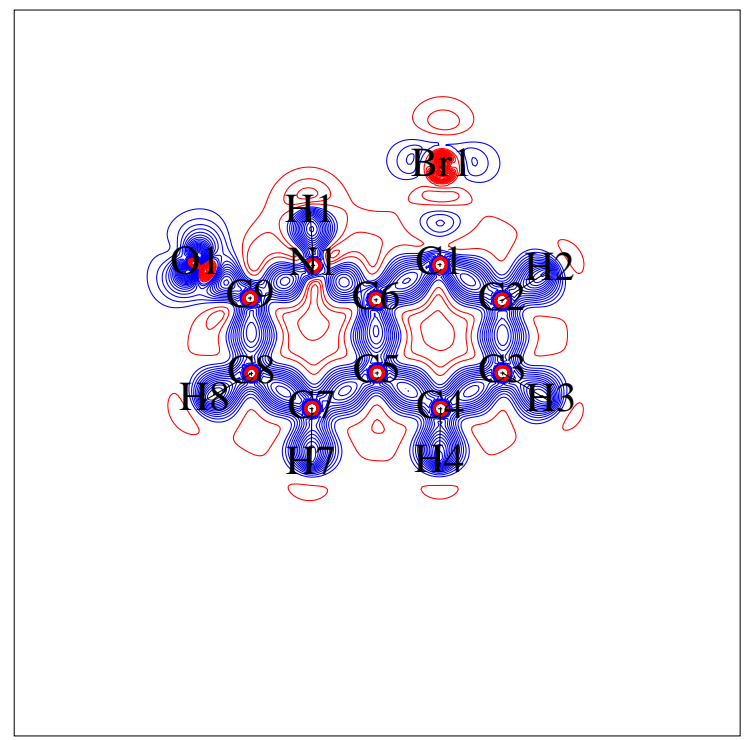

(d) HAR, anharmonic $n=3$, all reflections

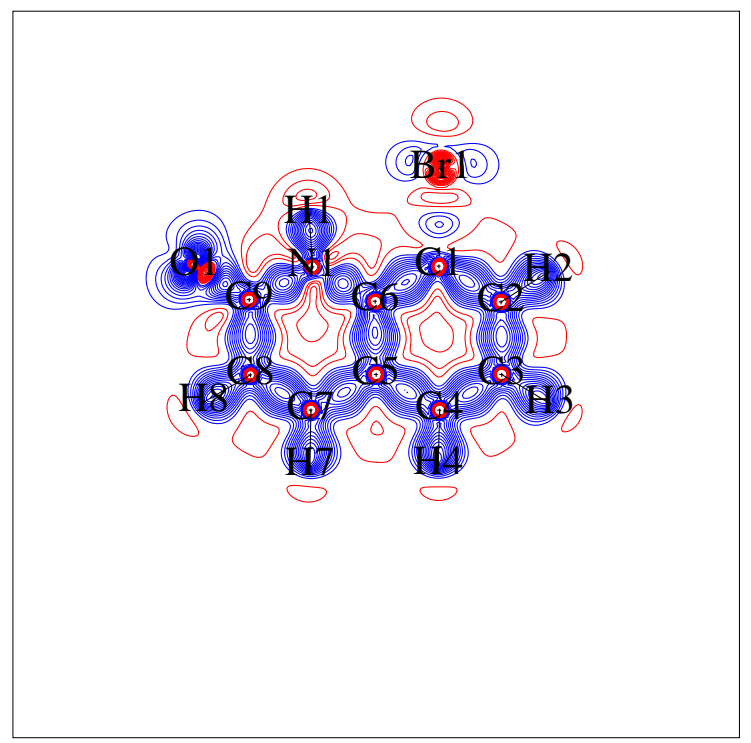

(f) HAR, anharmonic $\mathrm{n}=4$, all reflections

Figure 45: Deformation density maps for PT-8, HAR, left: $|F| \geq 2 \sigma(|F|)$ and right: all reflections included. Contour level: 0.05 $\mathrm{e} / \AA^{3}$. Colours: blue - positive, red - negative. 


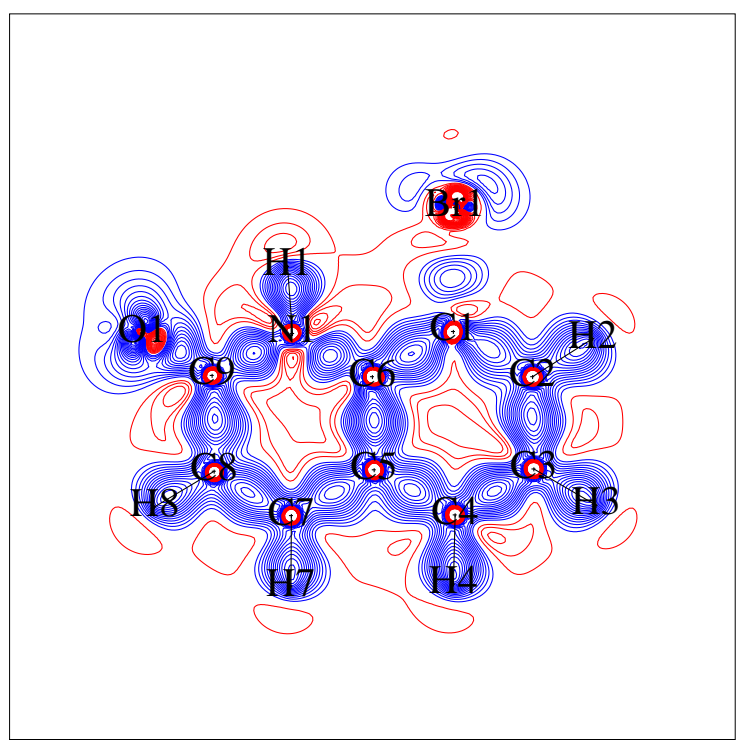

(a) XWR, harmonic, $|F| \geq 2 \sigma(|F|)$

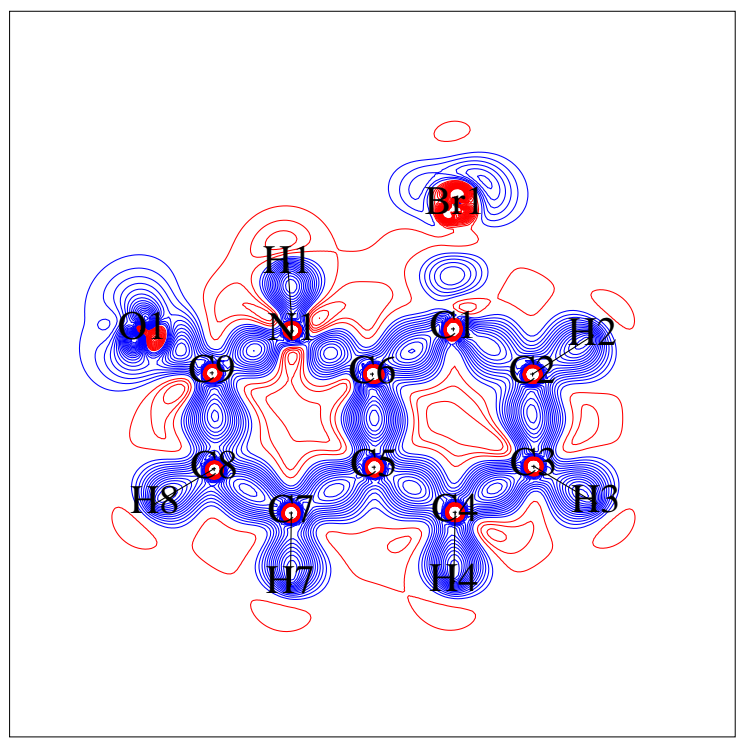

(c) XWR, anharmonic $\mathrm{n}=4,|F| \geq 2 \sigma(|F|)$

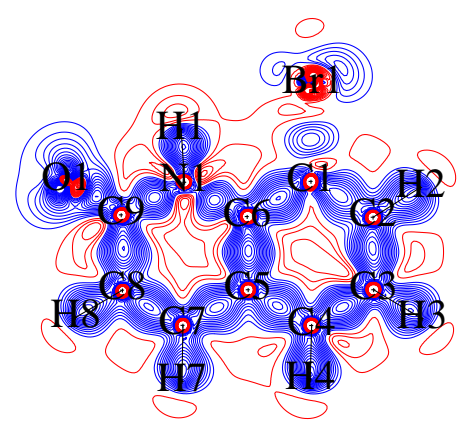

(b) XWR, harmonic, all reflections

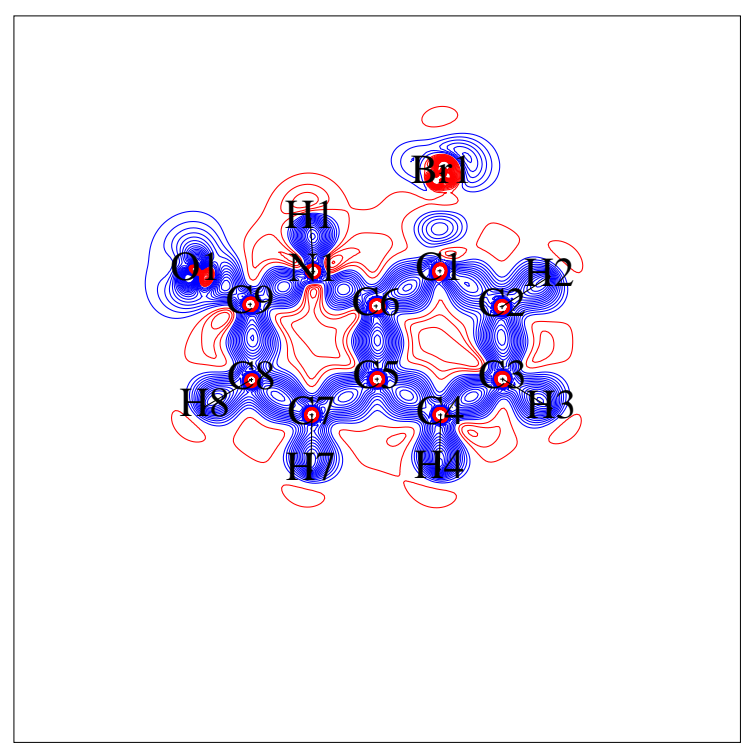

(d) XWR, anharmonic $\mathrm{n}=4$, all reflections

Figure 46: Deformation density maps for PT-8, XWR, left: $|F| \geq 2 \sigma(|F|)$ and right: all reflections included. Contour level: 0.05 $\mathrm{e} / \AA^{3}$. Colours: blue - positive, red - negative. 


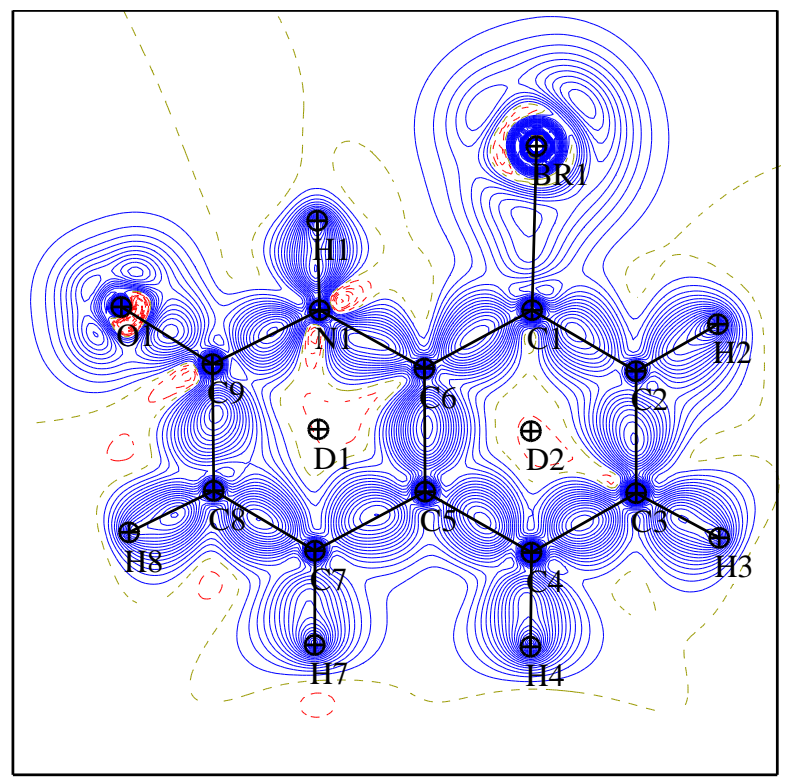

(a) MM, harmonic, $|F| \geq 2 \sigma(|F|)$

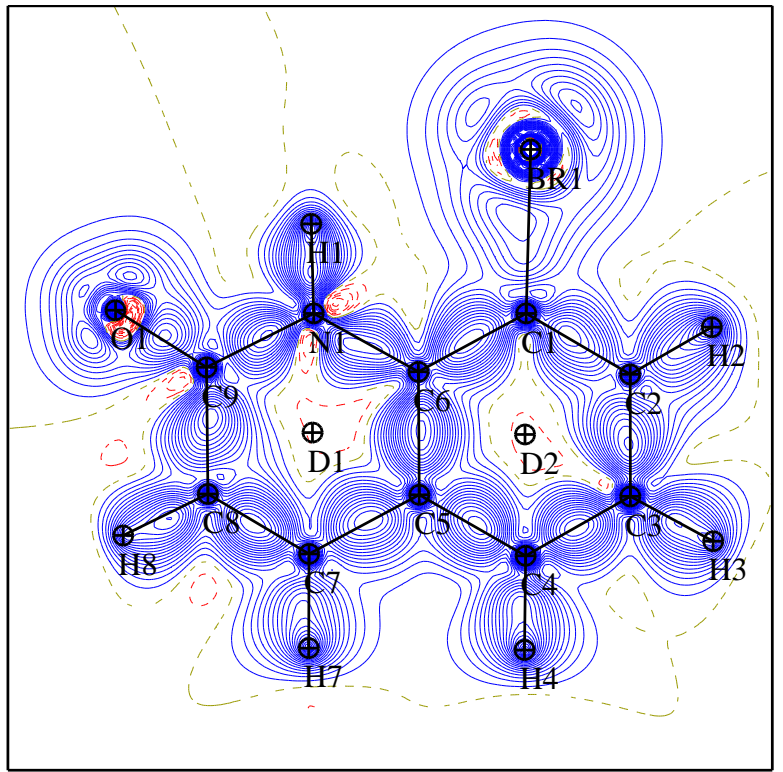

(c) MM, anharmonic $\mathrm{n}=3,|F| \geq 2 \sigma(|F|)$

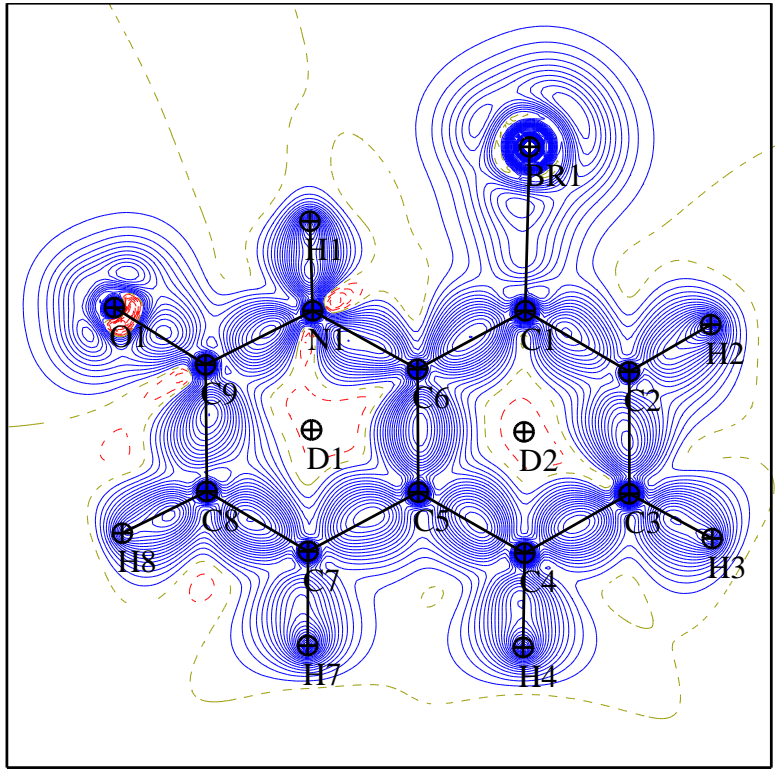

(e) MM, anharmonic n=4, $|F| \geq 2 \sigma(|F|)$

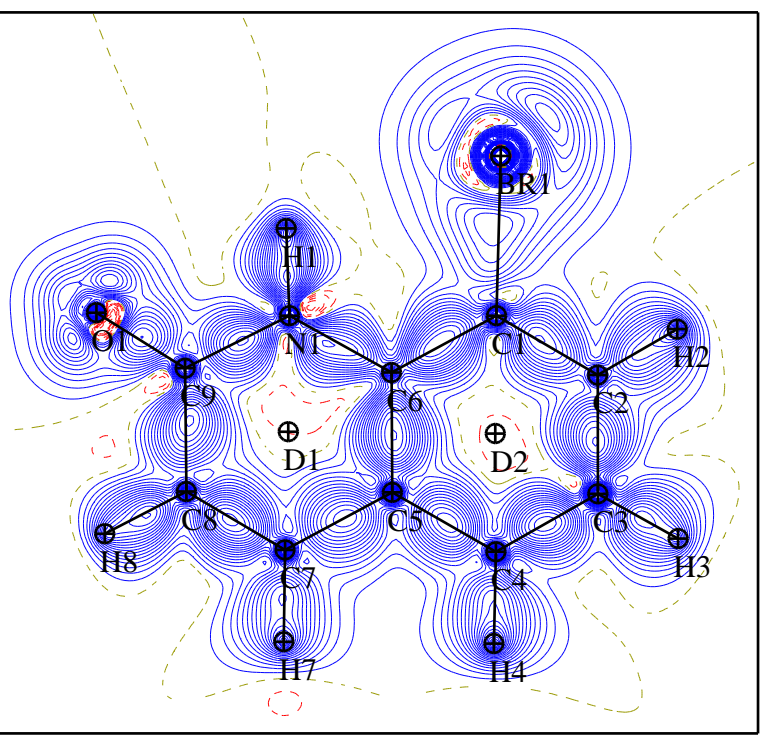

(b) MM, harmonic, all reflections

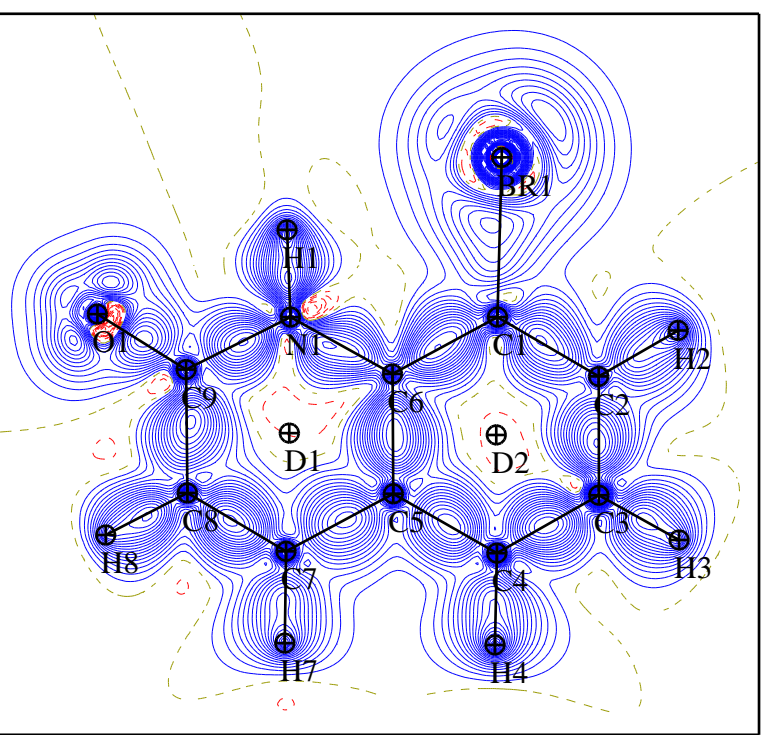

(d) MM, anharmonic $\mathrm{n}=3$, all reflections

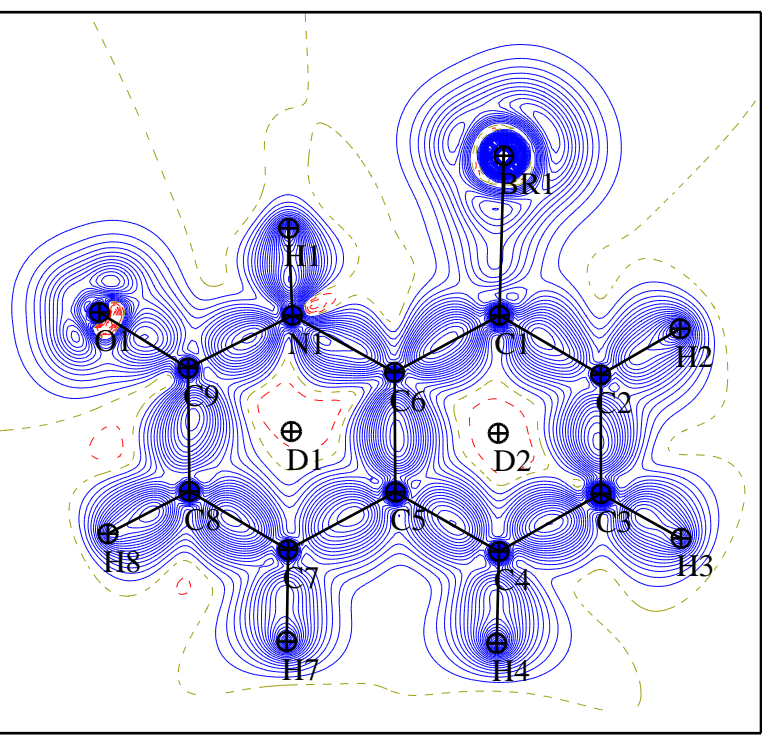

(f) MM, anharmonic $\mathrm{n}=4$, all reflections

Figure 47: Deformation density maps for PT-8, MM, restrained X-H distances, left: $|F| \geq 2 \sigma(|F|)$ and right: all reflections included. Contour level: $0.05 \mathrm{e} / \AA^{3}$. Colours: blue - positive, red - negative. 


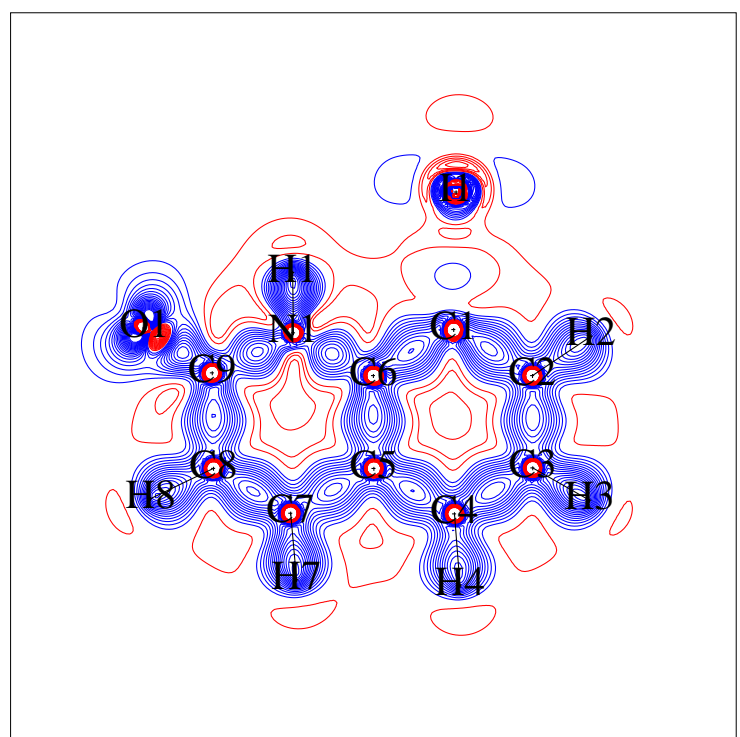

(a) HAR, harmonic, $|F| \geq 2 \sigma(|F|)$

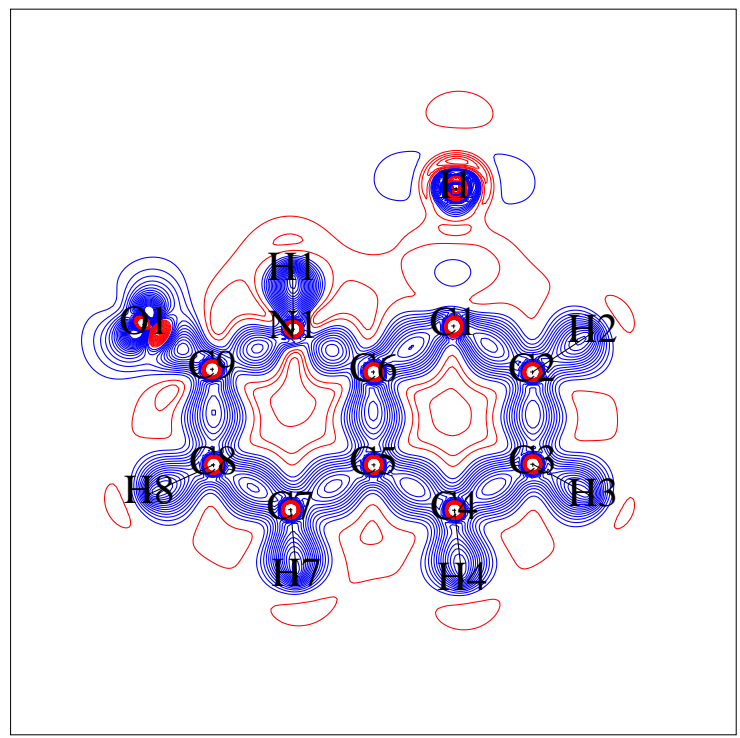

(c) HAR, anharmonic $\mathrm{n}=3,|F| \geq 2 \sigma(|F|)$

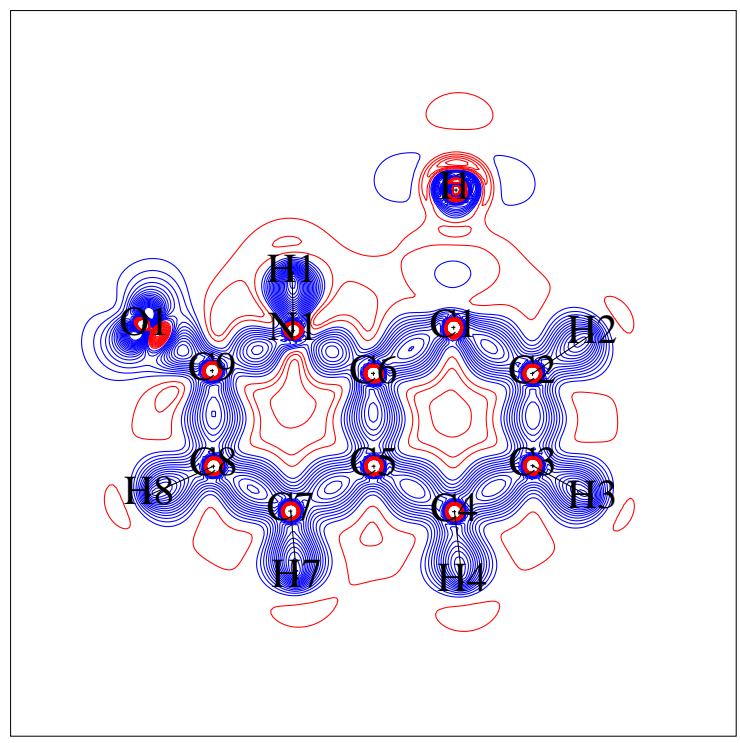

(e) HAR, anharmonic $\mathrm{n}=4,|F| \geq 2 \sigma(|F|)$

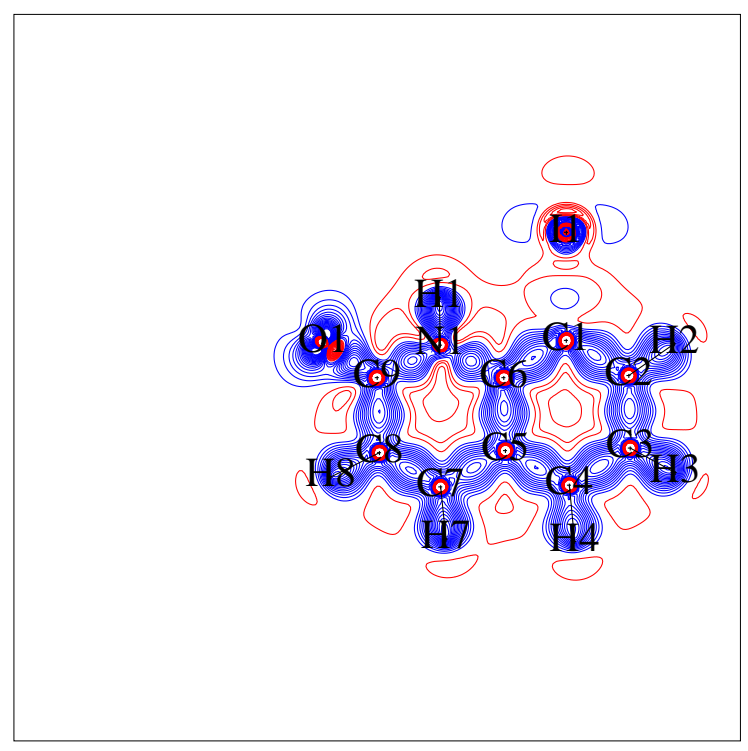

(b) HAR, harmonic, all reflections

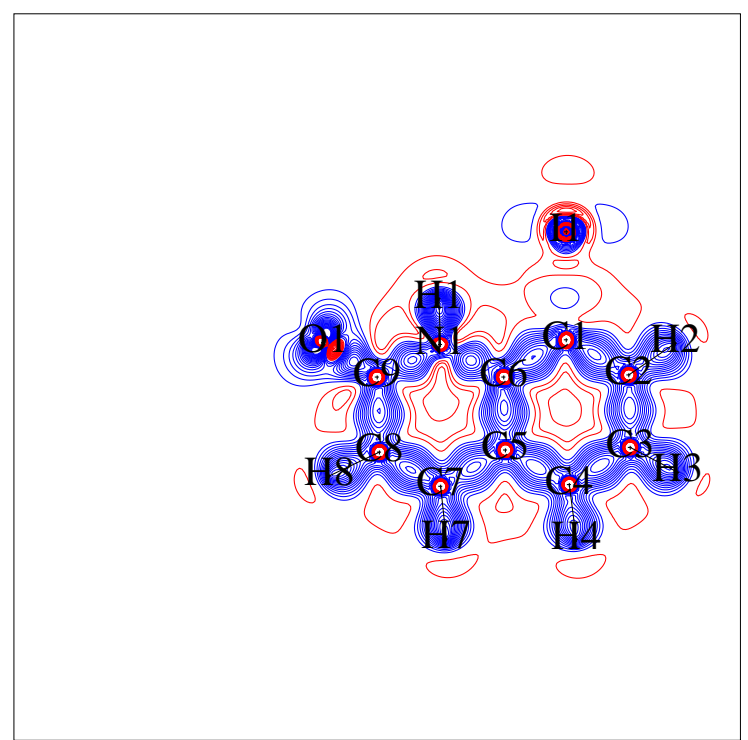

(d) HAR, anharmonic $\mathrm{n}=3$, all reflections

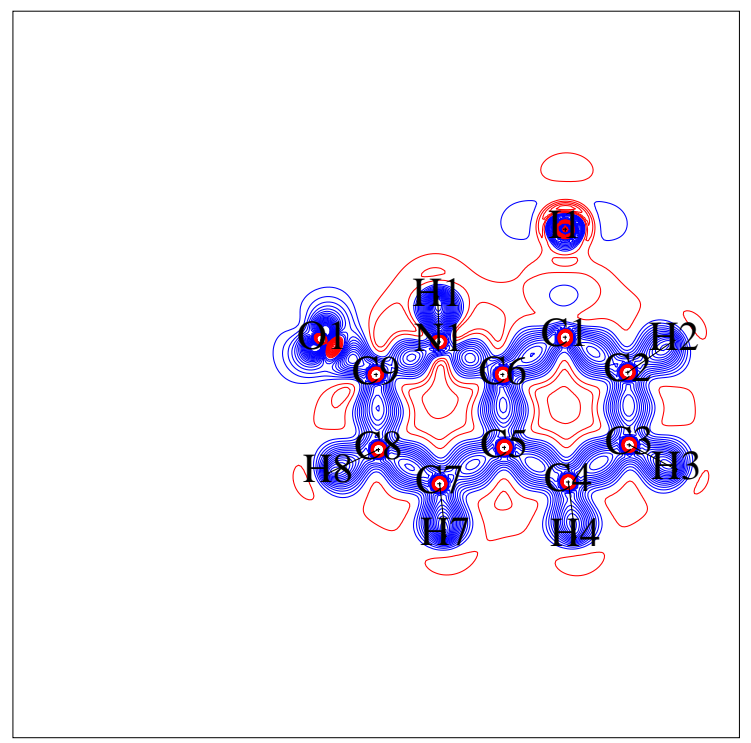

(f) HAR, anharmonic $n=4$, all reflections

Figure 48: Deformation density maps for PT-10, HAR, left: $|F| \geq 2 \sigma(|F|)$ and right: all reflections included. Contour level: $0.05 \mathrm{e} / \AA^{3}$. Colours: blue - positive, red - negative. 


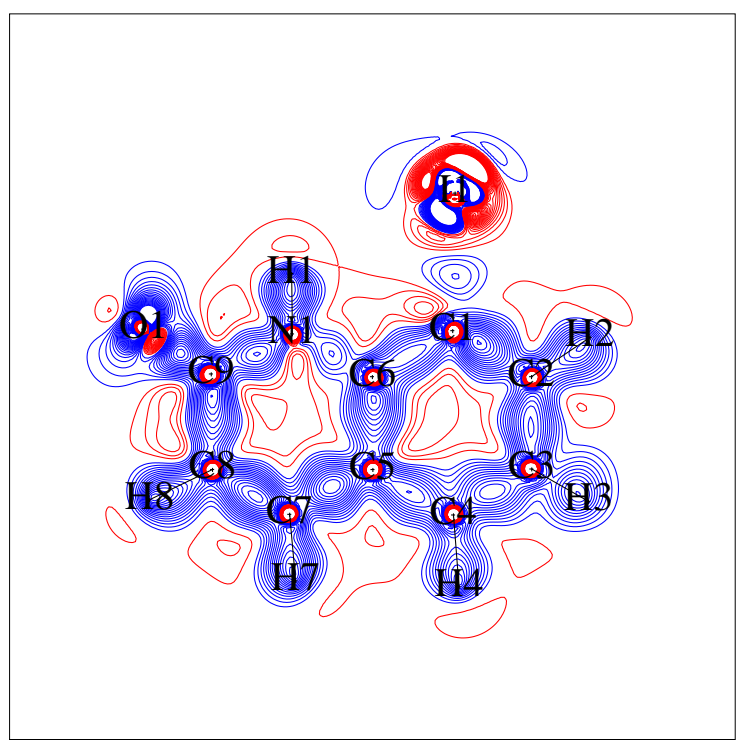

(a) XWR, harmonic, $|F| \geq 2 \sigma(|F|)$

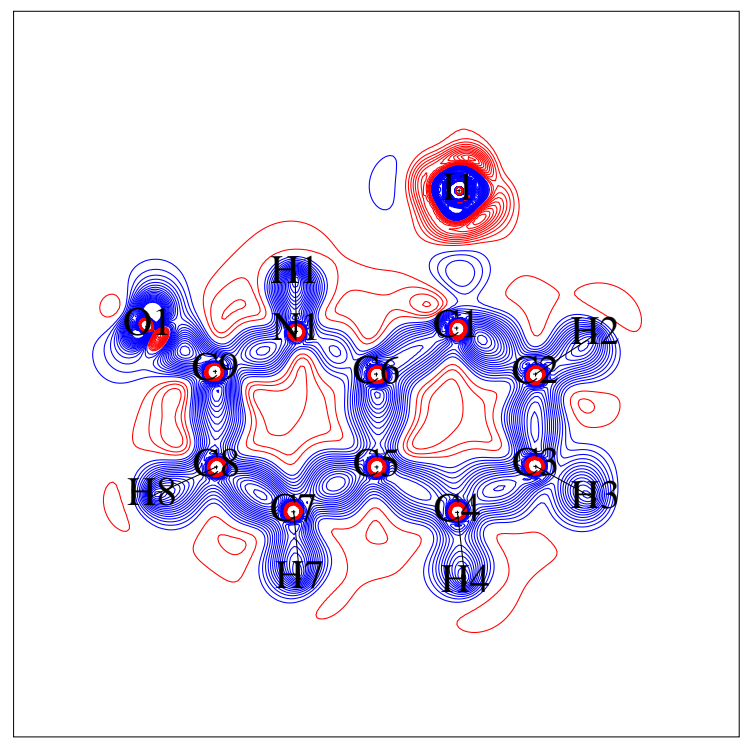

(c) XWR, anharmonic $\mathrm{n}=4,|F| \geq 2 \sigma(|F|)$

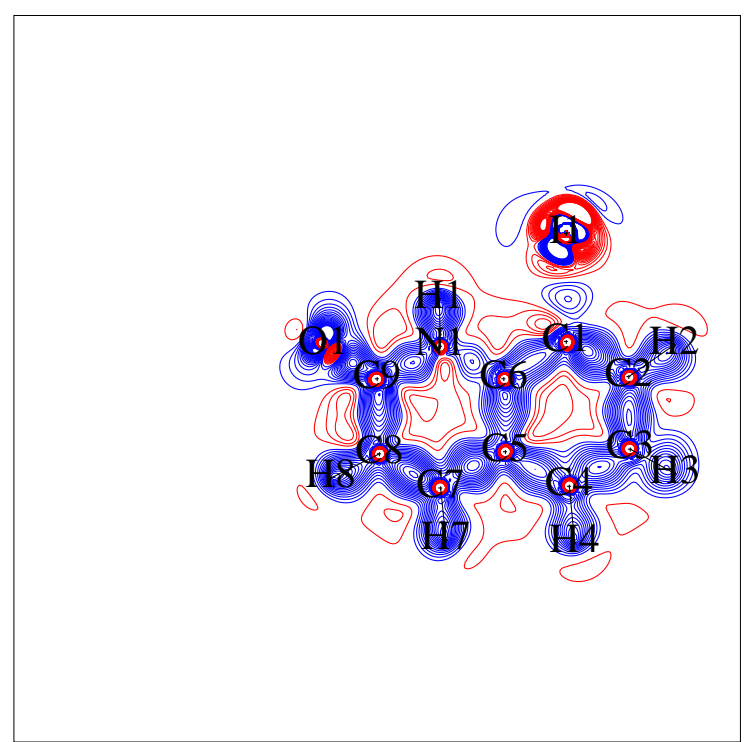

(b) XWR, harmonic, all reflections

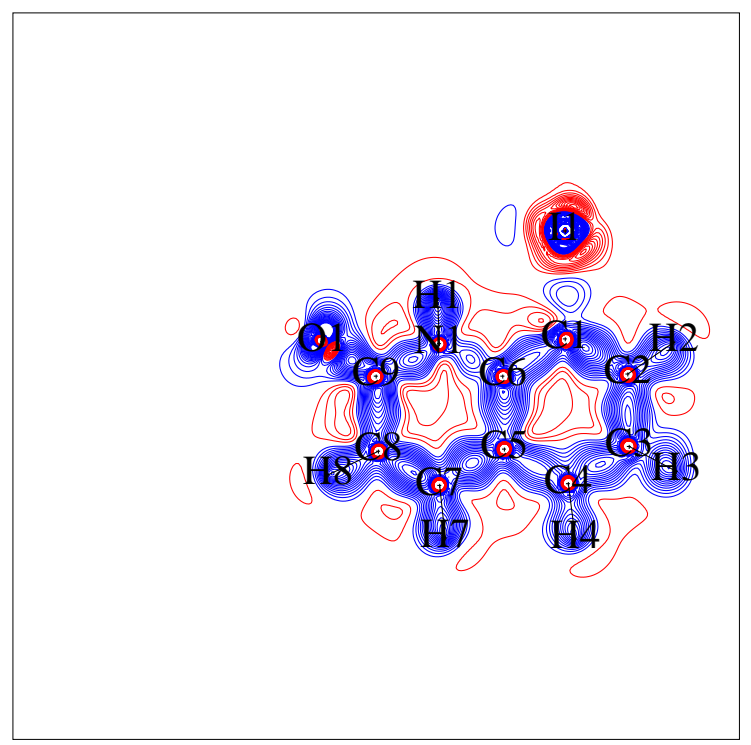

(d) XWR, anharmonic $\mathrm{n}=4$, all reflections

Figure 49: Deformation density maps for PT-10, XWR, left: $|F| \geq 2 \sigma(|F|)$ and right: all reflections included. Contour level: $0.05 \mathrm{e} / \AA^{3}$. Colours: blue - positive, red - negative. 


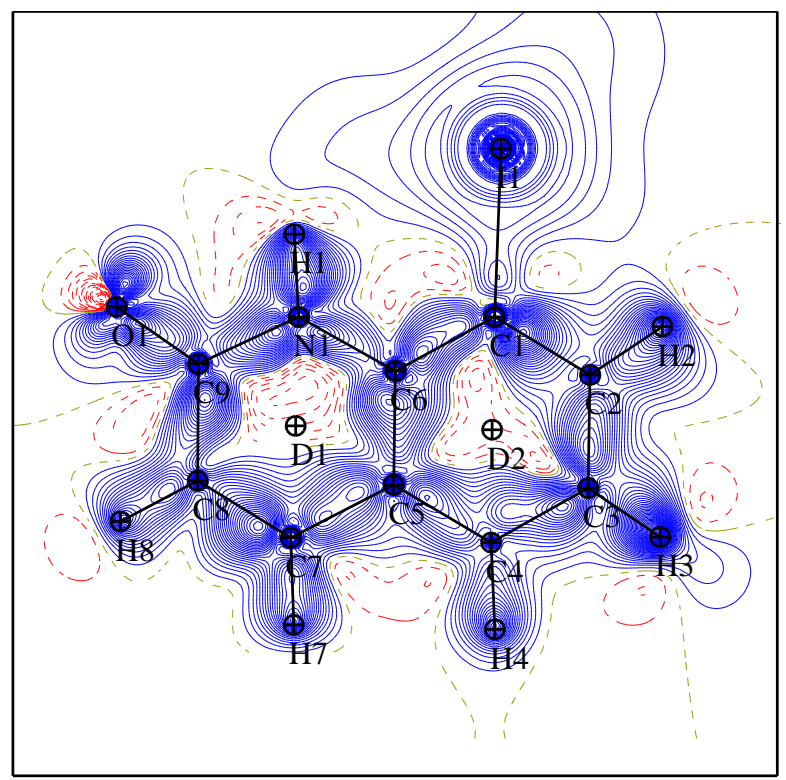

(a) MM, harmonic, $|F| \geq 2 \sigma(|F|)$

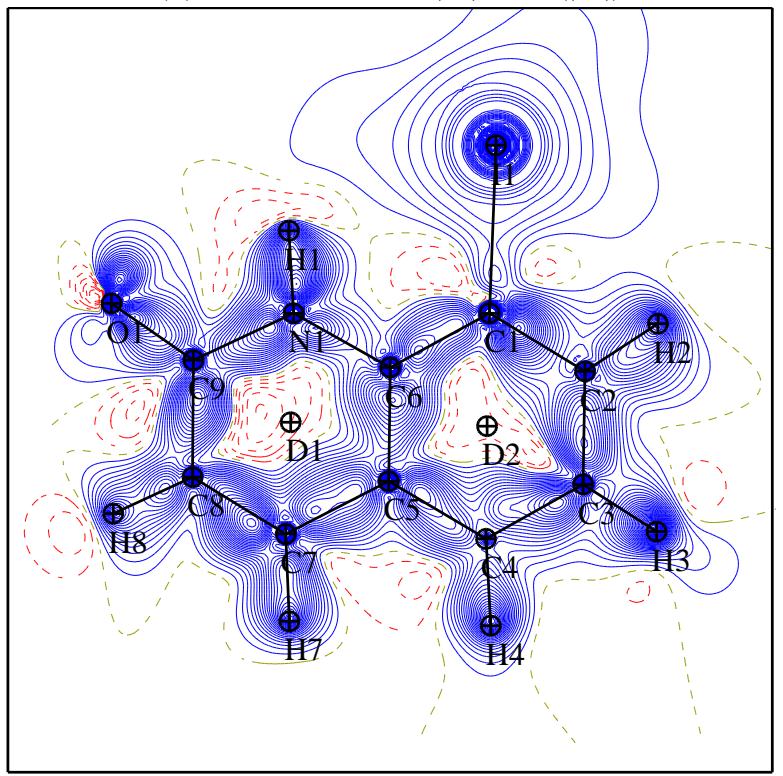

(c) MM, anharmonic n=3, $|F| \geq 2 \sigma(|F|)$

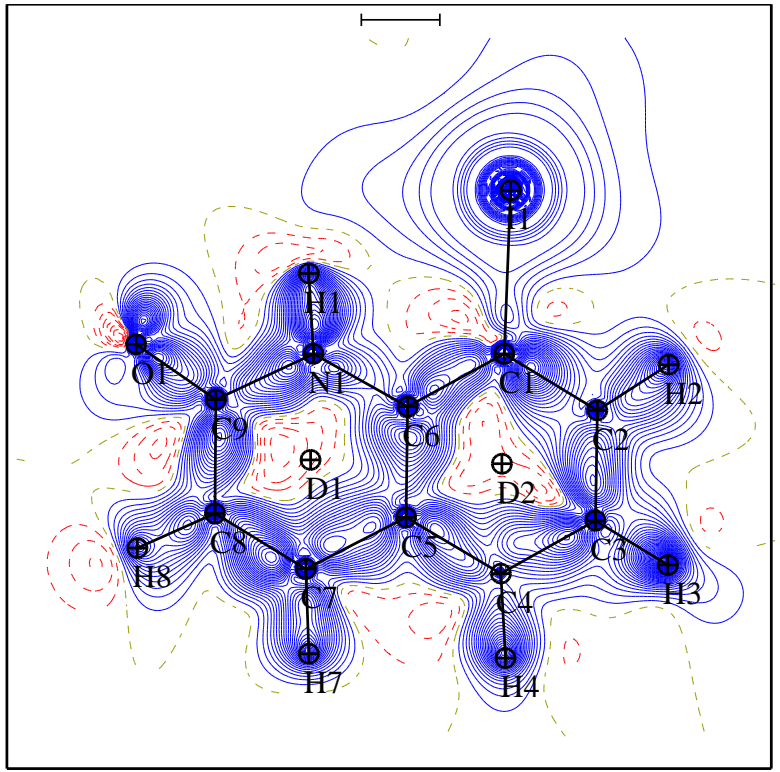

(e) MM, anharmonic n=4, $|F| \geq 2 \sigma(|F|)$

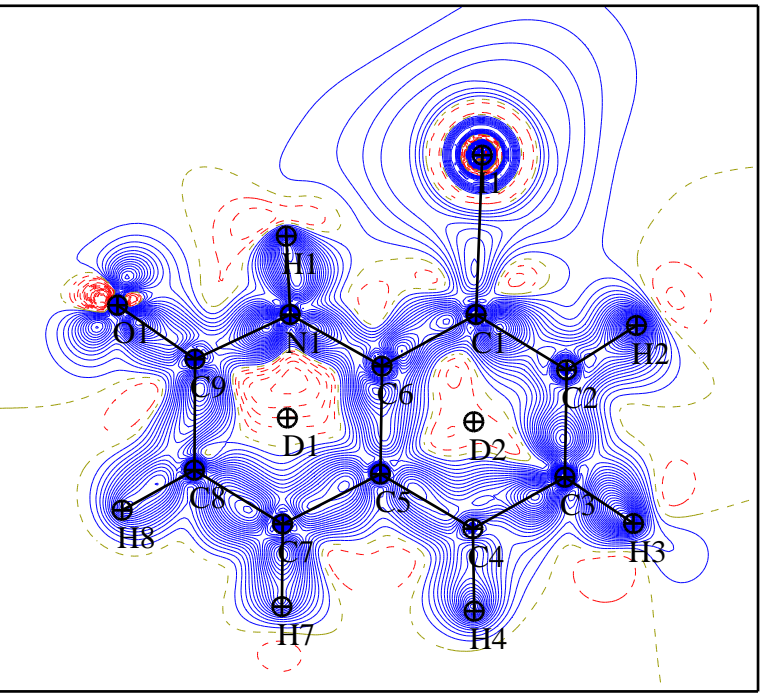

(b) MM, harmonic, all reflections

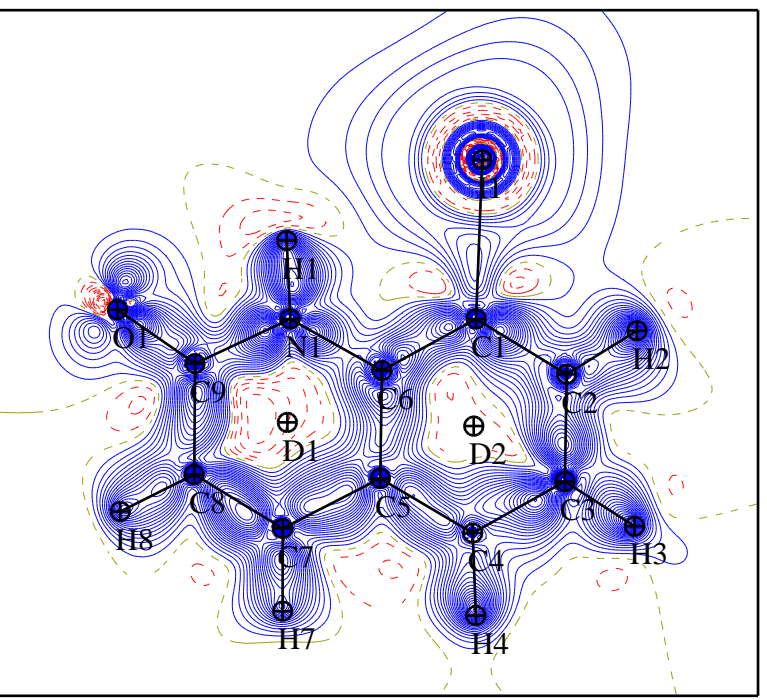

(d) MM, anharmonic $\mathrm{n}=3$, all reflections

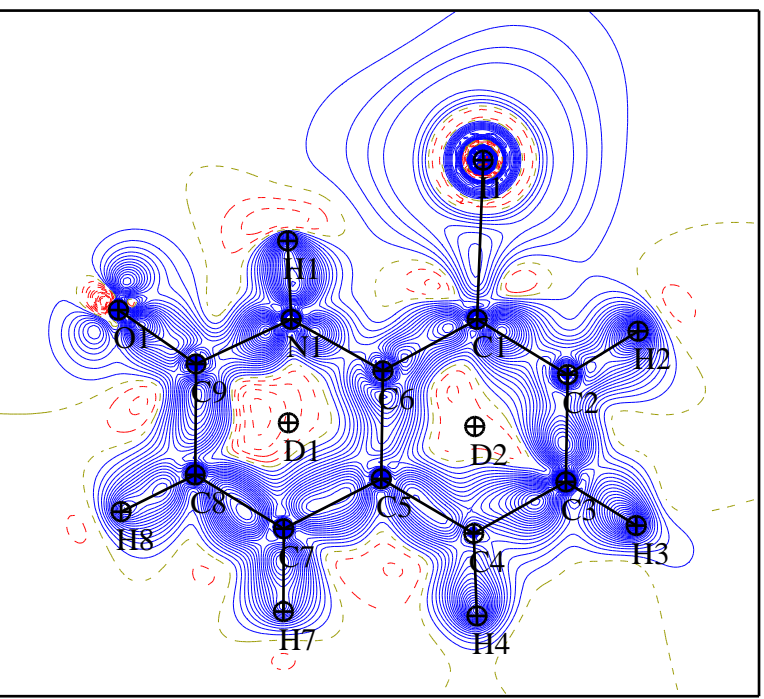

(f) MM, anharmonic $\mathrm{n}=4$, all reflections

Figure 50: Deformation density maps for PT-10, MM, restrained X-H distances, left: $|F| \geq 2 \sigma(|F|)$ and right: all reflections included. Contour level: $0.05 \mathrm{e} / \AA^{3}$. Colours: blue - positive, red - negative. 


\section{$6 \quad$ Structural motifs}

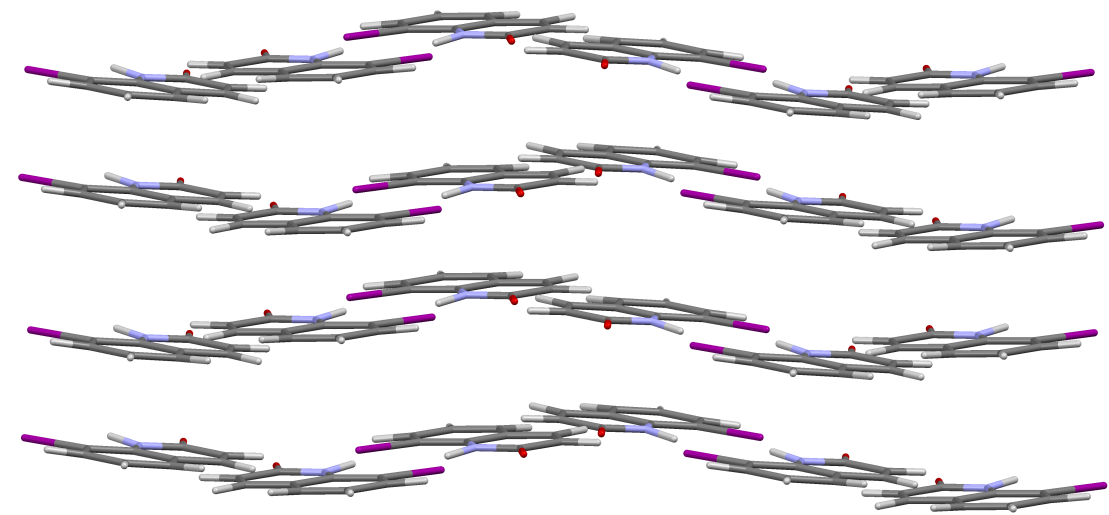

(a) Layer motifs in (102) plane.

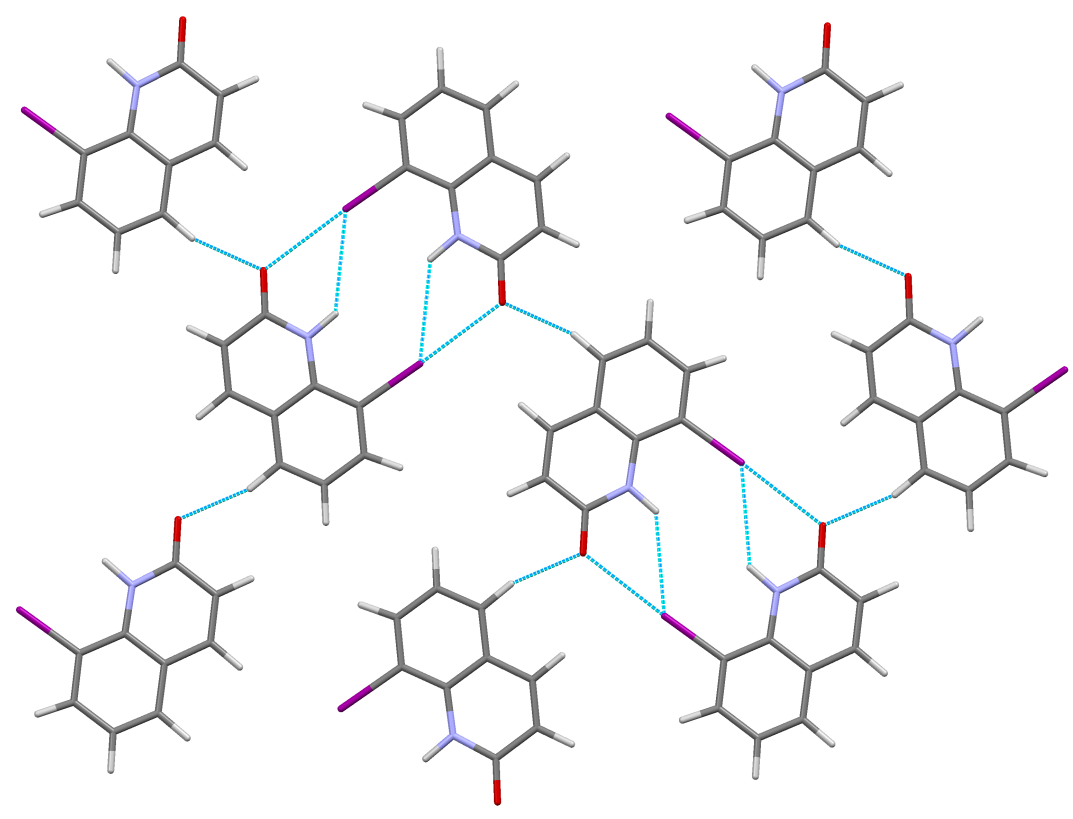

(b) Network of short contacts within a slab.

Figure 51: The layered structure of PT-10(I). 
Table 28: QTAIM parameters at bond critical points of selected interactions. Intensity cutoff: $|F| \geq 2 \sigma(|F|)$.

\begin{tabular}{|c|c|c|c|c|c|c|c|c|c|c|}
\hline structure & dimer & interaction & method & $\begin{array}{r}R \\
{[\stackrel{\circ}{A}]}\end{array}$ & $\begin{array}{r}R_{1} \\
{[\AA]} \\
{[\AA}\end{array}$ & $\begin{array}{l}R_{2} \\
{[\AA]} \\
{[\AA]}\end{array}$ & $\begin{array}{l}\rho\left(r_{C P}\right) \\
{\left[e / \AA^{3}\right]}\end{array}$ & $\begin{array}{r}\nabla^{2} \rho\left(r_{C P}\right) \\
{\left[e / \AA^{5}\right]}\end{array}$ & $\begin{array}{r}G\left(r_{C P}\right) \\
{\left[k J /\left(m o l \cdot a_{0}^{3}\right)\right]}\end{array}$ & $\left.\begin{array}{r}V\left(r_{C P}\right) \\
{\left[k J /\left(m o l \cdot a_{0}^{3}\right)\right.}\end{array}\right]$ \\
\hline \multirow[t]{6}{*}{$\mathrm{PT}-11(\mathrm{~S}-\mathrm{Ph})$} & \multirow[t]{6}{*}{ dimer1 } & \multirow[t]{6}{*}{$\mathrm{H} 1 \cdots \mathrm{O} 1$} & $\mathrm{MM}($ freeXH $)$, harm & 2.0256 & 0.8004 & 1.2254 & 0.16424 & 1.821 & 48.47 & -47.35 \\
\hline & & & $\mathrm{MM}($ freeXH $)$, anh, $\mathrm{n}=3$ & 2.0162 & 0.7899 & 1.2265 & 0.16381 & 1.781 & 47.67 & -46.85 \\
\hline & & & $\mathrm{MM}($ freeXH $)$, anh, $\mathrm{n}=3,4$ & 2.0183 & 0.7929 & 1.2257 & 0.16406 & 1.787 & 47.82 & -46.98 \\
\hline & & & MM, harm res & 1.9747 & 0.7403 & 1.2350 & 0.15993 & 1.593 & 43.66 & -43.94 \\
\hline & & & $\mathrm{MM}, \mathrm{anh}, \mathrm{n}=3$ res & 1.9753 & 0.7388 & 1.2371 & 0.15789 & 1.595 & 43.40 & -43.34 \\
\hline & & & $\mathrm{MM}$, anh, $\mathrm{n}=3,4$ res & 1.9753 & 0.7387 & 1.2372 & 0.15736 & 1.600 & 43.40 & -43.22 \\
\hline \multirow[t]{18}{*}{$\mathrm{PT}-2(\mathrm{Cl})$} & \multirow[t]{12}{*}{ dimer1 } & \multirow[t]{6}{*}{$\mathrm{Cl} 1 \cdots \mathrm{O} 1$} & MM(freeXH), harm & 3.1355 & 1.7296 & 1.4101 & 0.07043 & 0.968 & 21.33 & -16.30 \\
\hline & & & $\mathrm{MM}($ freeXH $)$, anh, $\mathrm{n}=3$ & 3.1355 & 1.7298 & 1.4100 & 0.07047 & 0.969 & 21.35 & -16.32 \\
\hline & & & $\mathrm{MM}($ freeXH $)$, anh, $\mathrm{n}=3,4$ & 3.1355 & 1.7298 & 1.4100 & 0.07047 & 0.969 & 21.35 & -16.32 \\
\hline & & & MM, harm & 3.1355 & 1.7300 & 1.4093 & 0.07037 & 0.967 & 21.32 & -16.29 \\
\hline & & & $\mathrm{MM}, \mathrm{anh}, \mathrm{n}=3$ & 3.1355 & 1.7300 & 1.4095 & 0.07036 & 0.970 & 21.36 & -16.31 \\
\hline & & & $\mathrm{MM}$, anh, $\mathrm{n}=3,4$ & 3.1355 & 1.7223 & 1.4165 & 0.06616 & 0.949 & 20.61 & -15.38 \\
\hline & & \multirow[t]{6}{*}{$\mathrm{H} 1 \cdots \mathrm{O} 1$} & $\mathrm{MM}($ freeXH $)$, harm & 1.8202 & 0.6507 & 1.1704 & 0.23539 & 1.451 & 54.42 & -69.32 \\
\hline & & & $\mathrm{MM}($ freeXH $)$, anh, $\mathrm{n}=3$ & 1.8175 & 0.6481 & 1.1703 & 0.23717 & 1.387 & 53.61 & -69.44 \\
\hline & & & $\mathrm{MM}($ freeXH), anh, $\mathrm{n}=3,4$ & 1.8175 & 0.6481 & 1.1703 & 0.23717 & 1.387 & 53.61 & -69.44 \\
\hline & & & MM, harm & 1.8422 & 0.6735 & 1.1691 & 0.23270 & 1.699 & 58.39 & -70.50 \\
\hline & & & MM, anh, n=3 & 1.8418 & 0.6740 & 1.1683 & 0.23427 & 1.663 & 58.04 & -70.80 \\
\hline & & & $\mathrm{MM}$, anh, $\mathrm{n}=3,4$ & 1.8421 & 0.6687 & 1.1738 & 0.22730 & 1.696 & 57.27 & -68.36 \\
\hline & \multirow[t]{6}{*}{ halogen } & \multirow[t]{6}{*}{$\mathrm{Cl} 1 \cdots \mathrm{C} 8$} & MM(freeXH $),$ harm & 3.4120 & 1.8138 & 1.6501 & 0.05044 & 0.525 & 11.69 & -9.07 \\
\hline & & & $\mathrm{MM}($ freeXH $)$, anh, $\mathrm{n}=3$ & 3.4123 & 1.8144 & 1.6478 & 0.05034 & 0.524 & 11.67 & -9.05 \\
\hline & & & $\mathrm{MM}($ freeXH $)$, anh, $\mathrm{n}=3,4$ & 3.4123 & 1.8144 & 1.6478 & 0.05034 & 0.524 & 11.67 & -9.06 \\
\hline & & & MM, harm & 3.4120 & 1.8143 & 1.6362 & 0.05030 & 0.533 & 11.82 & -9.13 \\
\hline & & & MM, anh, $\mathrm{n}=3$ & 3.4123 & 1.8142 & 1.6361 & 0.05021 & 0.531 & 11.78 & -9.10 \\
\hline & & & $\mathrm{MM}, \mathrm{anh}, \mathrm{n}=3,4$ & 3.4123 & 1.8052 & 1.6430 & 0.04604 & 0.511 & 11.12 & -8.34 \\
\hline \multirow[t]{9}{*}{$\mathrm{PT}-8(\mathrm{Br})$} & \multirow[t]{6}{*}{ dimer1 } & \multirow[t]{3}{*}{$\mathrm{Br} 1 \cdots \mathrm{O} 1$} & MM, harm & 3.1964 & 1.7822 & 1.4189 & 0.09298 & 1.120 & 26.30 & -22.11 \\
\hline & & & $\mathrm{MM}$, anh, $\mathrm{n}=3$ & 3.1965 & 1.7846 & 1.4169 & 0.09329 & 1.128 & 26.49 & -22.25 \\
\hline & & & $\mathrm{MM}, \mathrm{anh}, \mathrm{n}=3,4$ & 3.1964 & 1.7692 & 1.4330 & 0.08609 & 1.080 & 24.86 & -20.31 \\
\hline & & \multirow[t]{3}{*}{$\mathrm{H} 1 \cdots \mathrm{O} 1$} & MM, harm & 1.8784 & 0.7166 & 1.1623 & 0.27880 & 2.450 & 81.70 & -96.68 \\
\hline & & & $\mathrm{MM}$, anh, $\mathrm{n}=3$ & 1.8780 & 0.7195 & 1.1590 & 0.28401 & 2.411 & 82.17 & -98.66 \\
\hline & & & $\mathrm{MM}$, anh, $\mathrm{n}=3,4$ & 1.8772 & 0.7176 & 1.1600 & 0.28483 & 2.479 & 83.58 & -99.65 \\
\hline & \multirow[t]{3}{*}{ halogen } & \multirow{3}{*}{$\mathrm{Br} 1 \cdots \mathrm{H} 7$} & MM, harm & 3.0654 & 1.8280 & 1.2764 & 0.08330 & 0.800 & 19.49 & -17.20 \\
\hline & & & $\mathrm{MM}$, anh, $\mathrm{n}=3$ & 3.0623 & 1.8302 & 1.2712 & 0.08239 & 0.806 & 19.52 & -17.08 \\
\hline & & & $\mathrm{MM}, \mathrm{anh}, \mathrm{n}=3,4$ & 3.0611 & 1.8134 & 1.2899 & 0.07620 & 0.781 & 18.46 & -15.65 \\
\hline PT-10(I) & halogen & $\mathrm{I} 1 \cdots \mathrm{O} 1$ & MM, harm & 2.9167 & 1.6834 & 1.2438 & 0.24661 & 1.705 & 61.30 & -76.16 \\
\hline & & & MM, anh, n=3 & 2.9215 & 1.6822 & 1.2477 & 0.24348 & 1.737 & 61.24 & -75.17 \\
\hline & & & MM, anh, $\mathrm{n}=3,4$ & 2.9217 & 1.6794 & 1.2459 & 0.24788 & 1.764 & 62.63 & -77.21 \\
\hline & & I $1 \cdots \mathrm{H} 1$ & MM, harm & 2.9691 & 2.1952 & 1.3561 & 0.15512 & 0.011 & 14.21 & -28.12 \\
\hline & & & $\mathrm{MM}, \mathrm{anh}, \mathrm{n}=3$ & 2.9677 & 2.1237 & 1.2149 & 0.13742 & 0.062 & 12.58 & -23.46 \\
\hline & & & $\mathrm{MM}, \mathrm{anh}, \mathrm{n}=3,4$ & 2.9665 & 2.2478 & 1.3409 & 0.13833 & -0.009 & 11.42 & -23.07 \\
\hline & dimer1 & $\mathrm{C} 3 \cdots \mathrm{C} 6$ & MM, harm & 3.3318 & 1.7156 & 1.7170 & 0.03239 & 0.478 & 9.70 & -6.39 \\
\hline & & & $\mathrm{MM}, \mathrm{anh}, \mathrm{n}=3$ & 3.3314 & 1.7332 & 1.7344 & 0.03510 & 0.462 & 9.57 & -6.55 \\
\hline & & & $\mathrm{MM}$, anh, $\mathrm{n}=3,4$ & 3.3315 & 1.7355 & 1.7474 & 0.03605 & 0.454 & 9.48 & -6.59 \\
\hline & & $\mathrm{C} 3 \cdots \mathrm{N} 1$ & MM, harm & 3.6205 & 1.8064 & 1.8649 & 0.04201 & 0.478 & 10.26 & -7.51 \\
\hline & & & $\mathrm{MM}$, anh, $\mathrm{n}=3$ & 3.6204 & 1.8041 & 1.8638 & 0.04242 & 0.473 & 10.21 & -7.53 \\
\hline & & & $\mathrm{MM}, \mathrm{anh}, \mathrm{n}=3,4$ & 3.6205 & 1.8090 & 1.8591 & 0.04276 & 0.475 & 10.25 & -7.58 \\
\hline & & $\mathrm{C} 4 \cdots \mathrm{C} 7$ & $\mathrm{MM}$, harm & 3.6017 & 1.7391 & 1.8696 & 0.03444 & 0.404 & 8.47 & -5.95 \\
\hline & & & $\mathrm{MM}, \mathrm{anh}, \mathrm{n}=3$ & 3.6015 & 1.7525 & 1.8564 & 0.03650 & 0.403 & 8.57 & -6.17 \\
\hline & & & $\mathrm{MM}$, anh, $\mathrm{n}=3,4$ & 3.6016 & 1.7453 & 1.8625 & 0.03548 & 0.403 & 8.51 & -6.05 \\
\hline & & $\mathrm{N} 1 \cdots \mathrm{C} 5$ & MM, harm & 3.5729 & 1.8295 & 1.7441 & 0.03934 & 0.469 & 9.93 & -7.10 \\
\hline & & & $\mathrm{MM}$, anh, $\mathrm{n}=3$ & 3.5732 & 1.8362 & 1.7387 & 0.04117 & 0.475 & 10.17 & -7.39 \\
\hline & & & MM, anh, n=3,4 & 3.5732 & 1.8244 & 1.7492 & 0.04214 & 0.473 & 10.18 & -7.48 \\
\hline & & $\mathrm{C} 7 \cdots \mathrm{O} 1$ & MM, harm & 3.6387 & 1.8526 & 1.8099 & 0.03964 & 0.361 & 7.99 & -6.16 \\
\hline & & & MM, anh, $\mathrm{n}=3$ & 3.6389 & 1.8566 & 1.8085 & 0.04006 & 0.372 & 8.22 & -6.31 \\
\hline & & & $\mathrm{MM}, \mathrm{anh}, \mathrm{n}=3,4$ & 3.6390 & 1.8546 & 1.8166 & 0.04015 & 0.368 & 8.16 & -6.29 \\
\hline & & $\mathrm{C} 7 \cdots \mathrm{C} 8$ & MM, harm & 3.6100 & 1.8829 & 1.8197 & 0.02878 & 0.292 & 6.14 & -4.34 \\
\hline & & & $\mathrm{MM}, \mathrm{anh}, \mathrm{n}=3$ & 3.6089 & 1.8862 & 1.8401 & 0.03329 & 0.298 & 6.49 & -4.86 \\
\hline & & & $\mathrm{MM}$, anh, $\mathrm{n}=3,4$ & 3.6088 & 1.8681 & 1.8228 & 0.03328 & 0.302 & 6.55 & -4.89 \\
\hline
\end{tabular}


Table 29: QTAIM parameters at bond critical points of selected interactions. No intensity cutoff. In the case of PT-10(I) calculations of QTAIM properties for certain critical points failed.

\begin{tabular}{|c|c|c|c|c|c|c|c|c|c|c|}
\hline structure & dimer & interaction & method & $\begin{array}{r}R \\
{[\AA]}\end{array}$ & $\begin{array}{r}R_{1} \\
{[\AA]} \\
{[\AA]}\end{array}$ & $\begin{array}{l}R_{2} \\
{[\AA]} \\
{[\AA]}\end{array}$ & $\begin{array}{l}\rho\left(r_{C P}\right) \\
{\left[e / \AA^{3}\right]}\end{array}$ & $\begin{array}{r}\nabla^{2} \rho\left(r_{C P}\right) \\
{\left[e / \AA^{5}\right]}\end{array}$ & $\begin{array}{r}G\left(r_{C P}\right) \\
{\left[k J /\left(m o l \cdot a_{0}^{3}\right)\right]}\end{array}$ & $\begin{array}{r}V\left(r_{C P}\right) \\
{\left[k J /\left(m o l \cdot a_{0}^{3}\right)\right]}\end{array}$ \\
\hline \multirow[t]{6}{*}{ PT-11(S-Ph) } & \multirow[t]{6}{*}{ dimer1 } & \multirow[t]{6}{*}{$\mathrm{H} 1 \cdots \mathrm{O} 1$} & $\mathrm{MM}($ freeXH $)$, harm & 2.0104 & 0.7772 & 1.2336 & 0.1612 & 1.716 & 46.09 & -45.45 \\
\hline & & & $\mathrm{MM}($ freeXH $)$, anh, $\mathrm{n}=3$ & 2.0046 & 0.7702 & 1.2349 & 0.1603 & 1.688 & 45.44 & -44.92 \\
\hline & & & $\mathrm{MM}($ freeXH), anh, $\mathrm{n}=3,4$ & 2.0061 & 0.7724 & 1.2342 & 0.1606 & 1.696 & 45.63 & -45.07 \\
\hline & & & MM, harm res & 1.9750 & 0.7338 & 1.2420 & 0.1579 & 1.539 & 42.37 & -42.83 \\
\hline & & & $\mathrm{MM}, \mathrm{anh}, \mathrm{n}=3$ res & 1.9756 & 0.7353 & 1.2412 & 0.1579 & 1.532 & 42.24 & -42.76 \\
\hline & & & MM, anh, $\mathrm{n}=3,4$ res & 1.9755 & 0.7343 & 1.2421 & 0.1569 & 1.536 & 42.16 & -42.49 \\
\hline \multirow[t]{18}{*}{$\mathrm{PT}-2(\mathrm{Cl})$} & \multirow[t]{12}{*}{ dimer1 } & \multirow[t]{6}{*}{$\mathrm{Cl} 1 \cdots \mathrm{O} 1$} & $\mathrm{MM}($ freeXH) harm & 3.1356 & 1.7387 & 1.4012 & 0.0708 & 0.953 & 21.09 & -16.23 \\
\hline & & & $\mathrm{MM}($ freeXH $)$ anh, $\mathrm{n}=3$ & 3.1356 & 1.7383 & 1.4017 & 0.0706 & 0.953 & 21.08 & -16.19 \\
\hline & & & $\mathrm{MM}($ freeXH) anh, $\mathrm{n}=3,4$ & 3.1356 & 1.7303 & 1.4093 & 0.0665 & 0.933 & 20.35 & -15.29 \\
\hline & & & MM, harm & 3.1356 & 1.7387 & 1.4011 & 0.0708 & 0.953 & 21.10 & -16.23 \\
\hline & & & MM, anh, $\mathrm{n}=3$ & 3.1356 & 1.7383 & 1.4017 & 0.0706 & 0.953 & 21.08 & -16.19 \\
\hline & & & $\mathrm{MM}, \mathrm{anh}, \mathrm{n}=3,4$ & 3.1356 & 1.7303 & 1.4093 & 0.0665 & 0.933 & 20.35 & -15.29 \\
\hline & & \multirow[t]{6}{*}{$\mathrm{H} 1 \cdots \mathrm{O} 1$} & MM(freeXH) harm & 1.8339 & 0.6538 & 1.1804 & 0.2130 & 1.774 & 55.98 & -63.64 \\
\hline & & & $\mathrm{MM}($ freeXH $)$ anh, $\mathrm{n}=3$ & 1.8325 & 0.6525 & 1.1804 & 0.2140 & 1.730 & 55.36 & -63.62 \\
\hline & & & $\mathrm{MM}($ freeXH) anh, $\mathrm{n}=3,4$ & 1.8356 & 0.6497 & 1.1861 & 0.2073 & 1.790 & 55.22 & -61.68 \\
\hline & & & MM, harm & 1.8339 & 0.6539 & 1.1804 & 0.2130 & 1.774 & 55.97 & -63.63 \\
\hline & & & MM, anh, n=3 & 1.8325 & 0.6525 & 1.1804 & 0.2140 & 1.730 & 55.36 & -63.62 \\
\hline & & & $\mathrm{MM}$, anh, $\mathrm{n}=3,4$ & 1.8356 & 0.6497 & 1.1861 & 0.2073 & 1.790 & 55.22 & -61.68 \\
\hline & \multirow[t]{6}{*}{ halogen } & \multirow[t]{6}{*}{$\mathrm{Cl} 1 \cdots \mathrm{C} 8$} & MM(freeXH) harm & 3.4121 & 1.8076 & 1.6653 & 0.0510 & 0.519 & 11.62 & -9.10 \\
\hline & & & $\mathrm{MM}($ freeXH) anh, $\mathrm{n}=3$ & 3.4123 & 1.8073 & 1.6628 & 0.0511 & 0.519 & 11.62 & -9.11 \\
\hline & & & $\mathrm{MM}($ freeXH $)$ anh, $\mathrm{n}=3,4$ & 3.4124 & 1.7983 & 1.6698 & 0.0467 & 0.500 & 10.97 & -8.33 \\
\hline & & & MM, harm & 3.4121 & 1.8076 & 1.6654 & 0.0510 & 0.519 & 11.62 & -9.10 \\
\hline & & & $\mathrm{MM}, \mathrm{anh}, \mathrm{n}=3$ & 3.4123 & 1.8073 & 1.6628 & 0.0511 & 0.519 & 11.62 & -9.11 \\
\hline & & & $\mathrm{MM}, \mathrm{anh}, \mathrm{n}=3,4$ & 3.4124 & 1.7983 & 1.6698 & 0.0467 & 0.500 & 10.97 & -8.33 \\
\hline \multirow{9}{*}{ PT-8(Br) } & \multirow[t]{6}{*}{ dimer1 } & \multirow[t]{3}{*}{$\mathrm{Br} 1 \cdots \mathrm{O} 1$} & MM harm & 3.1966 & 1.7868 & 1.4150 & 0.0923 & 1.123 & 26.28 & -21.98 \\
\hline & & & MM, anh, $\mathrm{n}=3$ & 3.1966 & 1.7887 & 1.4134 & 0.0926 & 1.130 & 26.45 & -22.11 \\
\hline & & & MM, anh, $\mathrm{n}=3,4$ & 3.1966 & 1.7632 & 1.4400 & 0.0809 & 1.052 & 23.84 & -19.03 \\
\hline & & \multirow{3}{*}{$\mathrm{H} 1 \cdots \mathrm{O} 1$} & MM, harm & 1.8750 & 0.7157 & 1.1597 & 0.2780 & 2.376 & 80.19 & -95.67 \\
\hline & & & $\mathrm{MM}, \mathrm{anh}, \mathrm{n}=3$ & 1.8746 & 0.7181 & 1.1570 & 0.2823 & 2.356 & 80.79 & -97.41 \\
\hline & & & $\mathrm{MM}, \mathrm{anh}, \mathrm{n}=3,4$ & 1.8735 & 0.7162 & 1.1577 & 0.2855 & 2.438 & 82.98 & -99.56 \\
\hline & \multirow[t]{3}{*}{ halogen } & \multirow[t]{3}{*}{$\mathrm{Br} 1 \cdots \mathrm{H} 7$} & MM, harm & 3.0643 & 1.8311 & 1.2591 & 0.0830 & 0.798 & 19.43 & -17.13 \\
\hline & & & $\mathrm{MM}$, anh, $\mathrm{n}=3$ & 3.0610 & 1.8334 & 1.2532 & 0.0820 & 0.803 & 19.42 & -16.97 \\
\hline & & & MM, anh, $\mathrm{n}=3,4$ & 3.0604 & 1.8077 & 1.2784 & 0.0701 & 0.749 & 17.34 & -14.26 \\
\hline PT-10(I) & halogen & $\mathrm{I} 1 \cdots \mathrm{O} 1$ & MM harm & 2.9168 & 1.6929 & 1.2259 & 0.2653 & 2.024 & 71.04 & -86.93 \\
\hline & & & MM, anh, $\mathrm{n}=3$ & 2.9212 & 1.6998 & 1.2233 & 0.2768 & 1.964 & 72.45 & -91.40 \\
\hline & & & $\mathrm{MM}, \mathrm{anh}, \mathrm{n}=3,4$ & 2.9213 & 1.6949 & 1.2274 & 0.2672 & 1.980 & 70.64 & -87.33 \\
\hline & & $\mathrm{I} 1 \cdots \mathrm{H} 1$ & MM, harm & 2.9682 & 2.0278 & 1.0935 & 0.1402 & 0.263 & 16.61 & -26.05 \\
\hline & & & MM, anh, n=3 & 2.9747 & 2.0646 & 1.0182 & 0.1325 & 0.252 & 15.35 & -23.84 \\
\hline & & & MM, anh, $\mathrm{n}=3,4$ & 2.9740 & 2.0796 & 1.0369 & 0.1274 & 0.236 & 14.37 & -22.31 \\
\hline & dimer1 & $\mathrm{C} 3 \cdots \mathrm{C} 6$ & MM, harm & $\mathrm{N} / \mathrm{A}$ & $\mathrm{N} / \mathrm{A}$ & $\mathrm{N} / \mathrm{A}$ & $\mathrm{N} / \mathrm{A}$ & $\mathrm{N} / \mathrm{A}$ & $\mathrm{N} / \mathrm{A}$ & $\mathrm{N} / \mathrm{A}$ \\
\hline & & & MM, anh, $\mathrm{n}=3$ & 3.3309 & 1.7436 & 1.6890 & 0.0384 & 0.485 & 10.16 & -7.13 \\
\hline & & & MM, anh, $\mathrm{n}=3,4$ & 3.3309 & 1.7387 & 1.6903 & 0.0386 & 0.488 & 10.24 & -7.19 \\
\hline & & $\mathrm{C} 3 \cdots \mathrm{N} 1$ & MM, harm & $\mathrm{N} / \mathrm{A}$ & $\mathrm{N} / \mathrm{A}$ & $\mathrm{N} / \mathrm{A}$ & $\mathrm{N} / \mathrm{A}$ & $\mathrm{N} / \mathrm{A}$ & $\mathrm{N} / \mathrm{A}$ & $\mathrm{N} / \mathrm{A}$ \\
\hline & & & $\mathrm{MM}, \mathrm{anh}, \mathrm{n}=3$ & 3.6212 & 1.8986 & 1.8213 & 0.0430 & 0.484 & 10.43 & -7.70 \\
\hline & & & MM, anh, $\mathrm{n}=3,4$ & $\mathrm{~N} / \mathrm{A}$ & $\mathrm{N} / \mathrm{A}$ & $\mathrm{N} / \mathrm{A}$ & $\mathrm{N} / \mathrm{A}$ & $\mathrm{N} / \mathrm{A}$ & $\mathrm{N} / \mathrm{A}$ & $\mathrm{N} / \mathrm{A}$ \\
\hline & & $\mathrm{C} 4 \cdots \mathrm{C} 7$ & MM, harm & N/A & N/A & N/A & N/A & $\mathrm{N} / \mathrm{A}$ & $\mathrm{N} / \mathrm{A}$ & $\mathrm{N} / \mathrm{A}$ \\
\hline & & & MM, anh, $\mathrm{n}=3$ & 3.6013 & 1.7553 & 1.8867 & 0.0393 & 0.399 & 8.66 & -6.46 \\
\hline & & & $\mathrm{MM}^{\prime}$, anh, $\mathrm{n}=3,4$ & 3.6013 & 1.7599 & 1.8796 & 0.0411 & 0.404 & 8.87 & -6.73 \\
\hline & & $\mathrm{N} 1 \cdots \mathrm{C} 5$ & MM, harm & $\mathrm{N} / \mathrm{A}$ & $\mathrm{N} / \mathrm{A}$ & $\mathrm{N} / \mathrm{A}$ & N/A & $\mathrm{N} / \mathrm{A}$ & $\mathrm{N} / \mathrm{A}$ & $\mathrm{N} / \mathrm{A}$ \\
\hline & & & MM, anh, $\mathrm{n}=3$ & $\mathrm{~N} / \mathrm{A}$ & $\mathrm{N} / \mathrm{A}$ & $\mathrm{N} / \mathrm{A}$ & $\mathrm{N} / \mathrm{A}$ & $\mathrm{N} / \mathrm{A}$ & N/A & N/A \\
\hline & & & MM, anh, $\mathrm{n}=3,4$ & $\mathrm{~N} / \mathrm{A}$ & $\mathrm{N} / \mathrm{A}$ & $\mathrm{N} / \mathrm{A}$ & $\mathrm{N} / \mathrm{A}$ & $\mathrm{N} / \mathrm{A}$ & $\mathrm{N} / \mathrm{A}$ & $\mathrm{N} / \mathrm{A}$ \\
\hline & & $\mathrm{C} 7 \cdots \mathrm{O} 1$ & MM, harm & 3.6381 & 1.8187 & 1.8313 & 0.0397 & 0.354 & 7.88 & -6.10 \\
\hline & & & MM, anh, n=3 & 3.6382 & 1.8184 & 1.8358 & 0.0386 & 0.350 & 7.73 & -5.94 \\
\hline & & & MM, anh, $\mathrm{n}=3,4$ & 3.6383 & 1.8231 & 1.8355 & 0.0393 & 0.352 & 7.81 & -6.04 \\
\hline & & $\mathrm{C} 7 \cdots \mathrm{C} 8$ & MM, harm & $\mathrm{N} / \mathrm{A}$ & $\mathrm{N} / \mathrm{A}$ & $\mathrm{N} / \mathrm{A}$ & $\mathrm{N} / \mathrm{A}$ & $\mathrm{N} / \mathrm{A}$ & $\mathrm{N} / \mathrm{A}$ & $\mathrm{N} / \mathrm{A}$ \\
\hline & & & MM, anh, $\mathrm{n}=3$ & $\mathrm{~N} / \mathrm{A}$ & $\mathrm{N} / \mathrm{A}$ & $\mathrm{N} / \mathrm{A}$ & $\mathrm{N} / \mathrm{A}$ & $\mathrm{N} / \mathrm{A}$ & $\mathrm{N} / \mathrm{A}$ & $\mathrm{N} / \mathrm{A}$ \\
\hline & & & $\mathrm{MM}, \mathrm{anh}, \mathrm{n}=3,4$ & $\mathrm{~N} / \mathrm{A}$ & N/A & $\mathrm{N} / \mathrm{A}$ & N/A & $\mathrm{N} / \mathrm{A}$ & N/A & N/A \\
\hline
\end{tabular}




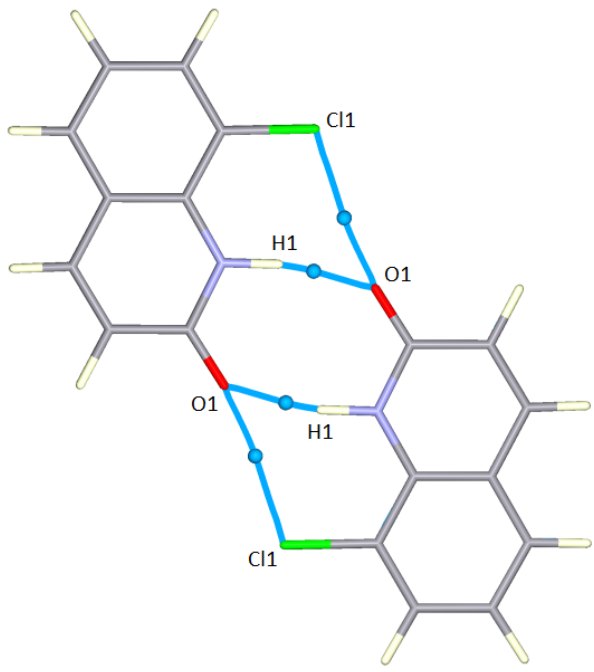

(a) PT-2(Cl), dimer1

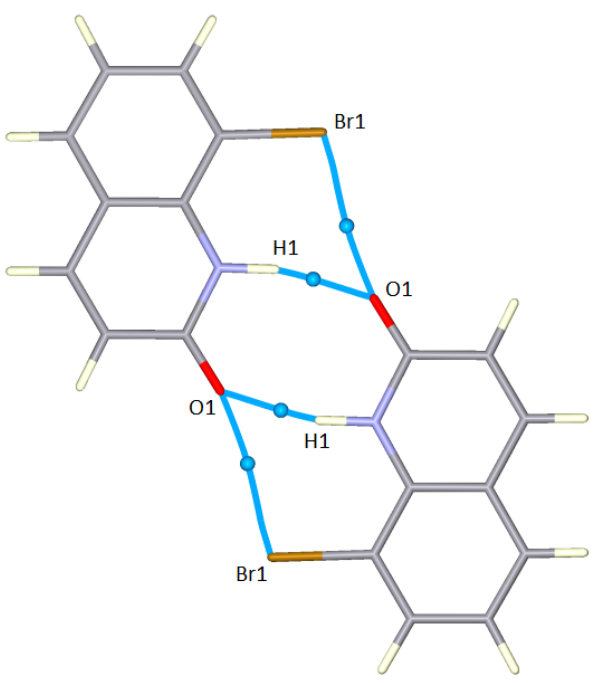

(c) PT-8(Br), dimer1

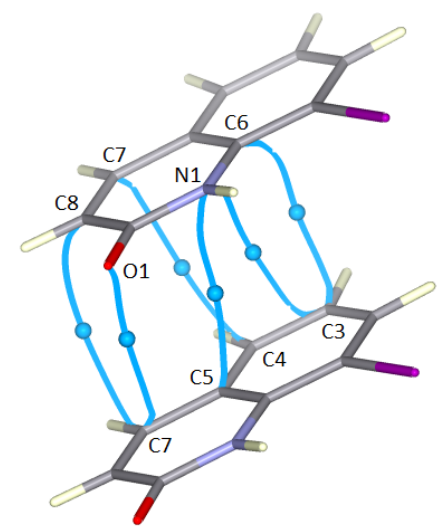

(e) PT-10(I), dimer1

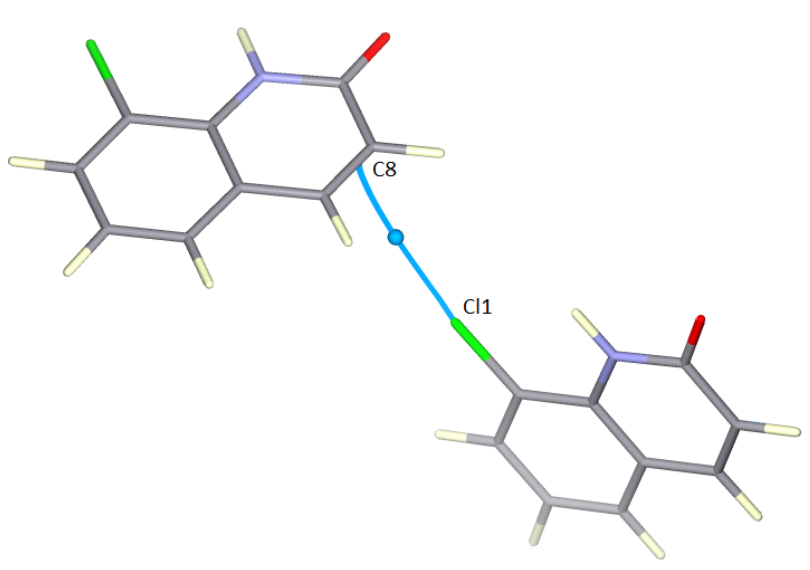

(b) PT-2(Cl), halogen

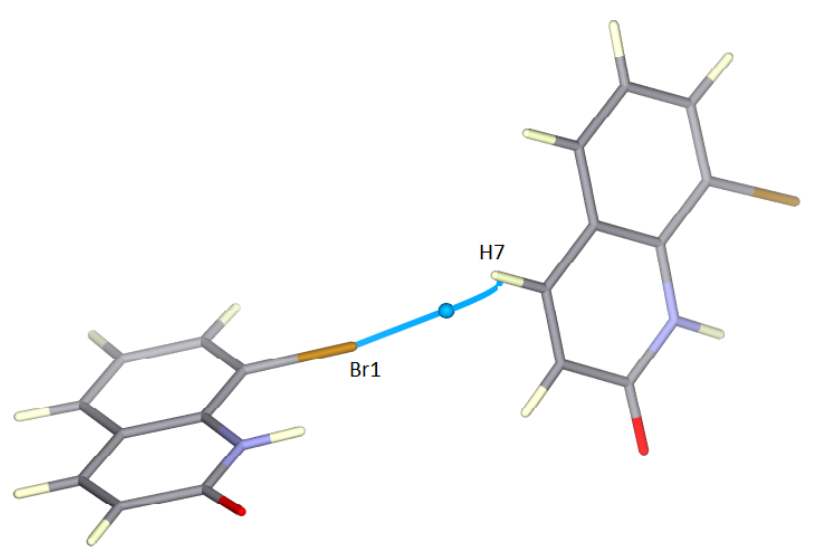

(d) PT-8(Br), halogen

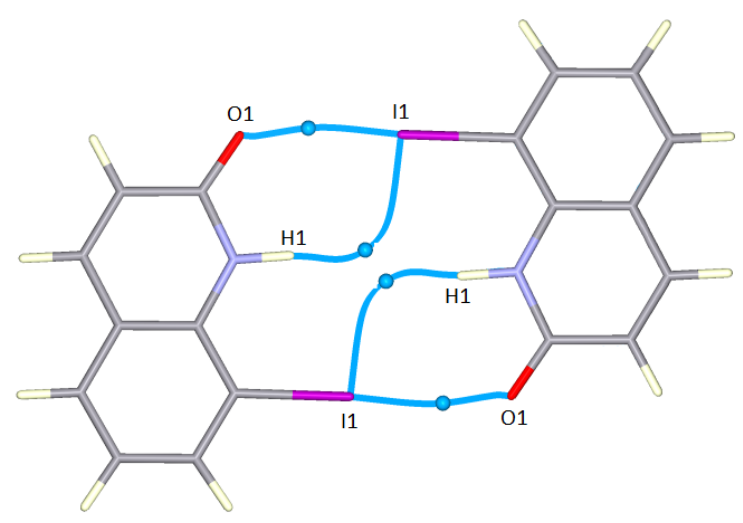

(f) PT-10(I), halogen

Figure 52: Bond critical points found for hydrogen, halogen and stacking interactions. 


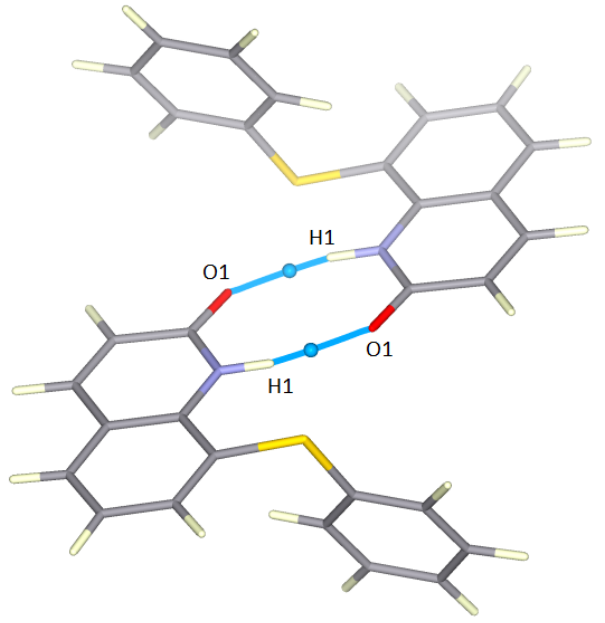

(a) PT-11(S-Ph), dimer1

Figure 53: Bond critical points found for hydrogen, halogen and stacking interactions. 
7 Dimer interaction energy as a function of centroid separation of dimers

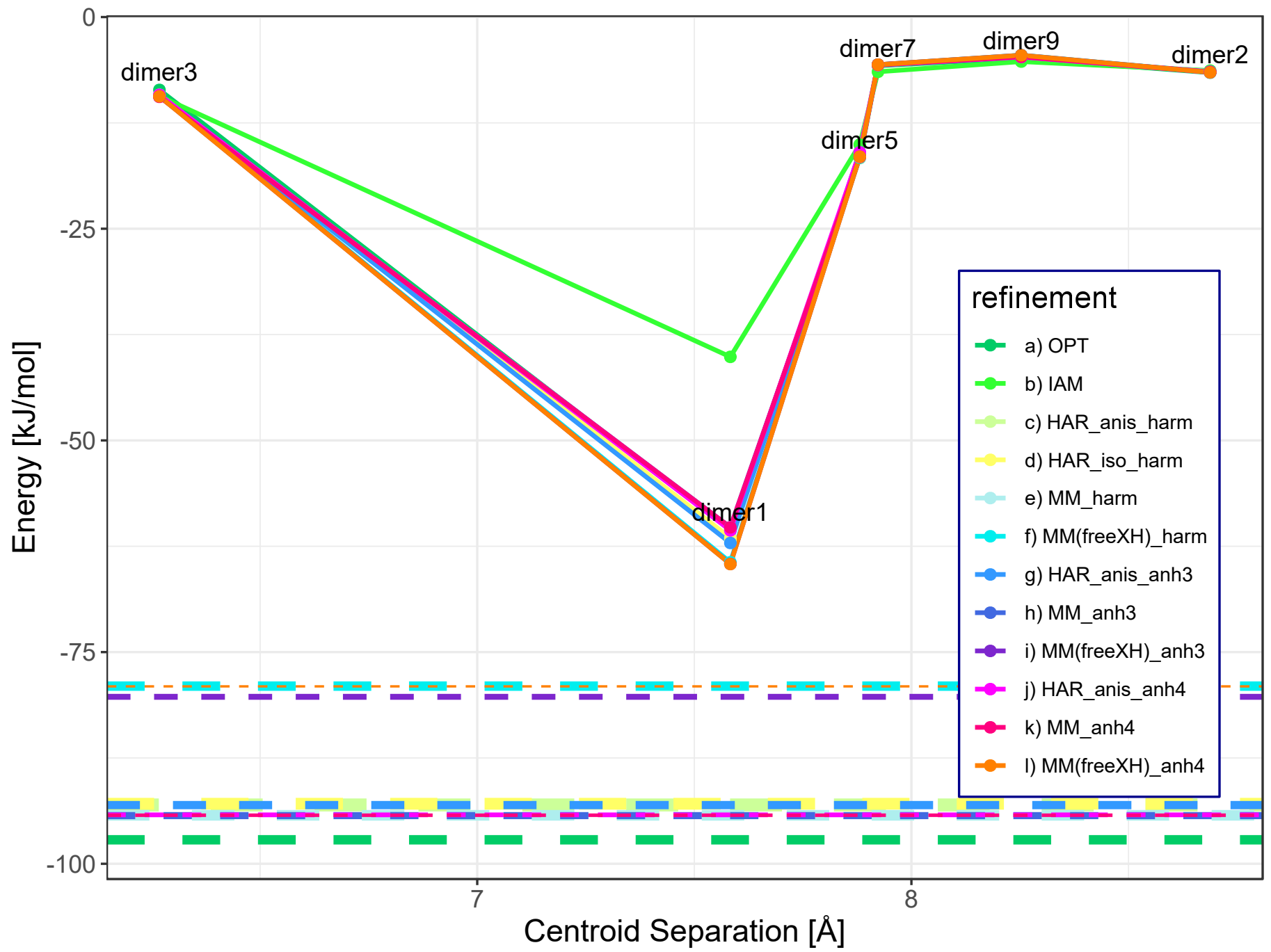

Figure 54: Dimer interaction energies (data points linked with solid lines) obtained for the structure of PT-2(Cl) refined with intensity cut-off $(|F| \geq 2 \sigma(|F|))$ using various methods presented as a function of centroid separation of dimers. Cohesive energies are marked with dashed lines. 


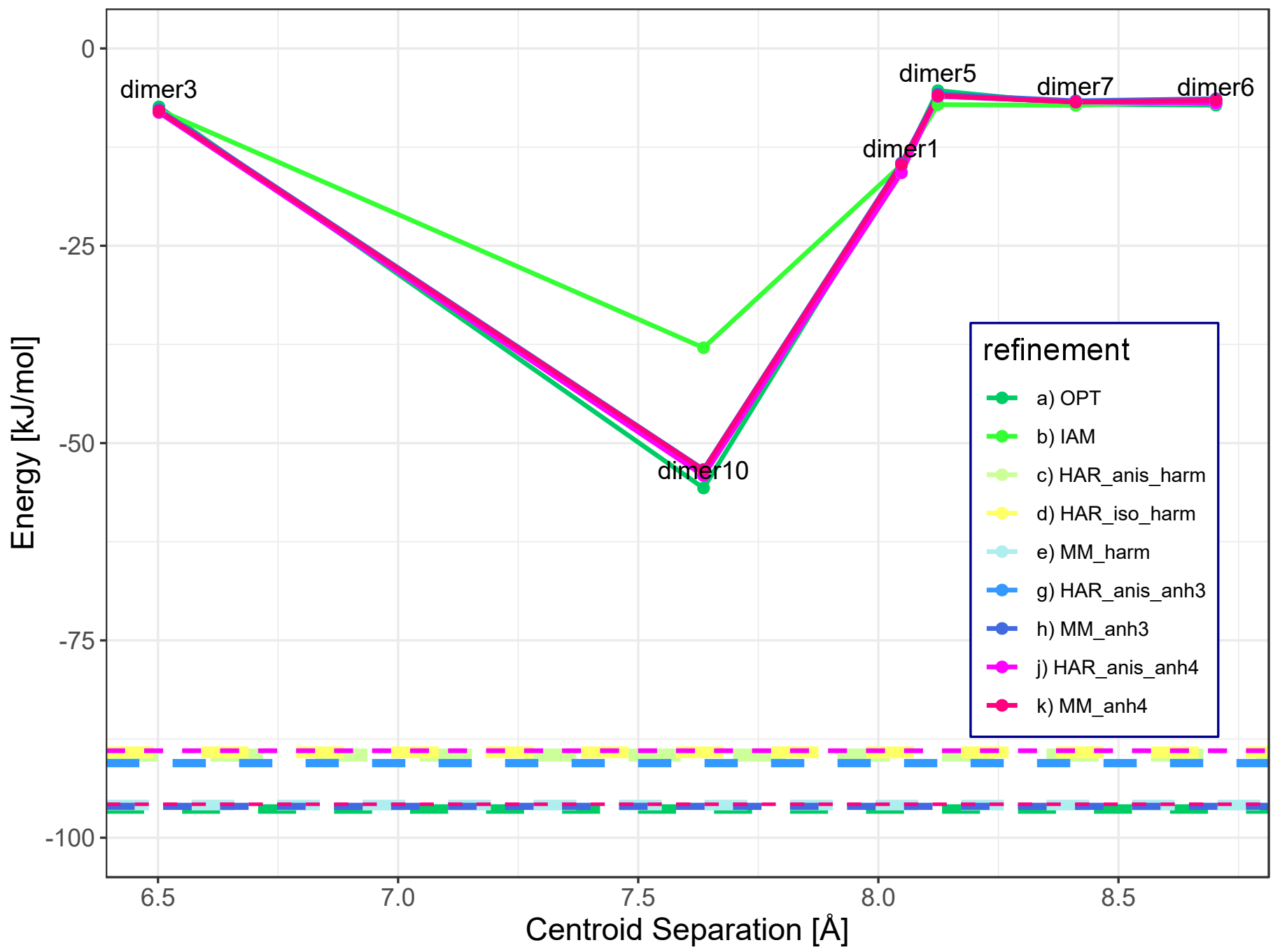

Figure 55: Dimer interaction energies (data points linked with solid lines) obtained for the structure of PT-8(Br) refined with intensity cut-off $(|F| \geq 2 \sigma(|F|))$ using various methods presented as a function of centroid separation of dimers. Cohesive energies are marked with dashed lines. 


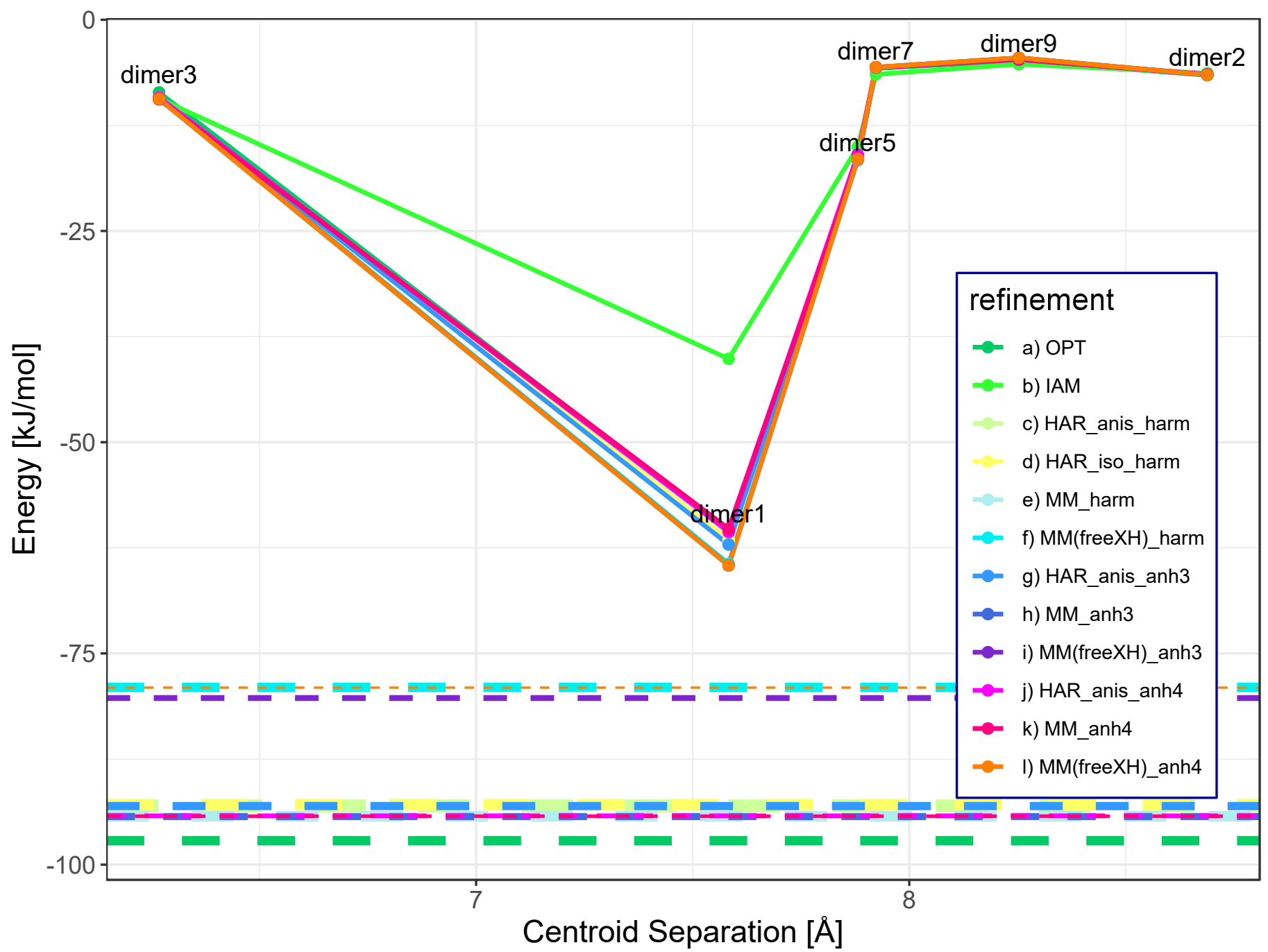

Figure 56: Dimer interaction energies (data points linked with solid lines) obtained for the structure of PT-10(I) refined with intensity cut-off $(|F| \geq 2 \sigma(|F|))$ using various methods presented as a function of centroid separation of dimers. Cohesive energies are marked with dashed lines. 


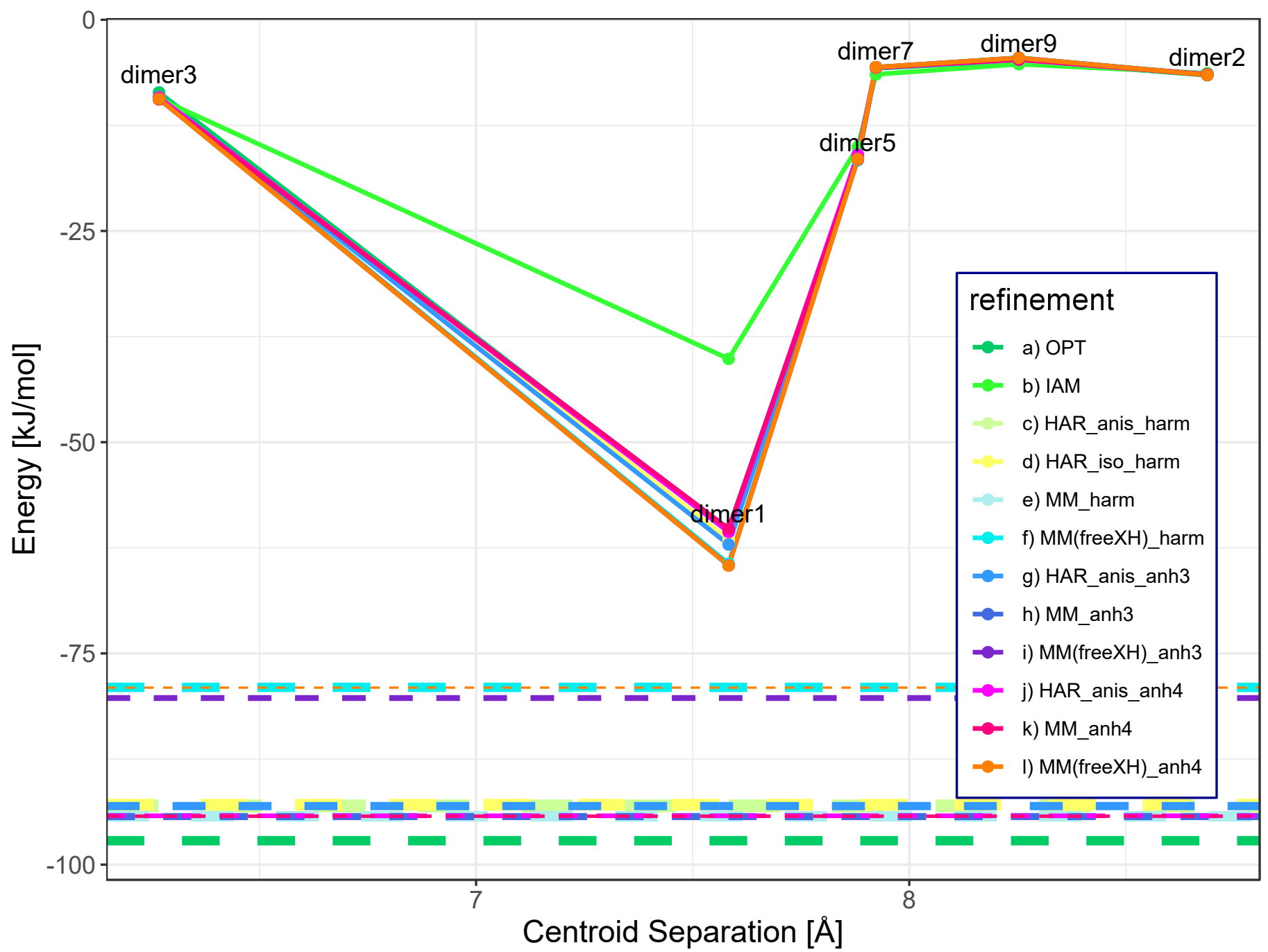

Figure 57: Dimer interaction energies (data points linked with solid lines) obtained for the structure of PT-11(S) refined with intensity cut-off $(|F| \geq 2 \sigma(|F|))$ using various methods presented as a function of centroid separation of dimers. Cohesive energies are marked with dashed lines. 


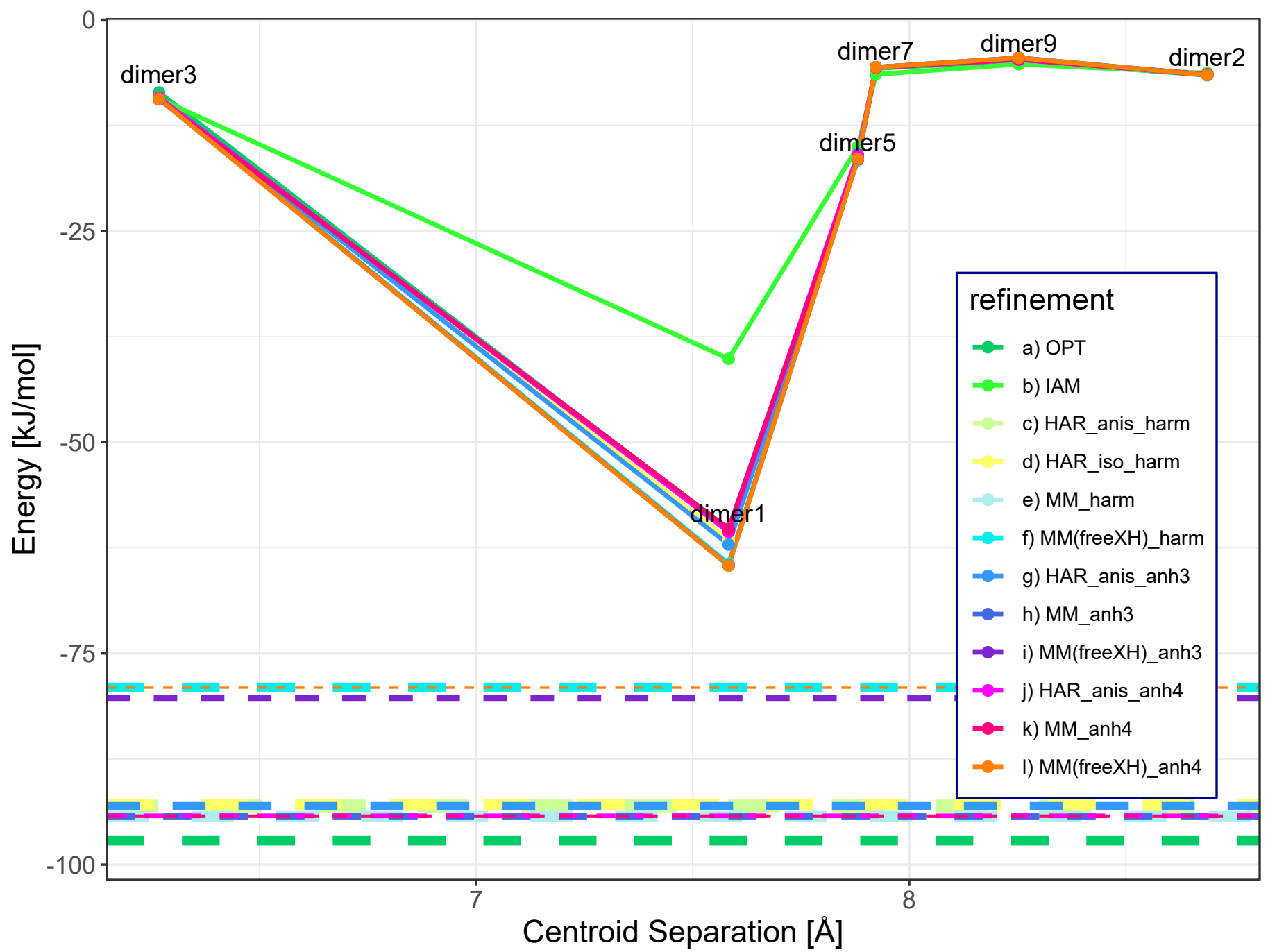

Figure 58: Dimer interaction energies (data points linked with solid lines) obtained for the structure of PT-2(Cl) refined without intensity cut-off using various methods presented as a function of centroid separation of dimers. Cohesive energies are marked with dashed lines. 


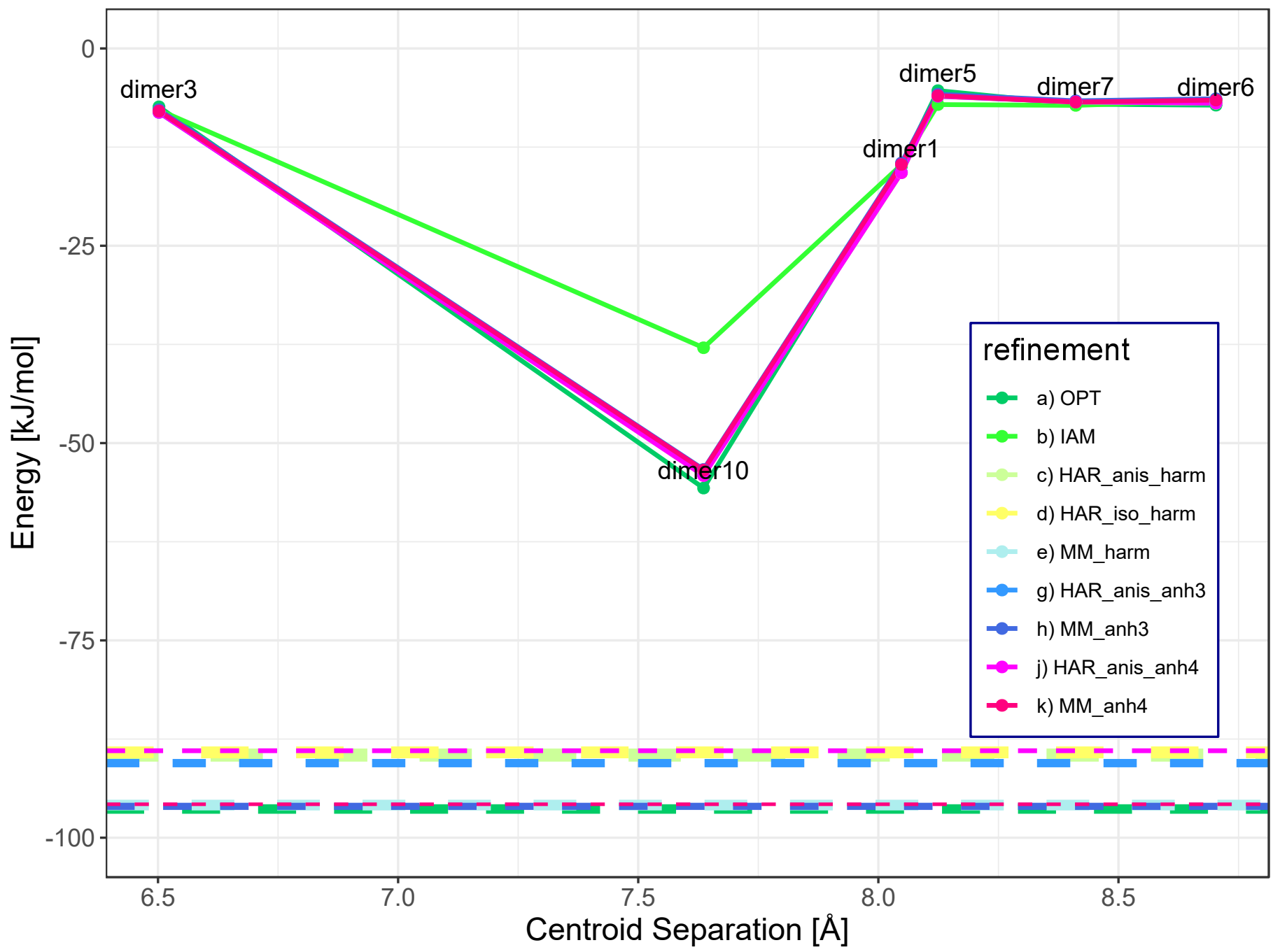

Figure 59: Dimer interaction energies (data points linked with solid lines) obtained for the structure of PT-8(Br) refined without intensity cut-off using various methods presented as a function of centroid separation of dimers. Cohesive energies are marked with dashed lines. 


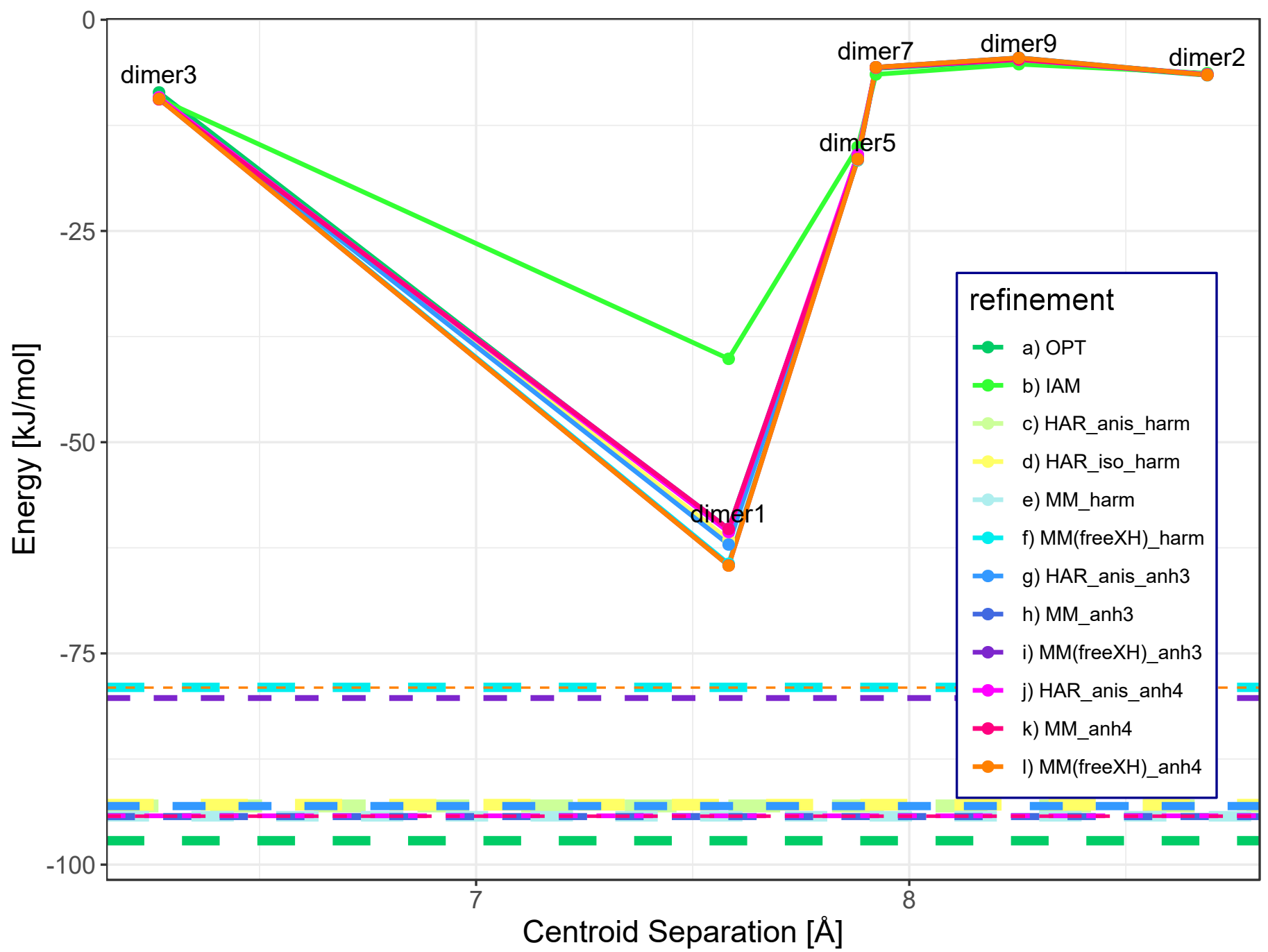

Figure 60: Dimer interaction energies (data points linked with solid lines) obtained for the structure of PT-10(I) refined without intensity cut-off using various methods presented as a function of centroid separation of dimers. Cohesive energies are marked with dashed lines. 


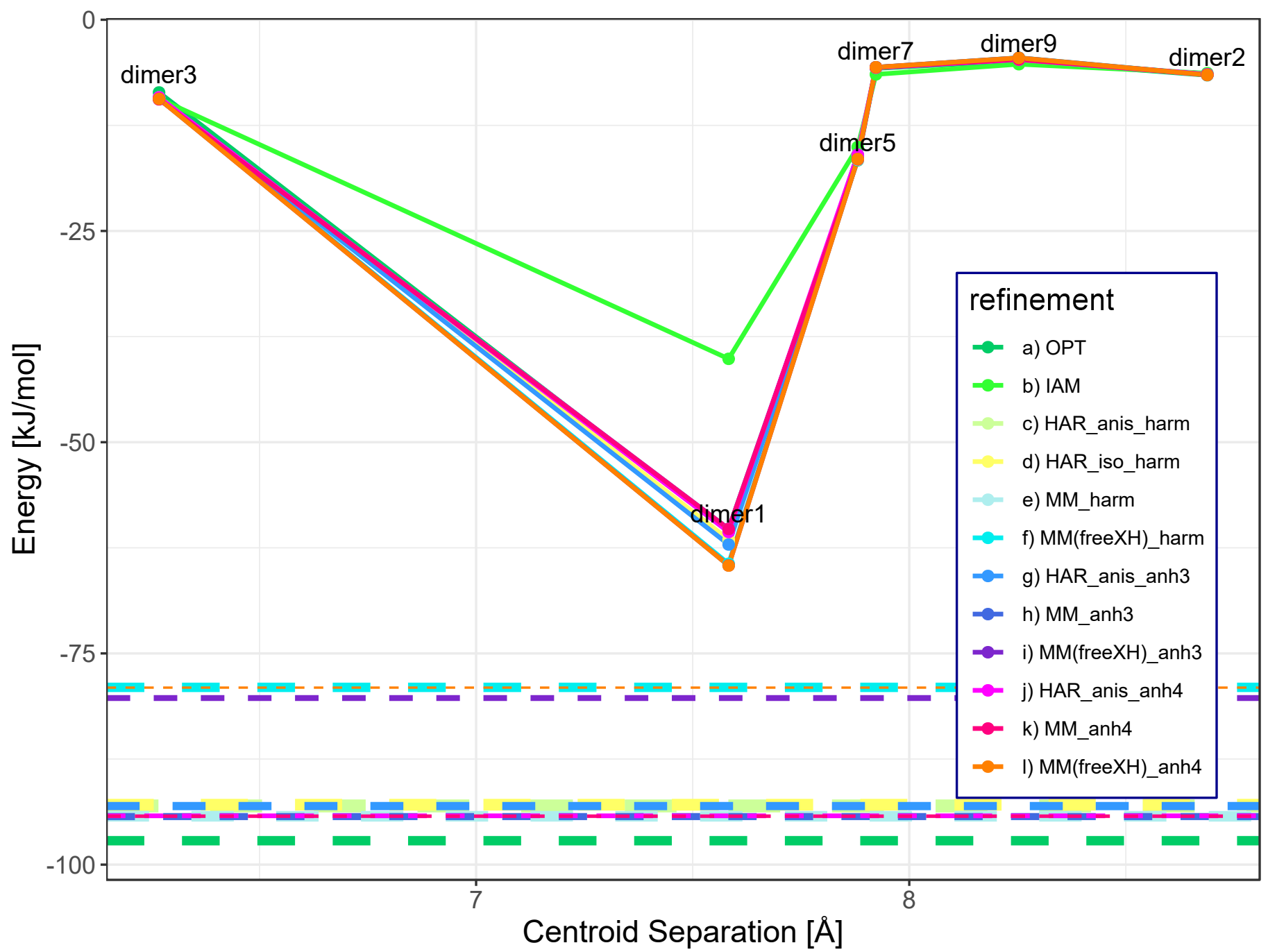

Figure 61: Dimer interaction energies (data points linked with solid lines) obtained for the structure of PT-11(S) refined without intensity cut-off using various methods presented as a function of centroid separation of dimers. Cohesive energies are marked with dashed lines. 


\section{Probability density function}
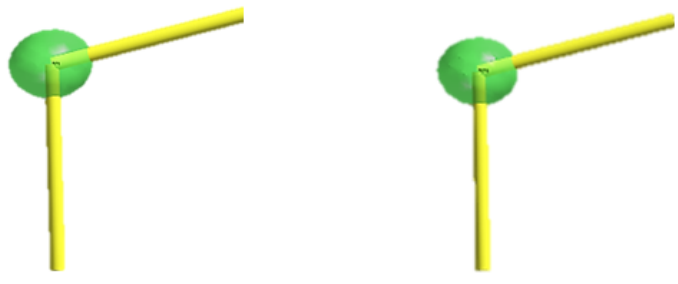

(a) HAR,

anharmonic $\mathrm{n}=3$, all reflections

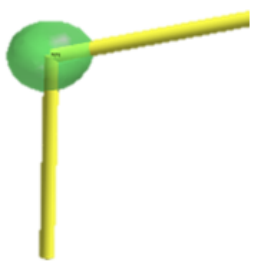

(e) MM,

anharmonic $\mathrm{n}=3$, all reflections

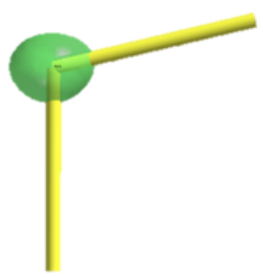
(i) $\mathrm{MM}($ freeXH) anharmonic $\mathrm{n}=3$, all reflections

(b) HAR,

anharmonic $\mathrm{n}=4$, all reflections

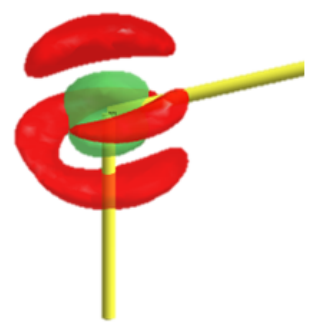

(f) MM,

anharmonic $\mathrm{n}=4$, all reflections

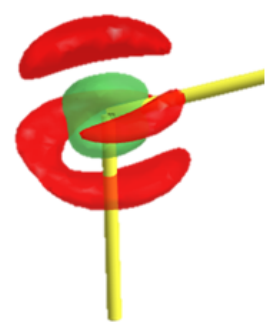

(j) $\mathrm{MM}($ freeXH) anharmonic $\mathrm{n}=4$ all reflections

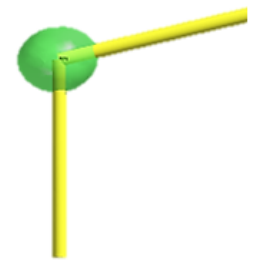

(c) HAR, anharmonic $\mathrm{n}=3$, $|F| \geq 2 \sigma(|F|)$

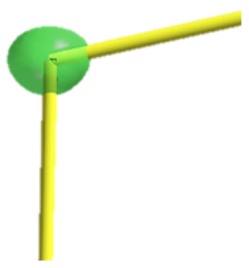

(g) MM, anharmonic $\mathrm{n}=3$, $|F| \geq 2 \sigma(|F|)$

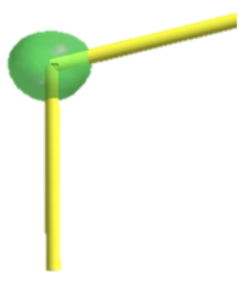

(k) $\mathrm{MM}($ freeXH), anharmonic $\mathrm{n}=3$, $|F| \geq 2 \sigma(|F|)$

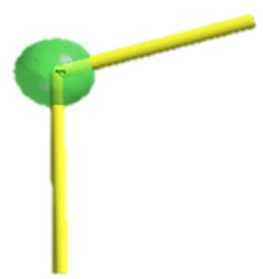

(d) HAR, anharmonic $\mathrm{n}=4$, $|F| \geq 2 \sigma(|F|)$

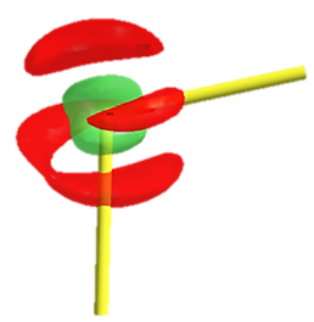

(h) MM, anharmonic $\mathrm{n}=4$, $|F| \geq 2 \sigma(|F|)$

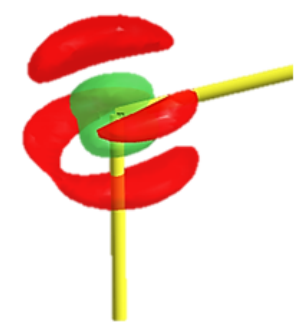

(l) $\mathrm{MM}($ freeXH), anharmonic $\mathrm{n}=4$, $|F| \geq 2 \sigma(|F|)$

Figure 62: Probability density function of the S atom in PT-11(S-Ph). Probability contour level: 50\%. Colours: green - positive, red - negative. 


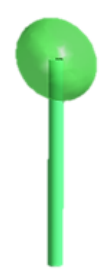

(a) HAR,

anharmonic $\mathrm{n}=3$, all reflections

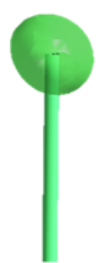

(e) MM,

anharmonic $\mathrm{n}=3$, all reflections

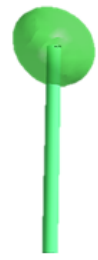

(i) $\mathrm{MM}($ freeXH), anharmonic $\mathrm{n}=3$, all reflections

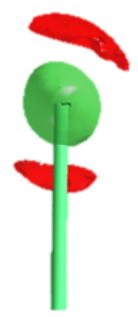

(b) HAR,

anharmonic $\mathrm{n}=4$, all reflections

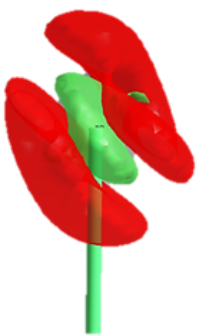

(f) MM,

anharmonic $\mathrm{n}=4$, all reflections

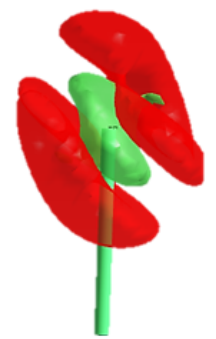

(j) $\mathrm{MM}($ freeXH), anharmonic $\mathrm{n}=4$, all reflections

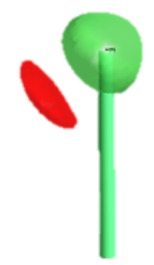

(c) HAR,

anharmonic $\mathrm{n}=3$, $|F| \geq 2 \sigma(|F|)$

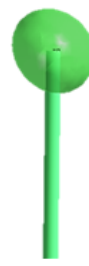

(g) MM,

anharmonic $\mathrm{n}=3$, $|F| \geq 2 \sigma(|F|)$

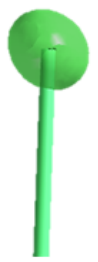

(k) $\mathrm{MM}($ freeXH), anharmonic $\mathrm{n}=3$, $|F| \geq 2 \sigma(|F|)$

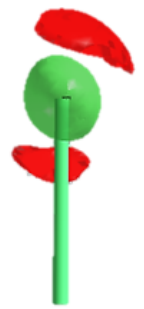

(d) HAR, anharmonic $\mathrm{n}=4$, $|F| \geq 2 \sigma(|F|)$

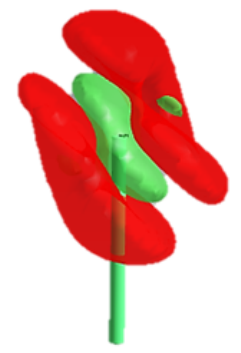

(h) MM, anharmonic $\mathrm{n}=4$, $|F| \geq 2 \sigma(|F|)$

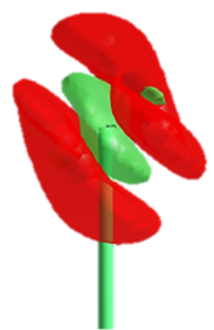

(l) $\mathrm{MM}($ freeXH), anharmonic $\mathrm{n}=4$, $|F| \geq 2 \sigma(|F|)$

Figure 63: Probability density function of the $\mathrm{Cl}$ atom in PT-2(Cl). Probability contour level: $50 \%$. Colours: green - positive, red - negative. 


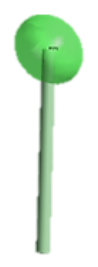

(a) HAR,

anharmonic $\mathrm{n}=3$, all reflections

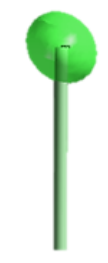

(e) $M M$,
anharmonic $n=3$,
all reflections

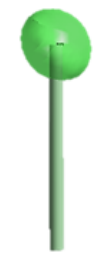

(b) HAR,

anharmonic $\mathrm{n}=4$, all reflections

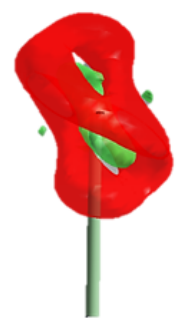

(f) MM, anharmonic $\mathrm{n}=4$, all reflections

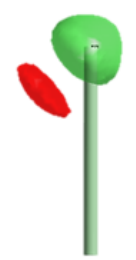

(c) HAR,

anharmonic $\mathrm{n}=3$, $|F| \geq 2 \sigma(|F|)$

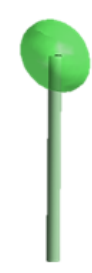

(g) MM,

anharmonic $\mathrm{n}=3$, $|F| \geq 2 \sigma(|F|)$ (d) HAR, anharmonic $\mathrm{n}=4$, $|F| \geq 2 \sigma(|F|)$

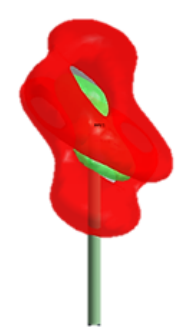

(h) MM, anharmonic $\mathrm{n}=4$, $|F| \geq 2 \sigma(|F|)$

Figure 64: Probability density function of the $\mathrm{Br}$ atom in PT-8(Br). Probability contour level: 50\%. Colours: green - positive, red - negative. 


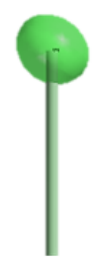

(a) HAR,

anharmonic $\mathrm{n}=3$, all reflections

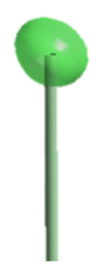

(e) MM, anharmonic $\mathrm{n}=3$, all reflections

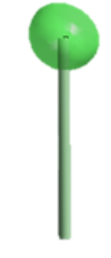

(b) HAR,

anharmonic $\mathrm{n}=4$, all reflections

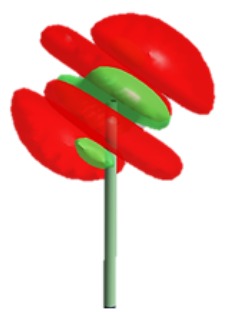

(f) MM, anharmonic $\mathrm{n}=4$, all reflections

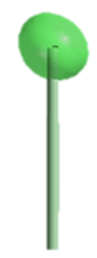

(c) HAR,

anharmonic $\mathrm{n}=3$, $|F| \geq 2 \sigma(|F|)$

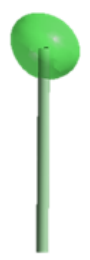

(g) MM,

anharmonic $\mathrm{n}=3$, $|F| \geq 2 \sigma(|F|)$ (d) HAR, anharmonic $\mathrm{n}=4$, $|F| \geq 2 \sigma(|F|)$

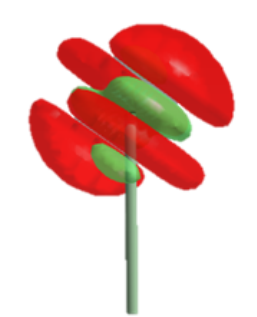

(h) MM, anharmonic $\mathrm{n}=4$, $|F| \geq 2 \sigma(|F|)$

Figure 65: Probability density function of the I atom in PT-10(I). Probability contour level: 50\%. Colours: green - positive, red - negative. 